\title{
Impact of stress exposure and pharmacological intervention on a mouse model of Alzheimer's disease
}

Citation for published version (APA):

Sierksma, S. R. (2012). Impact of stress exposure and pharmacological intervention on a mouse model of Alzheimer's disease. [Doctoral Thesis, Maastricht University]. Maastricht University. https://doi.org/10.26481/dis.20121220ss

Document status and date:

Published: 01/01/2012

DOI:

10.26481/dis.20121220ss

Document Version:

Publisher's PDF, also known as Version of record

\section{Please check the document version of this publication:}

- A submitted manuscript is the version of the article upon submission and before peer-review. There can be important differences between the submitted version and the official published version of record.

People interested in the research are advised to contact the author for the final version of the publication, or visit the DOI to the publisher's website.

- The final author version and the galley proof are versions of the publication after peer review.

- The final published version features the final layout of the paper including the volume, issue and page numbers.

Link to publication

\footnotetext{
General rights rights.

- You may freely distribute the URL identifying the publication in the public portal. please follow below link for the End User Agreement:

www.umlib.nl/taverne-license

Take down policy

If you believe that this document breaches copyright please contact us at:

repository@maastrichtuniversity.nl

providing details and we will investigate your claim.
}

Copyright and moral rights for the publications made accessible in the public portal are retained by the authors and/or other copyright owners and it is a condition of accessing publications that users recognise and abide by the legal requirements associated with these

- Users may download and print one copy of any publication from the public portal for the purpose of private study or research.

- You may not further distribute the material or use it for any profit-making activity or commercial gain

If the publication is distributed under the terms of Article $25 \mathrm{fa}$ of the Dutch Copyright Act, indicated by the "Taverne" license above, 


\section{Impact of stress exposure and pharmacological intervention on a mouse model of Alzheimer's disease}


Copyright (C) Susanne Rianneke Sierksma, Maastricht, 2012 ISBN: 978-94-6190-054-8

Impact of stress exposure and pharmacological intervention on a mouse model of Alzheimer's disease

Thesis with summary in English and Dutch

All rights reserved. No part of this thesis may be reproduced or transmitted in any form or by any means, electronic or mechanical, including photocopying, recording or by any information storage and retrieval system, without permission in writing from the author, or, when appropriate, from the publishers of the publications.

Cover design by Paperbrouwerij (Priscilla Brouwer)

Typesetting and layout: Annerieke Sierksma

Printed by Printservice Ede 


\title{
Impact of stress exposure and pharmacological
}

\author{
intervention on a mouse model of
}

\section{Alzheimer's disease}

\author{
Proefschrift \\ ter verkrijging van de graad van doctor \\ aan de Universiteit Maastricht, \\ op gezag van de Rector Magnificus, \\ Prof. Dr. L.L.G. Soete, \\ volgens het besluit van het College van Decanen, \\ in het openbaar te verdedigen op \\ donderdag 20 december 2012 om 14.00 uur \\ door
}

SusANNE RIANNEKE SiERKSMA

Geboren op 10 februari 1984 te Zwolle 


\section{Promotor:}

Prof. Dr. H.W.M. Steinbusch

\section{Copromotores:}

Dr. J. Prickaerts

Dr. D.L.A. van den Hove

\section{Beoordelingscommissie:}

Prof. Dr. H.H.H.W. Schmidt (voorzitter)

Prof. Dr. B. De Strooper (Katholieke Universiteit Leuven, België)

Dr. F.W. van Leeuwen

Prof. Dr. K.P. Lesch (University of Würzburg, Duitsland)

Prof. Dr. P.J. Lucassen (Universiteit van Amsterdam, Nederland)

Financial support for publication of this thesis was kindly provided by:
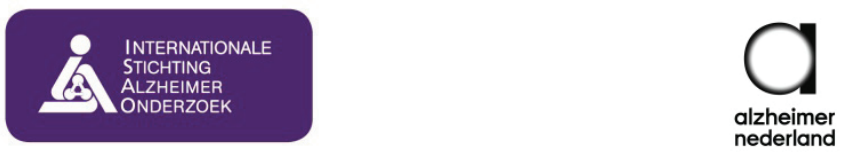

Internationale Stichting Alzheimer Onderzoek (ISAO) 


\section{"I am a brain, Watson. The rest of me is a mere appendix."}

Arthur Conan Doyle (1903) 'Sherlock Holmes-The Adventure of the Mazarin Stone' 

General Introduction

Chapter 2

Gene-environment interaction research and transgenic mouse models of

Alzheimer's disease

Chapter 3

Major depression, cognitive dysfunction and Alzheimer's disease: is there a link?

Chapter 4

Behavioral and neurobiological effects of prenatal stress exposure in male and female APPswe/PS1 $\triangle \mathrm{E} 9$ mice

Chapter 5

Effects of prenatal stress exposure on soluble $A \beta$ and brain-derived neurotrophic factor signaling in male and female APPSwe/PS1 $\triangle \mathrm{E} 9$ mice

Chapter 6

Chronic phosphodiesterase type 2 inhibition improves memory in the APPswe/PS1 $\triangle E 9$ mouse model of Alzheimer's disease

Chapter 7

Improvement of spatial memory dysfunction in APPswe/PS1 $\triangle \mathrm{E} 9$ mice after chronic inhibition of phosphodiesterase type 4D

Chapter 8

Increased mortality in APPswe/PS1 $1 \mathrm{E} 9$ mice after chronic fluoxetine treatment: a role for epileptic seizures?

$\begin{array}{rr}\text { Chapter } 9 & 195 \\ \text { General Discussion } & \\ \text { References } & \mathbf{2 0 3} \\ \text { Summary } & \mathbf{2 4 3} \\ \text { Vereenvoudigde samenvatting } & \mathbf{2 4 7} \\ \text { Appendices } & \mathbf{2 5 5} \\ \text { About the author } & 256 \\ \text { List of Publications } & 257 \\ \text { Affiliations of co-authors } & 260 \\ \text { Dankwoord } & \mathbf{2 6 5}\end{array}$





\section{Abbreviations}

$\begin{array}{ll}\text { 5mC } & \text { - 5'-methylcytidine } \\ \text { 5hmC } & \text { - 5'-hydroxymethylcytidine } \\ \text { 5-HT } & \text { - 5-hydroxytryptamine (serotonin) } \\ \text { A } 3 & \text { - amyloid- } \beta \\ \text { AC } & \text { - adenylate cyclase } \\ \text { AD } & \text { - Alzheimer's disease } \\ \text { ADAM } & \text { - a disintegrin and metalloproteinase } \\ \text { ANOVA } & \text { - analysis of variance } \\ \text { APP } & \text { - amyloid precursor protein } \\ \text { APOE } & \text { - apolipoprotein E } \\ \text { BACE1 } & \text { - } 3 \text {-amyloid cleaving enzyme 1 } \\ \text { BDNF } & \text { - brain-derived neurotrophic factor } \\ \text { BPSD } & \text { - behavioral and psychological symptoms of dementia } \\ \text { CAMP } & \text { - cyclic adenosine monophosphate } \\ \text { C99 } & \text { - C-terminal fragment of APP of 99 amino acids } \\ \text { CA1 } & \text { - cornu ammonis 1 of the hippocampus } \\ \text { CA3 } & \text { - cornu ammonis } 3 \text { of the hippocampus } \\ \text { CGMP } & \text { - cyclic guanosine monophosphate } \\ \text { CORT } & \text { - corticosterone } \\ \text { CREB } & \text { - cAMP response element-binding protein } \\ \text { CSF } & \text { - cerebral spinal fluid } \\ \text { d2 } & \text { - discrimination index of the object location task } \\ \text { DAB } & \text { - 3,3'-diaminobenzidine tetrahydrochloride } \\ \text { DCE } & \text { - differential contrast enhancement } \\ \text { DG } & \text { - dentate gyrus of the hippocampus } \\ \text { DNMT3a } & \text { - DNA methyltransferase 3a } \\ \text { E } & \text { - exploration time during a trial of the object location task } \\ \text { EZM } & \text { - elevated zero maze } \\ \text { FAD } & \text { - familial form of AD } \\ \text { FST } & \text { - forced swim test } \\ \text { GC } & \text { - guanylyl cyclase } \\ \text { GSK3 } 3 & \text { - glycogen synthase kinase 3 } \beta \\ \text { GWAS } & \text { - genome-wide association study } \\ \text { GxE } & \text { - gene-environment interaction } \\ \text { HPA axis } & \text { - hypothalamus-pituitary-adrenal axis } \\ & \end{array}$




\begin{tabular}{|c|c|}
\hline IR & - immunoreactivity \\
\hline IVC & - individually ventilated cage \\
\hline LOAD & - late-onset form of $A D$ \\
\hline LTP & - long-term potentiation \\
\hline OLT & - object location task \\
\hline OF & - open field test \\
\hline mBDNF & - mature BDNF \\
\hline $\mathrm{MCl}$ & - mild cognitive impairment \\
\hline MD & - major depression \\
\hline NA & - numerical aperture \\
\hline NO & - nitric oxide \\
\hline NOS & - nitric oxide synthase \\
\hline $\mathrm{p} 75^{\mathrm{NTR}}$ & - p75 neurotrophin receptor \\
\hline pCREB & - phosphorylated CREB \\
\hline PDE & - phosphodiesterase \\
\hline PDE-Is & - phosphodiesterase inhibitors \\
\hline PKA & - protein kinase $A$ \\
\hline PKG & - protein kinase $\mathrm{G}$ \\
\hline p.o. & - per os \\
\hline PS & - prenatal (maternal) stress \\
\hline PS1 & - presenilin 1 \\
\hline PS2 & - presenilin 2 \\
\hline RT & - room temperature \\
\hline sAPPa & - soluble APPa \\
\hline sAPP $\beta$ & - soluble APP $\beta$ \\
\hline s.c. & - subcutanous \\
\hline S.E.M. & - standard error of the mean \\
\hline SIPB & - synaptophysin-immunoreactive presynaptic bouton \\
\hline SIT & - sucrose intake test \\
\hline SSRI & - selective serotonin reuptake inhibitor \\
\hline $\mathrm{T}$ & - trial of the object location task \\
\hline TBS-T & - Tris buffered saline with $0.2 \%$ Triton X-100 \\
\hline TET1 & - Ten-eleven translocation 1 \\
\hline TrkB & - tropomyosin-related kinase B receptor \\
\hline WT & - wild-type \\
\hline
\end{tabular}




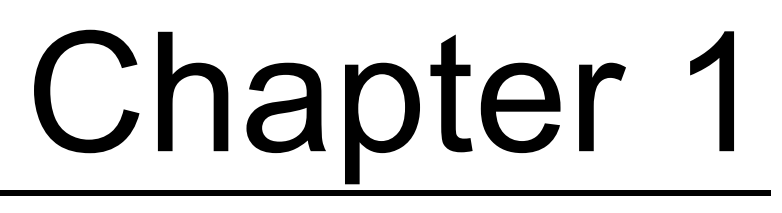

General Introduction 


\section{Alzheimer's disease - A historical overview}

At the $37^{\text {th }}$ Conference of South-West German Psychiatrists in Tübingen, Germany, in November 1906, Alois Alzheimer first presented the case of Auguste D., a 51-year-old woman suffering from a form of dementia later to be called Alzheimer's disease (AD). She had been admitted to the psychiatric institution in Frankfurt in 1901 by her husband, where she was examined by Alzheimer. According to Alzheimer, her clinical profile was peculiar and could not be categorized into one of the established illnesses recognized at that time [1]. Her symptoms included comprehension and memory impairments, aphasia (the inability to use or understand words), disorientation, paranoia, auditory hallucinations, and impairments in psychosocial behavior [1, 2].

Upon her death, 4.5 years later, Alzheimer examined Auguste D.'s brain. Considering this was a time when Freud's psychoanalysis was popular and psychiatric disorders were not commonly ascribed to physical disturbances, brain autopsy was not commonly performed. Yet, the founder of the psychiatric institution of Frankfurt, Heinrich Hoffmann, was a firm believer of biological origins of mental disorders, and so was Alzheimer's boss at that time, Emil Sioli [2, 3]. Given this stimulating environment and his close collaboration and friendship with Franz NissI (inventor of the 'Nissl staining'), Alzheimer was encouraged to further develop histological and microscopical examination of brain material $[3,4]$.

Examination of Auguste D.'s brain revealed gross atrophy and profound cell loss in several brain regions. Moreover, he identified the two hallmarks still valid today for classification of dementia of the Alzheimer type: neurofibrillary tangles and extracellular plaques. Changes in neurofibrils were visualized with the help of the Bielschowsky silver stain, which had been recently developed: 'Inside of a cell which appears to be quite normal, one or several fibrils can be distinguished by their unique thickness and capacity for impregnation' [1]. Furthermore, he described plaque pathology: 'Distributed all over the cortex, but especially numerous in the upper layers, there are minute miliary foci which are caused by the deposition of a special substance in the cortex. This substance can be observed without dye, but it is very refractory to dyeing' [1]. He published his findings in 1907 as 'Uber einen eigenartigen schweren Erkrankungsproze $\beta$ der Hirnrinde' (Concerning a unique disease process of the cerebral cortex). In 1910, his superior Emil Kraepelin refers to Alzheimer's findings in his influential textbook 'Psychiatrie' and introduces the diagnostic term 'Alzheimer's disease' [2, 4].

\section{Current societal impact}

Today, $A D$ is the most common form of dementia, comprising approximately $60-70 \%$ of the 35.6 million people currently suffering from dementia worldwide [5]. It has been estimated that the annual costs of dementia to society is 604 billion US dollars worldwide [5] and 160 billion euro for the European Union alone, which is approximately 22.000 euro of health care and societal costs per demented person per year [6]. These figures highlight the enormous 
socioeconomic impact of $A D$ and other dementias on our health care system and stress the need for further research.

\section{Symptoms and disease course}

A definitive diagnosis of $A D$ can still only be established at post mortem examination of the brain. Before that time, a probable diagnosis can be determined based on brain imaging techniques, cerebral spinal fluid punctures and neuropsychological assessment. According to the Diagnostic and Statistical Manual of Mental Disorders [7], AD is characterized by progressive disturbances in a multitude of cognitive domains, which are severe enough to impair social or occupational functioning. Most prominently, AD patients suffer from memory impairment, combined with one or more of the following: aphasia (inability to use or understand words), apraxia (inability to execute movements), agnosia (inability to recognize objects or people) or disturbances in executive functioning (i.e. planning and organizing). Besides the cognitive impairments, $50-90 \%$ of $A D$ patients also display behavioral and psychological symptoms of dementia (BPSD), such as aggression, agitation, anxiety, delusions apathy, and depression [8, 9].

From the time of diagnosis, survival of an $A D$ patient is on average 7 years [10]. Although the World Health Organization places $A D$ and other dementias on the $4^{\text {th }}$ place of leading causes of death in high-income countries in 2008 [11], it remains difficult to directly attribute mortality to dementia. Demented patients often display other physical disturbances, which may or may not be the consequence of the dementia process itself [5]. The most frequent causes of death reported for demented patients include bronchopneumonia (often caused by swallowing disorders), cardiovascular disease, malnutrition, dehydration and immobility [12-15].

\section{Neuropathological hallmarks}

As $A D$ is a neurodegenerative disorder, brain atrophy is one of its features, as observed by an enlargement of the sulci and ventricles $[16,17]$. This atrophy has been mainly attributed to loss of gray matter, i.e. brain cells and dendrites $[16,18]$. Neuron loss has shown to be highly region- and cell-specific, mainly encompassing the pyramidal cells in the entorhinal cortex, hippocampal formation and distinct (sub)cortical areas involved in learning and memory processes and higher cognitive processing [18-22]. Extensive synapse loss has also been reported in $A D$ patients $[23,24]$, and has shown to correlate very robustly with cognitive performance [25].

Other neuropathological hallmarks of $A D$ are the presence of 2 different types of protein aggregations: intracellular neurofibrillary tangles and extracellular plaques. Neurofibrillary tangles are comprised of the protein tau, which, under non-pathological conditions, stabilizes the axonal microtubuli that shape the cell's cytoskeleton. However, in AD, tau becomes hyperphosphorylated, which leads to a detachment from the microtubuli, and the 
hyperphosphorylated tau eventually becomes aggregated into neurofibrillary tangles, as often seen in the neurons of AD patients [26].

Extracellular plaques consist of the amyloid- $\beta(A \beta)$ protein. $A \beta$ is formed from proteolytic cleavage of the transmembrane amyloid precursor protein (APP). APP can be cleaved via two different pathways: a non-amyloidogenic and a amyloidogenic pathway (see Figure 1). The major pathway is the non-amyloidogenic pathway, which is driven by $\alpha$-secretase (e.g. a disintegrin and metalloproteinase (ADAM) 10 and 17), cleaving APP within the A $\beta$ sequence, thereby avoiding production of $A \beta$ and releasing the soluble APPa (sAPPa) into the extracellular space $[27,28]$. The alternative amyloidogenic pathway cleaves APP by means of $\beta$-secretase (also known as $\beta$-amyloid cleaving enzyme 1 (BACE1)), generating both soluble APP $\beta$ (sAPP $\beta$ ), which is secreted, and a membrane-tethered C-terminal fragment of 99 amino acids, C99. The $\mathrm{Y}$-secretase complex, comprised of the subunits anterior pharynxdefective 1, nicastrin, presenilin enhancer protein 2 and presenilin 1 (PS1) or 2 (PS2) [29], is responsible for the subsequent cleavage of C99. This cleavage will yield both the APP intracellular domain (AICD), which can translocate to the nucleus and modulate gene transcription, as well as the $A \beta$ peptide $[27,28]$. It is believed that $A \beta$ is formed by a first endoproteolytic cleavage of $\gamma$-secretase, generating longer $A \beta$ species (48-52 amino acids in length), and subsequent carboxypeptidase-like cleavages of $\gamma$-secretase yielding shorter forms of $A \beta$ (i.e. 38, 40 or 42 amino acids long) [30, 31], which can all be secreted. Due to their hydrophobic properties, $A \beta$ peptides are prone to aggregate in the extracellular space, first forming oligomers (e.g. dimers, trimers), then $A \beta$ fibrils and ultimately forming the characteristic plaques also described by Alois Alzheimer.

According to the amyloid cascade hypothesis [32], the deposition of $A \beta$, as a result of a disbalance between $A \beta$ production and clearance, is the initiating factor in the disease process. This event would trigger the formation of neurofibrillary tangles, induce synapse and neuron loss and would ultimately lead to dementia symptoms. It was first believed that the actual plaques induced the toxic effects, as neurons surrounding the plaques often show signs of neurodegeneration, such as dystrophic neurites [33]. However, as correlations between plaque burden and cognitive impairments could not be unequivocally established $[25,34]$, and also non-demented patients can display plaque pathology [35], the field shifted to pinpointing various soluble $A \beta$ oligomers and intraneuronal $A \beta$ as the driving force for pathology onset $[36,37]$. Although the existence of intraneuronal $A \beta$ has recently been questioned [38], and also the precise character of the toxic $A \beta$ oligomers needs further identification [39], it is still generally accepted that the formation of $A \beta$ plays a pivotal role in the etiology of $A D$. 


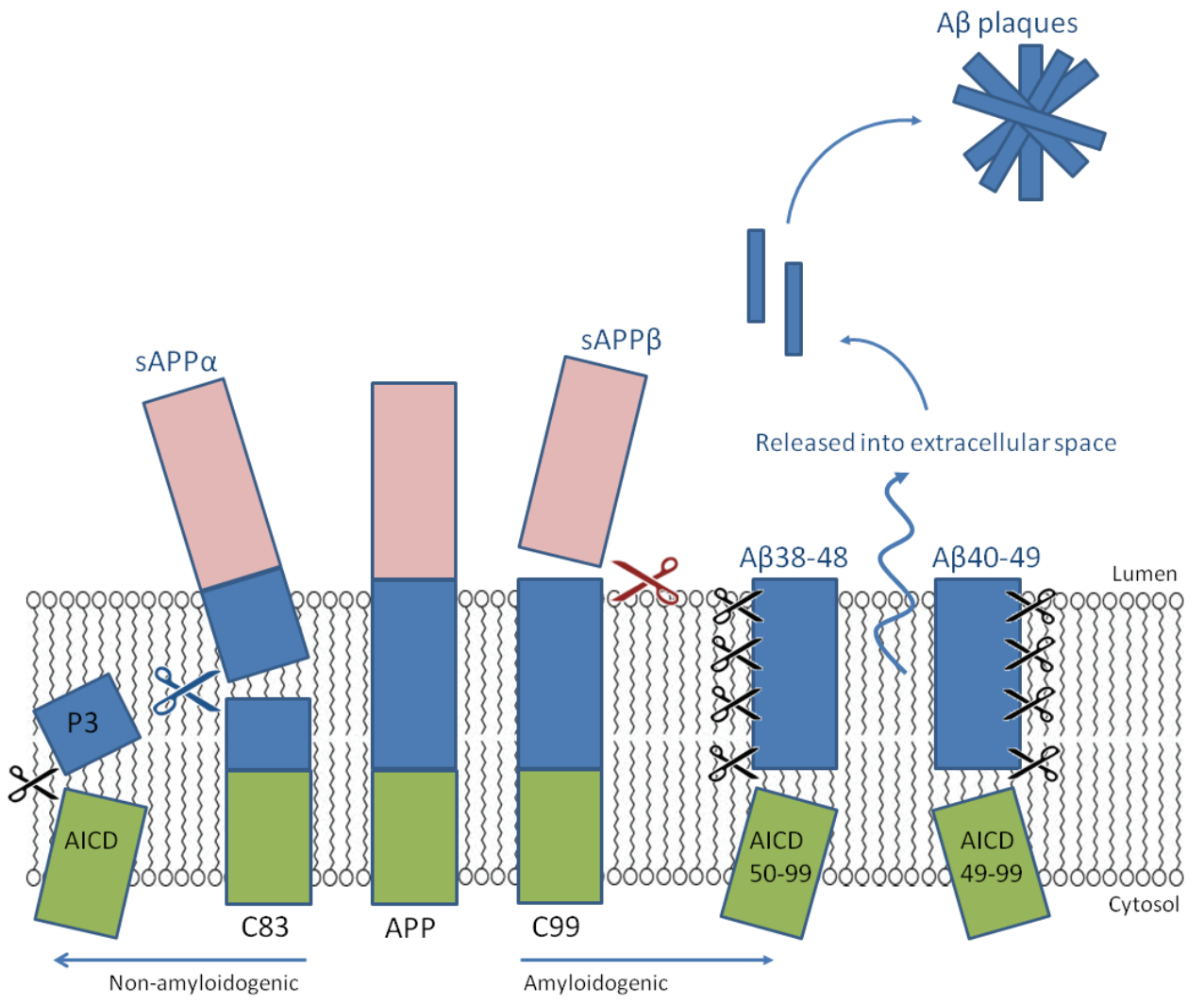

$\mathcal{K}=a$-secretase $\mathscr{K}=\beta$-secretase $\mathscr{K}=\gamma$-secretase

Figure 1: Schematic representation of the proteolytic cleavage of the transmembrane amyloid precursor protein (APP) via the non-amyloidogenic (left) and amyloidogenic pathway (right). Cleavage of APP via the non-amyloidogenic pathway entails a first proteolytic cleavage by $\alpha$-secretase within the $A \beta$ sequence (blue section), yielding a soluble APPa (sAPPa) and a C83 fragment. The C83 fragment can be further cleaved by $y$-secretase, producing the APP intracellular domain (AICD) and the P3 fragment. Alternatively, cleavage via the amyloidogenic pathway first requires proteolytic cleavage by $\beta$ secretase, releasing the soluble APP $\beta$ (SAPP $\beta$ ) fragment as well as retaining the C99 fragment within the membrane. Subsequent cleavage of $C 99$ by $\gamma$-secretase can yield one of two $A \beta$ product lines, i.e. an $A \beta 38 / 42$ or $A \beta 40 / 43$ line, depending on the first endoproteolytic cleavage of $\gamma$-secretase ([31]). After the first cleavage, a 48 or 49 amino acid long $A \beta$ fragment remains which can be cleaved further via carboxypeptidase-like cleavage of $A \beta$ by $\gamma$-secretase, generating $A \beta$ fragments of various length. Depending on their hydrophylic properties, these $A \beta$ peptides can be released into the extracellular space, where they will aggregate and deposit into amyloid plaques. 


\section{Forms of $A D$}

Based on genetic endowment, 2 forms of $A D$ can be distinguished: one that is inherited via an autosomal dominant pattern, i.e. familial $A D(F A D)$, and one that is not, i.e. sporadic $A D$. For the FAD form, 3 genes have been identified to date, namely the APP gene, located on chromosome 21, the PS1 gene, on chromosome 14, and the PS2 gene, on chromosome 1. As all three genes encode for proteins involved in $A \beta$ production, these genetic mutations form the strongest line of evidence in favor of the amyloid cascade hypothesis. Similarly, people with trisomy 21 (Down's syndrome), who naturally overexpress APP, also display plaque pathology from an early age onwards, and often develop dementia in their mid-forties $[40,41]$.

FAD mutations are rare and account for only approximately $5 \%$ of all $A D$ cases [42]. Whereas FAD patients often display an early disease onset, i.e. before the age of 65 , sporadic $A D$ cases mostly develop the disorder at an older age. Hence, age is main the risk factor for the sporadic cases [43], although genetic susceptibility genes have been identified as well. The main genetic risk factor for sporadic AD is the apolipoprotein E4 (APOE4) allele. Homozygous carriers of APOE4 have a 15-fold higher risk and heterozygous carriers a 3fold higher risk for $A D$ development compared to those without the APOE4 allele [44]. Powerful genome-wide association studies have further detected genes associated with $A D$, which may provide new insights into pathological pathways involved in $A D[45,46]$. As no clinical or neuropathological differences can be established between the sporadic and the $F A D$ cases, research on how the genetic mutations may explain the pathogenesis of $A D$ could provide insights into AD processes for the sporadic cases as well.

\section{Tools for AD research}

Studying the pathophysiology of $A D$ is a challenging endeavor. Whereas post mortem brain investigations have generated in depth knowledge on AD neuropathology, it is often limited to examining patients at the end of their disease course. And although epidemiological studies can give us more insight into risk factors and disease progression, the heterogeneity of the $A D$ population and unwanted influence of external factors can complicate interpretation of the data.

The discovery of genes implicated in the disease onset has led to the generation of multiple transgenic mouse models of $A D$, often over-expressing human $F A D$ mutations in APP, PS1 or PS2, sometimes in combination with overexpression of mutations in tau, which have been identified in frontal-temporal dementia patients (see Chapter 2 for an overview encompassing most mouse models of $A D$ ). The advantage of using mouse models is that both the genetic and environmental variables can be strictly controlled. Moreover, the disease process can be monitored during different phases of the disease, i.e. from before onset of pathology to advanced stages of the illness. 
In this dissertation, we have made use of the APPswe/PS1 $\triangle E 9$ mouse model of $A D$, created by Dr. David Borchelt and coworkers [47]. This mouse model carries a double genetic mutation: the Swedish mutation on the APP695 isoform (K694L/N595L), which was first discovered in a Swedish family, combined with the PS1 mutation which leads to a deletion of exon 9 within that gene. Both mutations are under the expression of the mouse prion protein promotor, thereby directing expression of both transgenes mainly to CNS neurons [48]. The human/mouse chimeric APP695 Swedish mutation leads to a 2-3 fold overexpression of the APP protein [48], while the PS1 $\triangle E 9$ mutation causes an increase in the production of $A \beta 42$ over $A \beta 40$ [49], thereby leading to plaque deposition from 4 months of age [50]. The first cognitive deficits are reported from 7 months of age onwards [51, 52]. The recapitulation of amyloid pathology and cognitive deficits as seen in $A D$ patients, and the relative quick onset of pathology and symptoms, makes the APPswe/PS1 $1 \mathrm{E} 9$ mouse model a suitable model for investigating AD-related processes.

\section{Current treatment options}

$A D$ is an incurable illness, and its treatment options are limited. Today, the only types of medication approved for the treatment of $A D$ are acetylcholinesterase inhibitors, e.g. rivastigmine and donepezil, and one NMDA-receptor antagonist, i.e. memantine [53]. Whereas acetylcholine esterase inhibitors prevent the enzymatic breakdown of the neurotransmitter acetylcholine in the synaptic cleft, thereby enhancing synaptic transmission, NMDA-receptor antagonists prevent the excitotoxic effects of glutamate on neurons, rendering them less prone to AD's neurodegenerative character. However, these current treatment options are limited in efficacy, often only aiding patients with moderate dementia. Moreover, gastrointestinal side effects, such as nausea, are frequent, and at times severe enough to cause cessation of treatment [54]. Given the increasing number of people suffering from $A D$, it is clear that more efficacious therapeutic agents are urgently needed.

\section{Phosphodiesterase inhibitors as potential therapeutic agents}

Possible new candidates for the treatment of $A D$ could be phosphodiesterase (PDE) inhibitors (PDE-Is). Acute and/or chronic administration of specific PDE-Is, in rodents, macaques and humans has shown to improve performance in various cognitive domains, including learning and memory processes, attention, cognitive flexibility, information processing, and executive functioning (for an extensive overview, see [55]), many processes of which are impaired in AD. PDE-ls prevent the enzymatic breakdown of the cyclic nucleotides cyclic adenosine monophosphate (cAMP) and/or cyclic guanosine monophosphate (cGMP) by inhibiting PDE enzymes. PDEs can be classified into 11 families (PDE1-11), yet most of these families contain more than 1 gene product (e.g. PDE4A-D). 
Alternative splicing of genes generates extensive heterogeneity, leading to more than 100 mRNA products that have been identified to date [56]. PDEs can be selective for hydrolysis of only cAMP, e.g. PDE4, only cGMP, e.g. PDE5, or can target both cyclic nucleotides, e.g. PDE2.

The second messenger cAMP is synthesized from adenosine triphosphate (ATP) by adenylate cylcase (AC) and can activate protein kinase A (PKA). PKA can phosphorylate cAMP response element-binding protein (CREB), thereby affecting transcription of genes related to synaptic plasticity, like brain-derived neurotrophic factor (BDNF) [57]. Conversely, cGMP is derived from guanosine triphosphate (GTP) by guanylyl cyclase (GC), which in turn is activated by nitric oxide (NO). The NO/cGMP pathway then activates protein kinase $G$ (PKG), which can also induce CREB phosphorylation [58], possibly via modulation of PKA levels (unpublished data from our group). Both the CAMP/PKA/CREB and the cGMP/PKG/CREB pathway are implicated in long-term potentiation (LTP); the proposed neurophysiological correlate of memory [58-60] (see Figure 2).

$A \beta$ can disrupt the formation of LTP by interfering both with the CAMP/PKA/CREB and the cGMP/PKG/CREB pathway. Indeed, $A \beta$ peptides were able to decrease PKA activity, possibly by preventing the degradation of PKA's regulatory subunits [61]. Moreover, $A \beta$ has shown to inhibit NO synthase activity, which produces $N O$, by impairing the recruitment of NADPH [62], but also to decrease GC activity [63, 64], thereby decreasing cGMP levels and PKG activation (see Figure $2 A$ ). It could be speculated that the detrimental effects of $A \beta$ on intracellular signaling could be counteracted by restoration of the levels of cyclic nucleotides with the help of PDE-Is (see Figure 2B).

The beneficial effects of PDE-Is on AD-related symptoms and neuropathology have indeed been demonstrated in mouse models of $A D$. Both acute and chronic treatment with the PDE4-I rolipram induced memory improvement and a restoration of LTP deficits [65, 66]. Comparable results were reported for chronic treatment with the PDE5-I vardenafil, yet this compound could also reduce hippocampal $A \beta$ levels [67]. However, as isoform aspecific PDE4-Is, such as rolipram, can induce substantial emetic side effects [68], and the level of PDE5 expression in the brain is drastically decreased in aged individuals [69], both of the tested compounds will not be suitable for use in $A D$ patients. It is clear that fine-tuning of PDE-I compounds for the treatment of $A D$ is necessary. Perhaps, dual substrate or isoformspecific PDE-ls are good candidates for future research on suitable and effective $A D$ treatment. 


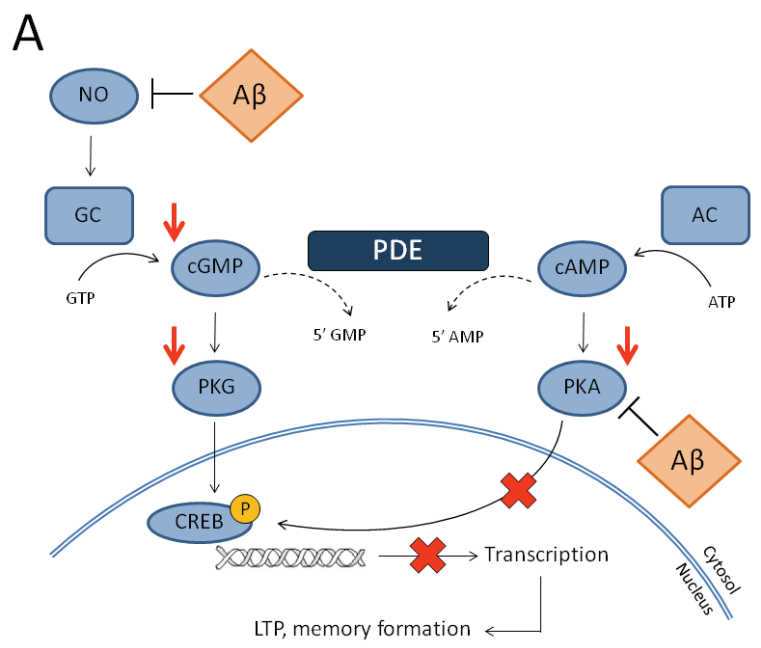

B

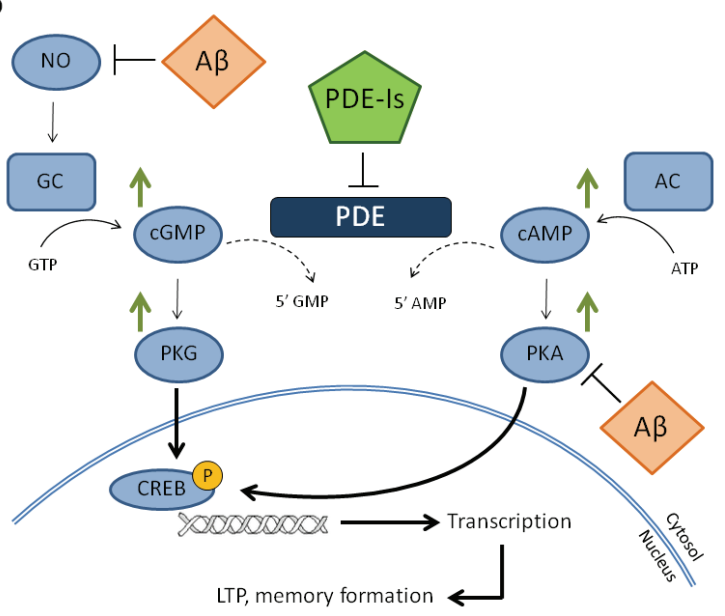

Figure 2: Schematic representation of the effects of amyloid- $\beta$ ( $A \beta$; in $A$ ) and phosphodiesterase (PDE) inhibitors (PDE-ls; in B) on intracellular signaling pathways. A: The cyclic nucleotide cyclic guanosine monophospate (cGMP) is synthesized from guanosine triphosphate (GTP) by guanylyl cyclase (GC), which in turn is activated by nitric oxide (NO). This NO/cGMP pathway then activates protein kinase $G$ (PKG), which leads to phosphorylation (p) of cAMP response element-binding protein (CREB), by modulating protein kinase A (PKA) activity. Similarly, cylcic adenosine monophosphate (cAMP) is derived from adenosine triphosphate (ATP) by adenylyl cyclase (AC), which can activate PKA, leading to phosphorylation of CREB, and subsequent transcription of genes involved in long-term potentiation (LTP) and memory formation. A $\beta$ can inhibit the NO/cGMP pathway as well as PKA activation, thereby interfering with CREB phosphorylation and memory formation. B: The intracellular levels of CAMP and cGMP are tightly regulated via hydrolysis of the cyclic nucleotides by PDEs. PDE-Is can prevent this enzymatic breakdown, thereby elevating the levels of cyclic nucleotides, and activating PKG and PKA. This can lead to a re-instatement of LTP and could enhance learning and memory processes. Based on $[61,62,70]$. 


\section{Gene-environment interactions}

A complicating factor in the treatment and prevention of $A D$ is the fact that its etiology remains largely unknown. Merely $5 \%$ of patients carry the FAD mutations, leaving $95 \%$ of $A D$ patients with an unidentified etiopathogenesis. Although certain genetic risk factors have been identified, it is clear that personal variability in genetic penetrance contributes to the expression of or relative protection from these risk genes. Evidently, if genetic factors cannot solely account for disease onset, other factors must contribute to AD etiology.

It has been shown that certain environmental factors independently alter the risk of developing $A D$. For example, exposure to metals or traumatic brain injury has shown to initiate disease onset, whereas Mediterranean diet and physical exercise can delay $A D$ development (see Chapter 2 for an extensive review). Of particular interest have been stress and stress-related disorders as risk factor for $A D$. Both the personality trait distress proneness, as well as a history of major depression can increase the likelihood of $A D$ development by at least twofold [71-74] and episodes of major depression can accelerate disease onset in FAD cases [75]. Moreover, AD patients with a lifetime history of major depression have a more rapid cognitive decline and higher expression of plaques and tangles in the hippocampus, than patients without such history. Similarly, chronic stress exposure in mouse models of $A D$ can induce earlier manifestation of $A D$-related cognitive deficits and increased plaque deposition, soluble $A \beta$ levels, tau phosphorylation and neurodegeneration [76-78].

Although both genes and environment can independently alter the risk for $A D$ development, other fields of neuropsychiatric research have shown that research on geneenvironment interaction (GxE) can yield novel insights into disease etiopathogenesis [79]. The GxE concept postulates that although genetic and environmental factors can have an independent impact on disease initiation, in some instances only the synergistic contribution of both genetic susceptibility and environmental exposure will lead to the induction of a certain phenotype, in this case $A D$ manifestation. It must be noted that this concept embodies both the notion of the environment being able to alter genetic susceptibility, but also that certain risk genes can alter how the environment is perceived and whether or not it will affect an individual. 


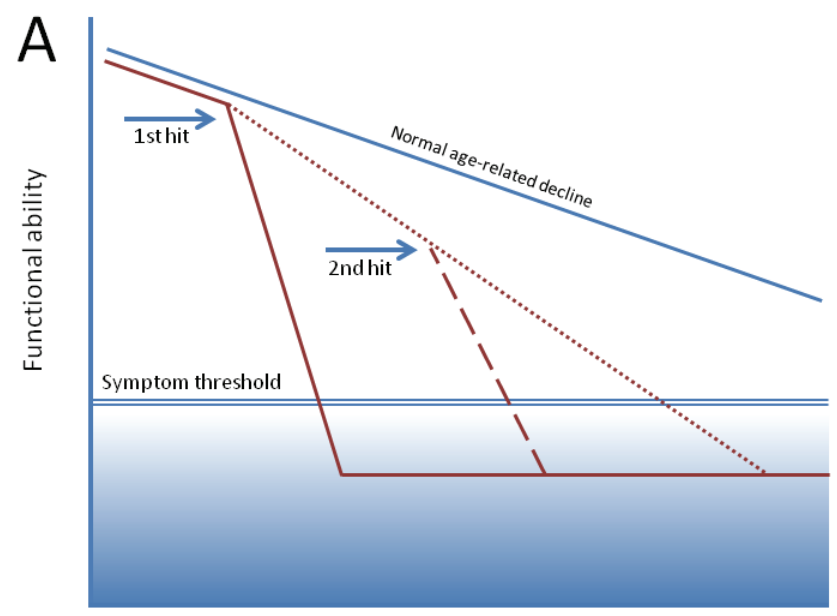

Age (years)

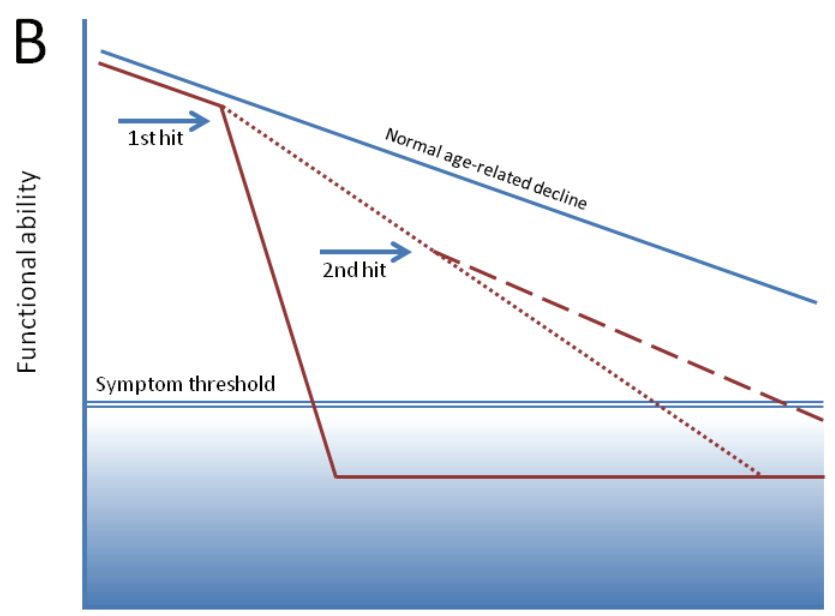

Age (years)

Figure 3: Schematic representation of the Latent Early-life Associated Regulation (LEARn) theory by Lahiri and colleagues. This model postulates that many neurobiological disorders, among which sporadic Alzheimer's disease (AD), are characterized by a mismatch between disease initiating events (i.e. 'hits') and manifestation of symptoms. A: In certain instances, a single hit can swiftly trigger the onset of symptoms which persist over time (continuous dark red line). In other instances, a first hit, e.g. a susceptibility gene or environmental exposure, can induce a diseased state but only after considerable time has passed, as may be the case for sporadic AD (finely dotted dark red line). Alternatively, the two-hit latency model proposes the occurrence of a first hit that by itself may or may not be sufficient to induce a diseased state. Yet, if the first hit is followed by subsequent hits later in life, this will indefinitely lead to manifestation of the disease (interspaced dark red). B: Along the same line, we extend the model, proposing that certain second hits can also have a protective function against disease development, and may therefore postpone or even prevent the onset of symptoms. Adapted from [80]. 
The GxE concept is also an integral part of the Latent Early-life Associated Regulation (LEARn) model by Lahiri and coworkers [80], which was formulated in response to their research on postnatal lead exposure and induction of genetic alterations later in life, leading to aberrant expression of $A \beta$-related genes [81, 82]. Within their model, diseases were categorized based on their progression, i.e. acute, chronic and latent disorders. Of particular interest to the AD field are the 'one-hit' and 'two-hit' latency models, which propose that certain events during development can prime genes to altered expression, yet this differential expression may have a delay in its manifestation (see Figure 3). Whereas in the 'one-hit' model only one risk gene or developmental disturbance may be enough to trigger disease onset later in life, the 'two-hit' model poses that only those subjects having had the first hit will be susceptible for the subsequent hit(s) to induce disease initiation. To extend this model further, it could also be argued that if one of these events would be neuroprotective, it may render the individual (partly) resilient against disease induction (see Figure 3B).

\section{Aim and outline of this thesis}

The research presented in this thesis was conducted within the GxE concept. The general aim was to investigate how changes in the environment can affect the development and progression of $A D$. In particular, we wished to explore how exogenous factors, such as chronic stress exposure and pharmacological agents, would impact on disease manifestation. To this extent, the APPswe/PS1 $\triangle E 9$ mouse model of $A D$ was used to investigate alterations in its $A D$ phenotype in terms of behavior and neuropathology (see Figure 4).

First of all, an overview of the literature is provided in Chapter 2, describing the impact of environmental exposures on the risk of $A D$ development and the progression of $A D$-related symptoms and neuropathology. Epidemiological reports on environmental exposure will be outlined, but also how these environmental exposures have been modeled in various mouse models of $A D$. It is further explored how knowledge on $G x E$ in $A D$ research can advance the field in understanding the full complexity of $A D$ etiopathogenesis.

The remaining content of this dissertation is subdivided into 2 parts, each dealing with a different environmental exposure. 


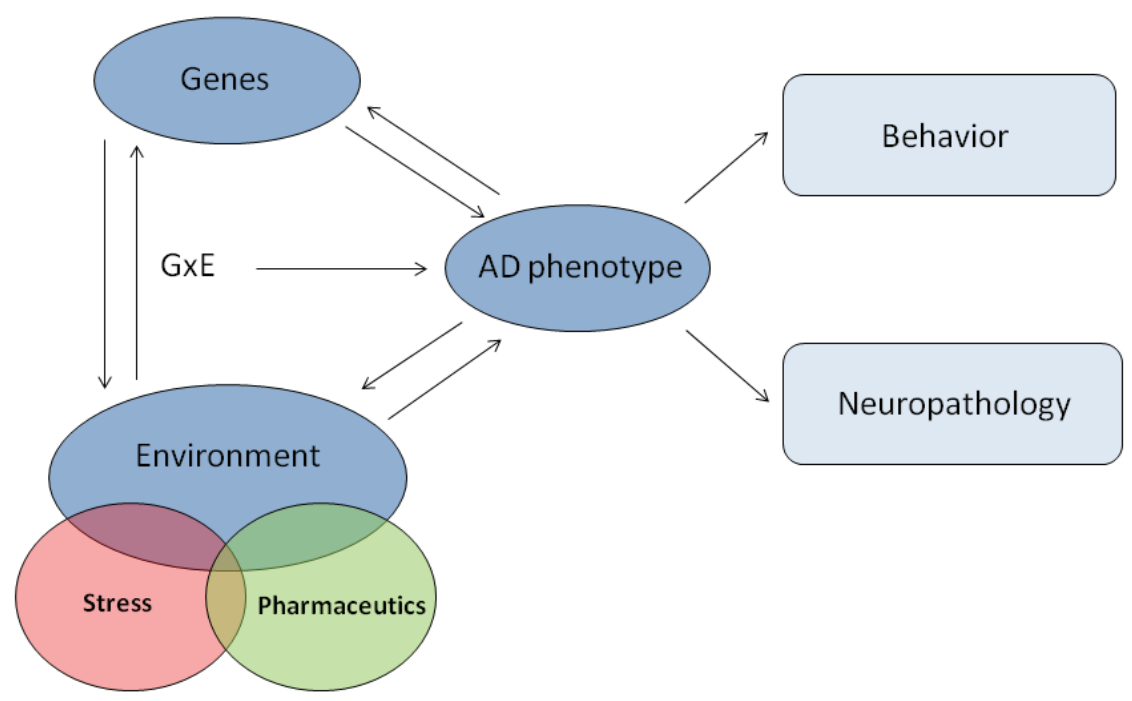

Figure 4: Schematic representation of the framework of this thesis. The expression of an Alzheimer's disease (AD) phenotype can be influenced by genes and the environment separately, but also by the synergistic interaction of genes and environment (GxE). The aim of this thesis was how alterations in the environment, either exposure to stress or pharmaceutics, could alter the expression of the $A D$ phenotype. By using the APPswe/PS1 $\triangle E 9$ mouse model of AD, both behavioral and neuropathological readout parameters were used to explore the extent of phenotypical alterations.

Part 1 of this dissertation will encompass the effects of stress exposure on the development and progress of AD-related symptoms and neuropathology. Chapter 3 summarizes the literature on stress exposure, major depression, cognitive decline and AD. In particular, it will discuss the role of the hypothalamic-pituitary-adrenal (HPA) axis and the serotonergic system in linking both major depression and $A D$. Based on the epidemiological findings stating that stress exposure serves as an independent risk factor of $A D$, it was explored how prenatal stress exposure, serving as a developmental model for major depression, could affect phenotypical expression of $A D$ in both male and female APPswe/PS1 $\triangle E 9$ mice. Chapter 4 discusses the alteration in affective, cognitive, neuroendocrine and neuropathological phenotype after prenatal stress exposure, while investigating the role of global epigenetic markers in regulating these phenotypical changes. Chapter $\mathbf{5}$ examines how prenatal stress exposure affects BDNF signaling and soluble $A \beta$ levels to further unravel the mechanism underlying the behavioral changes as described in Chapter 4 .

Part 2 of this dissertation explores the effects of pharmacological intervention on progression of AD-related behavior and brain function by using PDE-Is and the selective serotonin reuptake inhibitor (SSRI) fluoxetine. In Chapter 6 the efficacy of the dual substrate PDE2-I BAY60-7550 is investigated in view of preventing AD-related cognitive deficits. 
Additionally, underlying biochemical mechanisms such as CREB phosphorylation, BDNF signaling and $A \beta$ neuropathology are studied. Chapter 7 describes the effects of the novel PDE4D-I GEBR-7b in restoring AD-related memory impairments and examines whether treatment alters AD-related neuropathology, BDNF signaling and synaptic plasticity. In Chapter 8 APPswe/PS1 $1 \mathrm{E} 9$ mice were chronically treated with the selective serotonin reuptake inhibitor fluoxetine, to investigate whether antidepressant treatment could delay the cognitive deficits and onset of neuropathology observed in these mice.

Finally, the main results and overall conclusions are summarized and discussed in more detail in Chapter 9, while also providing possible future perspectives. 


\section{Chapter 2}

Gene-environment interaction research and transgenic mouse models of Alzheimer's disease

Annerieke S.R. Sierksma*, Leonidas Chouliaras*, Gunter Kenis, Jos Prickaerts, Marijke AM Lemmens, Ivona Brasnjevic, Eva L van Donkelaar, Pilar Martinez-Martinez, Mario Losen, Marc H De Baets, Natalia Kholod, Fred W van Leeuwen, Patrick R Hof, Jim van Os, Harry WM Steinbusch, Daniel LA van den Hove, Bart PF Rutten

*Joint first authors

International Journal of Alzheimer's disease (2010) Epub ahead of print 


\section{Abstract}

The etiology of the sporadic form of Alzheimer's disease (AD) remains largely unknown. Recent evidence has suggested that gene-environment interactions (GxE) may play a crucial role in its development and progression. Whereas various susceptibility loci have been identified, like the apolipoprotein E4 allele, these cannot fully explain the increasing prevalence of $A D$ observed with aging. In addition to such genetic risk factors, various environmental factors have been proposed to alter the risk of developing $A D$ as well as to affect the rate of cognitive decline in $A D$ patients. Nevertheless, aside from the independent effects of genetic and environmental risk factors, their synergistic participation in increasing the risk of developing $A D$ has been sparsely investigated, even though evidence points towards such a direction.

Advances in the genetic manipulation of mice, modeling various aspects of the $A D$ pathology, have provided an excellent tool to dissect the effects of genes, environment and their interactions. In this review we present several environmental factors implicated in the etiology of $A D$ that have been tested in transgenic animal models of the disease. The focus lies on the concept of GxE and its importance in a multifactorial disease like $A D$. Additionally, possible mediating mechanisms and future challenges are discussed. 


\section{Introduction}

Alzheimer's disease (AD) is the most common form of dementia, characterized by an initial loss of short-term memory, followed by a progressive impairment in multiple cognitive domains. The estimated lifetime risk for developing $A D$ is about $20 \%$ for women and $10 \%$ for men aged above 65 [83]. The pathology of $A D$ is characterized by an accumulation of misfolded proteins, oxidative damage and inflammatory changes ultimately resulting in region-specific loss of synaptic contacts and neuronal cell death [84]. Current biological theories on the etiology and pathology of $A D$ posit central roles for age-related molecular and cellular aberrations that induce an imbalance in the production, cleavage and clearance of amyloid- $\beta(A \beta)$, hyperphosphorylation of the tau protein, and aberrant apolipoprotein $E$ (APOE) function in the aging brain [83] Several genetic risk factors have been linked with an increased risk of developing $A D$, such as mutations in the amyloid precursor protein (APP) and presenilin (PS) 1 and 2 for the familial cases of AD (FAD), as well as the APOE4 allele for the sporadic late-onset form of $A D$ (LOAD). Several new genetic findings derived from powerful genome-wide association studies (GWAS; see below) have confirmed that $A D$ is a polygenic disorder. The genes identified in these studies may enlighten unknown biological pathways involved in AD [46].

Furthermore, various environmental exposures have been found to modify the risk of $A D$, such as diet and nutrition, physical exercise, exposure to metals, and brain trauma. Comorbidities such as vascular disorders or depression could also be of considerable importance, since these have also been suggested to contribute to the risk of AD. Recent evidence indicates that more attention should be paid to the role of the environment and its interactions with underlying genetic susceptibility, in triggering disease-related phenotypes [85]. The gene-environment interaction (GxE) approach differs from the linear approach of either genetic or environmental effects by positing a causal role not only for either genes or environmental exposures in isolation, but for their synergistic participation in leading to a certain phenotype (here AD) where the effect of one is conditional for the other [79, 86, 87]. Where epidemiological studies on $A D$ may reveal statistical evidence for GxE in the onset and course of $A D$, animal research can be instrumental to study the underlying biological mechanisms.

\subsection{Objective}

The objective of this review is to give an overview of the available transgenic mouse studies on $A D$, specifically addressing the concept of GxE. We start with a brief description of the various genetic and environmental risk factors of $A D$, and the different available transgenic mouse models of $A D$. The main part of the review describes the effects of several environmental exposures on AD-related phenotypes. These sections begin with a brief description of the epidemiological evidence in $A D$ (when available from meta-analyses), and continue with describing the findings from experimental animal studies in which the environmental factor was manipulated in $A D$ transgenic mice and, when performed, in wild- 
type (WT) mice. Thereafter, we discuss the strengths and limitations of these studies, and we end with identifying future challenges and prospects.

\section{Alzheimer's disease}

\subsection{Genetics of $A D$}

Twin studies on $A D$ have shown a heritability of $60 \%-80 \%$ and a concordance of $18 \%$ up to $83 \%$, depending on e.g. the population and age of the subjects investigated. Thus, both heritable and non-heritable factors play an important role in AD's age of onset, risk and etiology [88-91]. Several genetic risk factors have been linked to AD. Mutations in APP, PS1 and PS2 genes have consistently been associated with early-onset FAD. Also for LOAD several susceptibility loci have been linked with risk for $A D$, such as the gene encoding for the APOE4 allele or loci in the clusterin (CLU), phosphatidylinositol binding clathrin assembly protein (PICALM), complement receptor 1 (CR1), BIN1 (bridging integrator, amphiphysin) genes, a locus near the BLOC1S3 (biogenesis of lysosomal organelles complex1, subunit 3), and MARK4 (microtubule affinity-regulating kinase 4) genes [45, 46, $92,93]$. Other susceptibility loci have also been associated with $\operatorname{AD}[45,94]$

\subsection{Environment and $A D$}

Although a range of environmental exposures have been linked to $A D$, well-replicated and meta-analyses' evidence for the involvement of clear environmental factors in AD is sparse. Recent studies, however, have shown that dietary factors, such as exposure to a Mediterranean diet, fish and high omega-3 diets, cigarette smoking, head trauma, infections, systemic inflammation, and metal and pesticide exposure can significantly alter an individual's risk of developing AD. In addition, psychosocial factors such as education, social network, leisure activities and physical activity, chronic stress and depression, also seem to be either important protective or risk factors for AD [95-97]. Somatic factors that are under the direct influence of environmental exposures, such as blood pressure, obesity, diabetes mellitus, cardio- and cerebrovascular diseases and hyperlipidemia, have additionally been implicated in AD etiology [95, 97].

\subsection{Gene-environment interactions and $A D$}

The field of GxE research appears very promising for psychiatry and neuroscience, albeit still little investigated in $A D$ [98]. The notion of potential existence of $G x E$ in $A D$ has substantial impact on the interpretation of reports on genetic and non-genetic contribution to this disorder. Reported contributions of environmental and genetic factors to disease risk can be misleading, since they represent the environmental exposure in relationship with the genetic susceptibility or resilience to it [87]. Thus, the advantage of the concept of GxE is that it includes the genetic control of sensitivity to the environment. Additionally, the genome- 
wide genetic findings identify associations that also include underlying GxE [87]. In fact, evidence for GxE in $A D$ has recently started to accumulate. For example, an interaction between the APOE4 allele and serum cholesterol levels has been shown to increase the risk of $A D$ [99, 100]. Significant statistical interactions, increasing the risk for developing $A D$, were also found for moderate consumption of alcohol and the APOE4 genotype, as well as for smoking and the APOE4 genotype [101, 102]. Furthermore, an interaction with this risk genotype and social factors, such as cohabiting with a partner has been found; APOE4 carriers who lost their partner before midlife showed an increased risk of developing $A D$, compared to married or cohabiting people [103].

These epidemiological studies indicate that it makes sense to focus future clinical AD studies on measuring both genes and environment and analyzing possible interactions, given that certain environmental factors may only affect a phenotype when the person is genetically endowed. A major drawback of epidemiological clinical studies is that they may indicate merely statistical interactions and thus cannot easily decipher the biological mechanisms that underlie the observed statistical interactions of risk factors. Other major obstacles in clinical studies are the heterogeneity of the study population and co-occurrence of various environmental exposures in the same individuals. Experimental animal research has the advantage of enabling strict control of genetic and environmental variables. Recent advances in transgenesis allow altering specific genes in isolation, and in a time- and regionspecific manner. As such, transgenic mice form a useful tool to study the effects of genetic and environmental variations, and to identify the biological mechanisms that underlie the statistical GxE interactions observed in epidemiological studies (see Figure 1).

\subsection{Transgenic mouse models of $A D$}

Without the intention of giving a full overview of the available AD mouse models, some details on the types of transgenic mice that are discussed in the present review can be found in Table 1. Information on the promoters used for the transgenic construct, and further details on genetic background are not further discussed here as it lies outside the scope of this review.

It is noteworthy that the $A \beta$ sequence of WT rodents has a three amino acid difference compared to humans, making it less likely to aggregate and deposit into amyloid plaques [104]. Therefore, to study $A \beta$ aggregation and plaque formation in rodents it is necessary to manipulate them genetically [104]. Most transgenic mouse models focus on overexpressing human APP, PS1, tau, or APOE variants. 


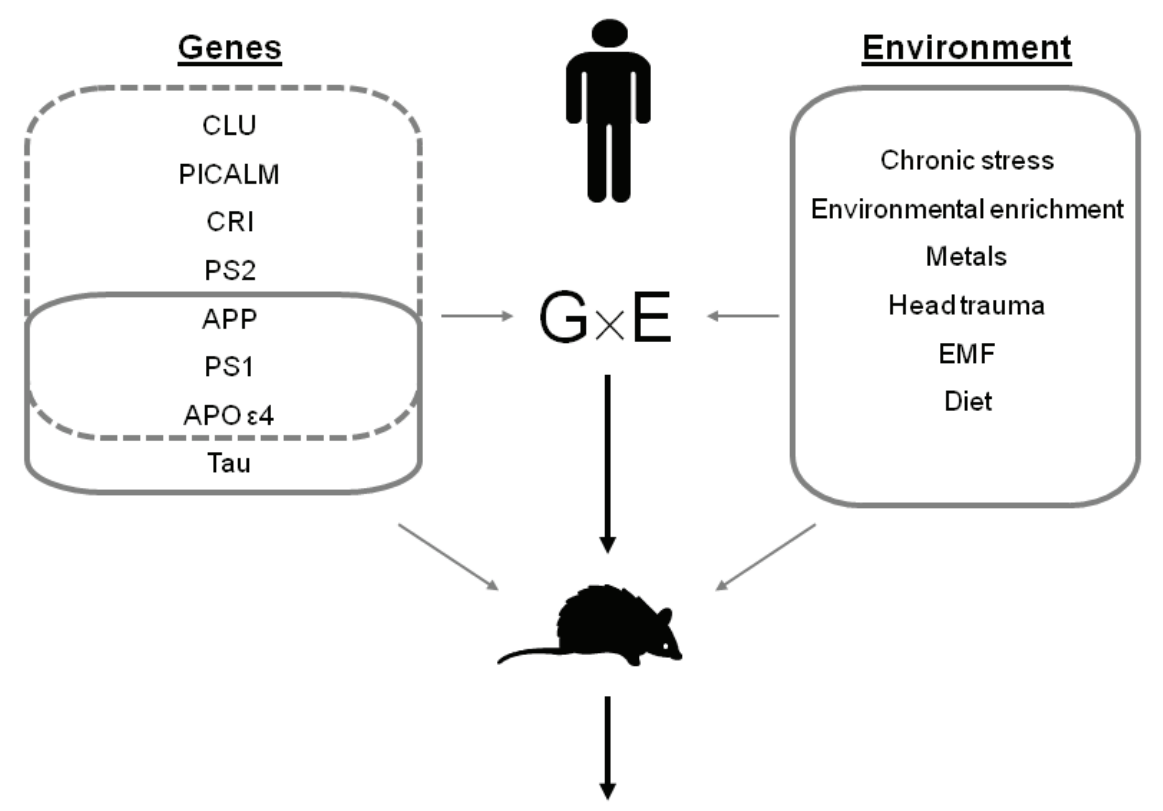

Behavioral endophenotypes and AD-like pathology

Figure 1: Research in Alzheimer's disease (AD) uses both clinical (human) and preclinical (mouse) methods to elucidate the underlying mechanisms of $A D$ etiology. Epidemiological findings, such as genetic and environmental risk factors, can provide tools for investigating their effects on AD etiology separately in mouse models of $A D$. In this review, however, it is postulated that $A D$ research should move towards a gene-environment $(\mathrm{GxE})$ interaction approach, so that the synergistic participation of genes and environment can be scrutinized. Genes in the dashed box represent those genes found to be implicated with Alzheimer's disease etiology in humans, while genes in the solid box resemble the genes that are currently used in mouse models of Alzheimer's disease. APOE4: Apolipoprotein $\varepsilon 4$; APP: amyloid precursor protein; CLU: clusterin; EMF: electromagnetic field; PICALM: phosphatidylinositol binding clathrin assembly protein; PS1: Presenilin 1; PS2: Presenilin 2.

\section{Chronic stress}

\subsection{Human studies of chronic stress}

Chronic stress has been implicated in the etiology of AD. The likelihood of developing $A D$ has been shown to increase by a factor 2.7 with the personality trait distress proneness [72, $73,104]$. Moreover, AD patients show elevated plasma cortisol levels [105, 106] with higher levels of plasma cortisol being associated with a more rapid disease progression and cognitive deterioration [106, 107]. 
Sustained elevated levels of glucocorticoids can cause volumetric and dendritic changes in the hippocampus of rats, mice, and tree shrews [108-111], decrease neurogenesis and impair long-term potentiation [108, 112, 113]. It has therefore been proposed that alterations in HPA-axis functioning might also contribute to the etiology of AD [114-116].

Evidence from studies over the last 20 years indicates that major depression may serve as a risk factor for developing $A D$ [74, 117-123]. A lifetime history of depressive episodes doubles the chance of developing $A D$ [124]. Interestingly, patients with major depression show a cerebrospinal fluid (CSF) profile of $A \beta$-species that resembles the profile seen in $A D$. They display decreased levels of $A \beta 42$ and a decreased $A \beta 40: A \beta 42$ ratio [125], which are considered putative biomarkers for $A D$ [126]. In addition, the severity of depression correlated with binding of 2-(1-\{6-[(2-\{ $\left.{ }^{18} \mathrm{~F}\right\}$ Fluoroethyl)(methyl)amino $]-2-$ naphthyl\}ethylidine)malononitrile, also known as FDDNP, a tracer that binds to plaques and tangles, in the temporal lobe [127]. Moreover, more plaques and tangles in the hippocampus as well as a more rapid cognitive decline have been observed in AD patients with a lifetime history of major depression compared to patients without such history [128]. In contrast, others have suggested that major depression does not function as an independent risk factor for $A D$, but should merely be viewed as an $A D$ prodrome $[117,129,130]$.

\subsection{Animal studies of chronic stress}

Several paradigms have been used to model the effects of chronic stress in mouse models of $A D$. The paradigms that have been applied most frequently are chronic isolation stress and chronic restraint or immobilization stress. Table 2 summarizes the effects of stress exposure in transgenic mouse models of AD.

Chronic isolation stress by subjecting mice to either 3,5 , or 6 months of social isolation from weaning has thus far only been used in the Tg2576 mouse model of $A D$ [78, 131, 132]. This resulted in elevated levels of soluble $A \beta 40$ and $A \beta 42$ up to $59 \%$ and increased plaque deposition in the hippocampus and the neocortex [78, 131]. Moreover, this stress exposure paradigm caused a rise in basal plasma corticosterone levels, paralleled with an increased expression of the glucocorticoid receptor (GR) and corticotropin-releasing factor (CRF) receptor 1 [131]. In addition, impaired contextual memory and decreased cell proliferation in the hippocampal dentate gyrus was observed. Interestingly, the effects of isolation stress on memory deficits and cell proliferation in the dentate gyrus could be prevented by a 14-day treatment of fluoxetine [78].

Another, widely used stress paradigm is restraint stress. Acute short-term restraint stress can already generate elevations in intracerebral interstitial A $\beta$ levels in Tg2576 mice [132], and elevate stress-induced corticosterone release in APPswe mice [133]. Administering CRF or a CRF-antagonist indicated that the interstitial rise in $A \beta$ depended on CRF levels [132]. Acute restraint stress furthermore resulted in a $175 \%$ increase in blood glucose levels in APPswe mice, suggesting a wide impact on metabolism [133]. 
Chronic restraint stress has so far been performed in 3 different mouse models of AD: APPV717I-C100, Tg2576 and PS1-L286V mice. Applying chronic restraint stress to APPV717I-C100 and Tg2576 mice generally resulted in an increased $A \beta$ plaque load, increased $A \beta 40$ and $A \beta 42$ levels, increased tau phosphorylation and increased basal plasma corticosterone levels [134-136]. Chronic restraint stress applied to APPV717I-CT100 mice additionally induced cognitive impairment as measured for example by using cued food, i.e powdered chow mixed with a certain aroma, in the social transfer of food preference task [134]. Chronic restraint stress has also been associated with neuropathological alterations in $A D$ mouse models. PS1-L286V mice exposed to chronic restraint stress displayed elevated numbers of degenerating neurons and a decreased number of proliferating cells in the hippocampus as compared to non-exposed mice [135]. Chronic restraint stress in APPV717I-CT100 mice caused elevated numbers of pyknotic cells in the hippocampus [134] and a reduced dendritic arborization of cortical neurons in Tg2576 mice [136].

Another method to assess the effects of stress is by mimicking the physiological stress response by administering synthetic glucocorticoids, such as dexamethasone, for 7 days. Application of this approach in 3xTg mice resulted in elevated $A \beta$ - and tau-immunoreactivity in the hippocampus, amygdala and neocortex and increased levels of insoluble $A \beta 40$ and $A \beta 42$ and total APP, $\beta$-site of APP cleaving enzyme (BACE1) and APP fragment C99 levels in brain homogenates [137].

\section{Environmental enrichment}

\subsection{Human studies of environmental enrichment}

A reduced risk for $A D$ and a slower rate of cognitive decline have been observed in people having a greater purpose in life and higher levels of physical activity [138, 139].

\subsection{Animal studies of environmental enrichment}

In the field of animal research, the environmental enrichment (EE) paradigm is frequently used to manipulate physical activity and social interactions. By introducing mates (social interaction) and/or toys (physical activity) into the cage of the rodent [140], this paradigm stimulates cognition as well as sensory and motor behavior with concomitant intracerebral cellular and molecular changes [141, 142]. To examine the effect of EE in AD several different paradigms have been imposed on various mouse models of $A D$. Table 3 summarizes the effects of $E E$ in transgenic mouse models of $A D$.

\subsubsection{APP mice}

$\mathrm{EE}$, in terms of housing multiple mice in a larger cage with platforms, running wheels, toys, and other novel habitats, for a period of 6 months improved cognition in a battery of tests 
such as Morris water maze (MWM), circular platform, platform recognition and radial arm water maze, despite signs of stable $A \beta$ deposition in 16-month-old APPswe mice [143]. EE for 4 months in 5-month-old TgCRND8 mice did not significantly alter soluble levels of $A \beta$ in the brain or the blood, but did enhance mRNA expression of angiogenic genes [144]. EE in this mouse model attenuated age-related reductions in cell proliferation, neurogenesis and synaptic plasticity [145], while the same paradigm in another laboratory elevated $A \beta$ plaque load without compromising behavioral phenotypes such as feeding and drinking pattern, grooming, locomotion or cognition [146, 147]. In an attempt to disentangle the exact components of EE that influence phenotypes in APP mutant mice, Wolf et al. [148] exposed APP23 transgenic mice to either an enriched environment or unlimited access to a running wheel and compared both conditions with standard housing. EE had differential effects upon improving performance in the MWM as compared to the increased physical activity and standard housing groups, however, no differential effects on plaque in the neocortex or hippocampus were found [148, 149]. Moreover, mice exposed to EE exhibited signs of increased hippocampal neurogenesis and neurotrophic support [148, 149].

\subsubsection{APP/PS1 mice}

When comparing social interaction and physical activity, differential effects of EE can be observed on learning and memory processes, $A \beta$ plaque load and synaptophysin immunoreactivity of 9-month-old APP/PS1 transgenic mice [150]. EE in APPswe/PS1 1 E9 mice reduced cortical and hippocampal $A \beta$ deposition with mice being more active in the running wheel showing an even more marked decrease in $A \beta$ [151]. Furthermore, $E E$ in PS1/PDAPP mice attenuated cognitive impairments [152].

Possibly in contrast with overt beneficial effects, 2-month-old female APPswe/PS1 $\triangle E 9$ mice exposed to EE for several months displayed increased $A \beta$ levels in the neocortex and hippocampus [153]. After further backcrossing these mice to a C57BI6 background strain in order to attain fewer genetic background differences, the same group demonstrated that EE in 2-month-old transgenic APPswe/PS1 1 E9 female mice, attenuated cognitive deficits [154] but still exhibited a $25 \%$ increase in $A \beta$ deposits in cortical and hippocampal brain regions [154]. One could argue that enhanced secretion and deposition of toxic soluble $A \beta$ species (scavenging the toxic species into packages away from intracellular and synaptic compartments) may be a mechanistic explanation for these findings.

\subsubsection{APOE mice}

EE in mice carrying the APOE3 allele improved learning and memory, as assessed with the T-maze test, while it had no effect in the ones carrying the E4 allele. The improved cognitive performance in APOE3 mice was associated with increased neocortical and hippocampal synaptophysin- and nerve growth factor-immunoreactivity, which was not observed in the APOE4 mice [155]. 
In conclusion, the majority of studies indicate that EE affects AD-related phenotypes in transgenic mouse models of $A D$ pathology, mostly in a beneficial manner, particularly with regards to behavior. However, contradictory results have been reported which can possibly be explained by different experimental paradigms, age, sex, and genetic background of the mice used.

\section{Metal exposure}

\subsection{Lead}

Lead exposure as a risk factor for $A D$ has been proposed by some authors $[156,157]$ while others have argued against it [158]. No studies to date have performed lead exposure experiments in mouse models of $A D$, although other animal work has indicated that lead exposure early in life may contribute to the onset of AD-related pathology later in life [81, 82].

\subsection{Aluminum}

While it has been proposed that occupational aluminum exposure is not a significant risk factor for $A D$ [158], prolonged exposure to aluminum in drinking water is significantly associated with an increased risk of developing $A D$ in a dose-dependent manner with the relative risks varying from 1.00 to 2.14 (for review see [159]). These findings should be regarded with some caution as aluminum concentrations varied highly between the different studies and many variables (such as interaction with other chemical constituents in the drinking water as well as alternative sources of aluminum, for example through antacid use or dietary intake) have often been overlooked in these studies [159].

The only human observational study performed on aluminum intake through diet other than drinking water that altered the risk of $A D$, was performed by Rogers and Simon [160]. Products made of baking-powder often contain high levels of aluminum and AD patients were more likely to have been exposed to ingestion of foods containing baking-powder (retrospectively investigated) than age-matched controls [160]. Other studies suggest that AD patients have significantly enhanced gastrointestinal absorption of aluminum (up to 1.64 times higher) compared to age-matched controls, indicating that differential gastrointestinal function may lead to a systemic rise of aluminum [161, 162].

Pratico and colleagues [163] reported that Tg2576 mice exposed chronically to dietary aluminum displayed increased $A \beta 40$ and $A \beta 42$ levels, plaque deposition and markers of oxidative stress in the hippocampus and neocortex compared to non-exposed Tg2576 mice. Others authors, however, were not able to replicate these data. They reported that chronic aluminum treatment in Tg2576 mice did not affect $A \beta$ load in the cerebral cortex or oxidative stress reactions in the hippocampus, nor impair spatial cognition, as measured by the MWM [163-165]. Aluminum treatment did raise the levels of aluminum and other metals in the 
hippocampus, neocortex and cerebellum, but no major differential effects could be found between Tg2576 and WT mice [164, 166]. The differential effect of aluminum exposure in these mice could possibly be explained by higher concentrations of aluminum in the chow, differential ages at the start of the experiment and a shorter duration of exposure.

\subsection{Iron, zinc, copper}

The endogenous biometals iron, zinc and copper have often been implicated in AD, as they are present in and around amyloid deposits in the AD brain and their presence can promote aggregation of $A \beta$ [167-169].

Although no report has confirmed a direct risk between iron exposure and the risk of developing $A D$, Dwyer and colleagues do propose a ferrocentric model of $A D$ [170]. Higher levels of ferritin iron in the basal ganglia have been considered a risk factor for AD [171, 172]. AD patients show elevated levels of iron in the hippocampus [173] and this metal seems to concentrate in the core and rims of plaques in the amygdala [174].

Rodent research has indicated that gestational or early developmental iron deficiency can alter the expression of the APP and clusterin genes implicated in synaptic plasticity, dendritic outgrowth, and AD pathogenesis [175-177]. Neonatal administration of iron for 3 days to APPswe/PS1 $1 \mathrm{E} 9$ mice was found not to alter $\mathrm{A} \beta$ deposition in the hippocampus and temporal cortex at 6 months of age but did cause changes in lipid composition, decreased steady-state levels of oxidative damage markers and increased astrocyte levels in the temporal cortex [178].

Zinc seems to play a double role in AD etiology. While low levels of zinc have been reported to be protective against $A \beta$ formation [179] and metalloproteases, such as neprilysin and insulin-degrading enzyme (IDE), that degrade $A \beta$ are zinc-dependent [180], it has also been found that high levels of zinc elevate $A \beta$ toxicity $[179,181]$ and promote total $A \beta$ aggregation [169]. AD patients displaying higher levels of zinc in hippocampus and amygdala [173, 174] exhibit normal zinc serum levels, but significantly lower zinc levels in CSF compared to matched controls [182]. This could be explained by the binding of zinc to $A \beta$ in the brain parenchyma [183]. However, to our knowledge no reports have been published on putative associations between zinc exposure and risk of $A D$ in the human population. Nonetheless, several studies have investigated the effects of altered zinc intake in $A D$ mouse models.

Stoltenberg et al. [184] reported that lowering zinc by a 3-month dietary deficiency increased the plaque load in APPswe/PS1 $1 \mathrm{E} 9$ mice by $25 \%$, without changing zinc ion distribution, zinc transporter mRNA expression levels nor inducing oxidative stress [184]. Alternatively, administrating zinc to TgCRND8 and Tg2576 mice through the drinking water for a period of 5 and 9 months, respectively, lowered the amyloid plaque burden in the hilar and molecular region of the dentate gyrus, while impairing spatial memory in MWM [185]. Concurrently, long-term administration of high zinc concentrations in $\mathrm{TgC} 100$ mice did not significantly affect soluble $A \beta$ levels or levels of glial fibrillary acidic protein (GFAP), 
superoxidase dismutase 1 , APP, $\beta$-secretase-cleaved carboxyl-terminal fragment, or neurofilament 200, a marker for neuronal damage [186]. Interestingly, a genetic reduction of zinc in the brain of Tg2576 mice, by crossing these mice with a zinc transporter 3 deficient mouse $\left(\mathrm{ZnT}^{-/-}\right)$, significantly reduced the plaque load in the hippocampus and neocortex while increasing the ratio between soluble versus insoluble $A \beta$ [187].

$A D$ patients have been shown to display reduced copper levels in the amygdala and hippocampus, while copper levels are specifically elevated in amyloid plaques [173, 174]. Copper intake in AD patients decreases the reduction of AB42 in CSF most typically seen as the disease progresses, but does not ameliorate cognitive performance [188]. Adding copper to drinking water of cholesterol-fed rabbits causes accumulation of $A \beta$ and the formation of plaque-like structures [189].

Exposing $A D$ mouse models to chronic copper administration has yielded conflicting results. Chronic copper administration to APP715SL mice did not alter copper, zinc, iron, A $\beta$ nor APP levels in the brain [190]. Long-term administration of high levels of copper resulted in a $18 \%$ decrease in soluble $A \beta 40$ and increased zinc levels in the brain without changing GFAP, SOD1, APP, C100 or NF200 levels to TgC100 mice. Yet, copper exposure in 3xTg mice led to elevated steady-state levels of APP and C99 as well as to increased A $\beta$ production and tau phosphorylation in the brain [191]. Interestingly, copper, APP and A $\beta$ seem to be closely connected; Tg2576 mice displayed an overall reduction of copper in the brain, whereas the ablation of APP and amyloid precursor-like protein 2 increased overall central copper levels [192, 193].

In summary, both human and rodent research on the exact contributing roles of environmental metal exposures in interaction with AD risk genes APP and PS1 remain largely inconclusive. For an overview of metal exposure in the transgenic animal models of AD listed above, see Table 4.

\section{Traumatic brain injury}

\subsection{Human studies of traumatic brain injury}

Traumatic brain injury ( $\mathrm{TBI}$ ) has repeatedly been identified as a risk factor for $A D$. It has been suggested that $T B I$ accelerates the onset of $A D$ and that the severity of the injury increases the risk of $A D$ [194]. AD-like pathology has been observed after acute brain trauma, even in brains of young individuals. A polymorphism in the promoter of the gene that encodes neprilysin, causing a greater length in GT repeats, has been associated with the acute development of plaques following TBI [195]. In addition, carriers of the APOE4 genotype have been associated with poorer outcome after TBI [194]. 


\subsection{Animal studies of traumatic brain injury}

For an overview of TBI in mouse models of $A D$, see Table 5. After corticol contusion, 10- to 16-month-old PDAPP mice did not show significant differences in behavior or $A \beta$ neuropathology following TBI, as compared to WT controls that underwent the same procedure of experimental brain injury [196]. Inducing TBI in the PDAPP mouse model at 4 months of age, accelerated memory loss as assessed with the MWM test. TBI also resulted in hippocampal neuronal loss one week after injury, which was associated with an increase in hippocampal $A \beta 40$ and $A \beta 42$ [197]. Furthermore, TBI resulted in long-term effects at 2, 5, and 8 months after TBI: a significant reduction in $A \beta$ plaque load was found which was accompanied with more pronounced hippocampal atrophy. TBI induction in APP ${ }^{\text {NLh/NLh }}$ mice caused a twofold increase in soluble hippocampal $A \beta$ levels at 3 and 7 days after TBI. Additionally, post-TBI administration of caspase- 3 inhibitors and the hypolipemic simvastatin were able to attenuate impaired hippocampal synaptic function, microglial activation and MWM performance after TBI induction in APP ${ }^{\text {NLh/NLh }}$ mice $[198,199]$. TBI induced by cortical impact provoked gene expression changes in 22-month-old APPswe mice compared to WT mice. Expression changes were detected in genes involved in various biological pathways such as immune response, cell cycle and cell death, cellular development, tissue development and connective tissue function and development, cellular movement, and hematological systems [200].

Single and repetitive mild TBI, using a cortical impact device, in 9-month-old Tg2576 mice as compared to sham treated transgenic and WT, resulted in significant cognitive dysfunction (measured with MWM) without affecting motor performance 16 weeks after TBI. However, only repetitive TBI caused increased $A \beta$ burden in the hippocampus and neocortex with a parallel increase in isoprostane, an indicator for increased oxidative stress [201]. TBI in 10-month-old transgenic mice over-expressing either human APOE3 or 4, was associated with differential gene expression, particularly in genes related to oxidative stress, with an increased expression of anti-oxidant genes in the APOE3 mice as compared to the APOE4 [202].

Thus, it is evident that $T B I$ is detrimental for $A D$ pathology and related cognitive performance and more research is needed to elucidate the interaction of the APOE gene and brain trauma.

\section{Electromagnetic field exposure}

Occupational exposure to electromagnetic field (EMF) has been proposed as a risk factor of $A D$. In particular, extremely low frequency exposure has been implicated to increase the odds to develop AD up to 2.03 (as reviewed in [202]). Strikingly, Arendash et al. [203] demonstrated that long-term high frequency exposure to EMF (i.e., similar to that generated by cell-phone use) was beneficial to AßPPswe mice (see Table 4). EMF exposure in young adult $A \beta P P s w$ mice prevented the age-related genotype-specific cognitive impairment, while 
EMF in aged ABPPsw mice was also able to reverse cognitive impairment in these animals. Chronic EMF exposure furthermore influenced $A \beta$ aggregation in the brain, with higher levels of soluble $A \beta$ and less $A \beta$ plaques in the hippocampus and entorhinal cortex of A $\beta P P s w$ mice. EMF exposure has been proposed to contribute to a decrease in $A \beta$ aggregation, via altering levels of transthyretin[204]. Transthyretin is known to sequester $A \beta$ in CSF, thereby hindering its aggregation into amyloid plaques [204]. Interestingly, AD patients show a significant decrease in CSF transthyretin levels [205] while increased transthyretin levels have also been found in blood serum of long-term wireless phone users [204]. Thus, effects of EMF are quite puzzling while the association with AD remains to be firmly established.

\section{Effects of diet and nutritional factors}

\subsection{Mediterranean diet}

Various dietary and nutritional factors seem to be protective or detrimental in the development and course of AD. One of the most prominent is the Mediterranean type of diet which has been linked to reduced risk of developing $A D$ and showing a dose-response effect (high adherence to Mediterranean diet, OR: 0.76; moderate adherence, OR: 0.47) [206-208].

A typical Mediterranean diet is characterized by higher consumption of vegetables, fruits, cereals, fish, olive oil, and associated with a general higher consumption of unsaturated fatty acids and lower consumption of saturated fatty acids, usually accompanied by mild or moderate alcohol intake (preferably red wine taken with meals) [206]. The exact factors and mechanisms by which the Mediterranean diet is protective remains to be elucidated, although it has been speculated that this diet can attenuate the detrimental effects of oxidative stress and inflammation [207].

\subsection{Western diet and Obesity}

Obesity during mid-life is associated with an increased risk for $A D$, with an $O R$ of 2.4 , additively increasing up to 6.2 when combined with high total cholesterol levels and high systolic blood pressure [209]. Higher intake of calories and fat have been associated with increased risk for developing AD, particularly in APOE4 carriers, with a hazard ratio of 2.3 [210]. Western, high-fat and low carbohydrate diet for 4 months in 1- month-old Tg2576 mice, increased levels of soluble $A \beta$ in brain homogenates, while the treatment did not have any effect on plaque load [211]. Further, insulin resistance induced by 5 months of high-fat diet, in 9-month-old Tg2576 mice, led to a twofold increase of $A \beta 40$ and $A \beta 42$ peptide content in the hippocampus and a twofold increase in plaque burden in the neocortex, with a concomitant acceleration of cognitive decline as measured by the MWM. In addition, $\mathrm{Y}^{-}$ secretase activity was increased, while the expression of IDE was decreased by this diet [212]. APP/PS1 KI mice exposed to Western, high-fat diet showed increased oxidative 
stress markers as measured in brain homogenates of 2-month-old mice when compared to non-transgenic controls, but $A \beta$ levels were not altered [213]. In another study, Western diet increased $A \beta$ deposition in the hippocampus of the APPswe/PS1 $\triangle E 9$ transgenic mice at 18 months of age, after a period of 12 months on a high fat diet [214]. In the 3xTg mouse model of $A D$, a high fat diet starting at the age of 4 months for a total period of 13 months, induced similar effects in the frontal cortex [215].

\subsection{Cholesterol}

As the generation, deposition and clearance of $A \beta$ is regulated by cholesterol, many studies have specifically focused on the implication of lipids, cholesterol metabolism, related vascular disease, APOE genotype and their interrelationships on the development of $A D$ [216-218]. The precise mechanisms underlying cholesterol and APOE4 need further investigation, as it is not clear whether cholesterol and the APOE4 genotype act as independent factors or interact with one another or whether the effect of APOE4 is partially mediated by high cholesterol levels [217-220]. Also, hypercholesterolemia in 3-month-old $\mathrm{APP}_{\text {swe }} / \mathrm{PS} 1_{\mathrm{M} 146 \mathrm{~L}}$ mice has been shown to accelerate $A \beta$ accumulation while drug- induced hypocholesterolemia reduced the amyloid pathology [221, 222].

\subsection{Docosahexaenoic (DHA)}

Studies in mouse models of AD amyloidosis, such as Tg2576, APPswe/PS1 $\triangle E 9$, and 3xTg, have shown that a diet rich in the omega- 3 fatty acid DHA reduces $A \beta$ accumulation and somatodendritic tau accumulation, improves cognition, and induces cerebral hemodynamic changes [214, 223-226]. Such findings are in line with evidence from epidemiological studies showing a protective effect of diets rich in omega-3 fatty acids [227-229]. More specifically, DHA-enriched diet was shown to increase relative cerebral blood volume with a concomitant improvement in spatial memory and reduction of $A \beta$ load in $A P P_{\text {swe }} / P S 1_{M 146 L}$ mice [230]. Exposing APPswe/PS1 $\triangle E 9$ mice to a diet high in omega-3 fatty acids, however neither improved cognition in APPswe/PS1 $\triangle \mathrm{E} 9$ mice nor reduced hippocampal $A \beta$, but increased omega-3 fatty acid levels in their brain [231]. Interestingly, high levels of omega- 6 were linked to cognitive impairment [231].

\subsection{Vitamins}

Dietary deficiency of B6, B12, and folate for 7 months, increased $A \beta$ levels in the brains of 15-month-old Tg2576 mice, without altering APP, BACE-1, A disintegrin and metallopeptidase 10 (ADAM-10), nicastrin, IDE, APOE or neprilysin [232]. Additionally, the same pattern of dietary vitamin B deficiency, led to increased expression of PS1 via DNA demethylation of the promoter region of the encoding gene in brain homogenates of TgCRND8 mice [233]. In the brains of mice of the same animal model, vitamin B deficiency increased the levels of glycogen synthase kinase $3 \beta$ (GSK3 $\beta$ ) and reduced the activity of protein phosphatase $2 \mathrm{~A}$, which are both involved in the hyperphosphorylation of tau [234]. 
Moreover, folic acid deficiency for a 3-months in APPswe mice, did not affect the A $\beta$ plaque load, but induced neuron loss in the CA3 region of the hippocampus and enhanced hippocampal DNA damage, as compared to controls [235]. Besides B6 and B12, deficiency of $B 1$, also called thiamine, exacerbated $A \beta$ pathology via an upregulation of BACE1 in brains of Tg19959 mice [236].

Furthermore, dietary supplementation with the coenzyme Q10 for 2 months, delayed hippocampal atrophy in 22-month-old $\mathrm{APP}_{\text {swe }} / \mathrm{PS}$ Leu235Pro $_{\text {mice }}$ as compared to vehicle treated controls [237, 238], with concurrent reduction in plaque load [237, 238]. Deficiency of vitamin $A$ has been implicated in $A \beta$ accumulation, loss of long-term potentiation and memory impairment, while administration of its active metabolite retinoic acid for a duration of 2 months, was able to rescue these deficits in the frontal cortex and hippocampus of 7 month-old APPswe/PS1 $1 \Delta 9$ transgenic mice [239].

\subsection{Caffeine, green tea}

Besides the various nutritional factors, other lifestyle habits have also been associated with $A D$. Longitudinal studies have shown that coffee and tea drinking are associated with decreased risk for cognitive decline, dementia and $A D$ in various population samples [240, 241]. Another study showed a protective effect of caffeine only in women, with a relative risk of 0.49 , [242] but a meta-analysis estimated an overall protective effect against dementia, with a relative risk of 0.84 , though pointing out the large heterogeneity in the methods of the various epidemiological studies [243].

Acute and long-term caffeine consumption was recently shown to delay cognitive decline and lower $A \beta$ pathology in the hippocampus of 15-month-old APPswe and $\mathrm{APP}_{\text {swe }} / \mathrm{PS} 1_{\mathrm{M} 146 \mathrm{~L}}$ mice, by suppressing $\beta$ - and $\gamma$-secretase levels $[244,245]$ Furthermore, oral or intraperitoneal administration of epigallocatechin-3 gallate, which is derived from green tea, for 2 or 6 months, exerted beneficial effects in APPswe transgenic mice, at the age of 14 months. The beneficial effects consisted of a reduction in $A \beta$ pathology in the neocortex and hippocampus, with a parallel improvement of working memory [246-248]. Furthermore, administration of the citrus-derived flavonoid luteolin and its analogue diosmin for a total of 30 days, significantly reduced $A \beta$ pathology in the hippocampus and neocortex of 9-month-old Tg2576 mice. This effect was mediated via an inhibition of GSK3 $\beta$, which increased PS1 phosphorylation [249].

\subsection{Wine}

Moderate red wine consumption has been shown to be beneficial. Cabernet sauvignon administration for 7 months in 4-month-old Tg2576 mice attenuated the cognitive impairment that is observed in these mice, in terms of spatial memory, when compared to ethanolconsuming and tap water Tg2576 controls. Cabernet sauvignon consumption decreased cortical and hippocampal $A \beta$ plaque load in these mice, by promoting non-amyloidogenic processing in the direction of $\alpha$-secretase cleavage [250]. Further, in vitro studies in 
hippocampal neuron cultures derived from Tg2576 mice, showed that the polyphenol extracts from Cabernet sauvignon grapes increased the levels of $\alpha$-secretase, which promotes the non-amyloidogenic cleavage of APP that reduced the levels of A $\beta$ peptides [250]. Furthermore, consumption of the muscadine wine was proven to attenuate $A \beta$ pathology in brains of 14-month-old Tg2576 mice, following a 10-month wine treatment, with a different mechanism of action. In this case muscadine consumption reduced the aggregation of $A \beta$, with a parallel improvement in spatial memory [251]. The differential effect of the two types of wine could be attributed to their distinct composition in polyphenolic compounds, which have a differential effect on APP processing [251].

\subsection{Nicotine.}

Smoking in humans has been linked with increased risk for AD [252-254], while nicotine administration for 6 months to transgenic mice carrying the APPswe mutation reduced the levels of insoluble $A \beta$ in various brain regions of 15 -month-old mice $[255,256]$. Nevertheless, nicotine administration for 5 months exacerbated hippocampal tau pathology in 6-month-old 3xTg mice, increasing tau hyperphosphorylation and aggregation, in combination with an earlier onset of these tau-related changes compared to controls [257].

\subsection{Caloric restriction}

Caloric restriction in animals has been found to prolong mean and maximum life span, reduce body fat, attenuate age-related molecular changes and slow the decline associated with aging in various species [258]. In non-human primates, for example, caloric restriction prolongs lifespan and delays the onset of diseases, such as cardiovascular diseases, diabetes, and brain atrophy [259]. Restricting caloric intake by $40 \%$ for 6 weeks in APPswe and 14 weeks in $\mathrm{APP}_{\mathrm{swe}} / \mathrm{PS} 1_{\mathrm{M} 146 \mathrm{~L}}$ mice has been shown to reduce the number and size of amyloid plaques by $40 \%$ and $55 \%$ respectively, while also reducing the plaque-related astrocyte activation. These effects were observed in the neocortex and hippocampus [258]. Similar effects have been observed in the hippocampus of the $3 x \operatorname{Tg}$ model after 14 months of caloric restriction [260].

\subsection{Others}

In line with studies showing a beneficial effect of nonsteroidal anti-inflammatory agents on $A D$ pathology in transgenic models of $A D$ [261], the phytogenic curcumin (a major dietary component in India), administered for 6 months reduced levels of soluble and insoluble $A \beta$, plaque load, oxidative stress and inflammatory response in various brain regions of 16month-old transgenic mice carrying the APPswe mutation [262]. Additionally, blueberry supplementation and Gingko biloba extract treatment have been found to improve memory deficits in APP/PS1 and Tg2576 mice without affecting amyloid plaque load [263, 264]. An overview of all nutritional and dietary factors influencing animal models of $A D$ is given in Table 6. 


\section{Strengths, limitations and future challenges of GxE research in $A D$}

This review provides an overview of experimental mouse data on environmental exposures known to be associated with AD. In general, it may be concluded that many studies have shown effects of environmental manipulations on a wide variety of phenotypes in transgenic mouse models of $A D$.

It is challenging to evaluate the exact role of GxE in the field of preclinical AD research, due to several limitations. Numerous mouse models have been used on different genetic backgrounds and at multiple ages, applying various protocols of experimental exposures. This high level of variability makes it difficult to draw firm and general conclusions on any of the discussed exposures. In addition, the read-out parameters differed per group, with some focusing on $A \beta$ pathology, synaptic integrity or oxidative stress and others emphasizing behavioral effects. Another limitation is that most animal models used, have focused on $f A D$ mutations, while a GxE interaction may be more applicable in sporadic forms of AD.

The experimental design of most of the available studies often consisted of testing the effects of environmental exposures only in transgenic mice, without full comparison of the effects of those environmental exposures in wild type animals. In such an experimental setup, one can merely study disease acceleration or progression as a result of environmental exposure. Additionally, the effects of some environmental factors may have a differential effect in the initiation of the disease from their effect on disease progression. Evidently, this type of research can still provide us with insights on underlying biological mechanisms, but it cannot disentangle the synergistic participation of genes and environment on the induction of an $A D$ phenotype.

In a clinical setting, however, GxE interaction in AD etiology is particularly complex to decipher with standard epidemiological designs, particularly because the time-window between environmental exposures during life and the clinical phenotype of $A D$ is very long. The high variability in environmental exposure across the life span also makes it challenging to capture this interaction.

Most $A D$ research over the past decades has been $A \beta$-centered, yet recent clinical trials based on the amyloid cascade hypothesis have yielded controversial and sometimes disappointing results [265]. Similarly, the majority of animal models of AD have also grossly been focused on $A \beta$-enhancing mutations. Furthermore, outcome measures on the effects on environmental factors are often expressed in terms of soluble and insoluble $A \beta$ and plaque load alterations. Because the extent of amyloid burden does not correlate well with AD symptomatology, one could argue that it would be advantageous to design future studies in a multidisciplinary manner encompassing a wide range of outcome measures such as behavioural phenotypes, biochemical, molecular as well as neuropathological alterations and use similar outcome measures in the various studies conducted by different research groups so that results are more comparable. 
Possible biological mechanisms that mediate the effects of environmental exposures and that could be the focus of further translational $A D$ research are, among others, inflammation, oxidative stress, protein misfolding, glucose metabolism, and epigenetics [98, 266-269]. The AD brain shows ample signs of ongoing inflammatory processes, such as the presence of pro-inflammatory cytokines and activated microglia surrounding amyloid plaques.[270]. A large body of evidence has pointed towards a role of oxidative stress and oxidative damage in brain regions that are affected by AD [266]. In addition, changes in the epigenome have been implicated in the pathophysiology of $A D$ that can be triggered by various environmental factors [98], while the exact role of misframed proteins, such as ubiquitin +1 that have been found to accumulate in $A D$, remains also to be fully elucidated $[269,271]$.

\section{Concluding Remarks}

It appears likely that a large part of, at least, sporadic cases can be connected with GxE interactions, with the field being challenged to identify the most relevant GxE interactions in prospective clinical studies of subjects that will develop AD. Exposure to environmental risk factors during early life has been linked to other complex psychiatric phenotypes and disorders. For example, prenatal maternal and early life stress can be viewed as a risk factor for developing major depression and schizophrenia [272-274]. Likewise, environmental exposures during early life may well impact on the risk of developing AD. For example, neonatal exposure to metal lead has been proposed as an early environmental trigger for AD-related pathology in rodents and macaque monkeys [82]. Findings like these have spurred researchers to formulate the 'Latent Early Associated Regulation' (LEARn) theory of $A D$ pathogenesis proposing that indeed early environmental exposures can change gene expression for long time periods and can induce pathology that only becomes apparent later in life, after subsequent trigger(s) [80, 82].

While numerous challenges lie ahead, it can be argued that it is timely to move attention of epidemiological as well as experimental animal research in the field of AD towards the synergistic approach of GxE research. In a preclinical setting, one could envision focusing more on the use of recently identified genetic variants of the newly found GWAS genes. Second, current studies can benefit from the further technological advances in transgenesis that enable time- and region-specific expression of transgenes, thereby allowing for the investigation of GxE during specific time windows in development and aging, as well as in specific brain regions. Third, It may be valuable to scrutinize the roles of genetic and environmental risk factors (and their interactions) of diseases that have been associated with the onset of $A D$, such as cardiovascular disease and diabetes mellitus type 2. The underlying mechanisms of these disorders, which likely involve GxE, could shed a new light on the etiology of $A D$. 
To conclude, moving towards a GxE approach in both clinical and experimental animal studies seems promising in further elucidating the multifactorial etiology of $A D$, and in identifying modifiable factors that are of particular relevance for subgroups of $A D$ patients. The further use and development of animal models combining genetic and environmental manipulations will be a driving force in elucidating the exact biological underpinnings of this detrimental disorder.

\section{Acknowledgements}

Funds were provided by the International Stichting Alzheimer Onderzoek (ISAO), grant number 09552 to Bart P.F. Rutten and by the Netherlands Organisation for Scientific Research (NWO, Veni Award 916.11.086), by the ISAO, grant number 07551, to Daniel L.A. van den Hove; by a Marie Curie Host Fellowship Grant MC-EST 020589 EURON to Leonidas Chouliaras; and by the National Institutes of Health (NIH) grant number AG05138 to Patrick R. Hof.

\section{Tables}




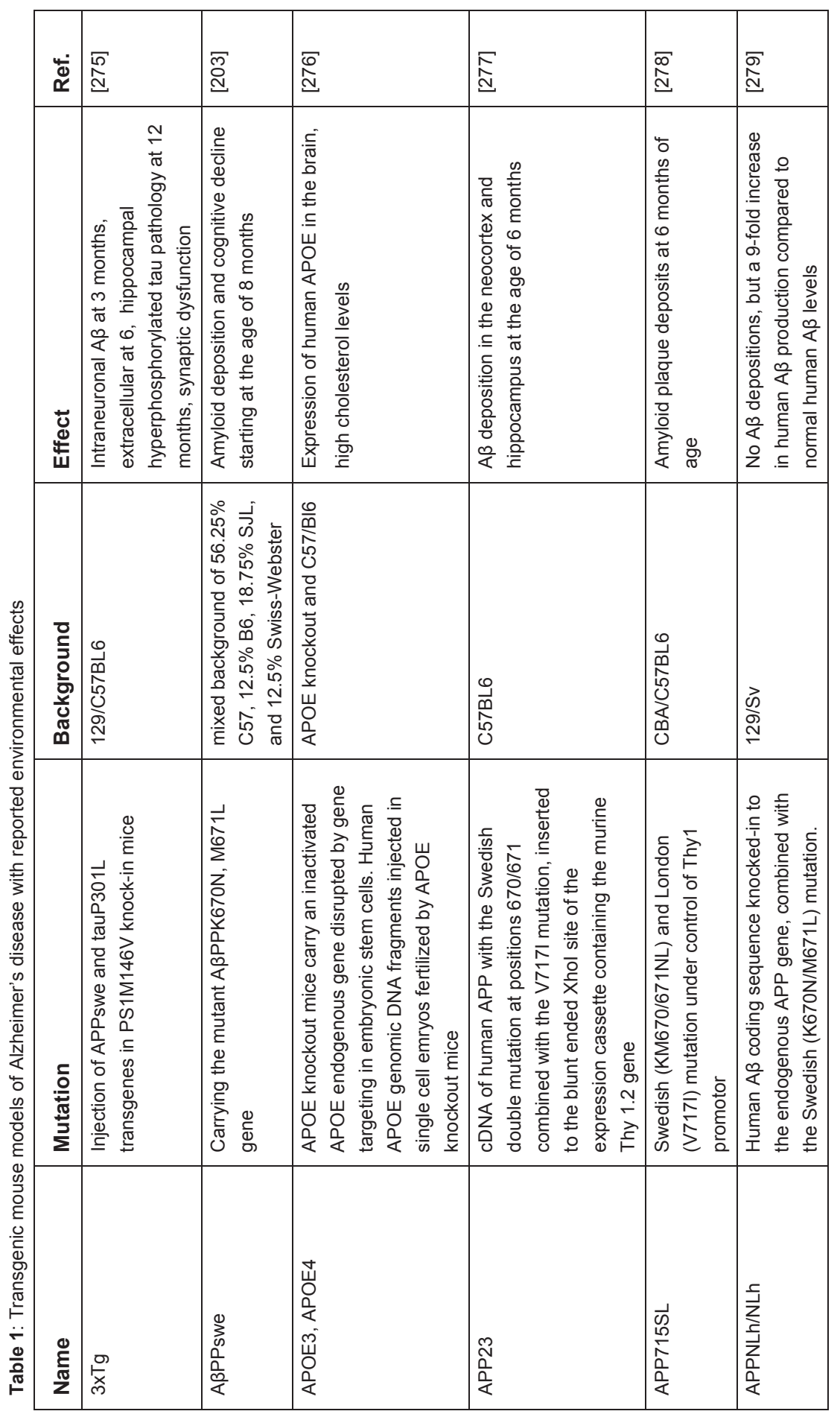




\begin{tabular}{|c|c|c|c|c|c|c|c|}
\hline 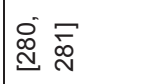 & 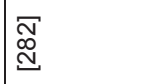 & 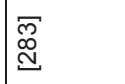 & 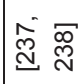 & 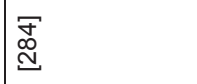 & $\begin{array}{l}\mathscr{D} \\
\stackrel{\infty}{N}\end{array}$ & $\begin{array}{l}\bar{Q} \\
\stackrel{D}{N} \\
\end{array}$ & 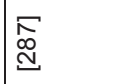 \\
\hline 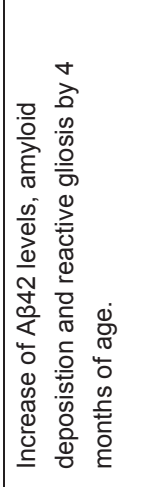 & 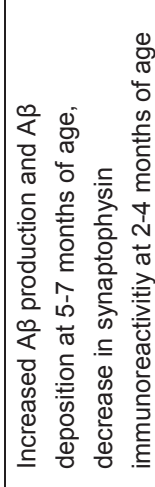 & 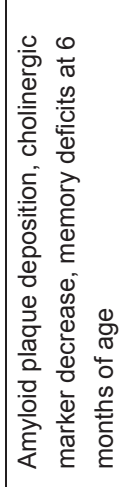 & ' & 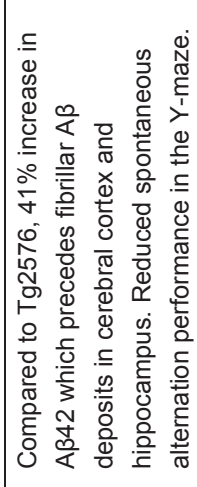 & 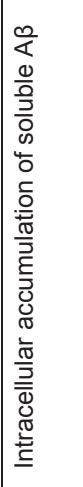 & 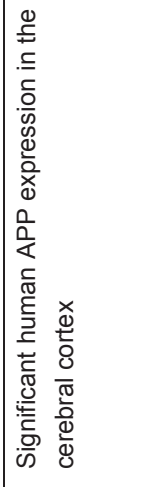 & 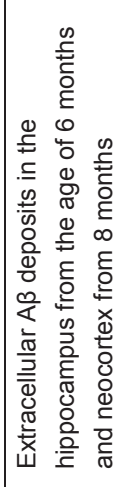 \\
\hline$\frac{\stackrel{D}{V}}{\stackrel{i}{i}}$ & 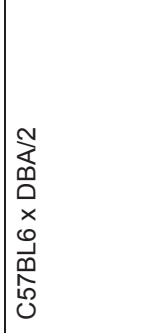 & 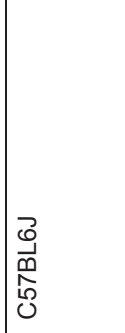 & 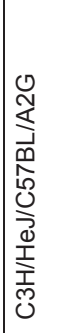 & 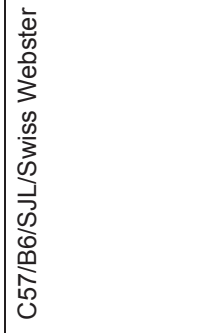 & $\begin{array}{l}0 \\
0 \\
\underline{w} \\
\hat{\omega} \\
0\end{array}$ & $\begin{array}{l}0 \\
\underline{0} \\
\underline{\omega} \\
0 \\
0\end{array}$ & \\
\hline 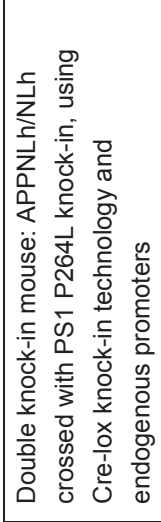 & 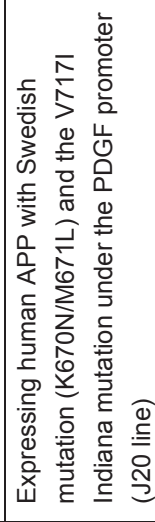 & 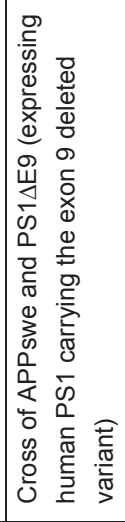 & 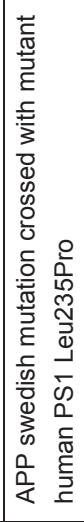 & 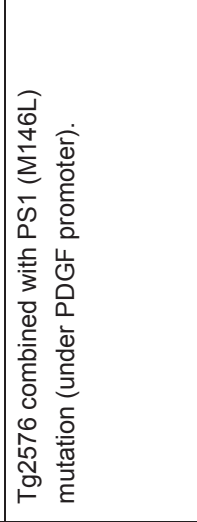 & 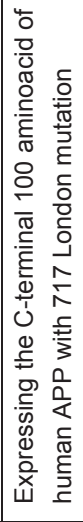 & 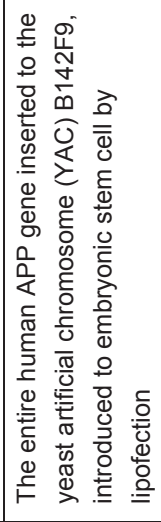 & 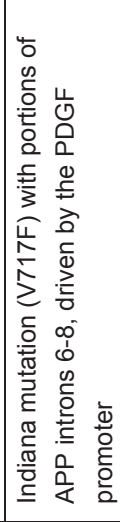 \\
\hline $\begin{array}{l}\bar{x} \\
\bar{n} \\
\frac{\alpha}{\alpha} \\
\frac{\alpha}{\alpha}\end{array}$ & 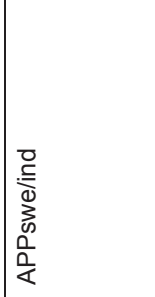 & 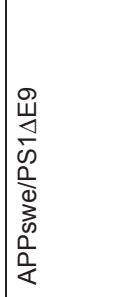 & 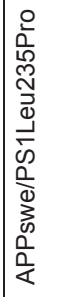 & 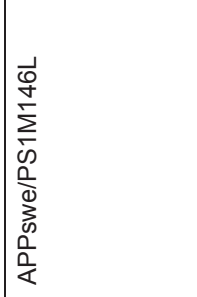 & 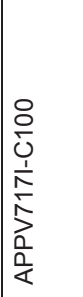 & $\begin{array}{l}0 \\
0 \\
\frac{1}{1} \\
0 \\
\frac{1}{\alpha} \\
\frac{1}{\alpha}\end{array}$ & 高 \\
\hline
\end{tabular}




\begin{tabular}{|c|c|c|c|c|c|c|}
\hline 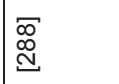 & 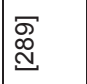 & হ্ষ্ত & 灾 & 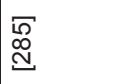 & 胥 & 忍 \\
\hline 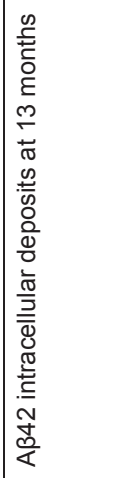 & 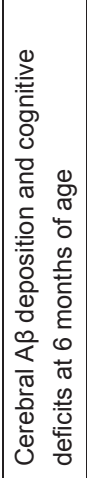 & 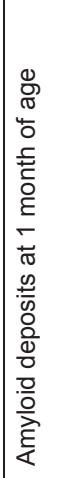 & 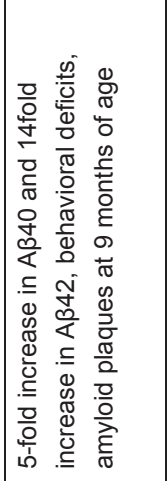 & 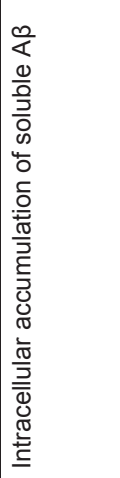 & 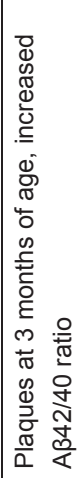 & 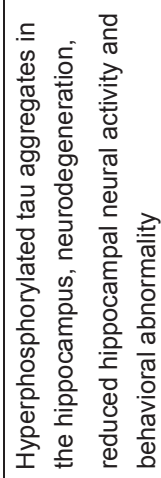 \\
\hline$\sum_{\text {M }}^{Z}$ & $\begin{array}{l}\text { I } \\
\tilde{0} \\
0 \\
\underline{\omega} \\
\hat{b} \\
0\end{array}$ & 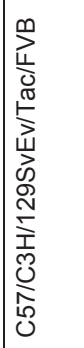 & $\begin{array}{l}0 \\
\underline{p} \\
\underline{w} \\
\hat{b} \\
0\end{array}$ & $\begin{array}{l}0 \\
\stackrel{0}{\alpha} \\
\hat{\omega} \\
\mathcal{U}\end{array}$ & 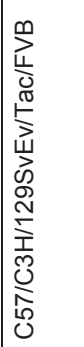 & $\begin{array}{l}\vec{J} \\
\text { D్ } \\
\varnothing\end{array}$ \\
\hline 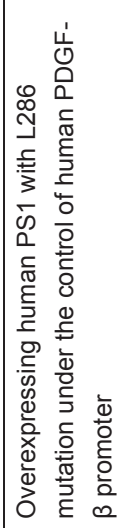 & 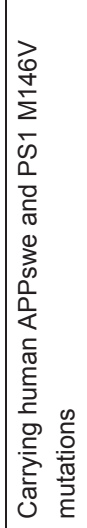 & 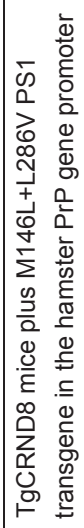 & 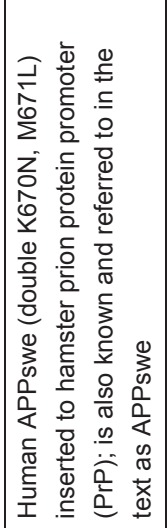 & 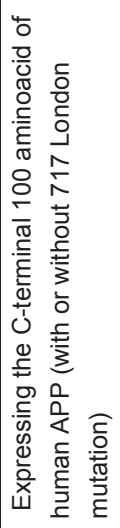 & 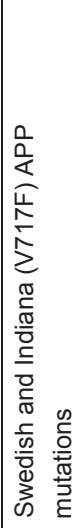 & 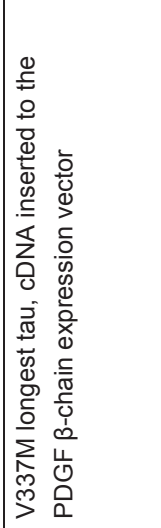 \\
\hline 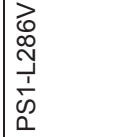 & 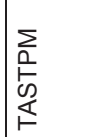 & 吕 & 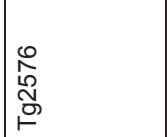 & $\begin{array}{l}8 \\
\frac{0}{0} \\
0 \\
\end{array}$ & 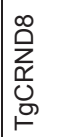 & 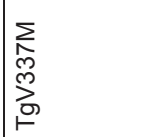 \\
\hline
\end{tabular}




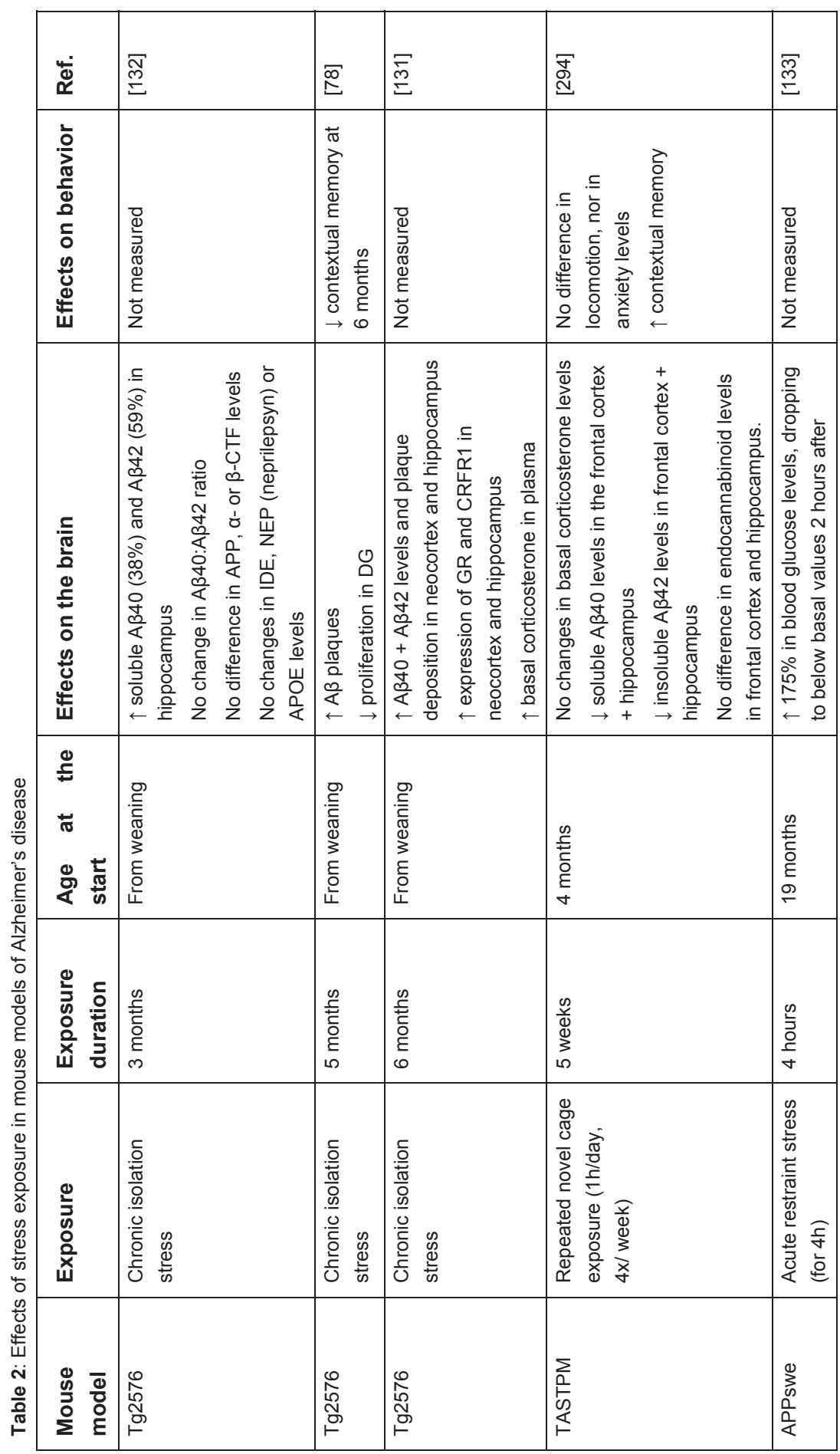




\begin{tabular}{|c|c|c|c|c|}
\hline & 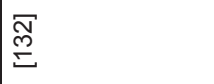 & $\stackrel{F}{\stackrel{F}{巳}}$ & $\stackrel{\text { F }}{\stackrel{\text { I }}{巳}}$ & $\begin{array}{l}\stackrel{\Xi}{\infty} \\
\stackrel{\mathscr{m}}{巳}\end{array}$ \\
\hline & 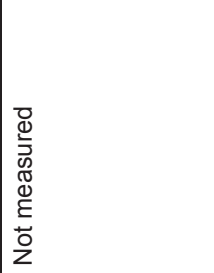 & 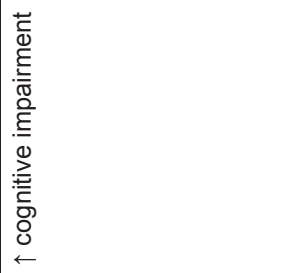 & 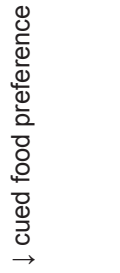 & 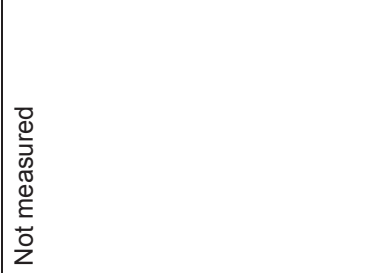 \\
\hline 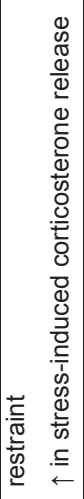 & 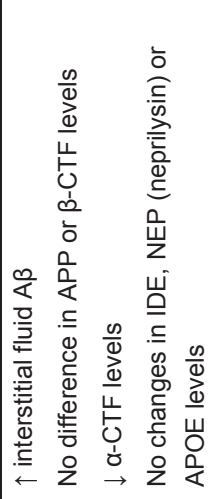 & 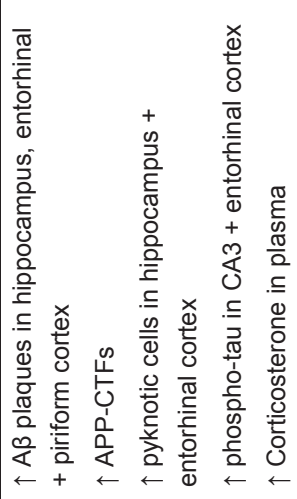 & 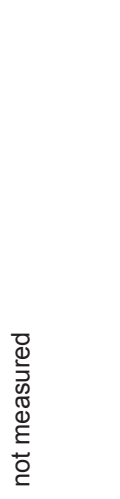 & 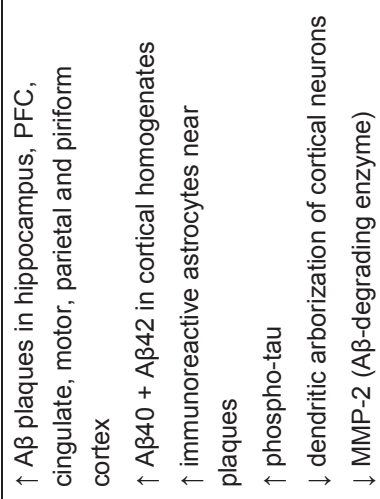 \\
\hline & 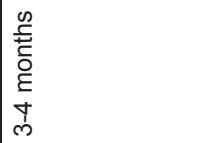 & 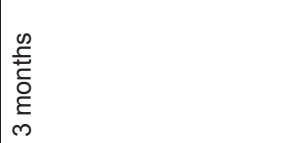 & 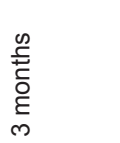 & 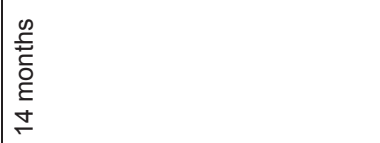 \\
\hline & \begin{tabular}{|l}
$\frac{\infty}{2}$ \\
$\overline{0}$ \\
$\frac{0}{m}$ \\
\end{tabular} & 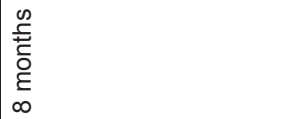 & 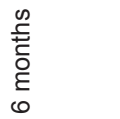 & $\begin{array}{l}0 \\
\frac{0}{0} \\
0 \\
0 \\
0\end{array}$ \\
\hline & 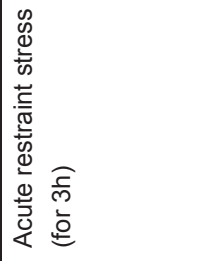 & 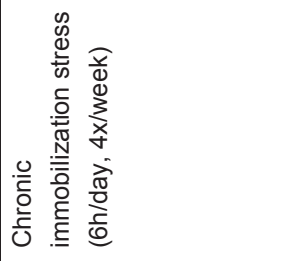 & 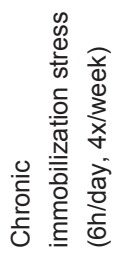 & 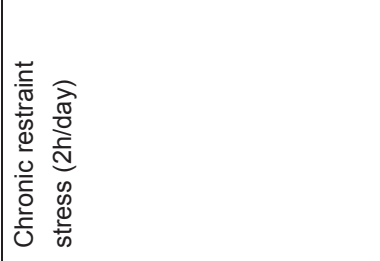 \\
\hline & 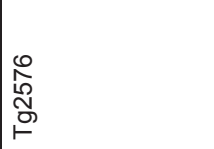 & $\begin{array}{ll}\frac{\pi}{5} \\
\frac{1}{3} & 8 \\
\frac{1}{0} & \frac{0}{5} \\
\frac{n}{4} & \frac{1}{6}\end{array}$ & $\begin{array}{l}\text { N } \\
\text { W } \\
\text { D }\end{array}$ & 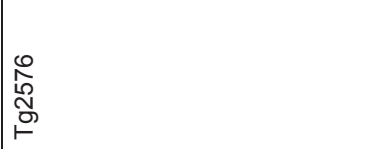 \\
\hline
\end{tabular}




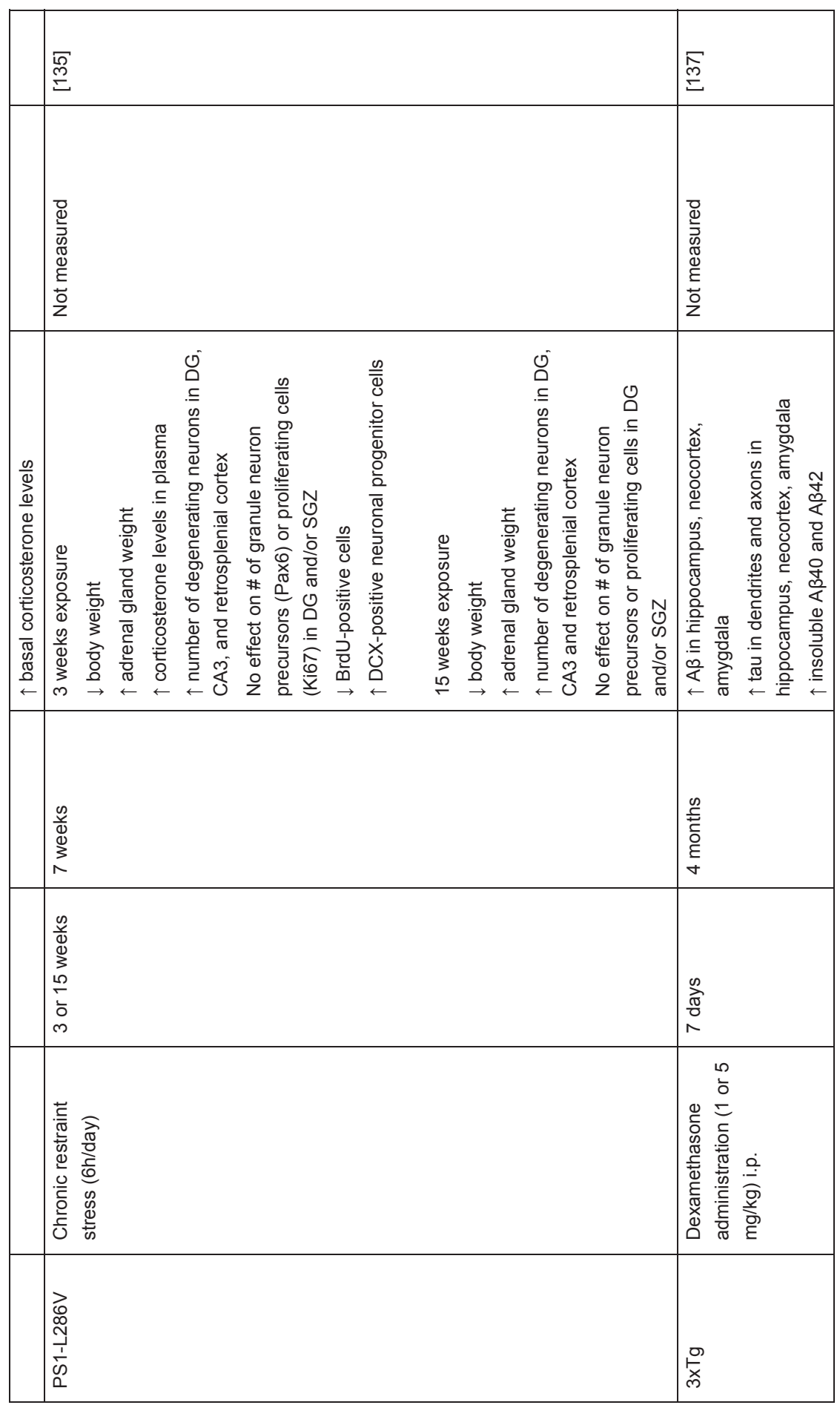




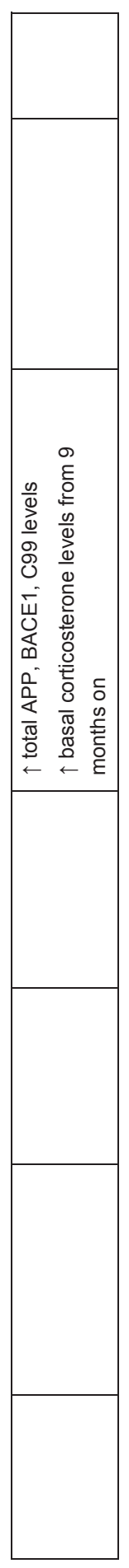




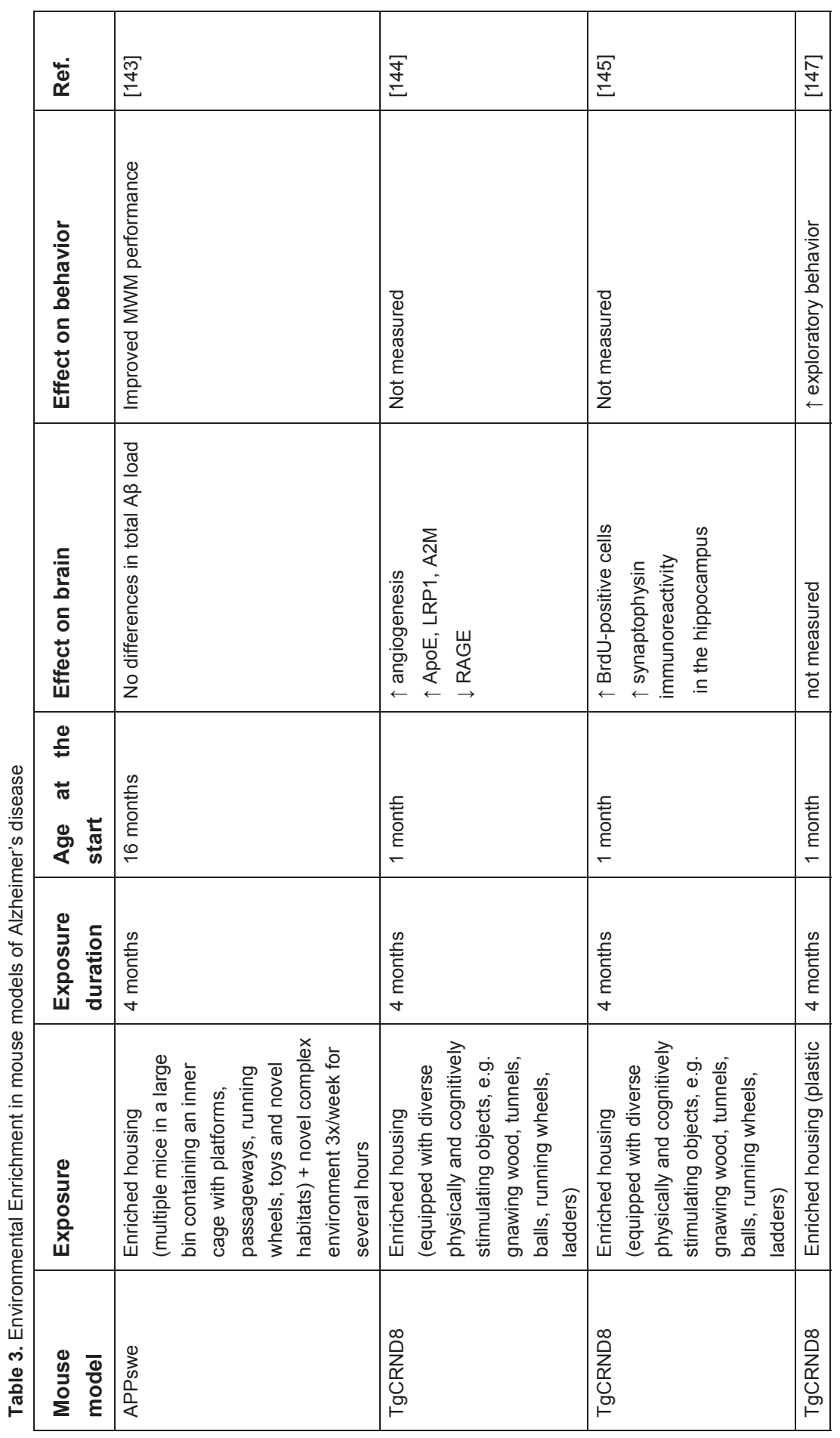




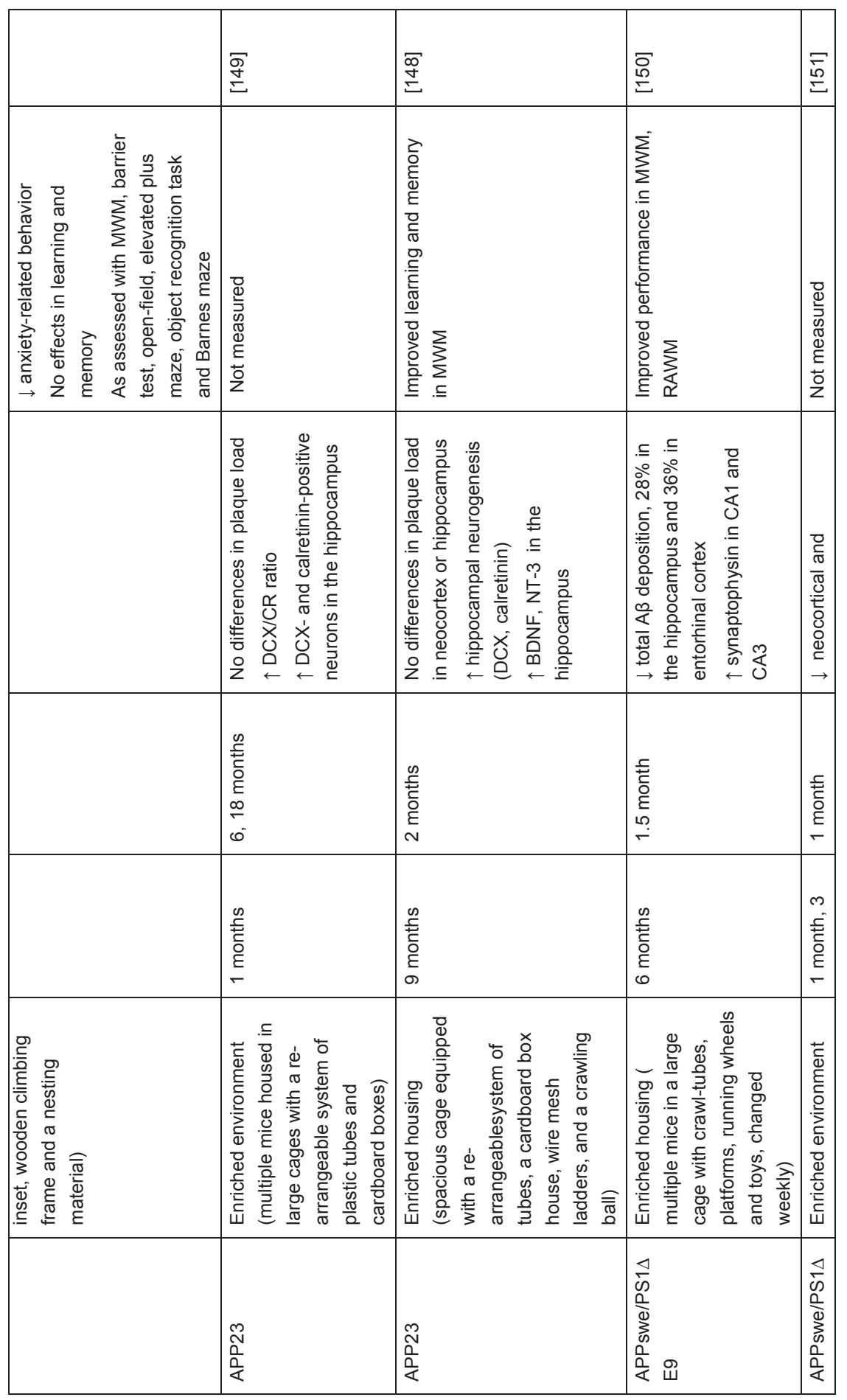




\begin{tabular}{|c|c|c|c|c|}
\hline & $\begin{array}{l}\mathbb{N} \\
\stackrel{\sqrt{2}}{\leftrightarrows}\end{array}$ & 䓢 & 莡 & 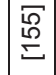 \\
\hline & 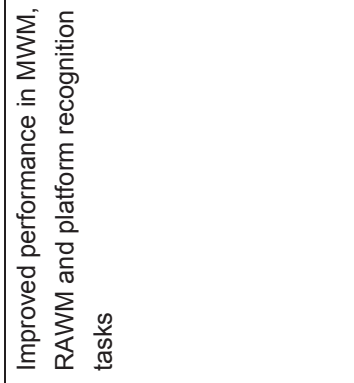 & 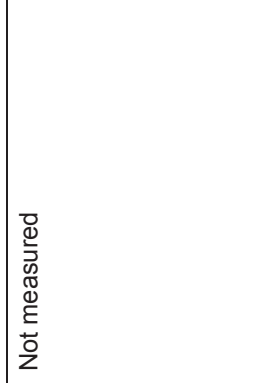 & 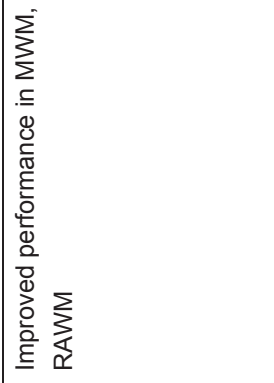 & 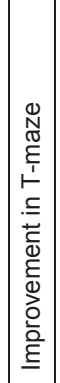 \\
\hline 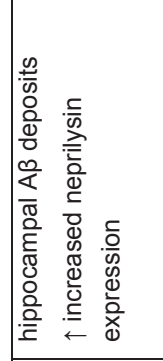 & 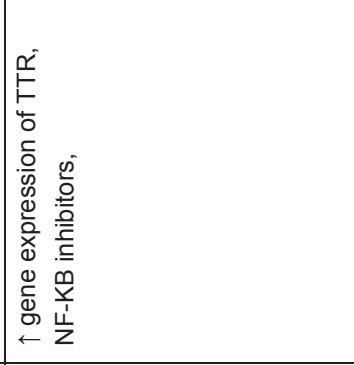 & 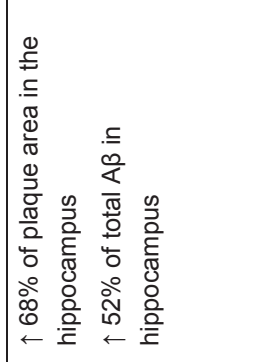 & 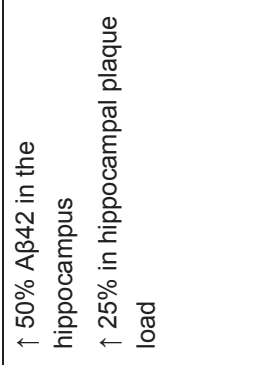 & 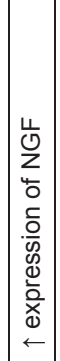 \\
\hline & 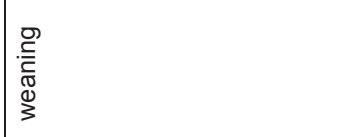 & 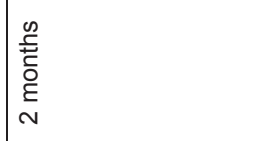 & 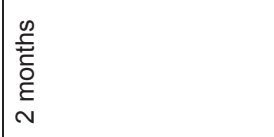 & 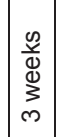 \\
\hline 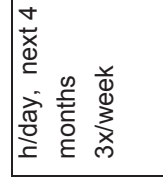 & 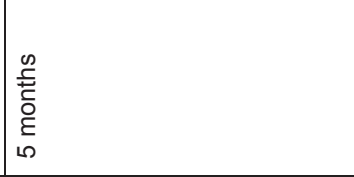 & 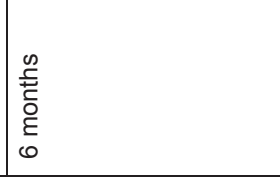 & 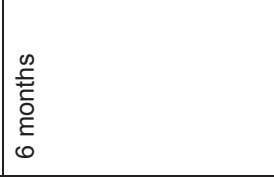 & 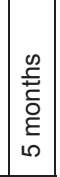 \\
\hline 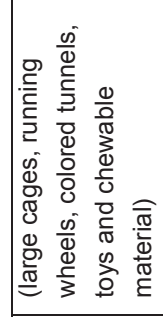 & 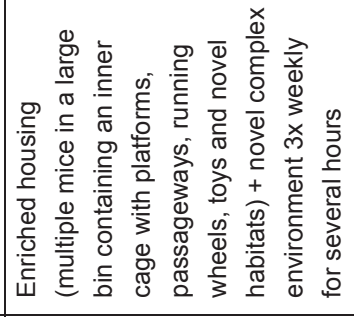 & 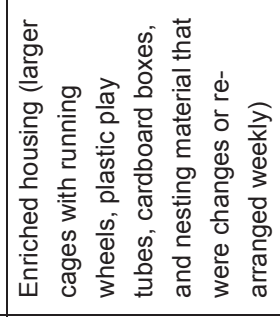 & 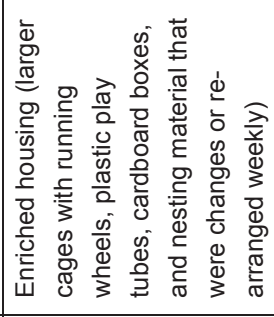 & 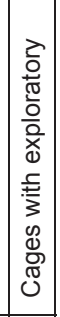 \\
\hline జ̊ & 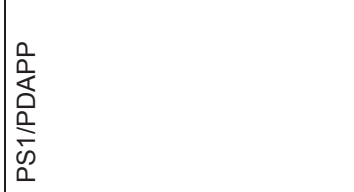 & 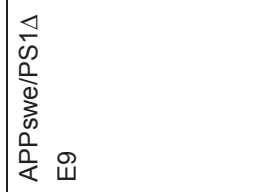 & 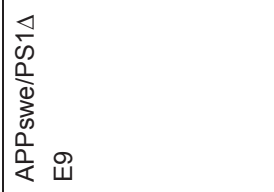 & 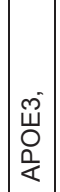 \\
\hline
\end{tabular}




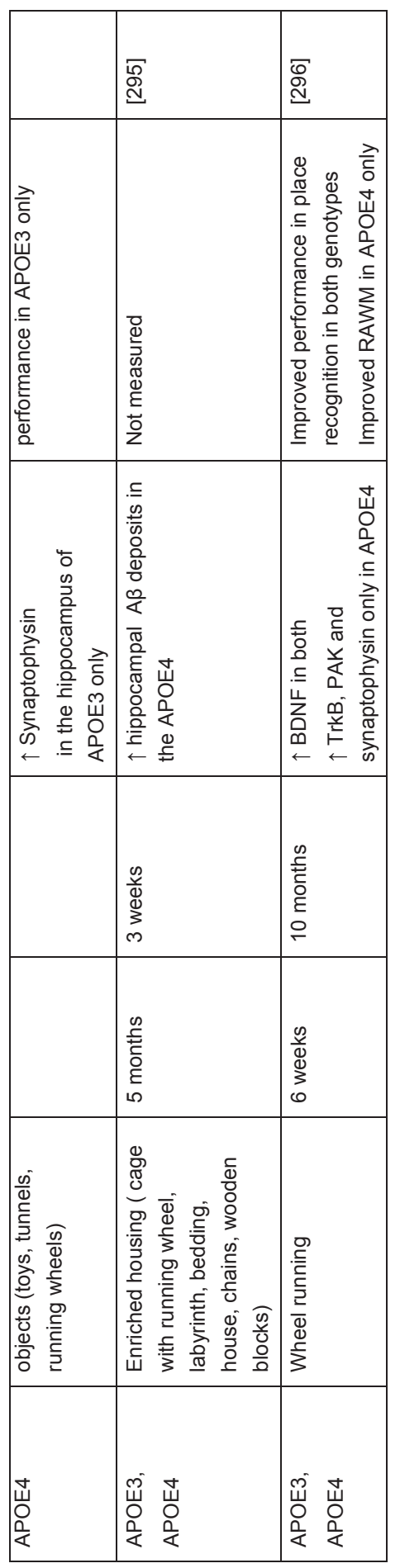




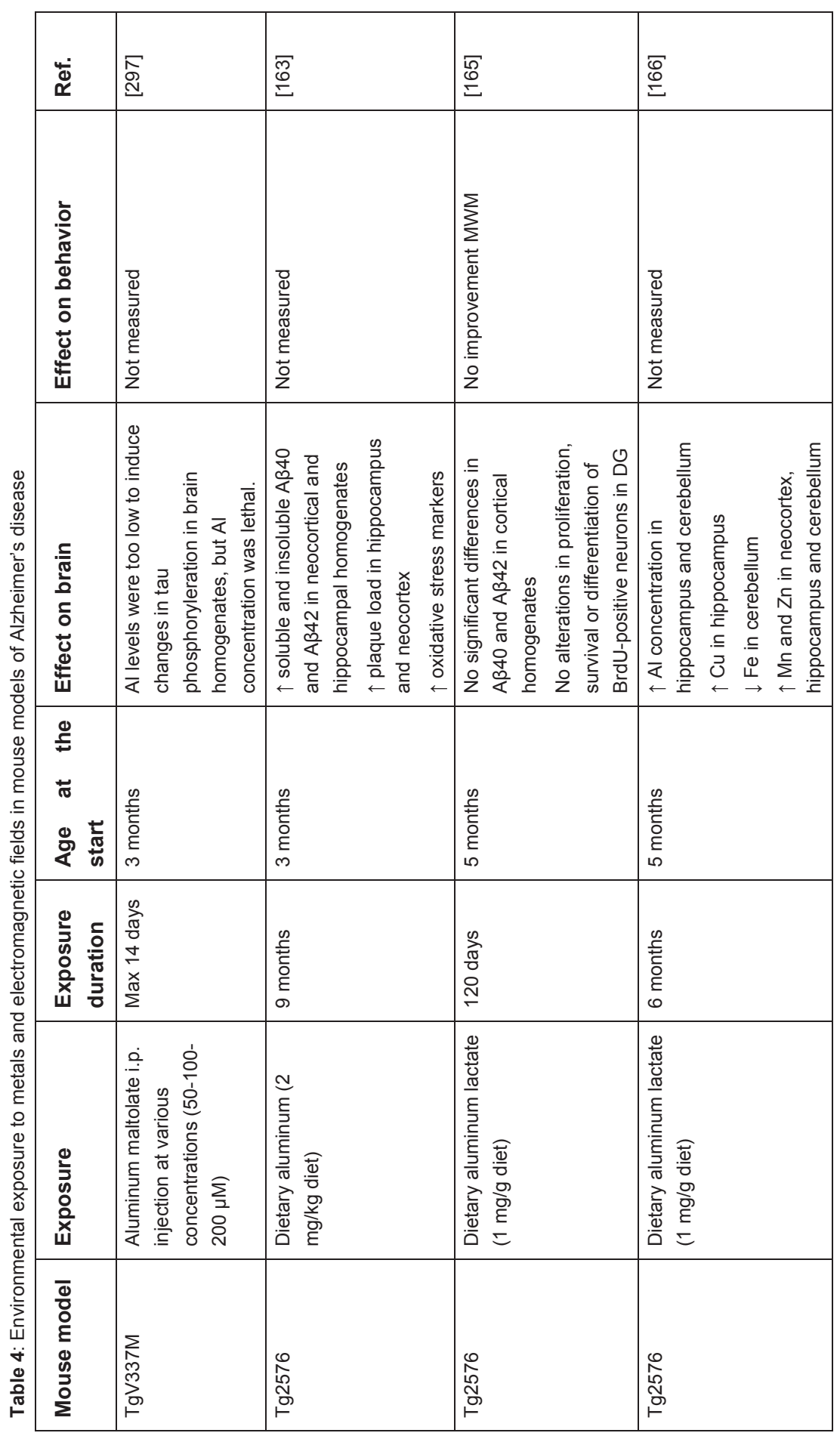




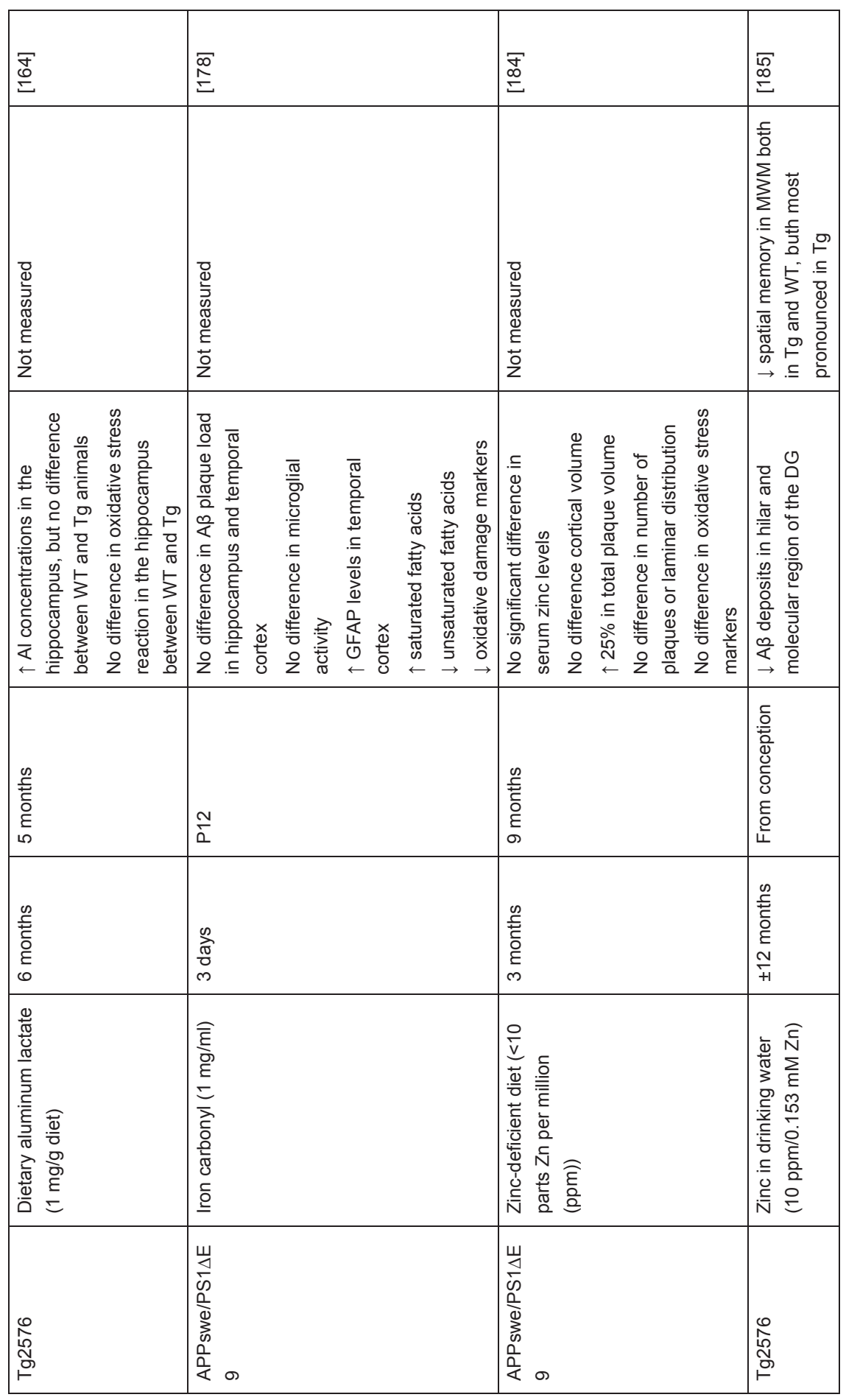




\begin{tabular}{|c|c|c|c|}
\hline $\begin{array}{l}\mathscr{\infty} \\
\stackrel{\infty}{\Xi}\end{array}$ & $\begin{array}{l}\mathscr{\Xi} \\
\stackrel{\infty}{\longleftarrow}\end{array}$ & 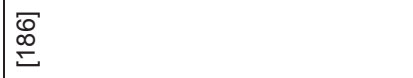 & 疍 \\
\hline 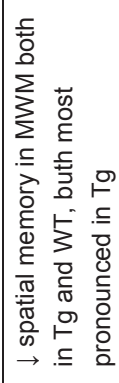 & 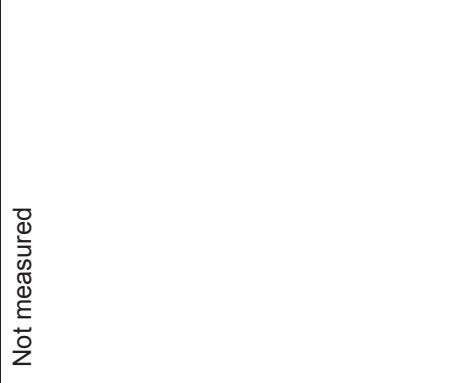 & 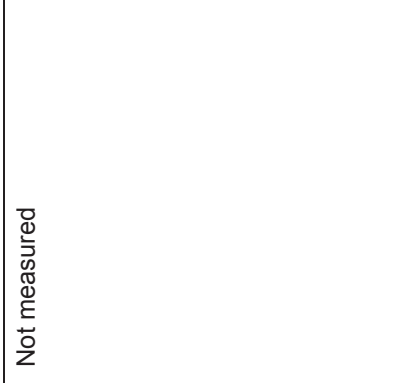 & 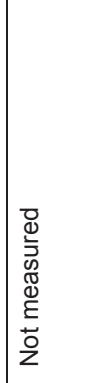 \\
\hline 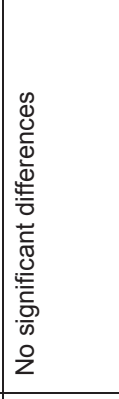 & 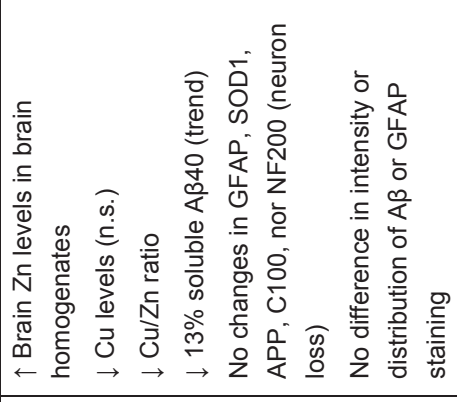 & 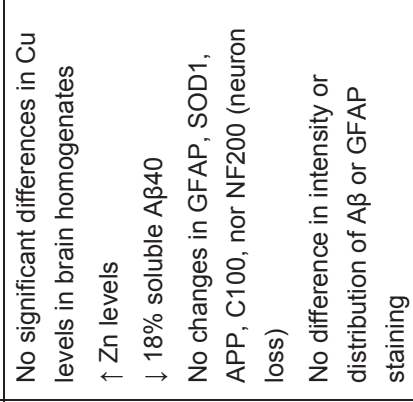 & 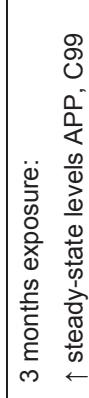 \\
\hline 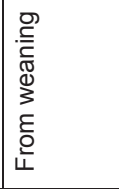 & 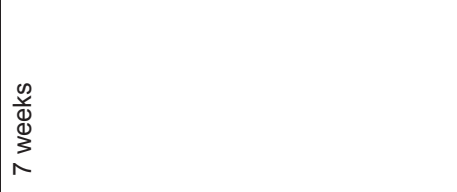 & 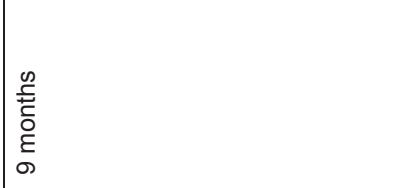 & 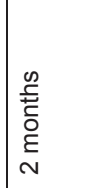 \\
\hline 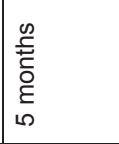 & 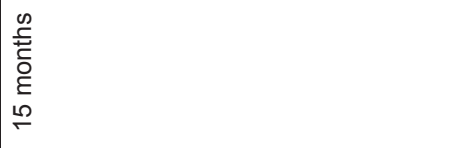 & 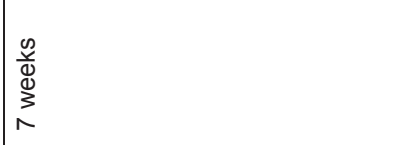 & 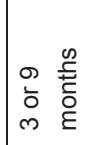 \\
\hline 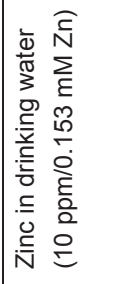 & 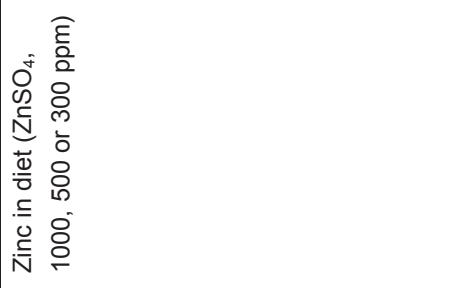 & 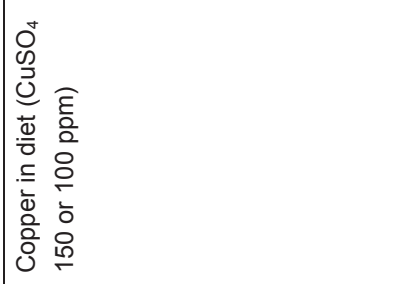 & 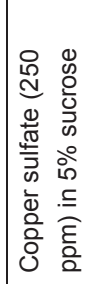 \\
\hline 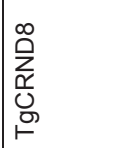 & 通 & 竞 & 吕 \\
\hline
\end{tabular}




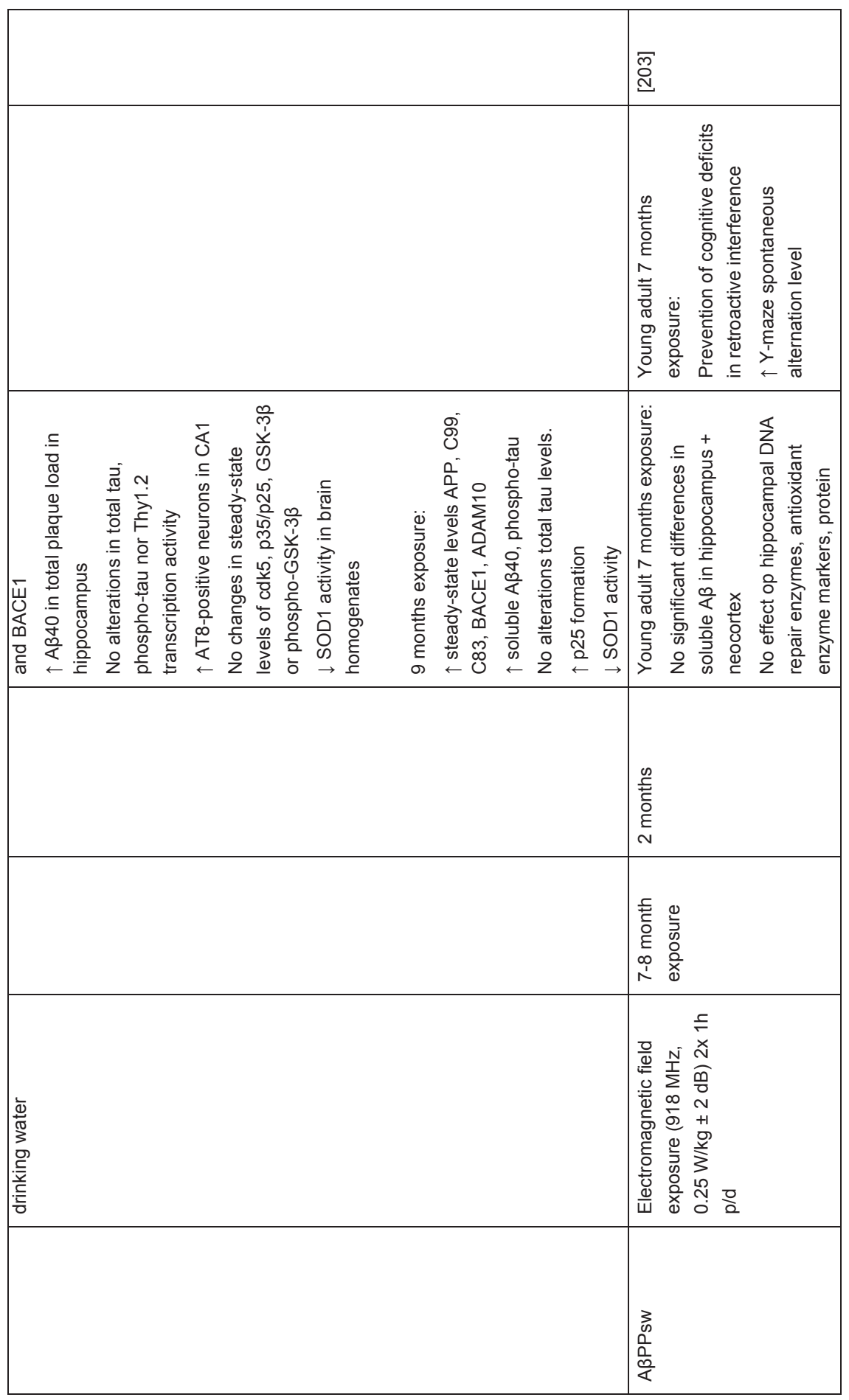


60|GxE in AD mouse models

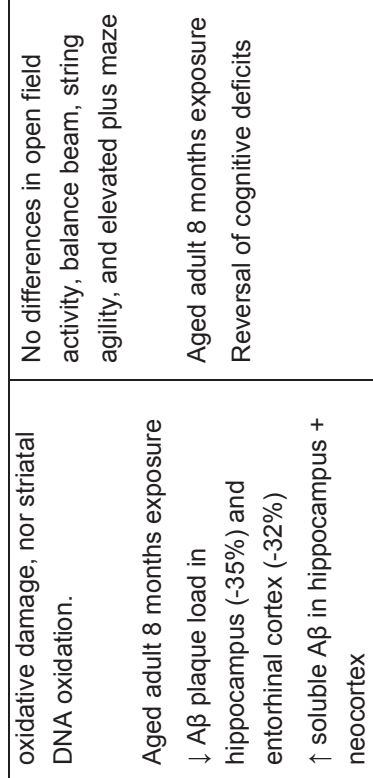

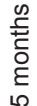




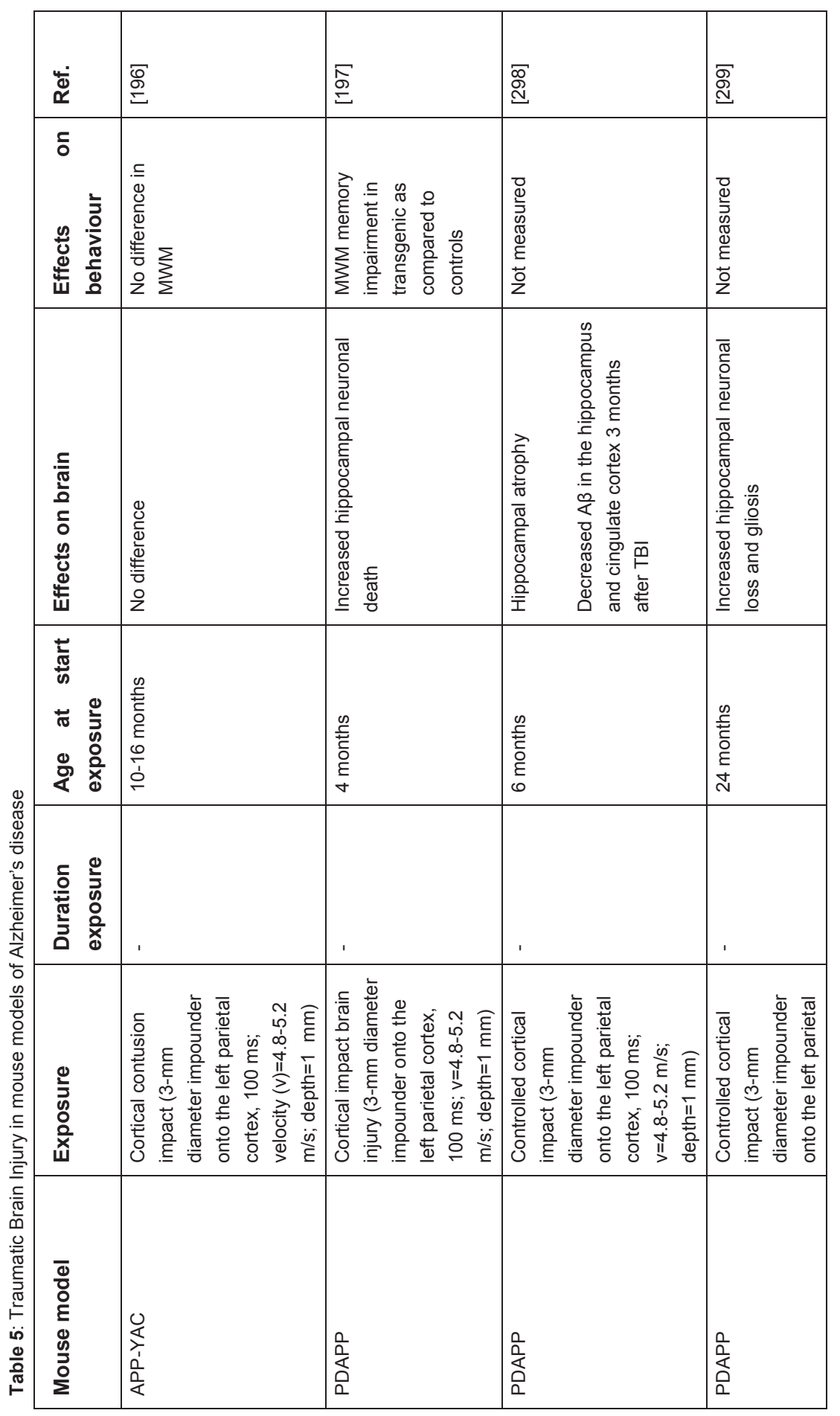




\begin{tabular}{|c|c|c|c|c|}
\hline & \multicolumn{2}{|l|}{ 氶 } & 灾 & 突 \\
\hline & \multicolumn{2}{|c|}{ 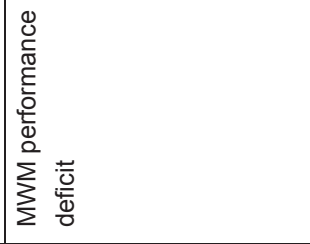 } & 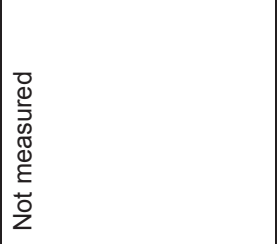 & 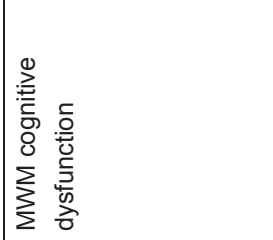 \\
\hline \multirow[t]{3}{*}{ 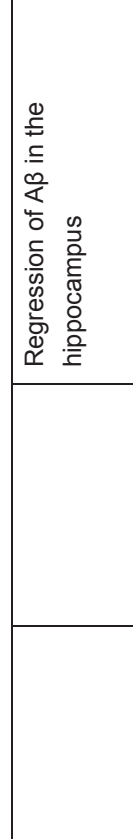 } & & 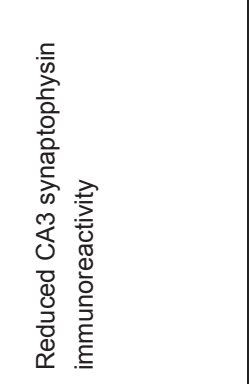 & 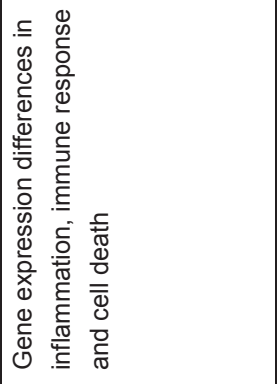 & 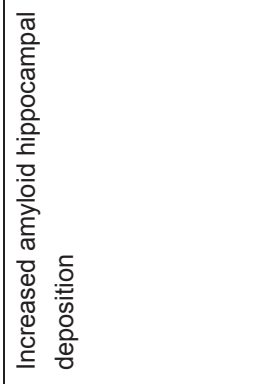 \\
\hline & \multicolumn{2}{|l|}{ 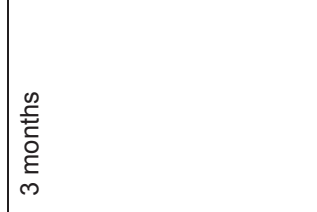 } & 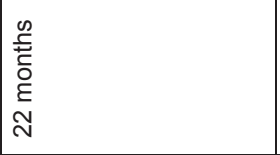 & 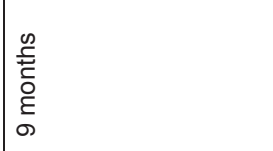 \\
\hline & ' & & . & 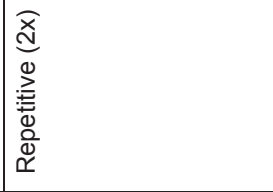 \\
\hline \multirow[t]{2}{*}{ 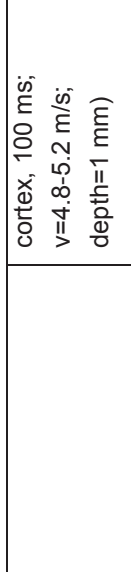 } & & 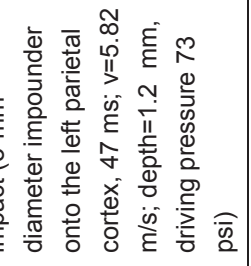 & 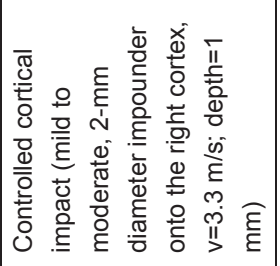 & 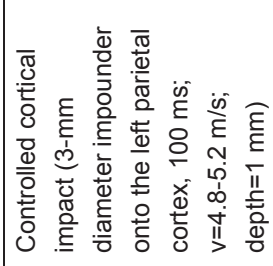 \\
\hline & \multicolumn{2}{|l|}{ 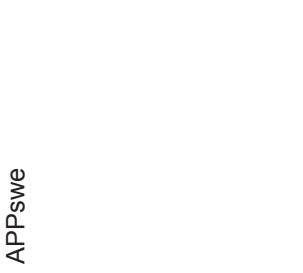 } & 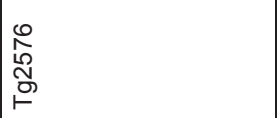 & 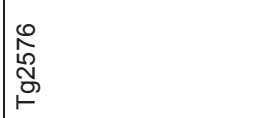 \\
\hline
\end{tabular}




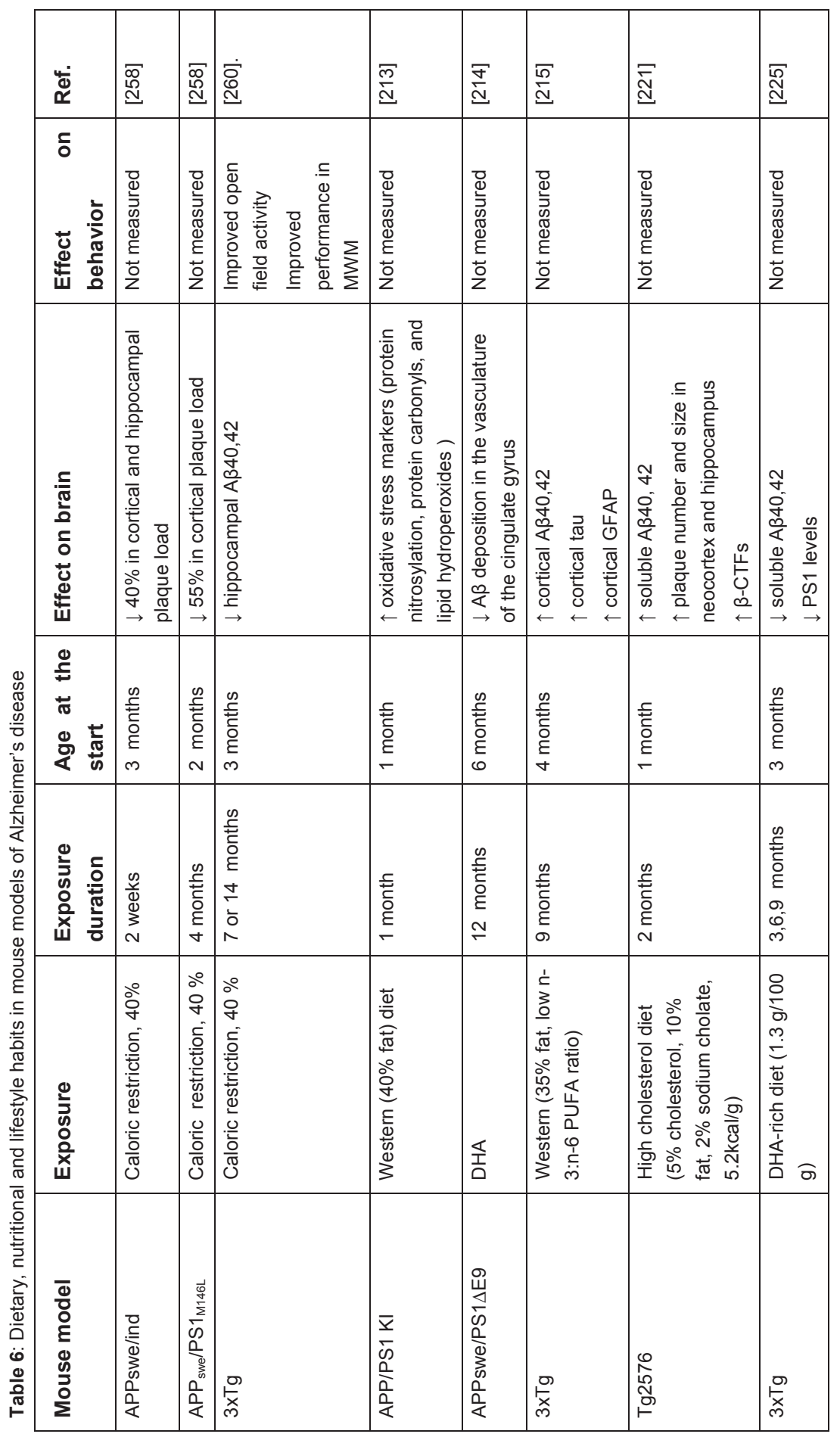




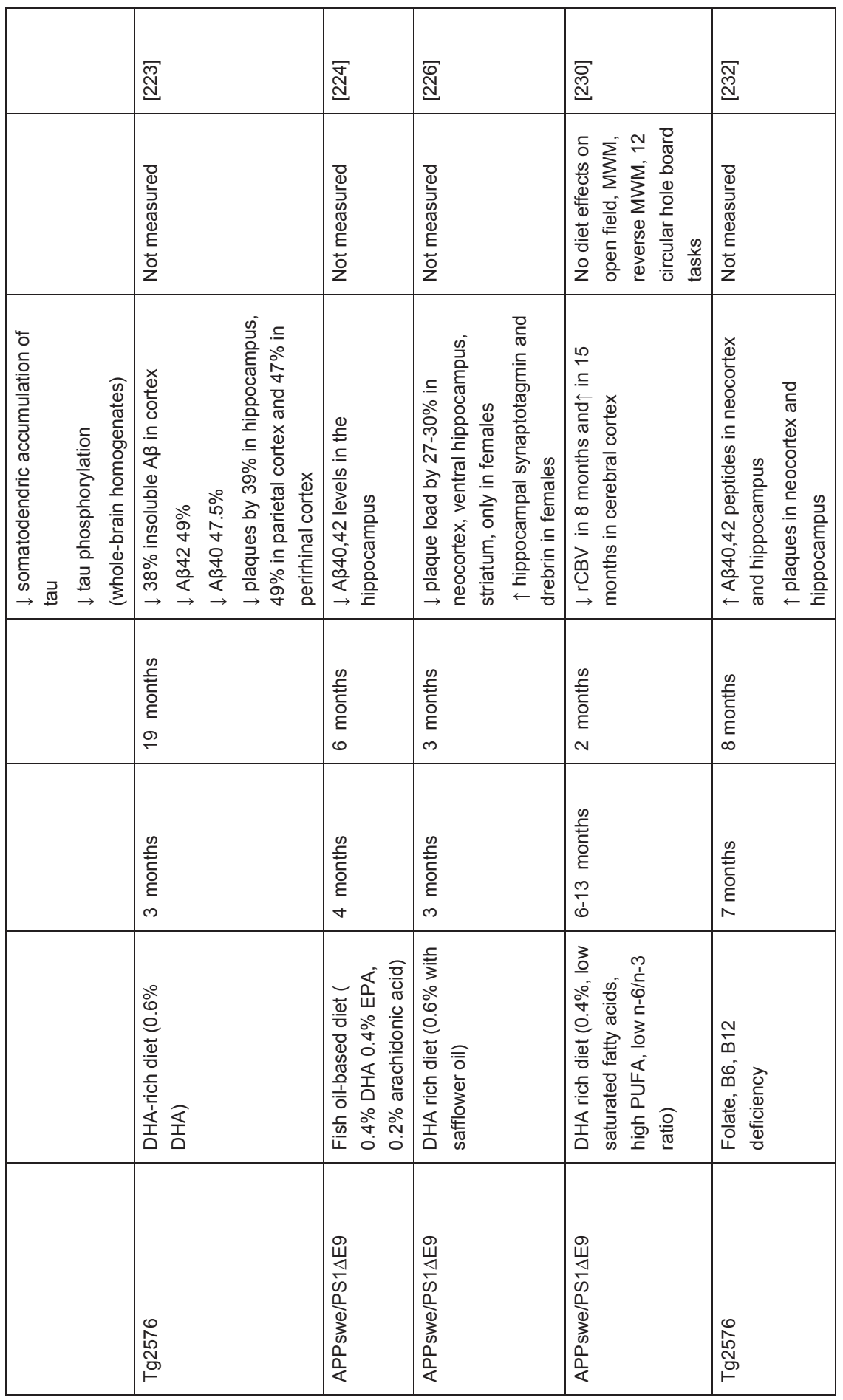




\begin{tabular}{|c|c|c|c|c|c|}
\hline 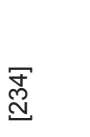 & 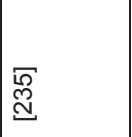 & $\begin{array}{l}\overline{\mathscr{D}} \\
\stackrel{\sim}{\mathrm{U}}\end{array}$ & 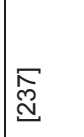 & 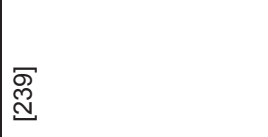 & 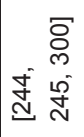 \\
\hline 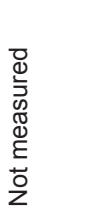 & 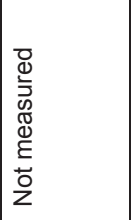 & 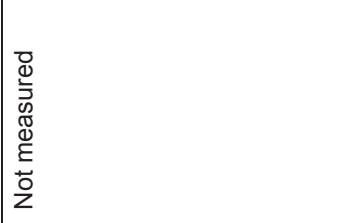 & 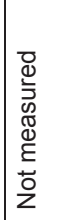 & 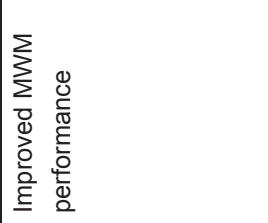 & 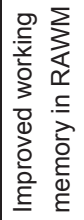 \\
\hline 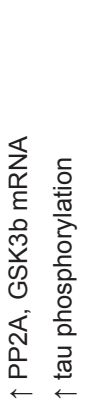 & 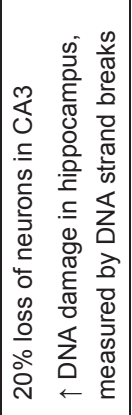 & 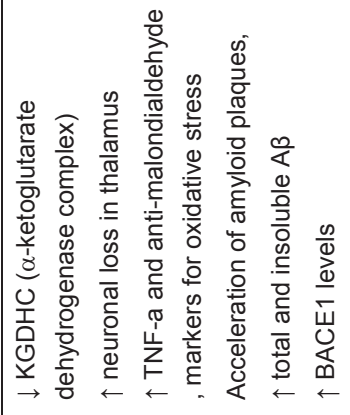 & 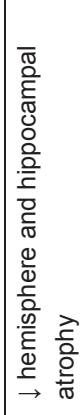 & 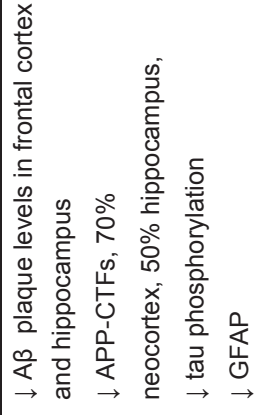 & 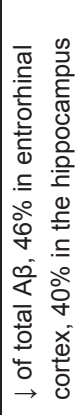 \\
\hline 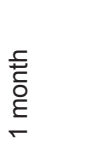 & 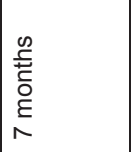 & 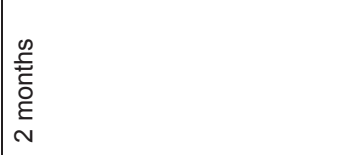 & 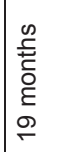 & 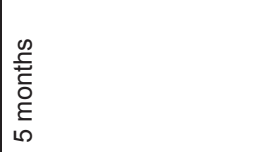 & 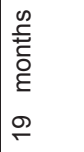 \\
\hline 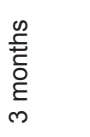 & 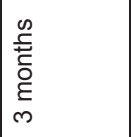 & 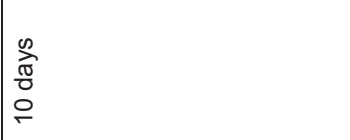 & 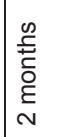 & 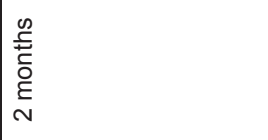 & 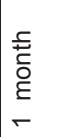 \\
\hline 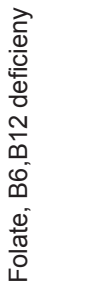 & 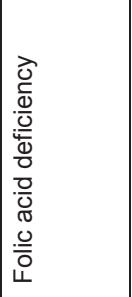 & 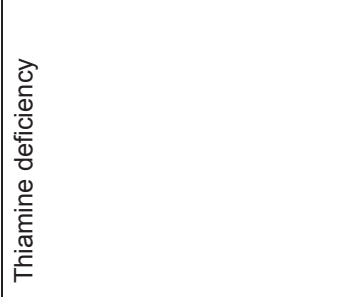 & 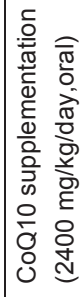 & 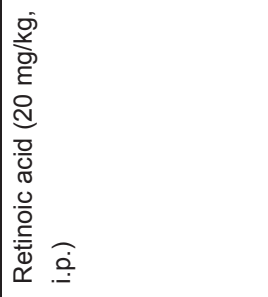 & 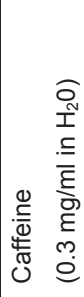 \\
\hline 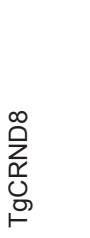 & 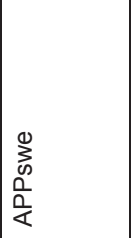 & 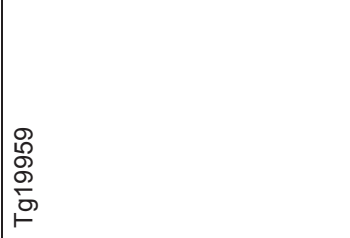 & 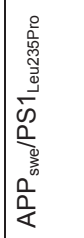 & 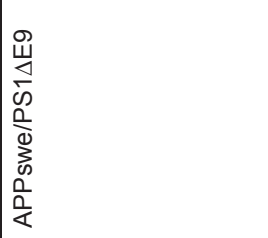 & 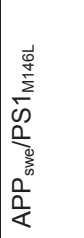 \\
\hline
\end{tabular}




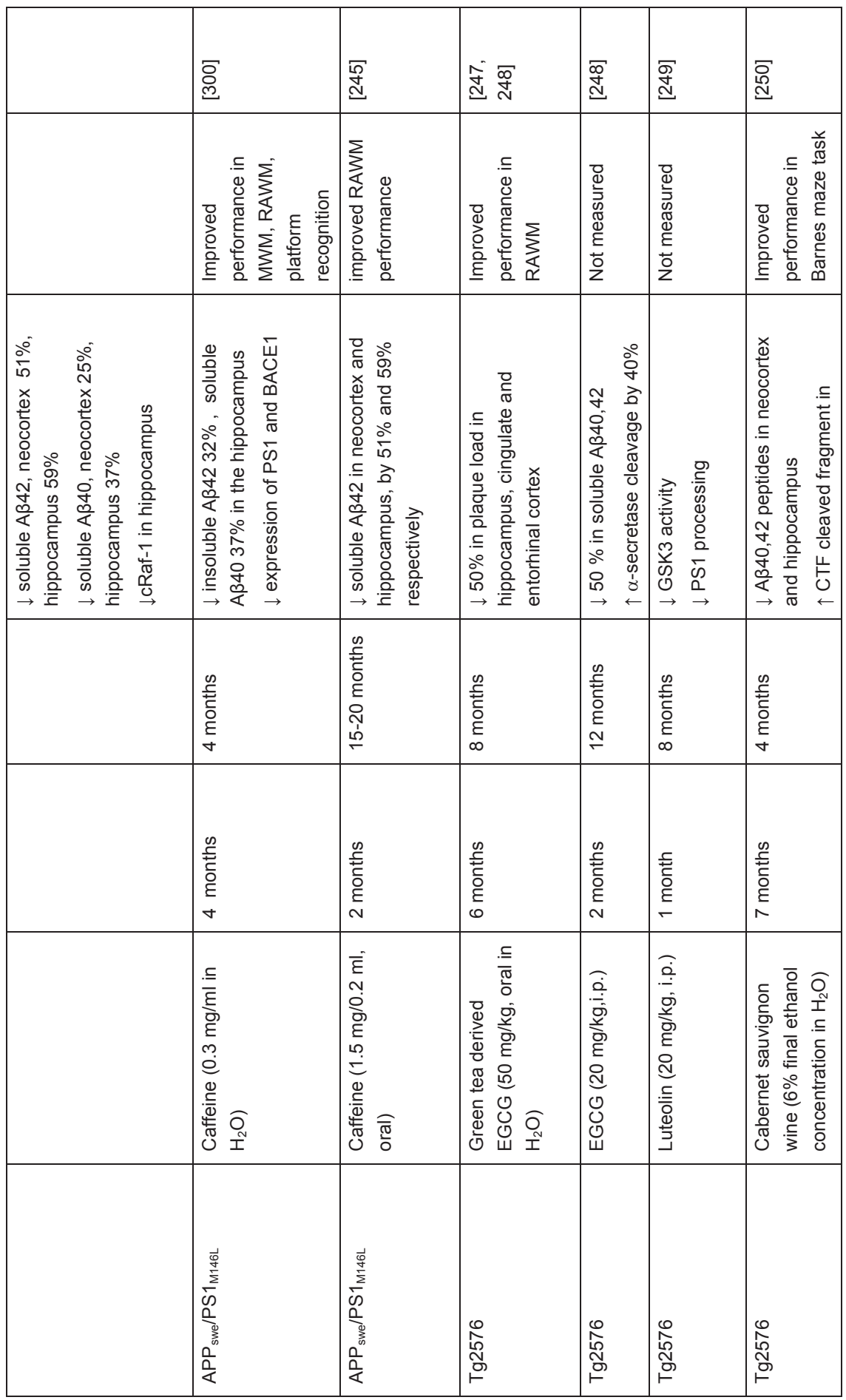




\begin{tabular}{|c|c|c|c|c|c|}
\hline & 点 & 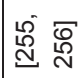 & 号 & 胥 & 㤟 \\
\hline & 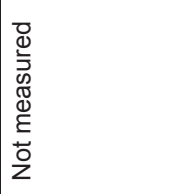 & 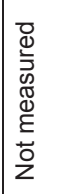 & 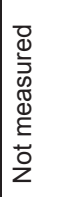 & 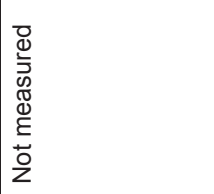 & 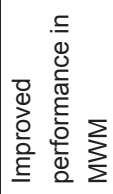 \\
\hline \multirow[t]{5}{*}{ 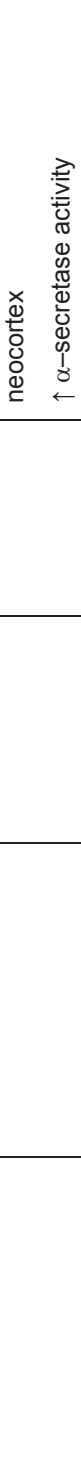 } & 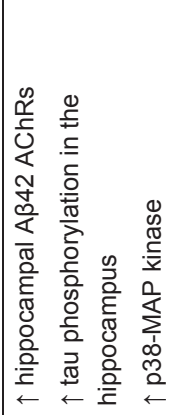 & 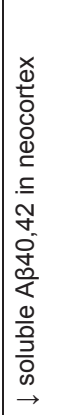 & 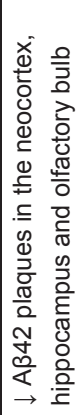 & 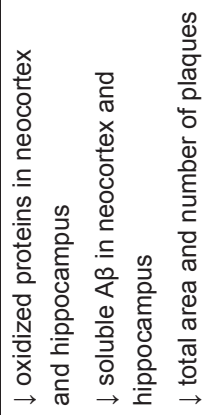 & 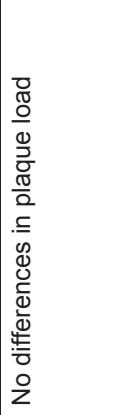 \\
\hline & 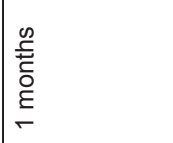 & 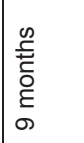 & 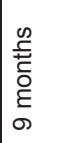 & 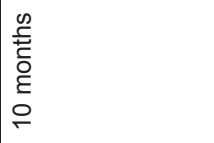 & 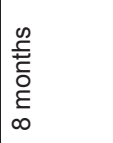 \\
\hline & 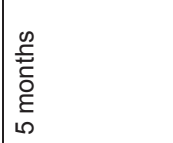 & 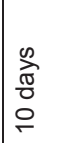 & 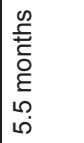 & 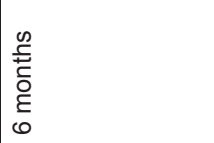 & 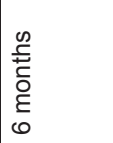 \\
\hline & 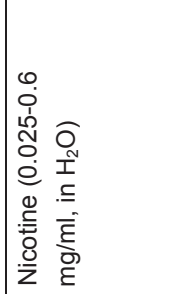 & 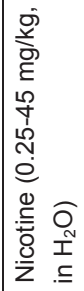 & 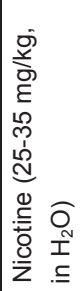 & 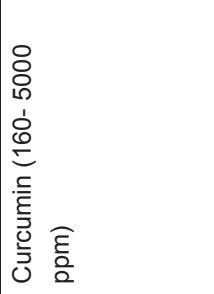 & 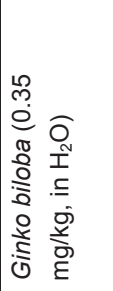 \\
\hline & 吕 & 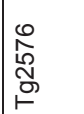 & $\begin{array}{l}0 \\
\stackrel{0}{2} \\
\text { J } \\
⺊\end{array}$ & 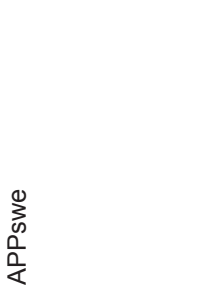 & 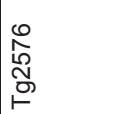 \\
\hline
\end{tabular}





\section{Chapter 3}

Major depression, cognitive dysfunction and Alzheimer's disease: is there a link?

Annerieke SR Sierksma, Daniel LA van den Hove, Harry WM Steinbusch, Jos Prickaerts

European Journal of Pharmacology (2010), 626(1), p.72-82 


\section{Abstract}

Major depression (MD) is a severe mental disorder characterized by alterations in mood and cognition, with disease severity correlating inversely with cognition scores. Neuropathology can be found abundantly in the limbic system, which is thought to regulate affect, attention and memory. Hypothalamic-pituitary-adrenal (HPA) axis overdrive, as well as decreased serotonin $(5-\mathrm{HT})$ concentrations, has often been implicated in the pathogenesis of this illness. Interestingly, there is substantial interaction between these two systems, with receptors of one system influencing the function of the other. This results in impaired neural networks, which give rise to the wide range of depressive symptoms. Recently, it has been implied that MD could serve as a risk factor for developing Alzheimer's disease (AD), with patients suffering from lifetime depression having a twofold higher chance of developing $A D$ and exhibiting more AD-related neuropathology. Modifications in the HPA-axis and the serotonergic system may contribute to the development of cognitive decline and eventually $A D$. These two systems may therefore be involved in the pathogenesis of both illnesses and could provide a link between $M D$ and $A D$. Obtaining more knowledge on their interactive role in the relation between MD and $A D$ may eventually aid in the development of more effective treatment strategies. 


\section{Introduction}

Major depression (MD) is the most common psychiatric disorder, with almost 1 in 5 people experiencing a depressive episode during their lifetime [301]. It is the fourth leading cause of global disease and disability burden and yearly 33.4 million people in Europe are suffering from this illness [302]. Besides its affective symptoms, such as anhedonia, depressed mood, feelings of worthlessness or guilt and recurrent thoughts of death or suicide, the patient often exhibits symptoms like appetite/weight disturbance, fatigue, and difficulties concentrating. These symptoms could be indicative of neuroendocrine and/or neuroanatomical changes [7]. Apart from the well-known affective changes in patients with MD, many also exhibit a large range of cognitive deficits. Common cognitive dysfunctions include impairments in recollection memory, list learning, recall, verbal and visual memory, executive function, attention and verbal fluency [303-308]. Severity of the depressive complaints can be correlated with cognition scores [309, 310].

It has become apparent that underlying systemic alterations can dramatically influence our affect and cognitive abilities. For example, alterations in the hypothalamic-pituitary (HPA) axis, as can be seen in patients with Cushing's syndrome, cause severe depressive complaints as well as many neuroendocrine changes also seen in MD [311, 312]. Likewise, the serotonergic system has been implied in the pathogenesis of MD, and antidepressants targeting this system have been shown to be very effective.

This review will discuss how the HPA-axis and the serotonergic system can influence the cognitive decline seen in MD patients, both independently as well as interactively. In addition, it argues the possibilty that MD could serve as a risk factor for Alzheimer's disease (AD), which is renowned for its progressive cognitive deterioration. The role of the HPA-axis and the serotonergic system in the development of cognitive decline in $A D$ is examined as well as the relation between MD and $A D$.

\section{Neuropathology in MD}

The wide range of symptoms in MD suggests that many different brain regions are involved in its etiology. Particularly the limbic system has received much attention, since this system is thought to govern our affective behaviour and is involved in learning and memory processes. Its main structures include the hippocampus, amygdala and prefrontal cortex (PFC), all of which are highly interconnected.

The hippocampus is known as the primary memory structure, necessary for declarative memory, or remembering facts. The amygdala is thought to control emotional processing, and is particularly involved in fear and aggression. Due to the high level of reciprocal connections, the hippocampus and amygdala are thought to regulate memory by modulating attention and consolidation processes, as reviewed in [313, 314]. The PFC is thought to exert top-down control over all emotions, thoughts and actions [315]. Imaging studies have 
shown abnormalities in structure and function within these areas in MD patients, although inconsistent results have been reported. Results are often confounded by the age and gender of the subjects, the number of depressive episodes, use of medication, definition of region of interest etc. (see [316, 317]).

The hippocampus is one of the key structures in the brain for learning and memory processes and alterations in its structure and function have been unequivocally established in patients with MD. For example, MD has been associated with increased hippocampal glucose metabolism [318] as well as reductions in hippocampal volume [305, 308, 319-323]. Moreover, clinical variables such as disease duration and time spent untreated correlated negatively with hippocampal volume [306, 308, 319, 322-324]. In addition, the hippocampal volume reductions as seen in MD correlated significantly with cognitive dysfunction and memory decrements [305].

It remains uncertain whether smaller hippocampal volume could be a predisposition for developing $\mathrm{MD}$, or whether it is a consequence of the disease process. Volumetric reductions have been reported as early as the first depressive episode [325], although others are not able to replicate these results [306]. MacQueen and colleagues [306] found that recollection memory was comprised as early as the first episode. Reductions in hippocampal volume, however, were thought to develop early in the disease process but not until multiple episodes have occurred. Hippocampal atrophy is thought to be caused by a reduction in neuropil, since no cell loss has actually been reported in MD. A decrease in the size of pyramidal and granular neurons, however, has been reported [326].

Another limbic structure, the amygdala, is particularly important for the perception and memory of emotions. It has many reciprocal connections with the hippocampus [327, 328] and it is thought that memory is shaped by an orchestrated interplay between these structures (as reviewed in [329]). Due to the high level of interconnectivity between the amygdala and the hippocampus, it was assumed that MD would also result in aberrant amygdalar structure and function. However, some degree of controversy remains about changes in amygdalar volume in MD. Some report volumetric decreases, which inversely correlated with the number of depressive episodes or degree of hippocampal volume loss [308, 330], while others claim that current depression leads to an increased amygdala volume, which could be correlated to disease severity [331]. A recent meta-analysis concluded that unmedicated MD patients exhibit a significant decrease in amygdala volume, while medicated patients show an increase [316].

With respect to its function, it has been reported that MD patients have higher glucose metabolism in the amygdala, which was positively correlated with negative affect, stress plasma cortisol levels (indicative of neuroendocrine stress) and severity of current depressive complaints [332-334]. MD is also associated with a decreased cerebral blood flow in the amygdala, which was inversely correlated with the severity of the depressed mood [335]. 
The PFC is thought to regulate and fine-tune all cognitive functions. Based on its anatomical connectivity and functional differentiation, the PFC can be divided into several subregions, such as the orbitofrontal cortex, the ventromedial PFC and the dorsolateral PFC (as reviewed in [336] and [337]. The ventromedial PFC is thought to be involved in regulating affective behaviour, as can be seen by its projections to the hypothalamus and its dense reciprocal connections with the amygdala. In contrast, the dorsolateral PFC is associated more with cognitive or executive functions, since it is highly connected to the orbitofrontal cortex and the hippocampus [338, 339].

It might not be surprising that the structure and function of the PFC is also altered in MD patients. The ventromedial PFC and the orbitofrontal cortex exhibit hyperactivity, while the dorsolateral PFC is hypoactive and associated with more negative emotions [318, 335, 340, 341]. In addition, hyperactivity in the orbitofrontal cortex can be directly correlated with anxiety levels and overall cognitive functioning [335] and reductions in volume and neuronal size have been reported in MD patients in the same area [342].

The exact mechanism underlying the changes seen in structure and function of various parts of the limbic system in MD, remains to be elucidated. However, several mechanisms have been proposed, such as dysfunctions in the HPA-axis or the serotonergic system.

\section{MD and hypothalamus-pituitary-adrenal axis}

The HPA-axis regulates the stress response by secreting specific stress hormones, or glucocorticoids. This stress response prepares the body and the brain for an adequate reaction to the stressor. Apart from its peripheral effects, the HPA also impacts the central nervous system. The secretion of glucocorticoids is orchestrated by the paraventricular nucleus (PVN) of the hypothalamus. In response to stress, the parvocellular neurons located in this nucleus synthesize and secrete corticotrophin-releasing hormone $(\mathrm{CRH})$ into the pituitary portal system. This causes the anterior pituitary to release adrenocorticotropic hormone $(\mathrm{ACTH})$, which in turn stimulates the adrenal glands to produce glucocorticoids (cortisol in humans, corticosterone in rodents). The release of glucocorticoids results in a stress response in the body, thereby altering energy metabolism and immunity. The glucocorticoids regulate negative feedback to the hypothalamus and pituitary by diminishing the secretion of $\mathrm{CRH}$ and ACTH. The hippocampus also has an inhibitory effect on the HPAaxis (see [343]).

Levels of circulating glucocorticoids are monitored by two types of receptors: the mineralocorticoid receptor and the glucocorticoid receptor. The mineralocorticoid receptor is a high-affinity receptor and located almost exclusively in the hippocampus. Under physiological conditions, basal levels of circadian glucocorticoids almost completely occupy these mineralocorticoid receptors, thereby giving negative feedback to the HPA-axis. Under stress or during circadian peaks, the low-affinity glucocorticoid receptors become activated 
and exert their negative feedback [344]. glucocorticoid receptors are located throughout the brain, but are most dense in the hippocampus, cortex, thalamus, hypothalamic PVN and the dorsal raphe nucleus $[345,346]$.

\subsection{Functional and structural alterations of HPA axis in MD}

A substantial load of evidence has shown that the HPA-axis is dysregulated in MD. Indeed, multiple clinical studies have indicated that patients with MD show higher baseline levels of cortisol in plasma, serum, cerebrospinal fluid and urine [347-351]. In the dexamethasonesuppression test, designed to assess HPA-responsivity, MD patients are less able to downregulate the stress response, which is indicative of dysfunctional feedback mechanisms [350, 352]. In addition, ACTH levels are elevated at baseline and after dexamethasone administration [351, 353, 354], although these results were not always replicated [355]. The combined dexamethasone/CRH test, which pretreats a person with dexamethasone before administrating $\mathrm{CRH}$, was designed to increase sensitivity in measuring abnormal HPA-axis function [356]. This test also demonstrated a significantly increased cortisol release in depressed patients [352, 357, 358].

Higher levels of glucocorticoids are thought to occur downstream from excessive $\mathrm{CRH}$ secretion. It has in fact been demonstrated that MD patients have a pronounced elevation of $\mathrm{CRH}$ in their cerebrospinal fluid [359-361] and a higher expression of CRH mRNA in the PVN [362]. Interestingly, suicide victims showed a $23 \%$ decrease in $\mathrm{CRH}$ binding sites in the frontal cortex [363], which confirms the expected downregulation of $\mathrm{CRH}$ receptors in the face of $\mathrm{CRH}$ hypersecretion.

\subsection{The glucocorticoid cascade hypothesis}

The negative feedback loop in which high levels of glucocorticoids suppress the production of $\mathrm{CRH}$ and $\mathrm{ACTH}$ is an important mechanism. Dysfunctional feedback may cause excessive responsiveness of the HPA-axis, which can have detrimental effects on both the body and brain. This is one of the theories in the etiology of depression, where prolonged exposure to high levels of glucocorticoids, due to chronic stress, damages the hippocampus and thus results in a downregulation of glucocorticoid receptors. This disables proper negative feedback to the HPA-axis. The subsequent disinhibition of the hypothalamus will in turn result in more glucocorticoid production. Subsequently, more glucocorticoid receptors will be downregulated and eventually compromise hippocampal neurons and projections, ultimately resulting in a vicious cycle. All physical, affective and cognitive changes in MD could thus be regarded as a consequence of this dysfunctional HPA-axis. This theory is known as the 'glucocorticoid cascade hypothesis of MD' [364]. In addition to an indirect receptor downregulation due to damage, it has lately become clear that the glucocorticoid receptor is directly downregulated and decreased in function in depressed patients (as reviewed in [365]), which negatively impacts the negative feedback loop.

Evidence for this hypothesis has been provided by the effects of chronic corticosterone treatment, which was shown to lead to a downregulation of glucocorticoid receptors in the 
CA1 and CA3 area of the hippocampus and a reduction in glucocorticoid and large CA3 cells [111]. In addition, chronic unpredictable stress, causing high levels of circulating glucocorticoids, was implied to decrease the volume of the CA3 region, and led to dendritic atrophy in CA3 pyramidal neurons [108, 109]. Furthermore, it decreased neurogenesis and impaired long-term potentiation, a cellular substrate for memory [108, 112, 366, 367].

Since the integrity of the hippocampal network becomes comprised due to high levels of glucocorticoids, it is not surprising that its function is also altered. Elevated plasma cortisol levels have been related to the cognitive impairments seen in MD patients [368]. Acutely elevated cortisol levels in healthy volunteers also resulted in impairment in declarative memory and spatial thinking tasks [369]. Moreover, increased cortisol levels in healthy elderly correlated negatively with explicit and declarative memory and selective attention performance [370, 371].

Interestingly, all cognitive deficits that occurred as a consequence of increased glucocorticoids are highly specific for hippocampal or prefrontal dysfunction. The distribution of the glucocorticoid receptor is not restricted to the hippocampus, but can also be found in other parts of the limbic system [372, 373]. Indeed, acute glucocorticoid administration has also shown to impair spatial working memory, implying PFC dysfunction [374]. Likewise, glucocorticoids can also alter amygdalar influence memory [371, 375]. For example, memory for emotionally arousing stimuli was enhanced when cortisol was administered before acquisition [376, 377]. Similarly, administration of the corticosteroid synthesis inhibitor metyrapone blocked long-term declarative memory for emotionally arousing stimuli [378]. Thus, increased levels of glucocorticoids may also impair the functional integrity of these structures and exert a detrimental effect on the entire limbic regulation of cognition.

\section{MD and the serotonergic system}

Another system that has been implicated in the etiology of depression is the monoaminergic system. The 'monoamine hypothesis of depression' states that MD is caused by a decrease of monoamines in the brain, i.e. serotonin and noradrenaline. This theory was based on the discovery that drugs altering central noradrenaline and serotonin (5-hydroxytryptamine (5HT)) levels had antidepressive effects.

Particularly the serotonergic system has received much attention in light of depressive research. The neurotransmitter $5-\mathrm{HT}$ is synthesized from the essential amino acid Ltryptophan in the serotonergic neurons in the raphe nuclei, located in the brain stem. From here, $5-\mathrm{HT}$ is transported throughout the brain, with highest fiber densities in the hippocampus, hypothalamus and amygdala [379-381]. There are numerous types of 5-HT receptors, but the 5-HT1A, 5-HT2A and $5-\mathrm{HT} 2 \mathrm{C}$ receptor have been targeted mostly in MD and antidepressant research and are thought to be involved regulating both in depression, anxiety and cognition (see [382, 383]). By interacting with the other neurotransmitter systems, e.g. the cholinergic system, 5-HT can influence several memory and learning 
processes. In fact, 5-HT modulates many cellular and synaptic functions necessary for proper memory and learning, such as dendritic and synaptic outgrowth and neurogenesis (as reviewed in [384].

The 5-HT1A receptor is expressed both pre- and postsynaptically. It is most densely expressed in the raphe nucleus, the limbic system and the cortex. The 5-HT1A receptors in the dorsal raphe nucleus are mainly somatodendritic autoreceptors, thereby inhibiting the serotonergic neurons of the dorsal raphe nucleus. In the hippocampus they are mainly located postsynaptically, where they mainly inhibit the pyramidal neurons of the CA1 subregion of the hippocampus [379, 385-387]. Hippocampal activation of the 5-HT1A receptor is thought to be detrimental to learning abilities, most probably through its interaction with acetylcholine [388].

By using functional imaging, depressive patients were shown to have decreased binding of the $5 \mathrm{HT}-1 \mathrm{~A}$ receptor in the raphe nuclei and limbic system [389]. These results were confirmed by Sargent et al. [390], who also indicated reduced binding in frontal and temporal cortices. Messenger RNA levels of 5-HT1A receptor were also decreased in the hippocampus and dorsolateral PFC due to depression [391]. Brains of depressed suicide victims additionally showed decreased binding capacity of the 5-HT1A receptors in the raphe nuclei and hippocampus [392-394]. However, alterations in actual 5-HT1A receptors are still under discussion, since some have also reported increases in the raphe nuclei and the ventrolateral PFC of depressed suicides [395, 396].

The 5-HT2A receptor is located postsynaptically and most dense in the cortex and to a lesser extent in the limbic regions, while none are located in the raphe. It is implied to additionally regulate the release of other neurotransmitters [385, 387]. Of interest is the discovery that activation of the 5-HT2A receptor by agonist 8-OH-DPAT can desensitize the $5-\mathrm{HT} 1 \mathrm{~A}$ receptor in the hippocampus, while the number or the affinity of $5-\mathrm{HT} 1 \mathrm{~A}$ receptor sites stayed unchanged [397].

The $5-\mathrm{HT} 2 \mathrm{C}$ receptor is also a postsynaptic receptor, which can be found primarily in the cortex and to a lesser extent in the amygdala and the CA1 and DG regions of the hippocampus [387]. This receptor is said to be involved in long-term potentiation [398]. Alterations in 5-HT2 receptors in depressed patients are still a matter of debate. 5-HT2 receptor binding in blood platelets was reported to be elevated [399], as were the postsynaptic binding sites in the frontal cortex of suicide victims [400-402]. However, others report lower levels of 5 -HT2 receptor binding with reductions as high as $76 \%$ in the frontal cortex and other cortical regions of MD patients [403, 404].

Apart from the serotonergic receptor, there is also a $5-\mathrm{HT}$ transporter (5-HTT), which is located at the nerve terminal of the presynaptic neuron and is responsible for clearing $5-\mathrm{HT}$ from the synaptic cleft. It is expressed abundantly in the raphe nucleus and in lower concentrations in the limbic system. It is the main site of action for selective 5-HT reuptake inhibitors (SSRIs). Post-mortem analysis of depressed suicide victims confirmed reduced mRNA levels of the 5-HTT, although the expression per neuron was upregulated [392] as well as reduced transporter binding in the PFC, particularly in the ventrolateral PFC [395]. 
During the last decade it has been postulated that the antidepressant effects of MD medication is dependent on an increase of neurogenesis in the hippocampus [405], the latter in which neurotrophic factors such as brain-derived neurotrophic factor (BDNF) are thought to play a key role (see $[406,407])$. There is a high degree of interaction between BDNF and the serotonergic system. For instance, serotonergic sprouting can be promoted by BDNF [408], and BDNF transcription can be generated by activation of the 5-HT receptors via cAMP-response-element-binding protein (CREB) [407]. Interestingly, reduced BDNF protein levels have been found in the hippocampus and PFC in the post-mortem brains of suicide victims [409]. In rats, chronic stress decreased the expression of mRNA BDNF [410] and BDNF protein [411] in the hippocampus. Moreover, having a single nucleotide polymorphism in the coding exon of BDNF i.e. carrying the methione (Met)-BDNF allele, is associated with depression and significantly reduced hippocampal volumes in depressed patients [412, 413]. Of therapeutic interest in the fact that in preclinical studies antidepressant treatment has shown to significantly improve BDNF levels (reviewed in [406]).

\subsection{Genetic variations of the 5HTT increase the risk for MD}

Over the past decade, more attention has been given to the possibility of genetic contributions to the etiology of MD. Even though no pure genetic cause for MD has been found to date, there have been indications for a genetic vulnerability. More specifically, a polymorphism was found in the regulatory region of the 5-HTT protein, also named the 5HTT-linked polymorphic region (5-HTTLPR) [414]. The short and long alleles in the 5HTTLPR have been shown to alter 5-HTT transcription. The short variant of the polymorphism reduces the expression of the $5 \mathrm{HT}$ transporter, while the long variant nearly doubles it.

The 5-HTTLPR polymorphism has been associated with both anxiety and depressive disorders $[414,415]$. In fact, experiencing a stressful life event in combination with having a short allele, predicted the development of MD and increased the severity of depressive symptoms and suicidal thoughts or attempts [416]. In addition, being homozygous for the short allele decreases memory performance in elderly [417], and the interaction between MD diagnosis and the 5-HTTLPR polymorphism was associated with a decrease in hippocampal volume [418]. Furthermore, carrying the short allele induces hyperreactivity of the amygdala in response to fearful stimuli [419]. Canli and colleagues [420], on the other hand, suggest that the amygdala is not hyperreactive in the face of negative stimuli, but rather that it has a decreased reactivity to neutral stimuli. This implies that carrying the 5-HTTLPR short allele can greatly alter emotional regulation in general, rather than it being specific to negative emotions [420]. 


\section{Interaction between 5-HT and the HPA-axis}

Even though the HPA-axis and the serotonergic system have been implicated separately in the pathogenesis of $\mathrm{MD}$, many reciprocal connections exist between the two systems. Accordingly, alterations in the HPA-axis also have an effect on the serotonergic system and vice versa. On a structural level, it has been shown that $\mathrm{CRH}$ immunoreactivity can be found in the serotonergic raphe nuclei and many of the limbic structures [421, 422]. Actually, in depressed suicide men $\mathrm{CRH}$ immunoreactivity is increased with approximately $40 \%$ in the raphe nuclei of the midbrain [423]. On a functional level, it has been demonstrated in vitro that administrating $\mathrm{CRH}$ swiftly elevates the firing rates of serotonergic neurons in the dorsal raphe nucleus [424]. Similarly, acute stress activates the $\mathrm{CRH}$ receptors which resulted in an increased release of 5-HT from the central nucleus of the amygdala [425].

High levels of corticosterone, induced by chronic stress, decreased 5-HT1A receptor binding and mRNA levels in the hippocampus [394, 426], but substantially increased mRNA levels in the frontal cortex [427]. Depletion of glucocorticoids caused by adrenolectomy significantly increased 5-HT1A receptor density in the hippocampus and the raphe nuclei, which was restored by corticosterone replacement [428, 429]. Moreover, corticosteroids also seem to alter 5-HT1A transcription, since its mRNA levels were highly increased after adrenolectomy [430]. Adrenolectomy has further shown to decrease 5-HT turnover rate in the hippocampus [431, 432]. Further, the 5-HT precursor L-tryptophan was significantly diminished following dexamethasone administration [433, 434]. Alternatively, the 5-HT metabolite tryptophan hydroxylase activity was enhanced by high levels of CFR [435].

The serotonergic system is also able to influence the HPA-axis. Activation of the 5-HT1A and $5-\mathrm{HT} 2$ receptors has been shown to increase corticosterone levels in rats as well as in humans [436, 437]. However, administrating the 5-HT1A agonist ipsapirone in MD patients actually diminishes the ACTH and cortisol response, indicating ineffective interaction between the 5-HT system and the HPA axis [437]. Depleting the hypothalamus from 5-HT using a neurotoxin in fact heightened the dexamethasone suppression of the HPA-axis [438].

In the PVN serotonergic axons have been found to directly innervate $\mathrm{CRH}$ immunoreactive neurons [439, 440]. Moreover, 5-HT1A and 5-HT2A receptors are expressed and colocalized in these neurons, thereby having the ability to alter HPA output directly [441, 442]. This was shown by the infusion of the 5-HT1A agonist 8-OH-DPAT into the PVN which elevated ACTH release. The reaction could be completely blocked by the $5-\mathrm{HT} 1 \mathrm{~A}$ antagonist WAY100635 [443]. The inhibitory effect of the 5-HT1A receptor is regulated by the 5-HT2A receptor. Activating this receptor led to a desensitization of the $5-\mathrm{HT} 1 \mathrm{~A}$ receptor, resulting in a decreased ACTH secretion downstream [442].

Interestingly, it can be seen that activation of the $5-\mathrm{HT} 1 \mathrm{~A}$ receptor can have a dual effect depending on its location. In the CA1 region of the hippocampus both the 5-HT1A receptor and the glucocorticoid receptor can be found abundantly [344, 385]. Moreover, it 
appears that reducing 5-HT levels in the hippocampus by using the neurotoxin 5,7dihydroxytryptamine, also significantly reduces glucocortiocoid mRNA expression levels in the dentate gyrus, CA1 and CA2 [444]. Activation of the 5-HT1A receptor hyperpolarizes the CA1 neurons [445], and can thereby exert a direct effect on the negative feedback from the hippocampus to the HPA-axis. It should be noted that the mineralocorticoid receptor is able to attenuate this 5-HT1A induced hyperpolarization, whereas the glucocorticoid receptor is not [446]. Due to the chronically elevated levels of glucocorticoid, cortisol will preferably bind to the glucocorticoid receptor over the mineralocorticoid receptor [344], making it unlikely that the serotonergic inhibition of CA1 neurons will be lifted.

On the other hand, activation of the 5-HT1A receptor in the PVN directly inhibits the HPA-axis. However, alterations in 5-HT1A binding, as exhibited in MD patients, can modify the communication of the serotonergic system with the HPA axis.

\subsection{Genetic variations interacting with the HPA-axis}

Interestingly, it appears that polymorphisms associated with a higher chance of developing an MD episode, also influence the HPA axis. For example, being homozygous for the short allele of the 5-HTT gene, increases morning cortisol levels, and the interaction of these factors is associated with cognitive decline, hippocampal volume decreases and a higher vulnerability for subsequent depressive episodes [417, 447]. In addition carrying the short allele and having a family history of MD or a personal history of stressful life events, significantly increases cortisol response after being faced with a stressor [448, 449].

Further, it has been demonstrated that polymorphisms in the glucocorticoid receptor unfavorably alter HPA-axis functioning (as reviewed in [450]. If these polymorphisms are also combined with adverse experiences during childhood, the severity of depressive symptoms is enhanced [451].

Taken together, it is clear that there is high level of interaction between the glucocorticoid and the monoaminergic hypothesis of MD. Since both systems alter proper functioning of the limbic system, it is likely that they exacerbate each other to cause the cognitive and emotional disturbances that can be seen in MD.

\section{The link between MD and AD}

It has been demonstrated that there is a link between depressive complaints and the risk of cognitive decline during aging. More specifically, it appears that higher levels of depressive symptoms lead to a more rapid decline of global cognition [128, 305, 452-455]. In addition, specific traits such as negative affect and distress proneness seemed to correlate inversely with cognitive decline $[72,117]$.

Recently it has been postulated that MD could also serve as an independent risk factor for developing $A D[71,74,117,118,120,310,456-460]$, possibly by reducing the brains 'reserve capacity' [117]. In fact, according to a meta-analysis, having a lifetime of depressive 
episodes increases the chance of developing $A D$ by at least twofold [460]. Some found that this effect was influenced by early onset of depressive complaints [74], a history of depressive episodes $[310,461]$, or education, with only those with more years of education being affected [74].

Interestingly, $A D$ patients with a history of MD show increased numbers of neurofibrillary tangles and amyloid plaques, the neuropathological hallmarks of $A D$, in the hippocampus. When these patients were suffering from MD at the time the clinical AD diagnosis was made, the presence of these hallmarks increased even further [128]. In addition, MD patients have lower amyloid- $\beta(A \beta) 42$ plasma levels, which normally is a biomarker for the onset of $A D$ [126]. Furthermore, using 2-(1-\{6-[(2-[ $\left.{ }^{18} \mathrm{~F}\right]$ Fluoroethyl)(methyl)amino]-2-naphthyl\}ethylidene)malononitrile (FDDNP), a molecule that binds to amyloid plaques and neurofibrillary tangles, with positron emission tomography (PET) in MD patients indicated that more severe depressive complaints correlated with the presence of these hallmarks in the medial temporal lobe [127].

Even though these studies imply that MD could serve as a risk factor for AD, the precise mechanisms underlying this development remain uncertain. Possibly, the HPA-axis and serotonergic system could play a pivotal role in this respect.

\section{1. $A D$ and the HPA-axis}

It is important to realize that $A D$ patients exhibit chronically elevated cortisol and $A C T H$ levels and markedly higher levels of $\mathrm{CRH}$ mRNA levels in the hypothalamic PVN compared to healthy controls, indicative of disturbance in HPA-axis homeostasis [107, 362, 462]. In addition, higher glucocorticoid levels were associated with more rapid cognitive decline [107]. Elgh and colleagues [463] indicated that high levels of cortisol after a dexamethasone suppression test combined with lower hippocampal volumes significantly decreased verbal and spatial memory abilities in AD patients. There appears to be an interaction between HPA-axis dysfunction and the neuropathological hallmarks of AD. For example, elevated cortisol levels in AD patients are associated with alterations in $A \beta 42$ and phosphorylated tau concentrations in cerebrospinal fluid [462].

Similar results have been found in animal models of $A D$. Several mouse models of $A D$, i.e. Tg2576, 3xTg-AD and APPV717I-CT100, were subjected to chronic stress, which resulted in increased corticosterone levels, more reactive astrocytes, higher amyloid plaque deposition, higher levels of interstitial $A \beta 40$ and $A \beta 42$, and a down-regulation of the $A \beta$ degrading enzyme MMP-2 [78, 131, 132, 134, 136, 137]. In addition, increased levels of the glucocorticoid receptor and the $\mathrm{CRH}-1$ receptor were observed, as well as a decrease in dendritic arborization of cortical neurons and a reduction in BrdU-positive cells in the DG [131, 136]. Importantly, it appears that there was a strong positive correlation between the number of amyloid plaques and corticosterone levels as well as with the expression of glucocorticoid receptors in the hippocampus [131]. Moreover, chronic stress in AD mice resulted in memory deficits $[78,134]$. 
Rats with a history of chronic unpredictable stress also showed increases in the amyloid precursor protein (APP), the $\beta$-site APP-cleaving enzyme (BACE) and the C-terminal fragment 99 (C99), all favoring the production of neurotoxic $A \beta$ peptides, which was exacerbated by glucocorticoid treatment. On a behavioural level deficits in spatial memory and high levels of anxiety were observed under these conditions [464]. Increases in A 442 levels after glucocorticoid administration were also seen in macaque nonhuman primates (Kulstad et al., 2005).

On a cellular level, similar results were obtained. Administering glucocorticoids resulted in an elevated secretion of $A \beta 40$ and $A \beta 42$ peptides, their precursors APP and C99 and their cleaving enzyme BACE $[137,465]$. Glucocorticoids are able to make neurons more vulnerable for $A \beta$-induced neurotoxicity, by enhancing intracellular calcium levels, increasing oxidative stress, decreasing levels MMP-2 and by down-regulating nuclear NF-kB [136, 466, 467]. With regard to the formation of aberrant tau protein, it appears that both chronic and acute stress lead to an increase in tau phosphorylation [134, 136, 468]. This was also seen when tau-expressing cells are treated with glucocorticoids [465].

It is clear that increased levels of glucocorticoids increase the secretion of $A \beta$ peptides and the phosphorylation of tau. However, some propose that these alterations are mediated via the glucocorticoid receptor and the glucocorticoid-responsive element in the promoter region of APP and BACE. In this case, the effects on tau would happen downstream from the changes in $A \beta$ [137]. Yet again, others claim that the interaction between abnormal HPAaxis functioning and $A D$ neuropathology is mediated via the $C R H$ receptor $[132,136,468]$.

\section{2. $A D$ and the serotonergic system}

With regards to the serotonergic system, it has become apparent that is also becomes comprised due to the degenerative nature of $A D$. Post-mortem $A D$ brains show more neurofibrillary tangles in the dorsal raphe nucleus and a reduction in neuron number and large neuron density compared to healthy controls [469-472]. The behavioural changes that can be seen in AD patients, may be, at least in part, attributable to losses in the serotonergic system [473]. Moreover, the 5-HTTLPR polymorphism has also been proposed as a riskfactor for late-onset $A D[474,475]$.

A decrease was found in 5-HT1A and 5-HT2A receptor binding sites in AD post-mortem and living brains, specifically in frontal, temporal and the parietal lobes, the amygdala and the hippocampus, with losses up to fifty percent [476-485]. A reduction in 5-HT transporter binding sites in the frontal and temporal lobes as well as in the raphe nuclei, hippocampus and several subcortical structures was also found [486-490]. Neither disease severity, plaque load, nor cortical pyramidal neuron loss could be correlated to 5-HT1a receptor losses [473]. In contrast, 5-HT1A binding in the hippocampus was associated with progressive cognitive dysfunction. In addition, 5-HT1A binding could be negatively correlated with FDDNP binding in the hippocampus [491]. 5-HT2 binding in the cortex can be correlated with neurofibrillary tangle formation [483]. Moreover, decreases in 5-HT binding in 
the temporal cortex, was associated with levels of general cognition as measured by the Mini-Mental State Examination [486].

Besides the structural and functional changes in the serotonergic system of AD patients, a link exists between $5-\mathrm{HT}$ and the production of $A \beta$. Serotonin increases the release of nonamyloidogenic APP via $5-\mathrm{HT} 2 \mathrm{~A}$ and $2 \mathrm{C}$ receptors, thereby disfavoring the formation of neurotoxic $A \beta$ [492]. According to Lezoualc'h \& Robert [493], this effect was mediated via the 5-HT4 receptor, which is expressed highly in the limbic system [494]. In AD patients a reduction in 5-HT4 receptor binding sites in the hippocampus and the frontal cortex has been observed (Reynolds et al., 1995). Via these mechanisms a reduction in 5-HT levels could potentially alter the cleavage of APP facilitating A $\beta$ production. Both effects could be blocked by receptor-specific antagonists [492, 493].

Antidepressants can significantly alter $A \beta$ production as well. Treating the $3 x \operatorname{Tg} A D$ mouse model of $A D$ with the SSRI paroxetine decreased levels of extracellular $A \beta 40$, intraneuronal $A \beta$, and tau immunoreactivity in the hippocampus and amygdala. In addition, spatial memory significantly improved due to the antidepressant treatment [495]. Another SSRI citalopram also altered $A \beta$ production, by increasing the level of non-amyloidogenic APP by threefold [496].

\section{Discussion}

It has become apparent that many players may contribute to the cognitive decline seen in MD. The overactivation of the HPA-axis in response to chronic stress, the subsequent downregulation in glucocorticoid receptors and hippocampal atrophy can be viewed as a putative mechanism. On the other hand, alterations in 5-HT receptors, albeit through polymorphisms in its promoter region, have also been implicated in significant limbic changes, leading to a broader spectrum of MD-related dysfunctions. The interaction between both systems is of vital importance, and may not be forgotten when researching this area. By back-and-forth stimulation of each others receptors, detrimental alterations in one system can be exacerbated by the other.

A similar pattern can be expected in $A D$, given the fact that both systems are also comprised in this illness. The overlap in pathology with respect to the HPA-axis dysfunction and reduced $5-\mathrm{HT}$ receptor binding is quite apparent. Figure 1 provides a simplified summary of the described interactions between the 5-HT pathway, the HPA-axis, MD and $A D . M D$ is associated with a dysfunctional HPA-axis and serotonergic transmission, thereby attacking the integrity of the limbic system. As a consequence, the so-called reserve capacity of the brain may become compromised, which will manifest itself by cognitive and affective disturbances. This lack in reserve makes the brain less able to handle subsequent AD-related neurodegeneration like the formation of neurofibrillary tangles and amyloid plaques $[497,498]$. 
Normally, the 5-HT system protects neurons from $A \beta$ neurotoxicity, by favoring the release of non-amyloidogenic APP. However, MD-induced alterations in the serotonergic system lift this protection, thereby exacerbating $A D$ neuropathology. Excessive levels of glucocorticoids aggravate $A \beta$ and neurofibrillary tangle formation as well. Due to the presence of serotonergic receptors in the HPA-axis and the abundance of glucocorticoid receptor, mineralocorticoid receptor and $\mathrm{CRH}$ receptors in the 5 -HT system, a change in one system can have detrimental consequences for the other, leading to a vicious cycle.

However, as can be seen in the figure, AD can also induce a higher vulnerability to develop $M D$, via the same mechanisms. Indeed, depression is a common feature among $A D$ patients, with prevalence estimates ranging from 30-50\% [499]. The idea has been proposed that $M D$ is not a risk factor for $A D$, but merely an early symptom $[117,130]$. It is thought that, especially in highly educated people, the cognitive changes are too subtle to measure, due to higher cognitive reserve, while the neurodegenerative nature of $A D$ has already compromised the affective system enough to be noticed [117, 130].

Another link between $M D$ and $A D$ could also be reflected in the change in BDNF concentrations. Both MD and $A D$ patients exhibit decreased mRNA levels of BDNF in the hippocampus $[409,500]$. Since BDNF is a key molecule in regulating hippocampal plasticity, it is consequently of vital importance for hippocampal integrity and optimal functioning. Mouse models of $\mathrm{AD}$, such as the J20 and PS1M146V knock-in model, showed hippocampus-dependent learning and memory deficits, which could be correlated to the reduction in neurogenesis in the PS1M146V knock-in mouse model [501]. In addition, the J20 mouse showed synapse loss and aberrant cell signaling which could be reversed by BDNF infusion [502]. Improvement of cognitive and affective disabilities in MD after antidepressant treatment has also been attributed to increases in BDNF [406]. In this line, enhancing BDNF levels by administrating antidepressants could potentially prevent ADrelated cognitive impairment.

In conclusion, it can be postulated that the cognitive decline seen in depressed patients can, at least in part, be explained by alterations in the HPA-axis and serotonergic system. By rendering the brain more vulnerable for subsequent neurodegeneration and contributing to the formation of $A D$ specific hallmarks, MD may be a risk factor for developing $A D$. Opinions on this matter are still divided, and only future research can provide us with new insights. 


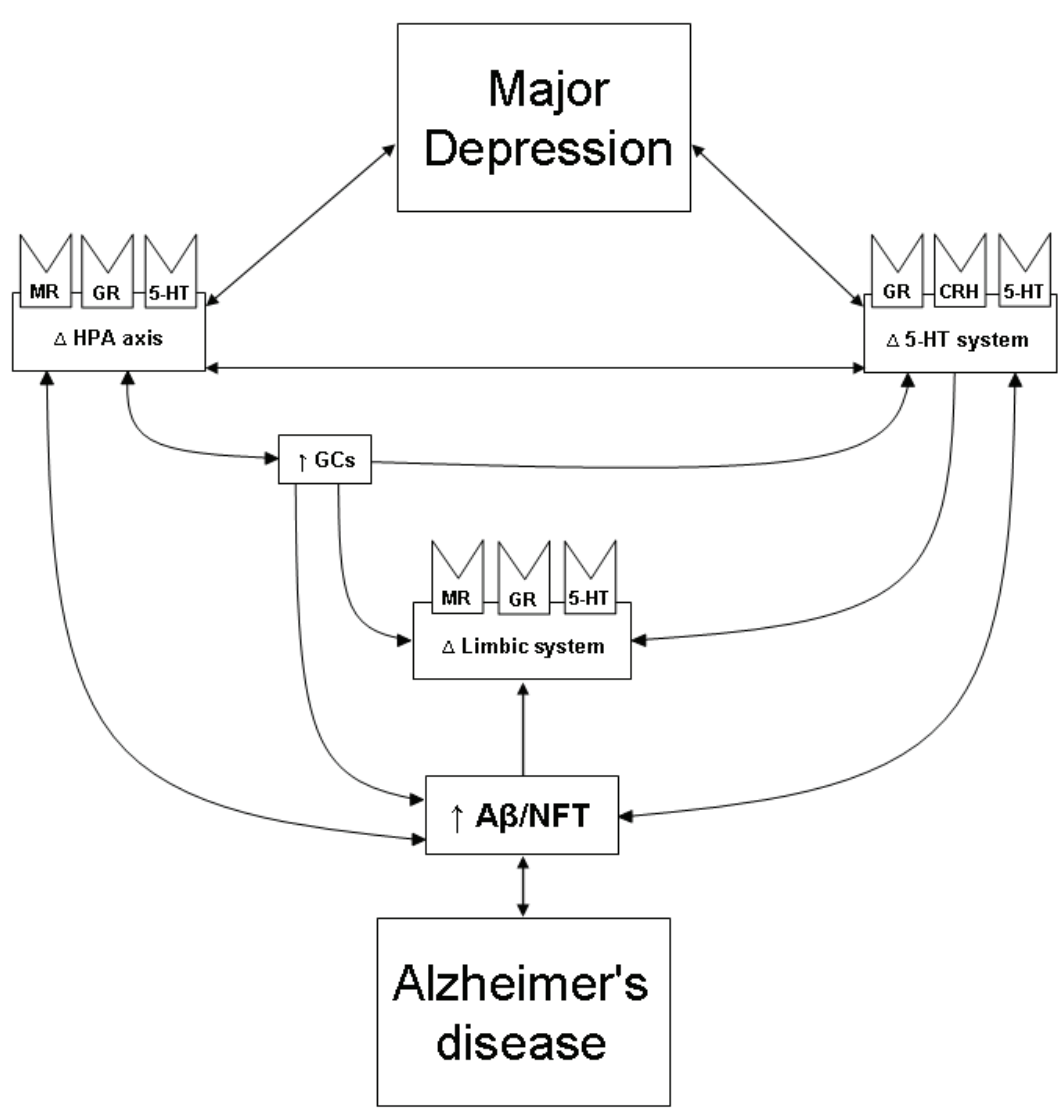

Figure 1: Schematic and simplified overview of the interactions between the HPA -axis and the serotonergic system. Major depression leads to alterations $(\Delta)$ in both systems, which are highly interconnected. Abnormal HPA-axis function results in an excessive release of glucocorticoids (GCs) which can alter mineralocorticoid, glucocorticoid and 5-HT receptor function and induce structural changes in the limbic system thus resulting in emotional and cognitive dysfunction. This renders the limbic system more vulnerable for neurodegeneration caused by Alzheimer's disease and further deteriorates emotional and cognitive fucntion. In addition both the 5-HT system and the glucocorticoids are able to influence production of amyloid- $\beta$ and neurofibrillary tangles. Abbreviations: $5-\mathrm{HT}$ : serotonin; $A \beta$ : Amyloid- $\beta$; $C R H$ : corticotrophin-releasing hormone; GCs: glucocorticoids; GR: glucocorticoid receptor; HPA: hypothalamus-pituitary-adrenal; MR: mineralocorticoid receptor; NFT: neurofibrillary tangles. 


\section{Acknowledgements}

The work of Dr. D. van den Hove and Dr. J. Prickaerts was partly funded by the European Commission Research Directorates, Research \& Technological Development Project, $6^{\text {th }}$ Framework Programme, LSHM-CT-2003-503474 (NEWMOOD). 


\section{Chapter 4}

Behavioral and neurobiological effects of prenatal stress exposure in male and female APPswe/PS1 $\triangle \mathrm{E} 9$ mice

Annerieke SR Sierksma, Jos Prickaerts, Leonidas Chouliaras, Somayeh Rostamian, Lore Delbroek, Bart PF Rutten, Harry WM Steinbusch, Daniel LA van den Hove

Neurobiology of Aging (2013), 34(1), p. 319-337 


\section{Abstract}

Epidemiological evidence implies a role for chronic stress and stress-related disorders in the etiopathogenesis of sporadic Alzheimer's disease (AD). Although chronic stress exposure during various stages of life has shown to exacerbate AD-related cognitive deficits and neuropathology in $A D$ mouse models, the role of stress exposure during the prenatal period on $A D$ development and progression remained to be investigated. The present study therefore explored the effects of prenatal maternal stress (PS) in both male and female APPswe/PS1 $1 \mathrm{E} 9$ mouse offspring in terms of cognition, affect and AD-related neuropathology. As prenatal perturbations are likely to mediate their effects via alterations in epigenetic regulation, changes in hippocampal DNA methyltransferase 3a (DNMT3a), 5methylcytosine $(5 \mathrm{mC})$ and 5-hydroxymethylcytosine $(5 \mathrm{hmC})$ levels were assessed as underlying mechanisms. Repetitive restraint stress during the first week of gestation exerted a sex-dependent effect, with male PS mice showing spatial memory deficits and a blunted hypothalamus-pituitary-adrenal axis response, while female PS mice showed improved spatial memory performance, increased depressive-like behavior, as well as a decrease in hippocampal plaque load. In addition, sex differences were observed among APPswe/PS1 $1 \mathrm{E} 9$ mice, independent of PS, i.e. female mice showed impaired spatial memory performance, higher hippocampal plaque load, altered amyloid precursor protein processing in the CA3 and lower DNMT3a immunoreactivity in the DG when compared to male mice of the same age. In conclusion, PS exposure impacts on the behavioral phenotype and neuropathology of APPswe/PS1 $\triangle \mathrm{E} 9$ mice. Moreover, given the remarkable sex differences observed, one should not overlook the impact of sex-specific responses to environmental exposures when investigating gene-environment interactions in AD. 


\section{Introduction}

Alzheimer's disease (AD) is the most common form of dementia, and characterized neuropathologically by extracellular plaques and intraneuronal neurofibrillary tangles. Its etiology remains largely unknown, although specific genetic mutations in the amyloid precursor protein (APP), presenilin 1 (PS1) and 2 (PS2) genes, resulting in aberrant production and cleavage of amyloid- $\beta(A \beta)$, have proven to be sufficient to cause $A D$ in its familial cases [503]. However, among sporadic cases, the etiopathogenesis remains unknown.

Epidemiological studies have indicated that environmental exposures, such as chronic stress and stress-related disorders, can impact on the progression of AD-related symptoms and pathologies [75, 128, 460]. However, investigating these effects in an epidemiological setting is challenging, given the large time span between exposure and clinical manifestation of $\mathrm{AD}$. In addition, the number, type and frequency of the exposures cannot be controlled for, making it difficult to unravel the contribution of each individual exposure. By using animal models, environmental exposure can be strictly controlled and AD-related behavior and neuropathology can be monitored throughout the disease progression (see [504]).

In this line, it has been demonstrated that $A D$ mouse models exposed to stress during various stages of life can alter AD-related symptoms and pathology. For example, isolation stress and chronic mild stress at weaning and early and late adulthood significantly decreased neurogenesis, increased $A \beta$ plaque formation and tau phosphorylation in the hippocampus and induced earlier manifestation of contextual memory deficits [76-78, 131, 505-507]. Similarly, immobilization or restraint stress in various AD mouse models during adulthood also resulted in elevated concentrations of $A \beta 40$ and $A \beta 42$, higher plaque deposition and increased tau phosphorylation in the hippocampus [76, 134-136, 508]. In addition, these mice displayed augmented neuronal degeneration with concomitant cognitive deficits [76, 78, 134-136]. Nonetheless, stress exposure during prenatal development has not been investigated yet in an $A D$ mouse model, even though it is well-documented that prenatal maternal stress (PS) exposure can contribute profoundly to an individual's predisposition for developing adult psychopathology [274, 509], has shown to negatively affect cognition and hippocampal functioning in various mouse models [510-513], and does so in a sex-specific manner [274, 510, 511, 514].

Stress exposure is likely to alter $A D$ development by modifying the effects of an individual's genetic predisposition [98, 515], for example, through epigenetic regulation of gene expression, i.e. via modifications in DNA methylation and chromatin remodeling [516, 517]. Interestingly, the prenatal period is characterized by high levels of epigenetic programming and imprinting, enabling perturbations to exert long-lasting effects. In this light, it was suggested that the origin of most diseases that are expressed later in life, such as $A D$, often find their origin in altered epigenetic regulation in response to environmental cues arising during critical periods of development, such as the pre- and neonatal period [518- 
521]. In addition, a 'two-hit' model of late-life aberrant expression (LEARn) was proposed [80], where a first hit resulting in altered epigenetic regulation, is followed by a second hit later in life. This latter could encompass additional environmental triggers or age-associated alterations in gene-expression levels, thereby giving rise to a diseased state. Thus, this model suggests that the epigenetic machinery serves as a central mechanism mediating the effect of PS on age-related neurodegeneration. Indeed, it has been shown in patients that $A D$ is associated with differential profiles of DNA methylation and histone acetylation, particularly of genes with a known involvement in $A \beta$ or tau processing (for an extensive overview see $[98,515])$.

Accordingly, the present study aims to explore whether the combination of genetic variations giving rise to early onset $A D$ and exposure to stress during prenatal life can alter behavioral and neuropathological phenotypes in mice. To that extent, APPswe/PS1 $1 \mathrm{E} 9$ mice, a model for $A D$, were exposed to $P S$ and the behavior, hypothalamic-pituitary-adrenal (HPA) axis and brains of 7-month-old male and female offspring mice were investigated. Behavioral and neuroendocrine phenotyping consisted of the object location task (OLT) and Y-maze to assess cognition, the elevated-zero maze (EZM) and forced swim test (FST) to measure alterations in affective behavior, and corticosterone concentrations in blood plasma before and after an acute stressor to explore HPA axis alterations. Neuropathological phenotyping was performed by determining hippocampal plaque load, intracellular $A \beta$ and synaptophysin-immunoreactive presynaptic bouton (SIPB) density. In addition, we assessed whether changes in the AD-related phenotype could be explained by alterations in global DNA methylation and hydroxymethylation levels in the hippocampus, i.e. DNA methyltransferase 3a (DNMT3a, a de novo methylase), 5-methylcytidine (5mC, a DNA transcription repressor) and 5-hydroxymethylcytidine $(5 \mathrm{hmC}$, a DNA demethylation intermediate) immunoreactivity, as prenatal exposures are likely to affect epigenetic regulation.

\section{Material and Methods}

\subsection{Breeding}

All animals were housed under a $12 \mathrm{~h}$ light/dark cycle (lights on at 7:00 A.M, unless specified otherwise) with an average temperature of $22^{\circ} \mathrm{C}$ and relative humidity of $42 \%$. Food and water was provided ad libitum throughout the study. All studies were executed according to protocols approved by the local Animal Ethical Committee of Maastricht University, Maastricht, The Netherlands (UM-DEC 2008-004) and met governmental guidelines. To obtain offspring, virgin female C57BL/6 mice (obtained from Charles River, L'Arbresle, France) were mated with male APPswe/PS1 $\triangle E 9$ mice (initially derived from Jackson Laboratory, strain \#005864, Bar Harbor, ME, USA) at 8-12 weeks of age. The latter express mouse/human chimeric APP695, harboring the Swedish K694M/N595L mutation, as well as 
the PS1 gene with a deletion of exon 9 (PS1 $\triangle \mathrm{E} 9)$ under the mouse prion protein promoter [47], and were backcrossed to a C57BL/6 background for at least 8 generations. This mouse model displays $A \beta$ plaques from about 4 months of age and shows the first hippocampal functional deficits from 7 months of age onwards [50-52]. Presence of a copulation plug was considered embryonic day 0 and, subsequently, the female was housed individually, given tissue for nest building, and assigned to a treatment group, i.e. PS or control.

\subsection{Prenatal stress paradigm}

PS exposure was carried out as described previously [510]. In brief, pregnant females assigned to the PS groups were subjected to chronic restraint stress by placing the mouse in a well-ventilated $5 \mathrm{~cm}$ diameter glass cylinder, filling the cylinder with water to a height of 1 $\mathrm{cm}$ and positioning them underneath a bright light. Restraint stress was performed 3 times per day (at approximately 8:00h; 12:00h; 16:00h), for 45 min per session from embryonic day 1-7, as it was demonstrated that early gestational stress (from embryonic day 1-7) has more impact on the behavioral phenotype of C57BL/6 offspring when compared to late gestational stress (from embryonic day 15-21) [511, 514]. After each restraint stress session, the animals were placed back into their home cage. Control mice were left undisturbed in their home cage. All female mice were monitored for weight alterations and given a clean cage at embryonic day 0,7 and 14 .

\subsection{Offspring}

At postnatal day 25 all animals were ear-punched and marked to enable permanent identification while harvesting tissue for genotyping. Genotyping was performed using PCR amplifications of genomic DNA. Two PCR reactions were performed: one for determining the presence of the APP mutation and the other for the presence of the PS1 mutation. Each reaction contained primers for the mouse prion protein promoter (sense: CCT CTT TGT GAC TAT GTG GAC TGA TGT; antisense: GTG GAT AAC CCC TCC CCC AGC CTA GAC C) and either primers for APP (sense: GAC TGA CCA CTC GAC CAG GTT CTG; antisense: CTT GTA AGT TGG ATT CTC ATA TCC) or PS1 (sense: AAT AGA GAA CGG CAG GAG CA; antisense: GCC ATG AGG GCA CTA ATC AT). The endogenous prion protein promoter produces a $750 \mathrm{bp}$ product, while transgene positive-samples produce bands at $450 \mathrm{bp}$ (APP) or $600 \mathrm{bp}$ (PS1). At postnatal day 30 ( \pm 1 day), pups were weaned and housed per 2 with same sex littermates. Four experimental groups were created: male-control $(n=18)$, male-PS $(n=11)$, female-control $(n=11)$, and female-PS $(n=13)$. Weight of the offspring was monitored regularly throughout the experiment, serving as an indication of general wellbeing.

\subsection{Behavioral testing}

Due to aggressive behavior among the males all animals were housed individually from 3 months of age onwards. Animals were put on a reversed day/night cycle (lights on from 7.00h P.M to 7:00h A.M.) and allowed to habituate for two weeks prior to the start of the 
behavioral testing, which was always performed in the dark phase. Animals were on average 7 months of age during cognitive testing. To assess cognitive alterations, the OLT, the Ymaze spontaneous alternation test and the $Y$-maze spatial memory test were used, while affective behavior was assessed with the EZM and the FST (see Figure 1 for a global timeline).

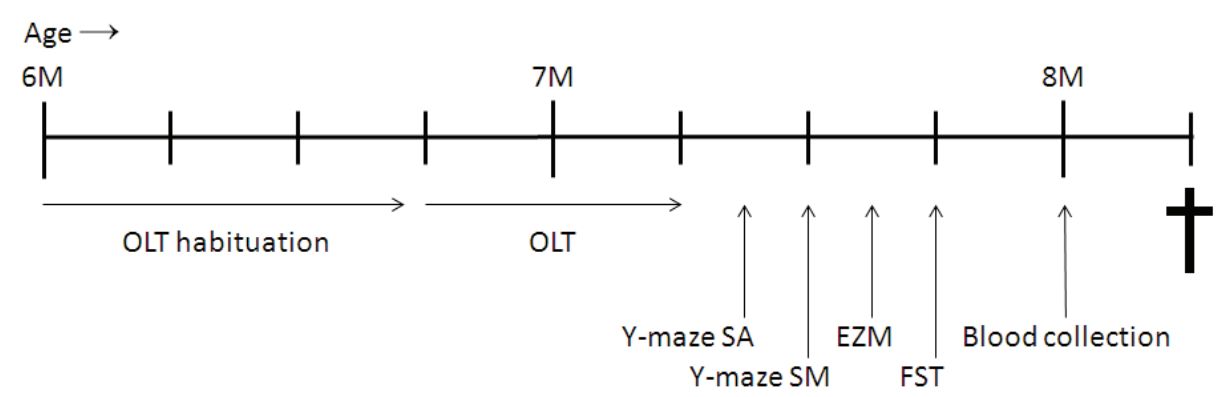

Figure 1: Timeline of behavioral testing. The age is annotated in months (M). OLT: object location task; Y-maze SA: Y-maze spontaneous alternation test; Y-maze SM: Y-maze spatial memory test; EZM: elevated zero maze; FST: forced swim test.

\subsubsection{Object location task}

Long-tem spatial memory was tested using the OLT, as described previously [522]. In brief, the setup consisted of a circular arena, $43 \mathrm{~cm}$ in diameter. The $40 \mathrm{~cm}$ high wall was divided into two halves, one made of white polyvinyl chloride, the other made of transparent polyvinyl chloride. Two objects were placed symmetrically in the middle of the arena. Four different sets of objects were used, and each object was available in triplicate. The different objects were: 1) a massive metal cube $(2.5 \times 5 \times 7.5 \mathrm{~cm})$ with two holes (diameter $1.5 \mathrm{~cm}$ ), 2) a white plastic cube with rounded off edges placed on a small stand of the same material (maximal diameter $3.5 \mathrm{~cm}$ and total height $11.5 \mathrm{~cm}$ ), 3) a brass cylinder with a tapering top (maximal diameter $3 \mathrm{~cm}$ and total height $11.5 \mathrm{~cm}$ ) and 4) a massive aluminum cube with a tapering top $(4.5 \times 4.5 \times 8.5 \mathrm{~cm})$. The objects could not be displaced by the mouse. The task was conducted as previously described $[522,523]$. To habituate the animals to behavioral testing, all mice were first handled, weighed and were able to explore the empty arena of the OLT for $4 \mathrm{~min}$. Subsequently, the animals were habituated to the 1 hour $(\mathrm{h})$ and $4 \mathrm{~h}$ interval of the OLT. A testing session consisted of two trials, with each trial lasting 4 min. During the first trial (T1) the arena contained two identical objects at fixed locations. A mouse was introduced into the arena by placing it with its nose to the middle of the transparent segment of the wall. After the first exploration period had elapsed, the mouse was placed back in its home cage. Subsequently, after a predetermined time interval (e.g. $1 \mathrm{~h}$ or $4 \mathrm{~h}$ ) the mouse was put back into the arena for the second trial (T2). However, now one of the objects had 
shifted location, which was randomized in a balanced manner over the groups and the different sessions to reduce potential bias due to preference for particular objects [523]. The time spent exploring the objects during T1 and T2 was recorded manually using a computer. Exploring behavior was defined as follows: directing its nose to the object at a distance of no more than $1 \mathrm{~cm}$ and/or touching the object with its nose. Sitting on the object was not considered exploration. In order to avoid the presence of olfactory cues, all objects and the arena were cleaned thoroughly with ethanol between sessions. In order to determine whether a mouse had remembered the familiar location of the object, several measures were used. The amount of exploration time is recorded in T1 (named e1) and T2 (e2). The discrimination index $\mathrm{d} 2$ calculates whether the mouse spends more time at the novel location while correcting for the total e2: (exploration time new location - exploration time old location)/e2. The latter will be an indication of recognition of the novel location of the objects, i.e. spatial memory. A minimum of 1.5 seconds exploration was set as an inclusion criterion.

\subsubsection{Y-maze spontaneous alternation test}

Spatial working memory was assessed by using the $\mathrm{Y}$-maze spontaneous alternation test, as previously described [524]. The symmetrical grey $\mathrm{Y}$-maze made of acrylic consists of three arms, with all arms being apart from the others at a $120^{\circ}$ angle. Each arm was $40 \mathrm{~cm}$ long, $17 \mathrm{~cm}$ high, $4 \mathrm{~cm}$ wide at the bottom and $13 \mathrm{~cm}$ wide at the top. Each mouse was placed in the centre of the Y-maze and was free to explore the arena for 6 min. The number of entries was counted per mouse: one entry required that both hind paws of the animal had to be placed completely inside the arm. A mouse would be making a triad when it visited all three arms consecutively. As a measure for working memory, the percentage of alternations that the mouse made was calculated, being the number of triads divided by the maximum possible alternations (i.e. the total number of entries minus 2) $\times 100$. If a mouse scored significantly above $50 \%$ alternations (the chance level for choosing the unfamiliar arm), this was indicative of functional working memory. Between sessions, the maze was cleaned thoroughly with ethanol.

\subsubsection{Y-maze spatial memory test}

The Y-maze spatial memory test was performed using the same arena as described above and served as an additional measure for long-term spatial memory. In this version, 1 arm was made inaccessible due to a removable blockade placed in front of one arm [525, 526]. The maze was always cleaned in between sessions with ethanol to reduce olfactory biases. A mouse was placed in 1 of the open arms (termed the 'start arm', which was randomized over the groups) and allowed to explore the 2 open arms of the maze for $5 \mathrm{~min}$. It was then taken out of the arena and placed back into the home cage. After a $1 \mathrm{~h}$ interval, the mouse was placed back into its corresponding start arm of the arena, but now the blockade had been removed, providing access to the previously blocked arm (termed the 'novel arm'). Spatial memory was assessed by the amount of time spent in the novel arm, which had to 
be significantly more than $33.3 \%$, corrected for the latency to move from the start arm to another arm and the amount of time the animal spent in the center of the maze.

\subsubsection{Elevated zero maze}

The EZM is an effective tool to measure anxiety in a rodent [527]. The apparatus consisted of a circular runway (diameter $46 \mathrm{~cm}$, width $5.5 \mathrm{~cm}$ ) made of black plastic material which was transparent for infrared light and elevated $20 \mathrm{~cm}$ above the floor. The arena was divided into 4 quadrants: two opposing open quadrants with a border (height $3 \mathrm{~mm}$ ) to prevent the mouse from stepping down, and 2 opposing closed quadrants with side walls (height $11 \mathrm{~cm}$ ). Each mouse was placed individually into one of the open arms close to and facing a closed arm, and was allowed to explore the arena for 5 min under dark conditions. After each session the maze was thoroughly cleaned using ethanol and water. The movements of each mouse were tracked automatically using an infrared camera and Ethovision Pro version 3.0. (Noldus, Wageningen, The Netherlands) [528]. Parameters for anxious behavior were the total distance traveled and time spent in the closed arms, corrected for possible freezing behavior at the start of the experiment.

\subsubsection{Forced swim test}

The FST is a widely used behavioral task to study depressive-like behavior in rodents [529]. Under low light conditions, each mouse was individually placed into a Perspex cylinder (diameter $17 \mathrm{~cm}$, height $40 \mathrm{~cm}$ ) filled with $30^{\circ} \mathrm{C}\left(+/-2^{\circ} \mathrm{C}\right.$ ) water to a height of $15 \mathrm{~cm}$ for 6 min. The total distance swum was used as a measure of depressive-like behavior, i.e. a lesser distance travelled being indicative of heightened despair (see Supplementary Data Figure S1D). Each session was scored automatically with Ethovision Pro (Noldus, The Netherlands) [528].

\subsection{Blood sampling and corticosterone radioimmunoassay}

In order to determine HPA axis responsiveness in the face of a stressor, blood samples were taken to determine corticosterone concentrations in these mice. First, the mice were taken out of their home cage, immediately after which a blood sample was drawn from the vena saphena (basal corticosterone concentration) using heparinized blood collection tubes (Microvette ${ }^{\circledR}$ CB300, Sarstedt, Germany). Subsequently, the mouse was restrained in a glass cylinder to induce a stress response. After $20 \mathrm{~min}$ of restraint stress, another blood sample was drawn from the vena saphena (stress-induced corticosterone concentration). Animals were then placed back in their home cage and housing room and allowed to recover for 60 min after which a third sample was taken ('recovery' corticosterone concentration). All blood samples were kept on ice and subsequently centrifuged at $3000 \mathrm{rpm}$ for $10 \mathrm{~min}$ at $4^{\circ}$ $\mathrm{C}$, after which plasma was isolated, frozen down to $-80^{\circ} \mathrm{C}$ and stored until further processing. For the corticosterone radioimmunoassay, an ImmuChem ${ }^{\mathrm{TM}}$ Double Antibody Corticosterone ${ }^{125}$ I RIA Kit for rodents (MP Biomedicals, Orangeburg, NY, USA) was used. 
The assay was performed according to the manufacturer's instructions. Briefly, for each sample, $5 \mu \mathrm{l}$ serum was diluted in steroid diluents (1:100 for basal and recovery samples and 1:200 for stress samples). Per $50 \mu \mathrm{l}$ of diluted sample, $50 \mu \mathrm{l}$ of corticosterone ${ }^{-125} \mathrm{I}$ and $100 \mu \mathrm{l}$ anti-corticosterone was added, and samples were incubated at room temperature (RT) for $2 \mathrm{~h}$. Afterwards, $250 \mu \mathrm{l}$ precipitant solution was added and tubes were centrifuged at $2300 \mathrm{rpm}$ for $15 \mathrm{~min}$. Supernatant was aspirated and the precipitate was counted in a Wizard Gamma Counter 2470 (Perkin Elmer, Waltham, MA, USA).

\subsection{Tissue preparation}

Mice were sacrificed by intracardial perfusion using Tyrode's solution for the first minute, followed by Somogyi's fixation solution (4\% paraformaldehyde, $15 \%$ picric acid, $0.05 \%$ glutaraldehyde in $0.1 \mathrm{M}$ phosphate buffer) for $10 \mathrm{~min}$ under deep pentobarbital anesthesia (100 mg/kg). The brains were removed and post fixated for $2 \mathrm{~h}$ in Somogyi's fixation solution, but omitting the glutaraldehyde. Cryoprotection took place by immersion into $15 \%$ sucrose solution in $0.05 \mathrm{M}$ Tris-buffered saline (TBS, $\mathrm{pH} 7.6$ ) at $4^{\circ} \mathrm{C}$ overnight. Subsequently, the brains were quickly frozen using $\mathrm{CO}_{2}$ and cut at the cryostat into $30 \mu \mathrm{m}$ thick coronal sections in 10 series using a CM3050 cryostat (Leica, Wetzlar, Germany). All series were stored at $-80^{\circ} \mathrm{C}$ until further processing.

\subsection{Immunohistochemistry}

For all immunohistochemistry, rinsing in between incubation steps was performed 3 times for $10 \mathrm{~min}$ in TBS-T (TBS with $0.2 \%$ Triton-X100), TBS, and TBS-T, respectively, unless specified otherwise.

\subsubsection{Extracellular $A \beta$ immunoreactivity}

To determine extracellular hippocampal plaque load, a triple-fluorescent immunohistochemical staining was performed, using the $6 \mathrm{E} 10$ antibody to stain all $A \beta$, Thioflavine $S$ to stain the highly fibrillar dense-core plaques and Hoechst labeling as a counterstain. Sections were rinsed before overnight incubation with the primary anti-A $\beta$ 1-17 antibody (6E10 clone, 1:1200, Sigma, Zwijndrecht, the Netherlands) in TBS-T at $4^{\circ} \mathrm{C}$. Sections were subsequently rinsed and incubated for $2 \mathrm{~h}$ with Alexa 594 (donkey antimouse, 1:200, Breda, The Netherlands) in TBS-T at RT. After another rinse, sections were incubated for 10 min with Thioflavine S $(0.0075 \%)$ in TBS-T at RT. Next, the sections were rinsed for $5 \mathrm{~min}$ in $70 \%$ ethanol and then $10 \mathrm{~min}$ in TBS, before incubating them for $30 \mathrm{~min}$ with Hoechst (1:500, Sigma, Zwijndrecht, the Netherlands) in TBS at RT, after which the sections were mounted and coverslipped in $80 \%$ glycerol in TBS.

\subsubsection{Intracellular $A \beta$ immunoreactivity}

To assess the amount of intracellular $A \beta$ within the hippocampal cell layers, McSA1 immunohistochemistry was performed, binding to amino acids 1-12 of the $A \beta$ sequence 
[530, 531]. Free-floating sections were rinsed overnight in TBS at $4^{\circ} \mathrm{C}$, after which antigen unmasking was performed with $10 \mathrm{mM}$ sodium citrate buffer $(\mathrm{pH} 6.0)$ in a $95^{\circ} \mathrm{C}$ water bath for $20 \mathrm{~min}$, followed by a $1 \%$ hydrogen peroxide incubation for $1 \mathrm{~h}$ to quench endogenous peroxidase activity and $5 \%$ normal donkey serum in TBS-T incubation to block aspecific IgG binding. After 3 nights of incubation with primary mouse monoclonal anti-A $\beta$ antibody (McSA1; 1:500, MediMabs, Montreal, Canada) on a constant shaker at RT, sections were rinsed and incubated with biotinylated donkey anti-mouse (1:200, Jackson, Westgrove, PA, USA) in $0.5 \%$ normal donkey serum in TBS-T for $2 \mathrm{~h}$ at RT. After another rinse, sections were incubated with avidin-biotine-peroxidase complex (1:800, Vector Laboratories, Burlingame, CA, USA) for $2 \mathrm{~h}$ at $\mathrm{RT}$, rinsed 3 times in $0.05 \mathrm{M}$ Tris- $\mathrm{HCl}(\mathrm{pH} \mathrm{7.6)}$ and were developed using 3,3'-diaminobenzidine tetrahydrochloride (DAB) solution (1:1 DAB: Tris$\mathrm{HCl}, 0.3 \% \mathrm{H}_{2} \mathrm{O}_{2}$ ) (Sigma, Uithoorn, The Netherlands). The reaction was stopped after $10 \mathrm{~min}$ by rinsing the section with TBS after which they were mounted onto gelatin-coated glasses, dehydrated and coverslipped using Pertex (HistolabProducts ab, Göteborg, Sweden).

\subsubsection{Synaptophysin immunoreactivity}

As $A D$ is also characterized by synaptic dysfunction, presynaptic bouton integrity was analyzed using synaptophysin immunoreactivity. The sections were rinsed 3 times in TBS and incubated with $0.3 \% \mathrm{H}_{2} \mathrm{O}_{2}$ for $30 \mathrm{~min}$ at $\mathrm{RT}$ after which the sections were rinsed, and subsequently incubated with $5 \%$ normal donkey serum in TBS-T for $30 \mathrm{~min}$ at RT. Afterwards, sections were incubated overnight with mouse anti-synaptophysin antibody (1:200, Millipore, Amsterdam, the Netherlands) in TBS-T with $0.5 \%$ normal donkey serum at RT. Subsequently, sections were rinsed and incubated for $2 \mathrm{~h}$ with biotinylated donkey antimouse antibody (1:100) in TBS-T with $0.5 \%$ normal donkey serum. After another rinse, sections were incubated for $90 \mathrm{~min}$ with the avidin-biotin-peroxidase complex at RT. After incubation the sections were washed 3 times for $10 \mathrm{~min}$ in Tris- $\mathrm{HCl}$ and incubated for $10 \mathrm{~min}$ with 1:1 DAB:Tris- $\mathrm{HCl}$ solution. The reaction was stopped after $10 \mathrm{~min}$ by rinsing the section with TBS after which they were mounted onto gelatin-coated glasses, dehydrated and coverslipped using Pertex.

\subsubsection{DNMT3a, $5 m \mathrm{~m}$ and $5 \mathrm{hmC}$ immunoreactivity}

To assess changes in DNA methylation patterns, immunohistochemical stainings were performed on de novo DNMT3a, $5 \mathrm{mC}$ and $5 \mathrm{hmC}$. Free-floating sections were rinsed overnight in TBS at $4^{\circ} \mathrm{C}$, after which antigen unmasking was performed as explained in 2.7.2., followed by a $1 \%$ hydrogen peroxide incubation for $1 \mathrm{~h}$ and $5 \%(5 \mathrm{mC})$ or $2 \%(5 \mathrm{hmC})$ normal donkey serum in TBS-T incubation to block aspecific IgG binding. After overnight incubation with the primary antibody (rabbit polyclonal anti-DNMT3a (1:200), Santa Cruz, CA, USA; mouse monoclonal anti-5mC (1:200; 3 night incubation), Genway Biotech Inc., San Diego, CA, USA; anti-5hmC (1:25000), Active Motif, Rixensart, Belgium) on a constant shaker at RT, sections were rinsed and incubated with the appropriate secondary antibody 
(biotinylated donkey anti-rabbit (1:200); biotinylated donkey anti-mouse (1:200), Jackson, Westgrove, PA, USA) in $0.1 \%$ bovine serum albumin in TBS-T (DNMT3a), $0.2 \%(5 \mathrm{hmC})$ or $0.5 \%(5 \mathrm{mC})$ normal donkey serum in TBS-T for $2 \mathrm{~h}$ at RT. After another rinse, sections were incubated with avidin-biotine-peroxidase complex (1:400, Vector Laboratories, Burlingame, $\mathrm{CA}$, USA) for $2 \mathrm{~h}$ at $\mathrm{RT}$, rinsed 3 times in $0.05 \mathrm{M}$ Tris- $\mathrm{HCl}$ and were developed using DAB. The reaction was stopped after $10 \mathrm{~min}$ by rinsing the section with TBS after which they were mounted onto gelatin-coated glasses, dehydrated and coverslipped using Pertex.

\subsection{Analysis of immunoreactivity}

\subsubsection{Stereological assessment of extracellular $A \beta$ immunoreactivity}

All $A \beta$ immunostained sections containing hippocampal tissue, ranging from Bregma -1.34 $\mathrm{mm}$ to $-3.88 \mathrm{~mm}$ [532], were analyzed unilaterally using Stereo Investigator software (version 8.26, MBF Bioscience, Williston, VT, USA). Per animal, an average of 9 sections was analyzed, depending on the individual rostro-caudal extension of the hippocampus. Delineations of the total hippocampus were made using a $4 x$ objective $(N A=0.16)$ and perfected using a $20 x$ objective (oil, $N A=0.80$ ). Within the delineation, the plaque area was measured with the Area Fraction Fractionator probe of the Stereo Investigator software using a 40x objective (oil, NA=1.00). Thioflavine-S immunoreactivity indicated the presence of dense-core plaques, while 6E10 immunoreactivity could demonstrate the less fibrillar plaque load often surrounding the dense core. All measurements were performed using a modified BX50 fluorescence microscope (Olympus, Tokyo, Japan), Olympus UPlanApo objectives (Olympus, Tokyo, Japan), three-axis high-accuracy computer-controlled stepping motor specimen stage for automatic sampling (4x4 Grid Encoded Stage; Ludl Electronics, Hawthorne, NY, USA), linear z-axis position encoder (Ludl), MBF-CX9000 CCD color camera (1.200x1.800 pixels; CX9000; MBF Bioscience) and controlling software (MBF Bioscience, Williston, VT, USA). The plaque area was expressed as a percentage of the total hippocampal area of that respective section. During statistical analysis, a distinction was made between the dorsal (Bregma $-1.34 \mathrm{~mm}$ to $-2.30 \mathrm{~mm}$ ) and ventral (Bregma -2.92 $\mathrm{mm}$ to $-3.88 \mathrm{~mm}$ ) hippocampus (adapted from [533]).

\subsubsection{Image analysis of synaptophysin immunoreactivity}

All synaptophysin immunostained sections containing hippocampal tissue, ranging from Bregma $-1.34 \mathrm{~mm}$ to $-3.64 \mathrm{~mm}$, were analyzed unilaterally. Per animal, an average of 8 sections was analyzed, depending on the individual rostro-caudal extension of the hippocampus. Three subregions within the hippocampus were assessed, i.e. the stratum moleculare of the dentate gyrus (DG), the stratum lucidem of CA3 and the stratum radiatum of CA1. Within these regions, 3 randomly selected sites were evaluated. The density of the synaptophysin-immunoreactive presynaptic boutons (SIPBs) was estimated as previously described [534]. In short, an image was taken of these chosen areas using a 100x lens (oil, 
NA=1.35, Olympus UPlanApo, Olympus, Tokyo, Japan) with a digital camera (f-view; Olympus, Tokyo, Japan) connected to an Olympus AX70 brightfield microscope (analySIS; Imaging System, Münster, Germany). In these images (on average 72 per animal), SIPBs were detected by CellP, an image analysis system, slightly modified for detection of grayscale punctae (AnalySIS-pro, Münster, Germany). In every image, the region of interest was defined and shading error correction was performed before measurements to correct for irregularities in illumination of the microscopic fields. Next, a differential contrast enhancement filter (DCE) was applied to selectively enhance weak differences in contrast and the detection threshold was tested and kept at the same level for all samples. All measurements were performed on a single focal plane and cell bodies, blood vessels and artifacts, e.g. A $\beta$ plaques were excluded from the analysis [267]. Particles smaller than 0.025 $\mu \mathrm{m}^{2}$ and larger than $10 \mu \mathrm{m}^{2}$ were considered noise and not taken into account in the analysis. From these data the mean SIPB density per $\mu \mathrm{m}^{2}$ was calculated.

\subsubsection{Image analysis of intracellular $A \beta, D N M T 3 a, 5 m C$ and $5 \mathrm{hmC}$ immunoreactivity}

The level of immunoreactivity per image was determined by measuring the mean grey value of immunoreactivity (reflecting the intensity) and the surface area of the delineated regions occupied by either McSA1, DNMT3a, $5 \mathrm{mC}$ or $5 \mathrm{hmCimmunoreactivity.} \mathrm{Five} \mathrm{hippocampal}$ sections were selected per animal, 2 in the dorsal (between Bregma -1.34 and -2.18), 1 in the middle (between Bregma -2.30 and -2.80 ), and 2 in the ventral hippocampus (between Bregma -2.92 and -3.88) [532], and 11 pictures were taken per section: 5 images of the granular cell layer of the DG, 3 images of the pyramidal cell layer of the CA3 subregion, and 3 images of the pyramidal cell layer of the CA1-2 subregion, encompassing a total of 55 images per animal. Images were taken using the microscope and camera as described under section 2.8.2., except a 40x objective was used (oil, NA=1.00, Olympus UPlanApo) The mean grey value and surface area were assessed in each picture, omitting hippocampal neuropil, staining artifacts, and type II DNMT3a immunoreactive cells (when applicable), by using the ImageJ software program (version 1.42q, Wayne Rasband, National Institutes of Health, Bethesda, Maryland, USA). Background corrections were performed by setting the same minimum threshold value for every image as described previously [535]. Mean grey value was defined as the sum of grey values of all pixels within the delineated area divided by the number of all pixels in this region. The surface area was defined as the percentage of the delineated area with a grey value above the background threshold.

\subsubsection{Density of type II intense DNMT3a-immunoreactive cells}

DNMT3a immunoreactivity is expressed in two different patterns, reflecting distinct cell types. The first type is present in the nucleus of cells throughout the neuronal cell layer and show moderate levels of DNMT3a immunoreactivity (type I DNMT3a immunoreactive cells), while the second type shows very intense DNMT3a immunoreactivity and is present only in a subset of hippocampal cells (type II DNMT3a immunoreactive cells) (see Figure 8A-B and 
[536] for further details on DNMT3a immunoreactivity). All measurements of type II intense DNMT3a immunoreactive cells were performed on the same stereology working station and software that was described in section 2.8.1., but now using bright field instead of fluorescence. First, the entire hippocampus was delineated using a 10x lens (NA=0.40), and estimates of the total number of cells were performed using the optical fractionator [537, 538] and a 40x lens (oil, NA=1.00). Cell densities were estimated for all hippocampal sections per animal (average of 8 sections) by calculating the ratio of the number of cells counted in each section, divided by the area of the hippocampus in the corresponding sections [539]. No hippocampal volume differences were found between the groups (data not shown).

\subsection{Statistical analysis}

All data are presented as mean and standard error of the mean (S.E.M.). Two-way ANOVA was performed for comparison between groups accounting for the main and interactive effects of sex (male, female) and treatment (control, PS). When an interaction or main effect was observed, the data was stratified for sex and a student's t-test was performed to assess whether treatment effects were specific for, or more pronounced in a certain sex, as this was expected, based on literature $[511,514]$. Corticosterone concentrations were LNtransformed before performing Repeated Measures ANOVA to assess the main and interactive effects of sex and treatment over the different conditions as well as per condition separately. OLT discrimination performance was assessed using a one-sample t-test with zero as the testing value. For the $\mathrm{Y}$-maze spontaneous alternation test, the one-sample ttest testing value was set at $50 \%$ and at $33.3 \%$ for the spatial memory variant of the maze. Statistical significance was set at an $\alpha$ level of 0.05 and all tests were two-tailed. All statistical analyses were conducted using the Statistical Package for the Social Sciences (SPSS 15.0, SPSS Inc., Chicago, IL, USA). All graphs were constructed in GraphPad Prism (version 4, GraphPad Software, San Diego, CA, USA).

\section{Results}

\subsection{Object location task}

The OLT was used to assess alterations in long-term spatial memory as a consequence of PS exposure. When investigating whether the $d 2$, as a measure for spatial memory function, of each individual group was significantly higher than 0 (i.e. no recall), thereby indicative of functionally intact spatial memory, it appeared that during the $1 \mathrm{~h}$ interval, only the malecontrol $(t(13)=3.36, p<0.01)$ and female-PS $(t(10)=3.27, p<0.01)$ groups were significantly able to distinguish the novel from the familiar location. Interestingly, the female-PS group was also able to significantly distinguish the novel from the familiar location at the $4 \mathrm{~h}$ interval $(t(9)=2.69, p<0.05)$ (see Supplementary data, Figure S1A). 
When comparing discrimination performance between groups, a significant sex*treatment effect could be observed with regards to the $\mathrm{d} 2$ discrimination index of the $1 \mathrm{~h}$ interval $(F(1,40)=5.60, p<0.05)$, with male PS mice performing worse and female PS mice appearing to perform better, when compared to the corresponding controls. After stratifying for sex, it was demonstrated that PS significantly decreased the $d 2$ in male mice $(F(1$, $21)=4.93, p<0.05$ ), while having no significant effect on the $d 2$ score in female mice (see Figure 2). No main effect for sex or treatment was observed for the $\mathrm{d} 2 \mathrm{~d}$ of the $1 \mathrm{~h}$ interval, and the $\mathrm{d} 2$ score of the $4 \mathrm{~h}$ interval also remained unaffected affected by sex, treatment or sex*treatment. An overview of the exploration times during the $1 \mathrm{~h}$ and $4 \mathrm{~h}$ interval are listed in the Supplementary Data (see Table S1).

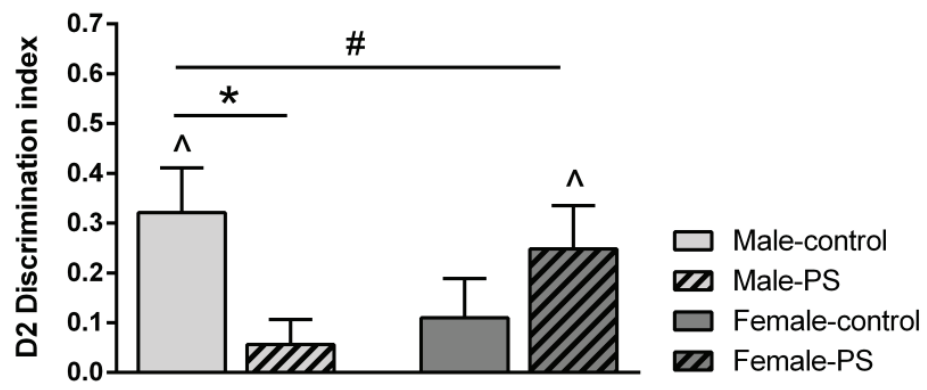

Figure 2: Scores on the d2 discrimination index during the $1 \mathrm{~h}$ interval of the object location task (OLT). Bars indicate mean and S.E.M. per group. A significant sex treatment effect could be observed, with PS male showing a worse performance after PS, while females appeared to benefit from PS. This interaction effect was largely explained by a significant difference in $\mathrm{d} 2$ score between the male mice, with PS males scoring significantly lower. When comparing their performance to 0 (having no memory of the familiar location), it appears that both the male control and the female PS groups were able to distinguish the familiar from the novel location. \#: significant interaction effect, $p<0.05$; *: The male-PS group scored significantly lower than the male-control group, $p<0.05 ; \wedge$ : performance is significantly better from 0 , indicative of functionally intact spatial memory, $p<0.05$.

\subsection{Y-maze}

\subsubsection{Y-maze spontaneous alternation test}

To assess spatial working memory function, all mice were subjected to the Y-maze spontaneous alternation test. All groups scored significantly higher than the $50 \%$ threshold $(t(>8)>2.59, p<0.05)$, thereby indicating that their spatial working memory was intact (see Supplementary Data, Figure S1B). No main or interaction effects could be found.

\subsubsection{Y-maze spatial memory test}

The Y-maze spatial memory test served as an additional measure for long-term spatial memory assessment. Only the male-control group spend more than 33.3 percent of its time 
in the novel arm after a $1 \mathrm{~h}$ interval $(\mathrm{t}(17)=3.16, \mathrm{p}<0.01)$, while the other groups did not (see Figure 3). No interaction or main effects could be observed.

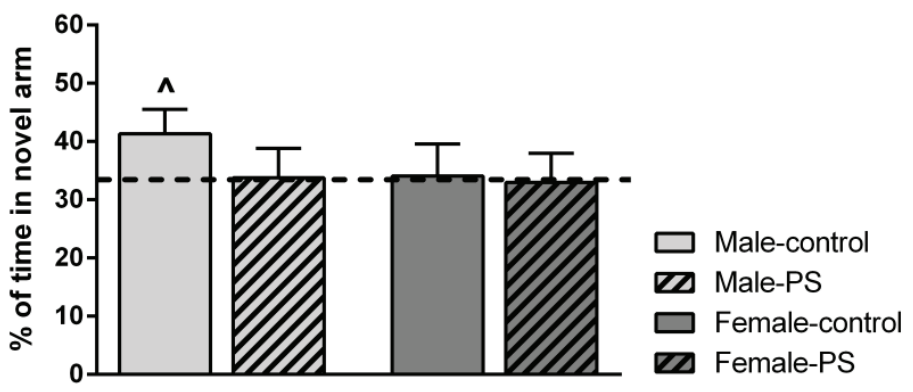

Figure 3: Percentage of time spent in the novel arm of the Y-maze spatial memory test. Bars indicate mean and S.E.M. per group. Only the male-control group spent significantly more time in the novel arm after a $1 \mathrm{~h}$ interval than could be explained by chance. ${ }^{\wedge}$ : $p<0.05$, compared to $33.3 \%$.

\subsection{Elevated Zero Maze}

The EZM was used to determine whether anxiety behavior was altered as a consequence of PS exposure. The percentage of time spent in the open arms of the EZM was not affected by sex, treatment nor sex*treatment, indicating that all animals showed similar levels of anxiety (see Supplementary Data, Figure S1C).

\subsection{Forced Swim Test}

To assess the effects of PS on induction of a more depressive-like phenotype, the FST was conducted. Analysis of the total distance swum in the FST demonstrated a significant sex*treatment $(F(1,49)=4.65, p<0.05)$ and a trend for a treatment effect $(F(1,49)=4.05$, $0.05<p<0.1)$. When stratifying for sex, it became clear that PS decreased the distance swum in females $(F(1,21)=7.71, p<0.05)$, but not in males (see Figure 4). Scoring immobility, i.e. making no movement or such minimal movement necessary to keep the nose above water, demonstrated a strong negative correlation with the distance swum in the FST for mice (see Supplementary Data, Figure S1D). 


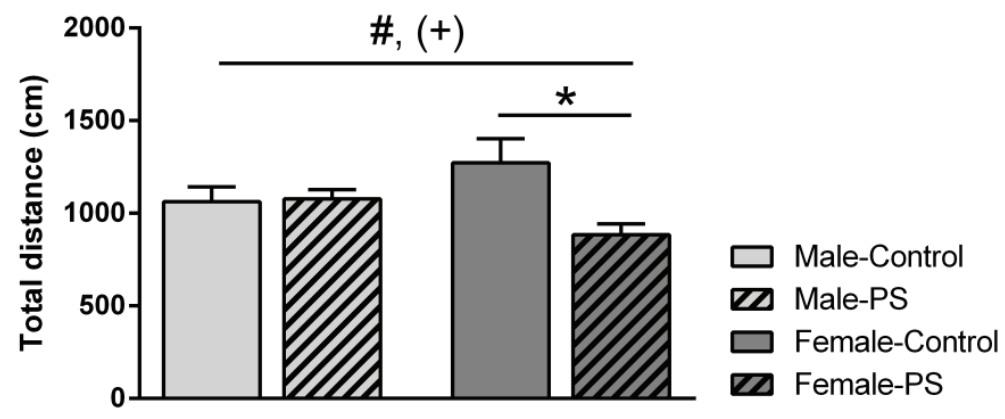

Figure 4: Total distance swum in the forced swim test. Bars indicate mean and S.E.M. per group. PS decreased total distance swum specifically in female mice. \#: significant sex*treatment effect, $p<0.05$; $(+)$ : trend for treatment effect, $0.05<p<0.1$; *: significant difference female-control and female-PS group, $\mathrm{p}<0.05$.

\subsection{Corticosterone concentrations}

Alterations in HPA axis reactivity were explored by drawing blood samples during basal, stressful and recovery conditions and determining plasma corticosterone concentrations. Plasma corticosterone concentrations were significantly influenced by condition (i.e. basal, stress, recovery) $(F(2,60)=88.09, p<0.01)$ and condition*sex*treatment $(F(2,60)=5.15$, $\mathrm{p}<0.01)$, but not by condition*sex or condition*treatment. An overall sex effect on corticosterone concentrations was observed $(F(1,30)=31.55$, $p<0.001)$, with females having higher concentrations. In addition, a trend for a sex*treatment effect $(F(1,30)=3.93$, $0.1<p<0.05$ ), but no single overall effects for treatment was found.

When looking at corticosterone concentrations stratified for sex, it was shown that both condition $(F(2,24)=64.33, p<0.001)$ and condition*treatment $(F(2,24)=8.56, p<0.01)$ had a significant effect on corticosterone concentrations in males. In females, however, only a condition effect $(F(2,36)=43.18, p<0.001)$, but not a condition*treatment effect could be observed on the corticosterone concentrations in blood plasma.

When examining the effect of treatment in male mice per condition, it was demonstrated that PS significantly lowered the corticosterone concentration during the stressful $(F(1,22)=6.37$, $p<0.05)$ and the recovery condition $(F(1,15)=31.49, p<0.001)$, but not during the basal conditions (see Figure 5). 


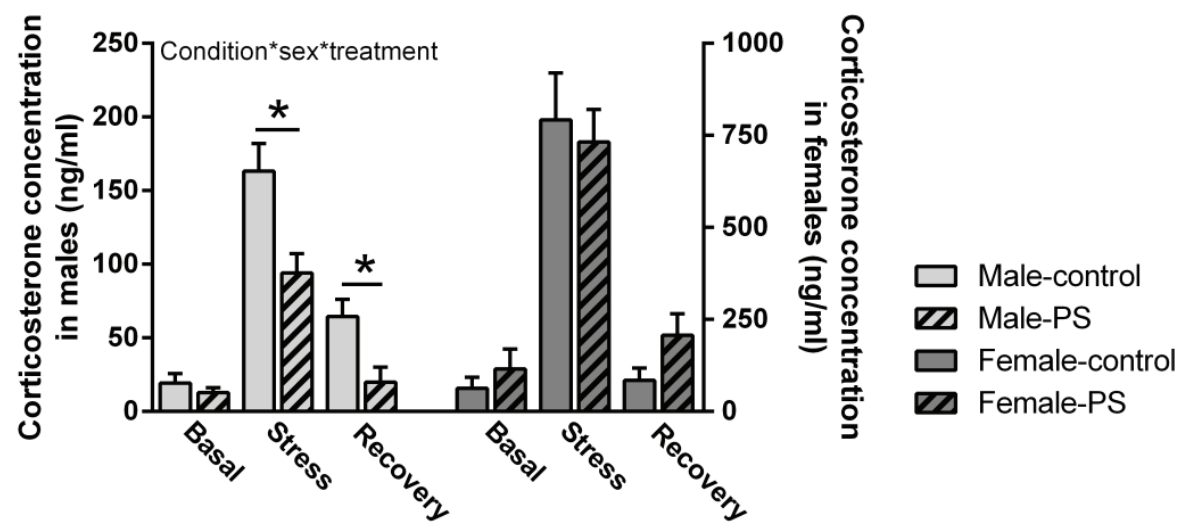

Figure 5: Plasma corticosterone concentrations during basal, stressful and recovery conditions. Bars indicate mean and S.E.M. per group, with corticosterone values of male mice depicted on the left vertical axis and values of females depicted on the right vertical axis. A significant condition*sex*treatment effect $(p<0.05)$ was found. In general, female mice had higher corticosterone concentrations over time than male mice, independent of condition. Male-PS mice showed a lower plasma corticosterone concentration during the stressful and recovery conditions than male-control mice. *: significant difference male-control and male-PS group, $p<0.05$.

\subsection{Extracellular $A \beta$ immunoreactivity analysis}

The extent of extracellular plaque deposition was assessed by quantifying extracellular $A \beta$ (6E10) immunoreactivity and Thioflavine S-positive plaques. Females demonstrated a $40 \%$ higher plaque load in the dorsal $(F(1,19)=6.21, p<0.05)$ and a $70 \%$ increase in the ventral $(F(1,18)=10.55, p<0.01)$ hippocampus, compared to male mice. Overall, PS exposure tended to lower plaque load in the dorsal $(F(1,19)=4.13,0.05<p<0.10)$, but not in the ventral hippocampus. When stratifying for sex, a $29 \%$ decrease in female dorsal plaque load was observed after PS exposure $(F(1,9)=6.43 ; p<0.05)$ (see Figure 6$)$. No sex treatment effects could be observed for the dorsal or ventral plaque load.

\subsection{Intracellular $A \beta$ immunoreactivity analysis}

Intracellular $A \beta$ immunoreactivity was assessed by specifically quantifying McSA1-ir (staining amino acids $1-12$ of the $A \beta$ sequence $[530,531]$ ) within the hippocampal cell layers. Analysis revealed that females had significantly lower McSA1 immunoreactivity in the dorsal CA3 $(F(1,22)=4.78, p<0.05)$, while a significant sex ${ }^{\star}$ treatment effect could be observed in the ventral CA3 $(F(1,21)=4.67, p<0.05)$. The other regions remained unaffected by sex, treatment of sex*treatment (see Figure 7E-F). Interestingly, a distinct difference in McSA1 immunoreactive surface area between the 3 subregions could be observed, where the percentage of the cell layer that was occupied with McSA1 immunoreactivity ranged from $12 \%$ in the DG, to $22 \%$ and $40 \%$ in the CA3 and CA1, respectively (see Figure $7 B-D$ ). No 
significant differences were observed in the McSA1 surface area in any of the subregions (data not shown).

A

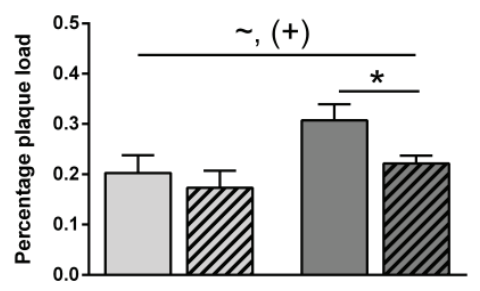

B

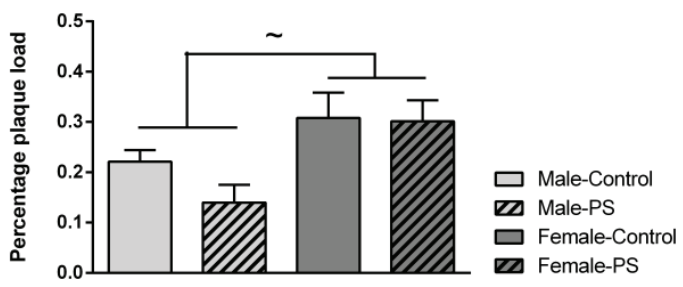

Figure 6: Analysis of plaque load in the dorsal (A) and ventral (B) hippocampus. Bars indicate mean and S.E.M. per group. Females generally had higher plaque load then male mice and PS significantly decreased plaque load in the dorsal hippocampus of female mice (A). : sign sex effect, $p<0.05 ;(+)$ : trend for treatment effect, $0.05<p<0.1$; *: significant difference female-control and female-PS, $p<0.05$.

\subsection{Synaptophysin immunoreactivity analysis}

Presynaptic bouton integrity was assessed by SIPB density measures per hippocampal subregion. SIPB density in the dorsal and ventral stratum moleculare of the DG, the stratum lucidem of $\mathrm{CA} 3$ and the stratum radiatum of CA1 were not different between the various groups (see Supplementary Data, Figure S2).

\subsection{DNMT3a immunoreactivity analysis}

\subsubsection{DNMT3a immunoreactivity analysis of type I cells}

DNMT3a is a marker for establishment of de novo DNA methylation. Females had significantly lower immunoreactivity of DNMT3a in type I cells in the dorsal DG $(F(1$, $22)=7.13, p<0.05$ ), while no effects of treatment or sex ${ }^{*}$ treatment could be observed. In the ventral $D G$, a tendency towards a sex and sex*treatment effect was seen $((F(1,21)=3.77$, $0.05<p<0.1),(F(1,21=4.27,0.05<p<0.1)$, respectively $)$, while no effect for treatment was found. The dorsal and ventral CA3 and CA1 subregion remained unaffected as a result of sex, treatment and sex*treatment (see Figure $8 \mathrm{G}-\mathrm{H}$ ). It was noted that in some animals the tip of DG showed an apparent lower amount of immunoreactivity than the upper and lower blade, and this area was therefore analyzed separately. It appeared that sex had a significant effect on DNMT3a immunoreactivity intensity $(F(1,22)=9.18, p<0.01)$, with females having lower DNMT3a immunoreactivity (see Supplementary Data, figure S3A-B). Treatment and sex*treatment had no effect on DNMT3a immunoreactivity in the tip of the DG. Analysis of the surface area occupied by DNMT3a immunoreactivity did not reveal any significant differences in neither of the subregions (data not shown). 


\subsubsection{Type II intense DNMT3a immunoreactive cells analysis}

Previous data has indicated that proliferating cells also display type II intense DNMT3a immunoreactivity (see [536]). With respect to the cell density of these type II intense DNMT3a immunoreactive cells within the hippocampus, it was shown that there were no statistical difference between the groups (see Supplementary Data, figure S3C).

\subsection{5m immunoreactivity}

Global DNA methylation status was assessed with $5 \mathrm{mC}$ immunoreactivity within the different hippocampal subregions. The interaction of sex treatment tended to affect $5 \mathrm{mC}$ immunoreactivity intensity in the dorsal DG $(F(1,21)=3.08,0.05<p<0.1)$, while a strong tendency for main treatment effect was observed in the ventral $D G(F(1,20)=4.385$, $0.05<p<0.1)$. No other main effects or interaction effects were found for the intensity in these regions, nor in the dorsal and ventral CA3 and CA1 subregions (see Figure 8I-J). Surface area analysis of $5 \mathrm{mC}$ could not provide any significant differences between the groups in neither of the subregions (data not shown).

\subsection{5hm C immunoreactivity}

Hydroxymethylation was assessed by $5 \mathrm{hmC}$ immunoreactivity, a DNA demethylation intermediate. Analysis of the mean grey value of $5 \mathrm{hmC}$ immunoreactivity in the dorsal and ventral DG, CA3 and CA1 subregions of the hippocampus, demonstrated that sex, treatment and sex ${ }^{\star}$ treatment did not have any effect (see Figure $8 \mathrm{~K}-\mathrm{L}$ ). The surface area occupied by $5 \mathrm{hmC}$ immunoreactivity was not different between the groups in neither of the subregions (data not shown). 

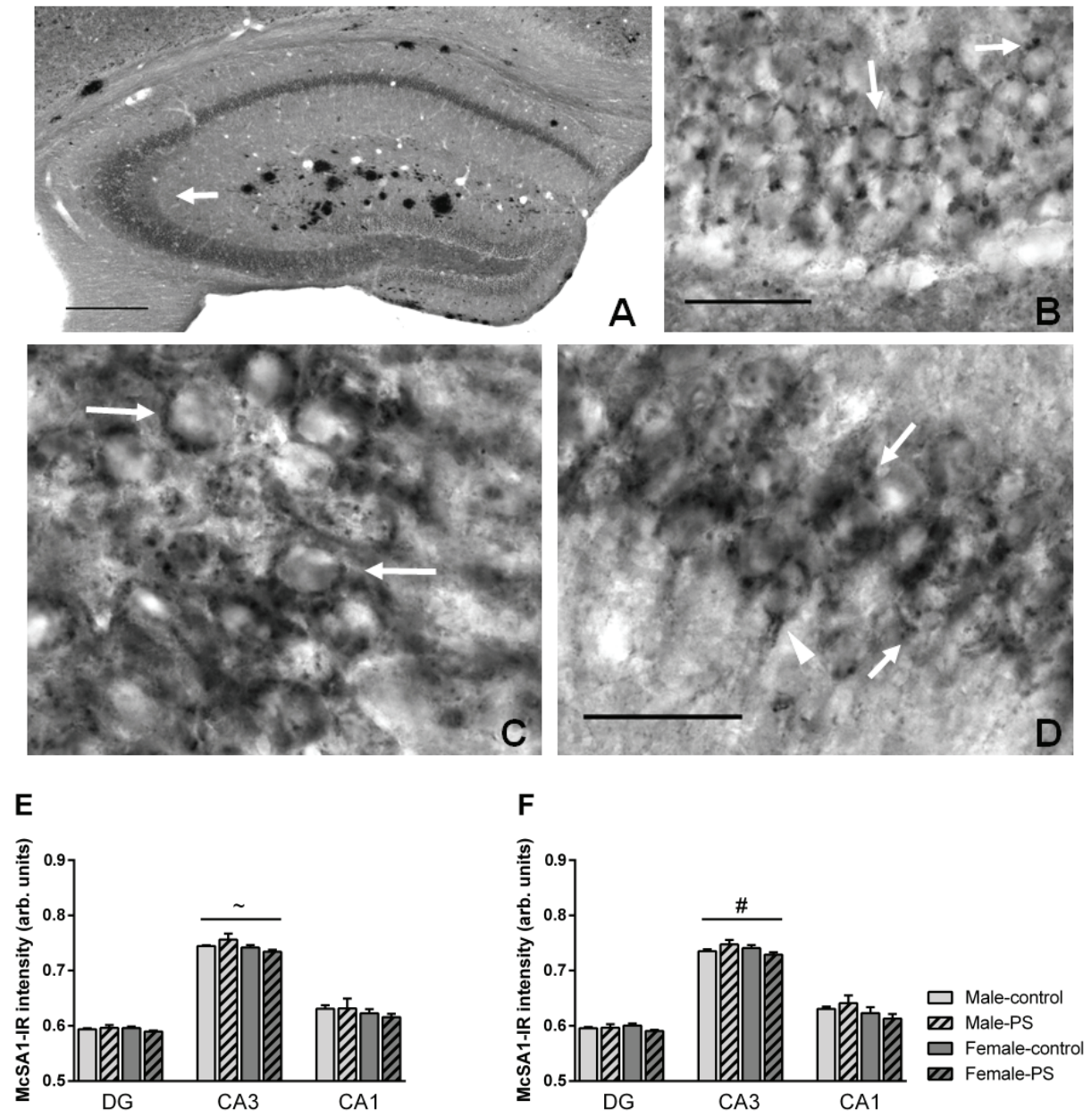

Figure 7: Intensity of McSA1-immunoreactivity in the hippocampus, staining $A \beta 1-12$ [531]. Representative images of McSA1 immunoreactivity in the total hippocampus (A) at 10x, the DG (B), the CA3 (C) and the CA1 (D), all in 100x. Scale bars: $500 \mu \mathrm{m}(\mathrm{A}), 50 \mu \mathrm{m}(\mathrm{B}, \mathrm{D})$. Note that McSA1 immunoreactivity is observed throughout the hippocampus both in amyloid plaques as well as within the hippocampal cell layers. Interestingly, clear McSA1 staining was also observed in the stratum lucidem of the CA3 layer (white arrow). The degree of McSA1 immunoreactivity is highly region-specific, with very little McSA1 immunoreactivity in the DG, and more profound McSA1 immunoreactivity in the CA3 and CA1 subregions. The distribution within the neuron is highly granular (white arrows in B-D), and was also abundantly present in the basal neurites (white arrowhead in D). Intensity of McSA1 immunoreactivity was quantified for the DG, CA3 and CA1 separately, in both the dorsal (E) and ventral (F) hippocampus. Bars indicate mean and S.E.M. per group. McSA1 immunoreactivity was significantly lower in female APPswe/PS1 $\triangle \mathrm{E} 9$ mice in the dorsal CA3 (E), while a sex*treatment effect was observed in the ventral CA3 (F); : sign sex effect, $p<0.05$; \#: significant sex*treatment effect, $p<0.05$. 

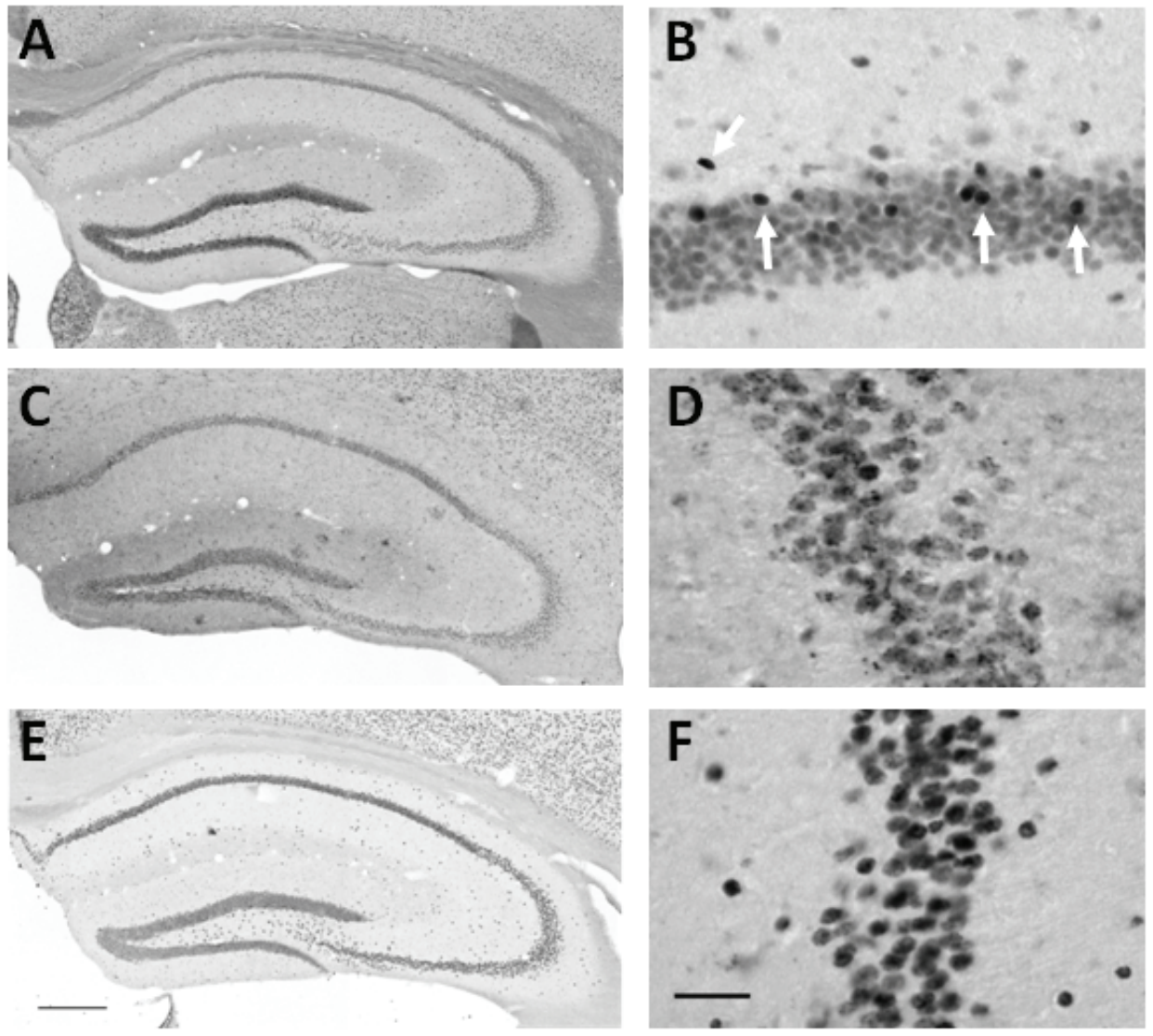

Figure 8: DNA methyltransferase 3a (DNMT3a), 5-methylcytidine $(5 \mathrm{mC})$, and 5-hydroxymethylcytidine $(5 \mathrm{hmC})$-immunoreactivity in the hippocampus. Representative images of DNMT3a immunoreactivity (A, B), $5 \mathrm{mC}(\mathrm{C}, \mathrm{D})$ and $5 \mathrm{hmC}(\mathrm{E}, \mathrm{F})$ in the total hippocampus at 10x and in the DG for DNMT3a (B), and the CA3 for 5-mC (D) and 5-hmC (F) in 40x. Scale bars: $500 \mu \mathrm{m}(E), 50 \mu \mathrm{m}(\mathrm{F})$. The white arrows in $B$ indicate cells with very intense DNMT3a immunoreactivity, i.e. type II DNMT3a-immunoreactive cells, which do not only comprise neurons. Intensity of DNMT3a, $5 \mathrm{mC}$, and $5 \mathrm{hmC}$ immunoreactivity was quantified for the DG, CA3 and CA1 subregions separately, distinguishing between the dorsal (G, I, K) and ventral $(\mathrm{H}, \mathrm{J}, \mathrm{L})$ hippocampus. Bars indicate mean and S.E.M. per group. Females showed significantly less DNMT3a immunoreactivity in the dorsal DG $(G)$, while in the ventral DG trends for a sex and sex*treatment effect were found, with males having higher and female lower DNMT3a immunoreactivity intensity values after PS $(\mathrm{H})$. In the $5 \mathrm{mC}$ staining only statistical trends were observed in the dorsal and ventral hippocampus, while no effects were found for $5 \mathrm{hmC}$ immunoreactivity intensity.. $\sim$ : sign sex effect; $(\sim)$ : trend for sex effect, $0.05<p<0.1 ;(+)$ : trend for a treatment effect, $0.05<p<0.1 ;(\#)$ : trend for sex ${ }^{*}$ treatment effect, $0.05<p<0.1$. 
G

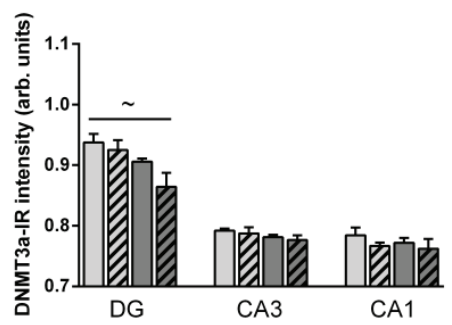

I

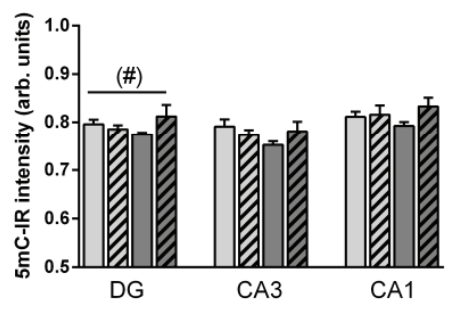

K

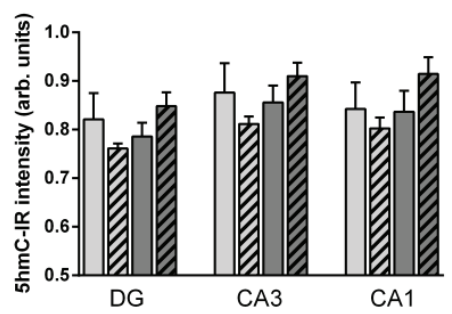

H

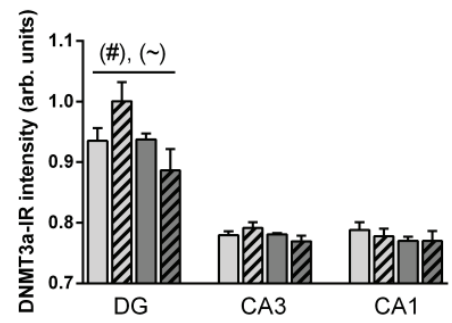

$J$

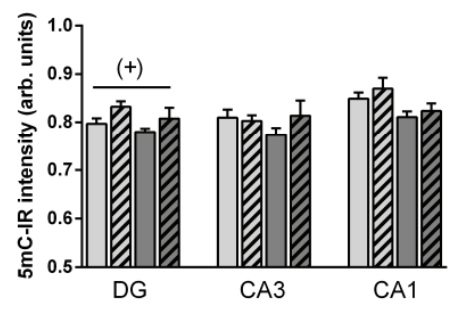

L

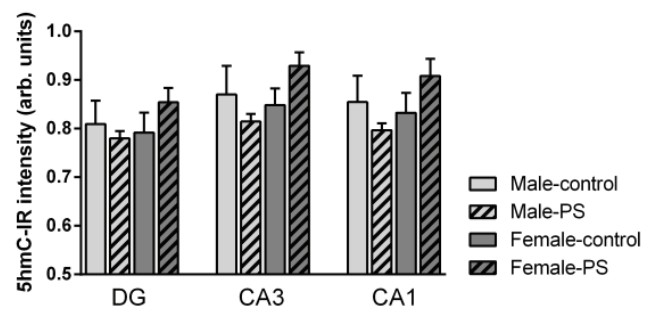

Figure 8: Continued

\section{Discussion}

In this study, we investigated the effects of PS on the onset and progression of AD-related behavioral deficits and associated neuropathology as well as epigenetic regulation, with an additional focus on potential sex-dependent differences. In short, it was shown that PS exerted sex-specific effects on cognitive performance, depressive-like behavior, HPA axis responsiveness and hippocampal plaque load. No effects of PS were seen on McSA1 or $5 \mathrm{mC}, 5 \mathrm{hmC}$ and DNMT3a immunoreactivity, although interesting sex differences were observed in some of these markers.

\subsection{PS exposure leads to sex-dependent effects on spatial memory}

Sex differences in spatial memory performance were clearly observed in the OLT, with female control mice not being able to distinguish between object locations after a 1 hour 
interval, while male control mice were. Interestingly, we found that PS exposure only impaired long-term spatial memory performance in male mice, whereas in contrast, PS exposure in females tended to exert a protective effect. These results are in line with Mueller \& Bale (2007) who found that early gestational stress in wild-type C57BL/6 mice was beneficial to female mice, while being detrimental to cognition in male mice [511]. Results from the $\mathrm{Y}$-maze spatial memory task confirmed that male spatial memory was impaired by PS exposure, although the improvement in female spatial memory performance by PS as observed in the OLT was not observed in the spatial Y-maze. This indicates that these 2 spatial tasks might rely on the function of slightly different brain circuits. In fact, the OLT requires the mouse to remember the spatial location of an external object, while in the spatial variant of the $\mathrm{Y}$-maze the subject needs to spatially navigate himself through the maze, which do indeed require the action of different processing pathways [540]. Finally, working memory did not seem to be impaired by PS exposure and appeared to be functioning properly in all groups, as has been reported before at this age of 7 months [52, 541].

\subsection{Depressive-like behavior and corticosterone secretion exhibit sex-dependent alterations in response to $P S$ exposure}

PS exposure also exerted a sex-dependent effect on affective behavior, as we demonstrated that only female mice displayed more depressive-like behavior after PS in the FST. Likewise, the same stress paradigm applied during E13-17 also elicited more depressive-like behavior in female mice only [510, 542]. In contrast, anxious behavior remained unchanged in both sexes.

The neuroendocrine secretion of corticosterone was also shown to be sex-specific. In general, female mice had much higher plasma corticosterone concentrations during basal, stressful and recovery conditions than males, independent of stress exposure, which is in accordance with literature on $A D$ patients [543] and other (AD) mouse models [150, 510, $542,544,545]$. PS did significantly affect corticosterone levels of male APPswe/PS1 $1 \mathrm{E} 9$ mice as these mice showed a blunted stress-induced HPA axis response and a faster recovery from the stressful event than their control counterparts.

Blunted HPA axis responsiveness after PS exposure has been previously reported in rodent offspring with concomitant affective and cognitive deficits [546-549], indicating that also HPA axis hypoactivity can lead to changes in the behavioral phenotype. The fact that PS exerts a sex-specific effect on HPA axis alterations has been widely established [548553]. Additionally, it has been shown that the degree and direction (hypo- or hyperactivity) of HPA axis alterations are highly dependent on the age at which the offspring is investigated $[549,554]$. We were not able to find any statistical correlations between corticosterone concentrations and behavior. As our results indicate that both sexes exhibit an altered behavioral phenotype in response to PS exposure, but not both sexes display alterations in HPA axis functioning at this given time point, it can be concluded that the behavioral 
alterations after PS do not solely depend on changes in HPA axis reactivity. It is therefore likely that the concept of prenatal programming within the PS field extends beyond HPA axis regulation and could be mediated via alternative mechanisms such as epigenetic regulation of genes involved in affective behavior [555].

\subsection{PS exposure leads to decreased hippocampal plaque load and altered APP processing in the female APPswe/PS1 $1 E 9$ mouse, without affecting SIPB density}

Sex differences in the APPswe/PS1 $\triangle \mathrm{E} 9$ mouse did not constrict themselves to behavioral and neuroendocrine phenotypes. With respect to AD-related neuropathology and morphology, our results showed that female APPswe/PS1 $\triangle \mathrm{E} 9$ mice had on average $65 \%$ more hippocampal plaque load than male mice of the same genotype and age, which corresponds with earlier observations [556]. In addition, PS exposure significantly decreased plaque load in the dorsal hippocampus, but only in female mice. Markedly, female mice with the lowest plaque load also performed best in the $1 \mathrm{~h}$ interval of the OLT, while in males no such association could be observed. This suggests that in females reduced $A \beta$ plaque deposition in response to PS exposure could contribute to the observed cognitive improvement, while in PS males the spatial memory impairments appear to be induced by other factors than amyloid burden. It has indeed been observed before in rat models of PS that male rats showed higher vulnerability for degeneration of hippocampal dendritic and spine morphology than female rats, possibly accounting for the observed learning and memory deficits in the male sex after PS exposure (reviewed in [557]). Given the broad range of sex-specific alterations in brain morphology and function as a result of PS exposure $[510,553,557-560]$, it is not surprising that plaque load is also affected in a sex-specific manner after PS.

However, the observation that gestational stress exposure significantly decreased dorsal hippocampal plaque load in female mice was unexpected, as other types of stress exposure in $A D$ mouse models demonstrated an increase in $A \beta$ neuropathology. These models used restraint or isolation stress during puberty and/or adulthood, and found elevated concentrations of $A \beta 40$ and $A \beta 42$ in the hippocampus and neocortex, increased hippocampal plaque load and increased neurodegeneration, combined with more cognitive deficits [76-78, 131, 134-136, 505, 506, 508]. It was also established that these stressinduced alterations in AD-related neuropathology could be mediated via changes in corticotropin-releasing factor [76], a key player in HPA axis regulation. Yet, our study demonstrates that PS-induced changes in plaque load occur independently of alterations in HPA axis functioning, as PS-treated female mice do not display HPA axis alterations. It can be concluded that stress exposure during adolescence or adulthood appears to exert quite a different effect on $A \beta$ production and deposition when compared to prenatal exposure. Following the LEARn model proposed by Lahiri et al. [80], it could be speculated that PS exposure, by means of fetal programming, exerts long-term effects on the expression and 
function of genes and associated proteins involved in $A \beta$ production and/or clearance, a process that could be mediated via changes in epigenetic regulation.

To investigate whether PS could lead to changes in APP processing, thereby underlying the alterations in extracellular amyloid deposits, intracellular $A \beta$ immunoreactivity was quantified (using the McSA1 antibody). To our knowledge, this is the first report that quantifies exclusively the intracellular immunoreactivity of an $A \beta$ marker. Although it is well established that the McSA1 antibody (staining $A \beta 1-12$ ) does not cross-react with the fulllength APP holoprotein or with its N-terminal products, such as soluble APPa [561, 562], the absence of cross-reactivity with the $\beta$-C-terminal fragment C99 needs to be further investigated. Whether or not McSA1 immunoreactivity purely represents changes in $A \beta$ or a combination of $A \beta$ and $C 99$ fragments, alterations in APP processing can still be visualized with this antibody. Interestingly, distinct differences in intracellular McSA1 immunoreactivity were observed within the hippocampal subregions of APPswe/PS1 $\triangle E 9$ mice, with very little immunoreactivity in the $D G$ and much more pronounced staining in the CA areas, perhaps reflecting region-specific $A \beta$ production. Nevertheless, female APPswe/PS1 $1 E 9$ mice had significantly lower McSA1 immunoreactivity within the dorsal CA3 cell layer than male mice, suggesting that APP processing in this region occurs in a sex-dependent manner. As PSinduced changes were observed in female extracellular plaque load, but not on the intensity of McSA1 staining, it would be interesting for future research to investigate the effects of PS exposure on possible alterations in $\mathrm{Y}$-secretase activity, or how the expression of this enzyme is epigenetically regulated. Altered $y$-secretase activity could be responsible for the observed discrepancy between McSA1 staining and extracellular plaques.

\subsection{Sexual dimorphism in DNMT3a immunoreactivity, but not in $5 \mathrm{mC}$ or $5 \mathrm{hmC}$}

Methylation patterns are known to be altered after PS exposure. It has, for example, been demonstrated that PS exposure may lead to increased DNA methylation of the promoter region of corticotropin releasing factor and decreased methylation of exon $1_{7}$ of the glucocorticoid receptor promoter, but only in male mice [514]. In addition, other prenatal perturbations such as intrauterine growth retardation due to bilateral uterine artery ligation, also affected global DNA methylation and histone acetylation patterns in a sexual dimorphic manner in the offspring [563]. To investigate whether PS exposure in the APPswe/PS1 $\triangle E 9$ mouse model would also affect global DNA methylation levels, we investigated immunoreactivity for DNMT3a, $5 \mathrm{mC}$ and $5 \mathrm{hmC}$ within the DG, CA3 and CA1 subregion of the hippocampus. Whereas DNMT3a mediates de novo methylation, and is mainly active during adult neurogenesis [564], global DNA methylation and hydroxymethylation levels were also assessed using antibodies against $5 \mathrm{mC}$ as well as the newly described $5 \mathrm{hmC}$ [565].

Analysis of DNMT3a immunoreactivity within the 3 different subregions of the hippocampus showed that DNMT3a immunoreactivity was highest in the CA3 and lowest in the DG subregion. Moreover, female mice demonstrated an even more pronounced reduction of DNMT3a immunoreactivity in the dorsal DG. This is remarkable as DNMT3a 
immunoreactivity and mRNA levels were found to be highest in the DG subregion in C57BL/6 mice and Long-Evans hooded rats [536, 566]. Similar patterns were seen with respect to $5 \mathrm{mC}$ immunoreactivity, where our mice displayed the lowest immunoreactivity in the CA3 subregion, while C57BL/6 wild-type mice showed the highest level of immunoreactivity in this subregion [567]. However, no sex- or PS-related differences could be observed within this subregion, although some tendencies for $5 \mathrm{mC}$ immunoreactivity changes were observed in the DG.

No differences in intensity of $5 \mathrm{hmC}$ immunoreactivity could be observed between the groups, even though $5 \mathrm{hmC}$ is derived from $5 \mathrm{mC}$ by ten-eleven translocation 1 (TET1) [568] and therefore comparable patterns could be expected. A similar lack of association between $5 \mathrm{mC}$ and $5 \mathrm{hmC}$ in APPswe/PS1 $\triangle \mathrm{E} 9$ mice was found in an aging study within our group (Chouliaras et al. unpublished data). Here, it was demonstrated that wild-type C57BL/6 mice were positively correlation between $5 \mathrm{mC}$ and $5 \mathrm{hmC}$ in the $\mathrm{DG}$ and $\mathrm{CA} 3$, while no such correlation could be established in APPswe/PS1 $1 \mathrm{E} 9$ mice. This lack of correlation could indicate a potentially disturbed balance between methylation and hydroxymethylation in APPswe/PS1 $1 \triangle 9$ mice. Of note, our developmental stress exposure did not modify this disturbed balance, nor did we find major sex differences in $5 \mathrm{mC}$ and $5 \mathrm{hmC}$ regulation. Evidently, genetic association between TET1 and AD has been reported [569], suggesting that a disturbance in the conversion of $5 \mathrm{mC}$ to $5 \mathrm{hmC}$ may play an important role in $A D$, along similar lines as the lack of association between $5 \mathrm{mC}$ and $5 \mathrm{hmC}$ in our mouse model. Unfortunately, very little is known yet on the role of $5 \mathrm{hmC}$ in $A D$ pathology, and many techniques frequently used for identifying the role of DNA methylation in neurodegenerative diseases cannot readily distinguish between $5 \mathrm{mC}$ and $5 \mathrm{hmC}$, probably leading to an underestimation of the importance of $5-\mathrm{hmC}$ in gene regulation [570,571]. In addition, it should be noted that our immunohistochemical approach reflects global methylation and hydroxymethylation patterns, and so effects of PS exposure on the degree of methylation and hydroxymethylation at specific sites remain to be elucidated. In that respect, this study can be viewed as a preliminary approach to explore the contribution of changes in epigenetic regulation to the PS-induced behavioral and neuropathological effects that are reported in this study. Investigation of gene-specific methylation and hydroxymethylation patterns may yield more insight into the role of epigenetic imprinting in PS research.

The fact that female APPswe/PS1 $\mathrm{E} 9$ mice display more cognitive deficits and amyloidosis than male mice of the same age, while simultaneously showing lower levels of global DNA methylation and altered APP processing, is an interesting finding. Altered DNA methylation can modify disease susceptibility. In terms of global methylation, it has been demonstrated that $A D$ cases have generally lower levels of $5 \mathrm{mC}$ and DNMT1 in the entorhinal cortex than non-demented controls [572, 573]. In addition, gene-specific methylation is also altered in $A D$ patients, as a case report indicated that in $A D$ the APP gene was hypomethylated in comparison to a healthy control subject and a patient with Pick's disease (a non-Alzheimer type of dementia) [574]. Alternatively, cleavage of APP can also modify gene transcription, as the APP intracellular domain can interact with various 
transcriptional coactivators, thereby altering transcription of APP, $\beta$-APP cleaving enzyme, glycogen synthase kinase 3 and Tip60 (a histone acetyltransferase) [575-577]. The APP intracellular domain can therefore affect gene transcription and create a vicious cycle of enhanced APP cleavage. This demonstrates that epigenetic regulation may be altered profoundly in AD. More research on the exact role of the APP intracellular domain in gene transcription could perhaps separate cause from consequence between alterations in APP processing and DNA (hydroxy)methylation. In addition, further establishment of techniques suitable to differentiate between methylation and hydroxymethylation could elucidate their precise role in the onset and progression of AD.

\subsection{Concluding remarks}

Gender differences are a well-known phenomenon in AD research, with women having a higher risk for developing AD than men [43, 504, 578]. Similarly, many AD mouse models display a sex-dependent pattern of cognitive deficits and neuropathology $[289,545,556$, $579,580]$. Surprisingly, very few studies have compared the difference in behavioral phenotype, and/or neuropathology between male and female $A D$ mice within one study design, particularly in relation to stress exposures.

The current experimental setup allowed us to directly compare the sex-specific response to PS exposure in APPswe/PS1 $1 \mathrm{E} 9$ mice. It was demonstrated that PS sexdependently altered the behavioral and neuropathological phenotype of this $A D$ mouse model. Female mice exposed to PS showed relative protection from spatial memory deficits, as well as increased depressive-like behavior and a decrease in hippocampal plaque load. PS in male mice led to an acceleration of AD-related cognitive deficits and induced a blunted HPA axis response in the face of stressors. In addition, sex differences were observed in the APPswe/PS1 $1 \mathrm{E} 9$ mouse model as female mice showed more spatial memory impairments, elevated corticosterone secretion, higher hippocampal plaque load, altered APP processing in the CA3 and lower DNMT3a immunoreactivity in the DG than male APPswe/PS1 $\triangle E 9$ mice, independent of gestational stress exposure. Since AD-related symptoms and neuropathology appear to be accelerated in female APPswe/PS1 $\triangle \mathrm{E} 9$ mice and possible pathogenic mechanisms, such as APP processing and DNA methylation, show sexual differentiation, our findings could shed a new light on the selective vulnerability of females to develop AD. Conversely, in male APPswe/PS1 $\triangle E 9$ mice, none of the investigated variables could offer a causal explanation for the observed impairment in cognitive function after PS. As cognitive deficits have been reported previously after PS exposure, also in non-AD mouse models [511], it is tempting to speculate that the cognitive impairments in male APPswe/PS1 $1 \mathrm{E} 9$ mice occur via mechanisms other than those specific to AD.

The observed sex differences should raise caution in research on AD mouse models. Preferably, researchers should use both sexes separately when investigating the effects of a certain environmental exposure in $A D$ mouse models, and make a clear comparison between the sexes. If possible, mixing male and female mice within a single experiment 
without differentiating between them should be avoided. Clearly, more research is needed to further establish the exact contribution of sexual differentiation of the brain and environmental exposure in $A D$ development, and to determine to which extent alterations in epigenetic regulation can explain or contribute to the etiopathogenesis of this disorder.

\section{Acknowledgements}

Funds have been provided by the Internationale Stichting Alzheimer Onderzoek (ISAO), grant number 09552, and the Netherlands Organization for Scientific Research (NWO, Veni Award 916.11.086) to B.P.F. Rutten, by the ISAO, grant numbers 07551 and 11532 , to D.L.A. van den Hove and by a Marie Curie Host Fellowship Grant MC-EST 020589 EURON to L. Chouliaras. 


\section{Supplementary data}

A

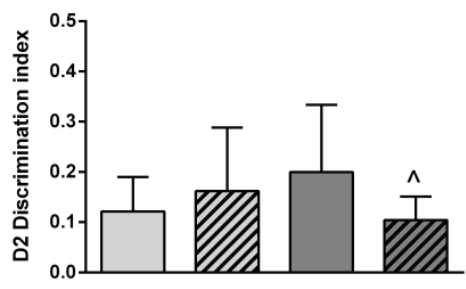

C

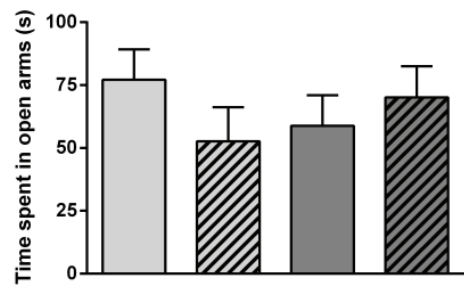

B

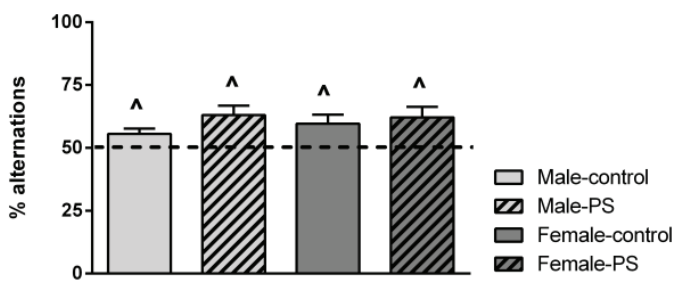

D

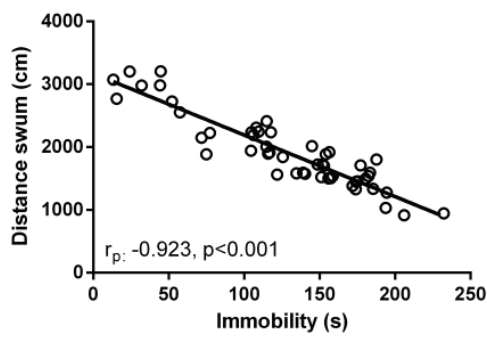

Figure S1: Supplementary data on behavioral results; bars indicate mean and S.E.M. per group (A-C), individuals points represent individual animals (D). A: Object location task $4 \mathrm{~h}$ interval. Female PS mice still significantly remember the familiar location after a $4 \mathrm{~h}$ interval. B: Y-maze spontaneous alternations test. All groups performed significantly better than $50 \%$. C: Time spent in the open arms of the elevated zero maze. No difference in anxious behavior was observed between the groups. D: Correlation analysis between immobility and distance swum. Immobility scores correlate very strongly with total distance swum. ${ }^{\wedge}: \mathrm{p}<0.05$, compared to $0(\mathrm{~A})$ or $50(\mathrm{~B})$.

A

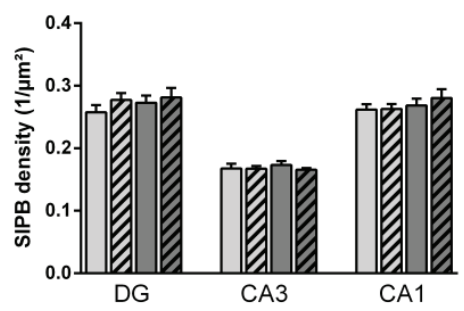

B

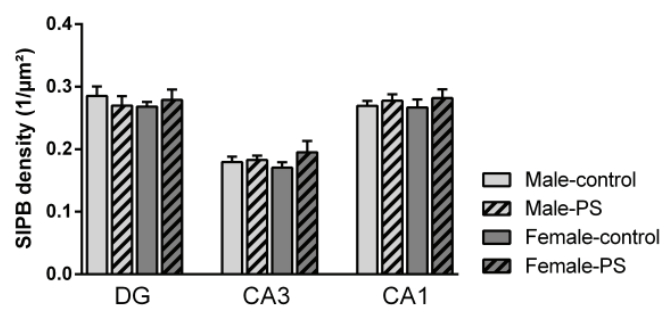

Figure S2: Analysis of synaptophysin-immunoreactive bouton (SIPB) density in the dorsal (A) and ventral (B) hippocampus. Bars indicate mean and S.E.M. per group. No difference in SIPB density can be found between the groups. 
A

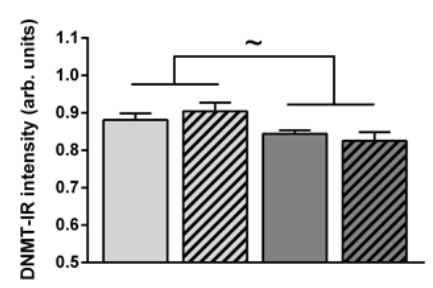

C

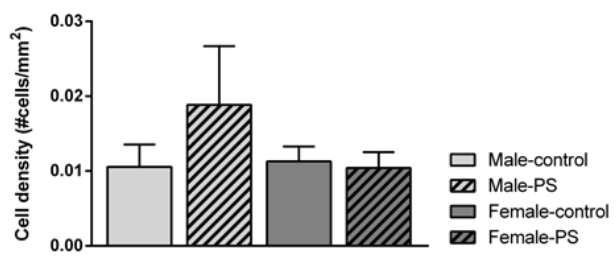

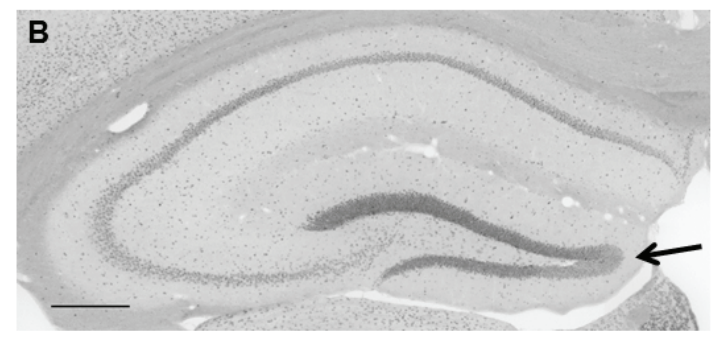

Figure S3: DNA methyltransferase 3a (DNMT3a) immunoreactivity. Bars indicate mean and S.E.M. per group. A: Intensity of DNMT3a immunoreactivity was quantified separately for the tip of the DG, and females have significantly lower immunoreactivity in the tip of the DG than male mice. B: Representative image of DNMT3a immunoreactivity in 10x where it can be noted that the tip of the DG has less immunoreactivity than the upper and lower blade; scale bar: $500 \mu \mathrm{m}$. C: Intense type II DNMT3a immunoreactive cell density of the total hippocampus. No statistical difference in cell density among the different groups can be observed within the hippocampus. : significant sex effect, $p<0.05$. 


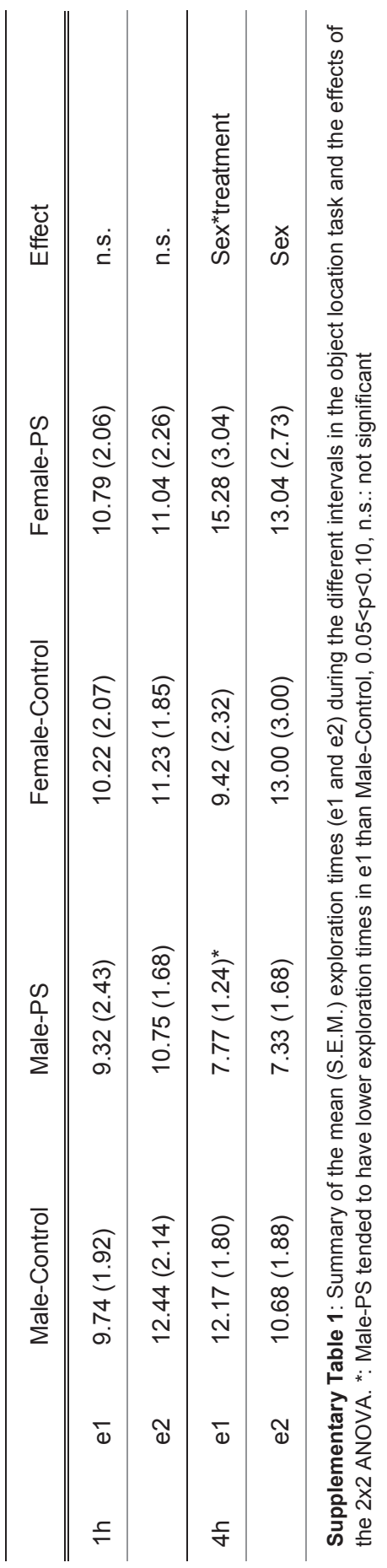




\section{Chapter 5}

Effects of prenatal stress exposure on soluble $A \beta$ and brainderived neurotrophic factor signaling in male and female APPswe/PS1 $1 \triangle \mathrm{E} 9$ mice

Annerieke SR Sierksma, Tim Vanmierlo, Jochen De Vry, Marjolein EA Raijmakers, Harry WM Steinbusch, Daniel LA van den Hove, Jos Prickaerts 


\section{Abstract}

Chronic stress and stress-related disorders, such as major depression (MD), have been shown to increase the risk for developing Alzheimer's disease (AD). Brain-derived neurotrophic factor (BDNF) has been postulated as a neurophysiological link between these illnesses. Our previous research has indicated that exposing the APPswe/PS1 $\triangle E 9$ mouse model of $A D$ to prenatal maternal stress (PS) induced a depressive-like phenotype, specifically in female mice. Considering the role of BDNF in depressive-like behavior and its interactions with amyloid- $\beta(A \beta)$, our aim was to explore whether these mice would also exhibit alterations in soluble $A \beta$, mature BDNF (mBDNF), proBDNF, and the receptors TrkB and $\mathrm{p} 75^{\mathrm{NTR}}$ in comparison to non-stressed animals. Our results demonstrate that female APPswe/PS1 $\triangle E 9$ mice have higher levels of hippocampal proBDNF and soluble $A \beta$ as compared to their male littermates. Additionally, a tendency was observed for PS to lower mBDNF protein levels in the hippocampus, but only in female mice, while receptor levels remained unaltered by sex or PS exposure. Given that female mice both have higher proBDNF and $A \beta$ levels, these findings suggest an underlying role for BDNF signaling and $A \beta$ production in the selective vulnerability of women for $M D$ and $A D$ development. 


\section{Introduction}

Epidemiological research has indicated that stress-related disorders, such as major depression (MD), can be risk factors for Alzheimer's disease (AD) development [114] and induce an earlier manifestation of clinical symptoms in the familial AD cases [75]. Indeed, patients with a history of MD are twice more likely to develop $A D$, display more hippocampal amyloid- $\beta(A \beta)$ plaques and neurofibrillary tangles, and show faster cognitive decline [114].

Decreases in brain-derived neurotrophic factor (BDNF) have been stipulated a neurophysiological commonality between $M D$ and $A D$. Indeed, BDNF mRNA levels are significantly reduced in the hippocampus of both $M D$ and $A D$ patients compared to controls and both patient groups suffer from cognitive dysfunction [115]. BDNF, known to promote plasticity, proliferation, survival and differentiation, is widely expressed in the hippocampus and closely linked to learning and memory processes. Its precursor proBDNF consists of a pro-domain and a mature domain, and can be proteolytically cleaved into mature BDNF (mBDNF) and different isoforms of proBDNF, which is also subject to glycosylation. mBDNF preferentially binds to the tropomyosin-related kinase $B(T r k B)$ receptor, thereby exerting the neurotrophic effects often attributed to BDNF. ProBDNF, on the other hand, preferentially binds to the p75 neurotrophin receptor $\left(p 75^{\mathrm{NTR}}\right)$, and is thereby able to activate the proapoptotic c-Jun N-terminal kinase signaling pathway [581].

The observation that depressed suicide victims have decreased BDNF protein expression and that BDNF upregulation is necessary and sufficient for effective antidepressant treatment, has led to the formulation of the neurotrophic hypothesis of depression. This proposes that lower neurotrophic support mediates the hippocampal atrophy, cognitive dysfunction and decrease in neuronal plasticity often seen in MD patients [406]. Considering BDNF's role in memory formation and cognitive function, and the fact that these functions are also comprised in $A D$, it should be noted that alterations in neurotrophic support have also been reported abundantly for $A D$ patients, including reductions in hippocampal BDNF expression levels [115]. In addition, decreases in the level of TrkB have been consistently reported in $A D$ patients, whereas the literature about $p 75^{\mathrm{NTR}}$ alterations remains conflicting, despite its abundant presence in the cholinergic forebrain neurons, which are the first to degenerate in $\operatorname{AD}[115,582]$. Moreover, significant associations have been found between BDNF gene single nucleotide polymorphisms (SNPs), i.e. C270T and A196G, AD, and also AD-related depression [115]. Of note, meta-analysis on these associations revealed that only women were at increased risk, not men [583].

As $A \beta$ can serve as a ligand for the $p 75^{\mathrm{NTR}}$ receptor, it has been suggested that this receptor can at least partly mediate $A \beta$ 's neurotoxicity [582]. Alternatively, binding of BDNF to the TrkB receptor can alter $A \beta$ production by influencing amyloid precursor protein (APP) promoter activity, APP processing and $\beta$-amyloid cleaving enzyme 1 (BACE1) activity [584, 585]. Vice versa, $A \beta$ can also affect BDNF expression and signaling by inhibiting cAMP response element-binding (CREB)-induced BDNF transcription and retrograde trafficking of BDNF/TrkB complexes [115, 586]. 
Based on the above, alterations in neurotrophic signaling could help explain the MD-AD interaction found in epidemiological data. Markedly, women are more at risk for the development of both $M D$ and $A D$ [130] and preclinical studies indicate distinct sexdependent alterations in hippocampal BDNF concentrations after stress exposure $[553,587-$ 589], We therefore aimed to investigate the role of the BDNF signaling pathway in the link between depressive-like behavior and the progression of an AD-like phenotype, and whether this role would be sex-specific. To that extent, we previously exposed the APPswe/PS1 $\triangle E 9$ mouse model of $A D$ to prenatal maternal stress (PS), which is known to induce MD-like symptoms in adult offspring [509], to investigate the effects on behavior and $A \beta$ plaque load. Our results indicated that PS was able to induce a depressive-like phenotype with concomitant alterations in plaque load, specifically in female mice. In male mice, PS induced spatial memory deficits, without affecting depressive-like behavior [590]. The aim of the current study was to investigate whether soluble $A \beta 42$ and $A \beta 40$ levels, and their ratio, would be affected in a similar fashion as plaque load, showing a sex-specific effect of PS exposure. Additionally, we wished to explore whether the sex-specific alterations in behavioral and neuropathological phenotype as a result of PS could be explained by alterations in mBDNF, proBDNF and the TrkB and $\mathrm{p} 75^{\mathrm{NTR}}$ receptors, as the BDNF signaling pathway is thought to play a vital role in depressive-like behavior, neuroplasticity and AD.

\section{Material \& Methods}

\subsection{Breeding and prenatal stress procedure}

All animals were housed under a $12 \mathrm{~h}$ light/dark cycle (lights on at 7:00 A.M) with an average temperature of $22^{\circ} \mathrm{C}$ and relative humidity of $42 \%$. Food and water were provided ad libitum throughout the study. All studies were executed according to protocols approved by the Animal Ethical Committee of Maastricht University, Maastricht, The Netherlands and met governmental guidelines (UM-DEC 2008-004).

To obtain offspring, virgin female C57BL/6NCrl mice (Charles River, L'Arbresle, France) were mated with male APPswe/PS1 $\triangle E 9$ mice (Jackson Laboratory, strain \#005864) at 8-12 weeks of age, as described previously [590]. Presence of a copulation plug was considered embryonic day 0 (E0) and, subsequently the females were housed individually, given tissue for nest building, and assigned to a treatment group.

The prenatal stress paradigm was performed as described previously [510, 590]. In short, pregnant females assigned to the prenatal stress (PS) group were subjected to chronic restraint stress by placing the mouse in a $5 \mathrm{~cm}$ diameter glass cylinder, filling the cylinder with water to a height of $1 \mathrm{~cm}$ and positioning them underneath a bright light. Restraint stress was performed 3 times per day (at approximately 8:00h; 12:00h; 16:00h), for 45 min per session from E1-E7. After each restraint stress session, the animals were placed back into their home cage. Control mice were left undisturbed in their home cage. 


\subsection{Offspring}

At postnatal day (P) 25 all animals were ear-punched and marked to enable permanent identification while harvesting tissue for genotyping using PCR amplification of genomic DNA, as described in [590]. At P30 ( \pm 1 day), APPswe/PS1 $\Delta$ E9 pups were weaned and housed per 2 with same sex littermates. Four experimental groups were formed: malecontrol $(n=11)$, male-PS $(n=8)$, female-control $(n=11)$, female-PS $(n=5)$. Of note, these mice were littermates of mice used in [590].

\subsection{Tissue preparation}

At 8 months all mice were sacrificed by decapitation, and the whole hippocampus was isolated. Hippocampal tissue was snap frozen in liquid nitrogen and homogenized using a Mini-Bead Beater (Biospec products, Bartlesville, USA) two times for 30 seconds in $2 \mathrm{ml}$ icecold lysis buffer (137mM NaCl, 20mM Tris- $\mathrm{HCl}(\mathrm{pH} 8.0), 1 \%$ Igepal, $10 \%$ glycerol and one complete protease inhibitor tablet (Roche \#11836153001, Vilvoorde, The Netherlands). Samples were centrifuged for five minutes at $4^{\circ} \mathrm{C}, 1000 \mathrm{RCF}$, and the supernatant was stored at $-80^{\circ} \mathrm{C}$. Protein concentrations were determined using the Bio-Rad Lowry Protein Assay (Bio-Rad Laboratories Inc., Hercules, USA).

\subsection{ELISA AB40+42}

Concentrations of hippocampal $A \beta 40$ and $A \beta 42$ were determined in the hippocampal homogenates using ELISA kits against human A 440 and $A \beta 42$ (Invitrogen, \#KHB3481 and \#KHB3544, Camarillo, USA), following the manufacturer's protocol. In addition, the $A \beta 42 / A \beta 40$ ratio was determined for each group.

\subsection{Western Blotting}

Brain homogenates in sample buffer were boiled for 5 minutes and then separated on a $10 \%$

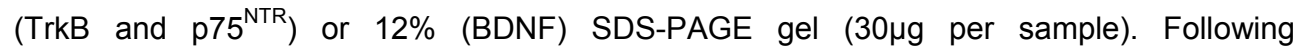
electrophoresis, proteins were transferred to a nitrocellulose membrane (Bio-Rad Laboratories, Hercules, USA) which was previously blocked with blocking buffer $(50 \%$ Odyssey blocking buffer in PBS, Li-Cor, Lincoln, USA) for one hour at room temperature. Next, the membranes were incubated overnight at $4^{\circ} \mathrm{C}$ with the primary antibodies in blocking buffer: 1:600 rabbit anti-BDNF ( $\mathrm{H}-117)$ (Santa Cruz Biotechnology \#SC-20981, Heidelberg, Germany), 1:1000 rabbit anti-TrkB (Cell Signaling Technology \#4606, Beverly, USA), 1:1000 rabbit anti-p75 ${ }^{\mathrm{NTR}}$ (Millipore \#07-476, Billerica, MA) with 1:2.000.000 mouse anti-GAPDH (\#10R-G109A, Fitzgerald, Huissen, The Netherlands) for normalization. After washing with phosphate-buffered saline- $0.1 \%$ Tween (PBS-T), membranes were incubated for 1 hour at room temperature with secondary antibodies in blocking buffer: 1:5000 goat anti-rabbit IRDye 800 (\#926-32211, Li-Cor) and 1:5000 donkey anti-mouse IRDye 680 (\#926-32222, Li-Cor). Membranes were washed in PBS-T and fluorescent bands were visualized using an Odyssey Infrared Imaging System (Li-Cor). Intensities of specific bands 
were quantified using ImageJ (http://rsbweb.nih.gov/ij/), corrected for background signal and GAPDH signal. Within the BDNF blot different BDNF domains were analyzed, distinguishing between mBDNF (14 kDa) and two forms of proBDNF namely, a glycosylated form (18 kDa) and a unglycosylated form (16 kDa), as previously described [591-593]. Ratios between mBDNF/unglycosylated proBDNF and mBDNF/glycosylated proBDNF were also determined.

\subsection{Statistical analysis}

All data are presented as mean and standard error of the mean (SEM). Two-way ANOVA was performed for comparison between groups accounting for the main and interactive effects of sex (male, female) and stress exposure (control, PS). As clear sex effects were expected based on previous work [590], additional student's t-tests were performed comparing the PS and control groups for each sex separately. Statistical significance was set at an a level of 0.05 and all tests were two-tailed. All statistical analyses were conducted using the Statistical Package for the Social Sciences (SPSS 15.0, SPSS Inc., Chicago, IL, USA). All graphs were constructed in GraphPad Prism (version 4, GraphPad Software, San Diego, CA, USA).

\section{Results}

\subsection{Soluble amyloid- $\beta$}

Analysis of the soluble fractions of $A \beta 40$ and $A \beta 42$ revealed significant sex differences $((F(1,37)=17.06, p<0.001), \quad(F(1,38)=10.17, p<0.01)$, respectively $)$, with females having higher concentrations of hippocampal $A \beta 40$ and $A \beta 42$. PS exposure alone or the interaction of sex*stress exposure did not affect $A \beta 40$ or $A \beta 42$ concentrations. Analysis of the $A \beta 42 / A \beta 40$ ratio indicated a significant sex effect $(F(1,37)=6.55, p<0.05)$, with female mice having a lower ratio. Stress exposure or sex*stress exposure did not influence the $A \beta 42 / A \beta 40$ ratio (see Fig.1A-C). 
A

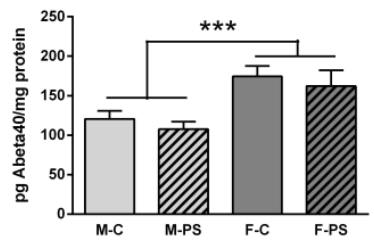

B

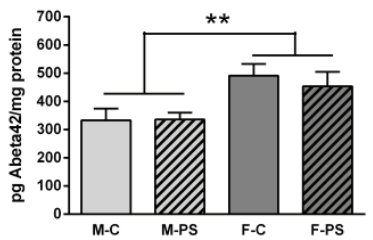

C

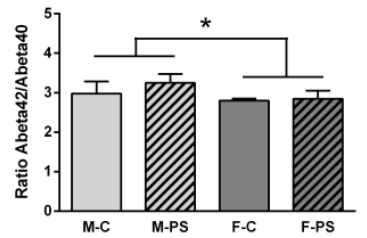

Figure 1: Female APPswe/PS1 $\triangle E 9$ mice have significantly more soluble hippocampal $A \beta 40(A)$ and $A \beta 42(B)$ as compared to male mice. Conversely, females had a lower $A \beta 42 / A \beta 40$ ratio than male mice (C). Bars indicate mean and SEM per group. M-C: male-control; M-PS: male-prenatal stress; F-C: female-control; F-PS: female-prenatal stress; ${ }^{* * *}: p<0.001$; ${ }^{* *} p<0.01 ;{ }^{*}: p<0.05$.

\subsection{Western blotting}

\subsection{1. $m B D N F$ and proBDNF}

Analysis of the hippocampal mBDNF concentrations demonstrated a trend for a sex ${ }^{*}$ stress exposure effect $(F(1,33)=2.99, p=0.094)$, but no main effects of sex or stress exposure. The student's t-test revealed a significant decrease in hippocampal mBDNF in the female-PS group, compared to female-controls $(\mathrm{t}(11.52)=2.57, \mathrm{p}<0.05)$. Both unglycosylated and glycosylated proBDNF were significantly affected by sex $((F(1,33)=7.11, p<0.05), \quad(F(1$, $33)=10.98, p<0.01$ ), respectively), with female mice having more proBDNF. Stress exposure and the interaction of sex*stress exposure did not affect unglycosylated and glycosylated proBDNF concentrations in the hippocampus. The ratio between mBDNF/unglycosylated proBDNF and $\mathrm{mBDNF} / \mathrm{glycosylated}$ proBDNF was also affected significantly by sex $((\mathrm{F}(1$, $33)=5.65, p<0.05),(F(1,33)=9.61, p<0.01)$, respectively $)$, with females having lower ratios as compared to male mice. The ratios were not affected by stress exposure or sex*stress exposure (see Fig. 2A-F).

\subsubsection{TrkB and $p 75^{N T R}$}

No effects of sex, treatment or sex*treatment were found on the concentration of the BDNF receptors TrkB or $\mathrm{p} 75^{\mathrm{NTR}}$ (see Figure $2 \mathrm{G}-\mathrm{H}$ ). 
A

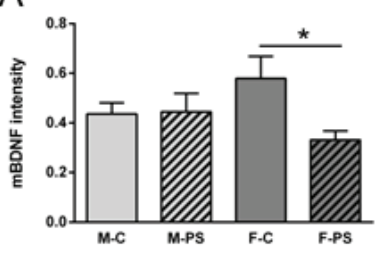

C

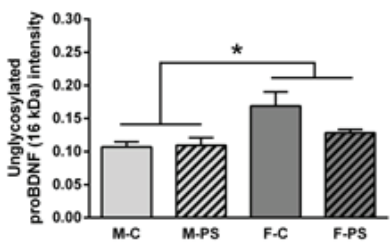

$\mathrm{E}$

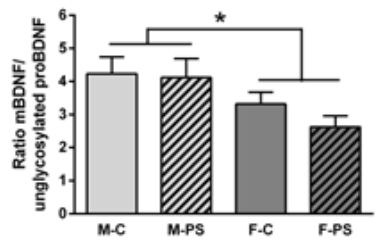

G

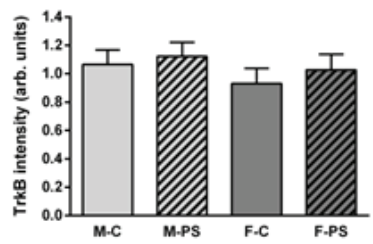

B $\quad M-C \quad M-P S \quad F-C \quad$ F-PS

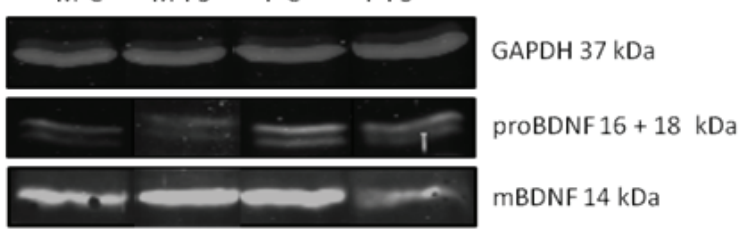

D

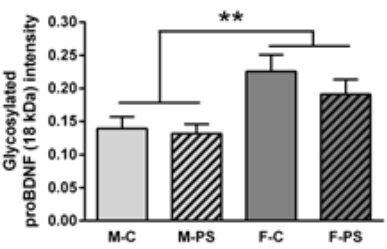

$\mathrm{F}$

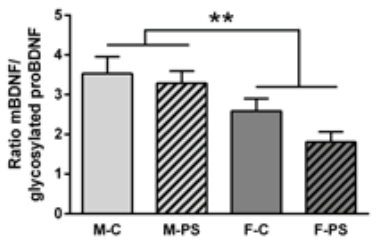

$\mathrm{H}$

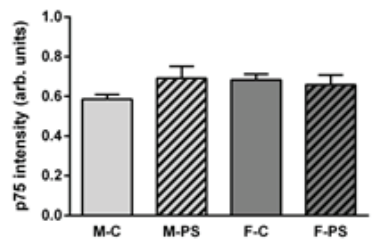

Figure 2: Protein levels of mature BDNF (mBDNF), unglycosylated and glycosylated proBDNF, TrkB and p75NTR in the hippocampus. Bars indicate mean and SEM per group. A: mBDNF concentrations are significantly reduced after prenatal stress (PS), but only in female APPswe/PS1 $\triangle E 9$ mice. B: representative immunoreactive bands from BDNF western blot. C, D: Female mice generally displayed higher unglycosylated proBDNF (16 kDa) and unglycosylated proBDNF (18 kDa) concentrations than male mice. $\mathrm{E}, \mathrm{F}$ : The ratio between $\mathrm{mBDNF} /$ unglycosylated proBDNF (E) and $\mathrm{mBDNF} / \mathrm{glycosylated}$ proBDNF $(F)$ was significantly lower in female mice compared to male mice. $G, H$ : No differences in TrkB $(G)$ or p75NTR $(H)$ levels are observed between the groups. M-C: male-control; M-PS: male-PS; F-C: female-control; F-PS: female-PS; ${ }^{*}: p<0.05 ;{ }^{* *}: p<0.01$. 


\section{Discussion}

In this study, we observed that adult female APPswe/PS1 $\triangle \mathrm{E} 9$ mice have much higher levels of soluble $A \beta 40$ and $A \beta 42$ in the hippocampus compared to male mice of the same age. This is in line with previous reports of higher plaque load in female mice [590]. Moreover, female APPswe/PS1 $\triangle E 9$ mice exhibited a lower $A \beta 42 / A \beta 40$ ratio than male mice, reflecting the proneness of $A \beta 42$ to aggregate into plaques and not remaining in a soluble fraction. However, PS exposure did not alter soluble $A \beta 40$ or $A \beta 42$ levels in either of the sexes, even though a decreased dorsal plaque load in female APPswe/PS1 1 E9 mice after PS was demonstrated previously [590]. This discrepancy could possibly be explained by the fact that the decreased plaque load was found only in the dorsal hippocampus, while our homogenates contained the whole hippocampus, thereby obscuring these sex-specific PS effects.

Additionally, it was demonstrated that the ratio of neuroprotective mBDNF versus proapoptotic proBDNF was significantly lower in female mice as compared to their male counterparts, suggestive of a distinct (patho)physiological role of proBDNF in female APPswe/PS1 $1 \triangle \mathrm{E} 9$ mice. In this light, it is worth mentioning that female APPswe/PS1 $\triangle E 9$ mice also display more spatial memory deficits as compared to male mice of the same age [590]. Considering the role of proBDNF in memory extinction and in long-term depression facilitation [581], this selective increase in proBDNF may actively contribute to the cognitive impairments seen in female mice. Of note, estrogen is co-expressed with BDNF in many brain areas, such as the hippocampus, and the BDNF gene has an estrogen-sensitive response element, which enables estrogen to induce BDNF mRNA and protein expression. Moreover, BDNF and estrogen exert similar effects on behavior, cellular structure and function, and can alter the same intracellular cascades such as MAP/ERK and PI3K/Akt [595], making it likely that the BDNF system is differentially regulated among males and females. Along similar lines, various studies do indicate distinct sex-dependent hippocampal alterations in BDNF concentrations after stress exposure [553, 587-589].

Further, the data from this study suggests that female APPswe/PS1 $\triangle E 9$ mice exposed to PS have lower levels of hippocampal mBDNF than control female APPswe/PS1 $1 \mathrm{E} 9$ mice, although the low number of samples decreased the statistical power. Our previous work showed that only female APPswe/PS1 $\triangle \mathrm{E} 9$ mice displayed more depressive-like behavior in the forced swim test after PS exposure [590]. Lower hippocampal BDNF concentrations have been associated with MD and depressive-like behavior [406], while higher levels of mBDNF were linked to reductions in depressive-like behavior [594]. In this regard, previous research has indicated that in face of lower mBDNF levels, female mice are more prone to develop a MD-related phenotype [596]. By exposing a forebrain inducible BDNF knockout model to chronic unpredictable stress, more depressive-like behavior was induced only in female mice [596]. With the neurotrophic hypothesis of depression in mind, it could therefore be speculated that the increase in depressive-like behavior in female PS mice is mediated 
via lower mBDNF levels and the consequent reduction in neurotrophic support [406], although these results do need further verification.

Mature BDNF and proBDNF levels differed between the investigated groups, while the level of TrkB and $\mathrm{p} 75^{\mathrm{NTR}}$ receptors in the hippocampus remained unaltered. Additionally, TrkB's truncated form, TrkB.T1, which can serve as a dominant negative inhibitor of BDNF signaling, was not altered by sex or PS (data not shown). Despite the observation that $A \beta$ is able to increase TrkB.T1 and $\mathrm{p} 75^{\mathrm{NTR}}$ and down-regulate the full length TrkB receptor expression $[597,598]$ and our female mice displayed higher levels of $A \beta$ than male mice, the amount of hippocampal BDNF receptors in our research remained unchanged. Notably, the p75 receptor is abundantly expressed in the cholinergic basal forebrain neurons, which degenerate early in the AD process [582]. Yet, as others suggest that cholinergic innervation is not affected at 7 months of age in the APPswe/PS1 $\triangle$ E9 mouse model [599], it is unlikely that these forebrain neurons have degenerated yet in our mice. This might explain why expression of the p75 receptors remained unaltered as a consequence of PS. It appears that possible differences in neuroprotective or neurodegenerative effects between our experimental groups are not linked to changes in receptor levels, but are more likely mediated via differences in ligand availability.

The $p 75^{\mathrm{NTR}}$ receptor can bind both proBDNF and $A \beta$ levels $[581,582]$, both of which are elevated in female as compared to male mice. As we did not observe differences in depressive-like behavior between the sexes per se, it is unlikely that proBDNF and soluble $A \beta$ have a direct influence on depressive-like behavior, although they may explain the impaired spatial memory performance in female compared to male mice [590].

In conclusion, BDNF signaling and amyloid production appear to be sex-dependent, as female APPswe/PS1 $\triangle E 9$ mice exhibit higher levels of soluble $A \beta 40$ and $A \beta 42$ as well as proBDNF. This is in line with epidemiological data stating that AD-associated BDNF SNPs only convey their risk onto women [583]. Overall, these findings may shed a new light on the selective vulnerability of women to develop both $M D$ and $A D$, and propose alterations in BDNF signaling and $A \beta$ production as underlying mechanisms.

\section{Acknowledgements}

This study was partly supported by grants from the Internationale Stichting Alzheimer Onderzoek (ISAO; grant number 07551 and 11532) to Dr. Daniel van den Hove. 


\section{Chapter 6}

Chronic phosphodiesterase type 2 inhibition improves memory in the APPswe/PS1 $\triangle \mathrm{E} 9$ mouse model of Alzheimer's disease

Annerieke SR Sierksma, Kris Rutten, Sebastian Sydlik, Somayeh Rostamian, Harry WM Steinbusch, Daniel LA van den Hove, Jos Prickaerts

Neuropharmacology (Special Issue: Cognitive Enhancers; 2013), 64(1), p.124-136 


\section{Abstract}

Alzheimer's disease (AD) is characterized by progressive cognitive deficits and synaptic dysfunction. Over the last decade phosphodiesterase inhibitors (PDE-Is) have received increasing attention as putative cognition enhancers and have been suggested as a novel treatment strategy for AD. Given their ability to prevent hydrolysis of CAMP and/or cGMP, they can stimulate the CAMP/protein kinase A (PKA)/CAMP element-binding protein (CREB) and CGMP/PKG/CREB pathway to enhance synaptic transmission by increasing CREB phosphorylation ( $\mathrm{pCREB}$ ) and brain-derived neurotrophic factor (BDNF) transcription. Based on previous research, we hypothesized that chronic PDE2-I treatment would improve ADrelated cognitive deficits, by decreasing amyloid- $\beta$ (A $\beta$ ) plaque load, enhancing PCREB and BDNF levels and increasing synaptic density in the hippocampus of 8-month-old APPswe/PS1 $\triangle E 9$ mice. Results indicated that chronic PDE2-I treatment could indeed improve memory performance in APPswe/PS1 $\triangle E 9$ mice, without affecting anxiety, depressive-like behavior or hypothalamus-pituitary-adrenal axis regulation. However, no treatment effects were observed on A $\beta$ plaque load, pCREB or BDNF concentrations, or presynaptic density in the hippocampus, suggesting that other signaling pathways and/or effector molecules might be responsible for its cognition-enhancing effects. Presynaptic density in the stratum lucidum of the CA3 subregion was significantly higher in APPswe/PS1 $\triangle E 9$ mice compared to WT controls, possibly reflecting a compensatory mechanism. In conclusion, PDEs in general, and PDE2 specifically, could be considered as promising therapeutic targets for cognition enhancement in $A D$, although the underlying mechanism of action remains to be elucidated. 


\section{Introduction}

Alzheimer's disease (AD) is a neurodegenerative disorder, initially characterized by memory loss and followed by functional impairment in a multitude of cognitive domains. On a neuropathological level, patients suffer from progressive amyloid- $\beta(A \beta)$ plaque deposition, neurofibrillary tangle formation and synaptic dysfunction in brain regions involved in learning, memory and other higher cognitive functions. As synapse loss correlates more robustly with cognitive deficits than any other AD hallmark [25], it is thought that synaptic dysfunction underlies the cognitive deficits observed in $A D$ patients.

Phosphodiesterase (PDE) inhibitors (PDE-Is) are receiving increased attention as putative cognition enhancers [55]. PDE-Is prevent the selective breakdown of the cyclic nucleotides cyclic adenosine monophosphate (cAMP) and/or cyclic guanosine monophosphate (cGMP) by inhibiting PDE enzymes. PDEs can be classified into 11 families, and can either hydrolyze both cyclic nucleotides, e.g. PDE2, or are specific for a single nucleotide, e.g. PDE4 specifically hydrolyzes cAMP, while PDE5 selectively targets cGMP [56]. The second messenger cAMP, synthesized from ATP by adenylate cylcase $(A C)$, in its turn can activate protein kinase $A$ (PKA) which phosphorylates the cAMP response element-binding protein (CREB), and can thereby affect transcription of genes related to synaptic plasticity and survival, like brain-derived neurotrophic factor (BDNF) [57]. Conversely, cGMP is derived from GTP by guanylyl cyclase (GC), which in turn is activated by nitric oxide (NO). The NO/cGMP pathway then activates protein kinase G (PKG), which can also induce CREB phosphorylation [58]. Both the CAMP/PKA/CREB and the cGMP/PKG/CREB pathway are implicated in long-term potentiation (LTP), the neurophysiological correlate of memory [58-60].

Acute and/or chronic administrations of specific PDE-Is, including PDE2, 4 or 5 inhibitors, in rodents, macaques and humans has been shown to improve various learning and memory processes, attention, cognitive flexibility, information processing, and executive functioning [600] (for an extensive overview, see [55]), of which many processes are impaired in AD. On a cellular level, PDE4 and PDE5 inhibition can augment synaptic transmission by facilitating LTP and thereby possibly contributing to memory formation [66, 67, 601-603].

It has been shown that $A \beta$ can have a detrimental effect on LTP, via inhibition of both the cAMP/PKA/CREB and cGMP/PKG/CREB pathway [61, 70, 604]. In addition, AC, GC and pCREB levels are reduced in the temporal lobe of $A D$ patients [605-608]. Given the cognition-enhancing properties of PDE-Is in rodents and monkeys and the synaptic dysfunction and cognitive deficits seen in AD patients, PDE-Is are currently in development as new therapeutics for the treatment of $A D[55,609]$. Indeed, acute and treatment with the PDE4I rolipram has shown to improve memory function in mouse models of $A D$, restore pCREB levels to wild-type (WT) levels, yet without affecting $A \beta$ levels [65, 66]. Similarly, chronic administration of the PDE5I sildenafil could restore AD-related cognitive deficits, synaptic dysfunction and pCREB levels, but also decreased hippocampal A $\beta$ levels [67]. 
Intrahippocampal injection of $A \beta$ peptides in rats led to similar memory and $\mathrm{pCREB}$ alterations as observed in $A D$ mouse models, which could again be reversed by chronic rolipram treatment $[610,611]$.

Despite these promising results, current PDE4Is and PDE5Is are not optimal candidates for the treatment of cognitive deficits in AD. Current PDE4ls have emetic properties, making these drugs less suitable for clinical development [68, 612]. In addition, PDE5I treatment did not induce cognition-enhancing effects in aged rats [613], the amount of NO synthase (NOS) producing neurons decreases with age [614], and PDE5 mRNA could not be detected in the cortex and hippocampus of elderly control and AD cases [69], indicating that PDE5 may not be an optimal target for memory enhancement in aged or AD patients. PDE2-I treatment, on the other hand, prevents the hydrolysis of both cAMP and CGMP, thereby targeting a wider array of intracellular pathways involved in signal transduction. The PDE2-I BAY60-7550 has already shown cognition-enhancing efficacy in mice as well as adult and aged rats [613, 615]. Moreover, PDE2 has a favorable mRNA expression pattern in the brain, even in aged individuals, with high expression levels in the hippocampus, frontal, temporal and parietal cortex [69, 616], i.e. areas associated with learning and memory processes and other higher cognitive functions. PDE2-I treatment could therefore be considered a suitable option for cognition enhancement in AD patients.

Based on the studies performed by Puzzo et al. [67] and Gong et al. [66], we hypothesized that chronic PDE2-I treatment with BAY60-7550 would similarly reverse ADrelated memory deficits, by restoring $p C R E B$ levels, enhancing expression of its downstream target BDNF, reducing $A \beta$ levels and increasing synaptic density. In addition, the effect of the treatment on other brain functions such as anxiety and depressive-like behavior was explored. Considering the expression of PDE2 in the hypothalamus and pituitary gland [616, 617], the effects of chronic BAY60-7550 treatment on hypothalamus-pituitary-adrenal (HPA) axis regulation was also examined.

\section{Material and Methods}

\subsection{Animals}

All animal experiments were approved by the ethical committee of Maastricht University for animal experiments (DEC2008-033) and met governmental guidelines. Five-month-old male C57BL/6 (WT, n=30) and APPswe/PS1 $\triangle \mathrm{E} 9$ transgenic $(\mathrm{n}=28)$ were purchased from Charles River (L'Arbresle, France). The latter express mouse/human chimeric APP695 harboring the Swedish K694M/N595L mutation and the PS1 gene with a deletion of exon 9 (PS1 $\Delta \mathrm{E} 9$ ) under the mouse prion protein promoter [47], and were backcrossed to a C57BL/6 background for at least 8 generations. This mouse model displays $A \beta$ plaques from about 4 months of age and shows the first hippocampal functional deficits from 7 months of age onwards [50-52]. The animals were housed individually in standard Makrolon cages on 
sawdust bedding, placed in an individually ventilated cage (IVC) unit, regulating temperature (approximately $20^{\circ} \mathrm{C}$ ) and humidity (between $40-60 \%$ ). A radio, which was playing softly, provided background noise in all rooms. The animals were kept under a reversed 12/12-hour light/dark cycle (lights on from 19.00 to $7.00 \mathrm{~h}$ ) and had free access to food and water. All behavioral testing was performed between 8.00 and $18.00 \mathrm{~h}$, and occurred in the room adjacent to the housing facilities. Animals were weighed at regular intervals to monitor general wellbeing. At the start of the injection period, animals were weighed approximately every 3 days, to monitor any weight changes due to the daily injections.

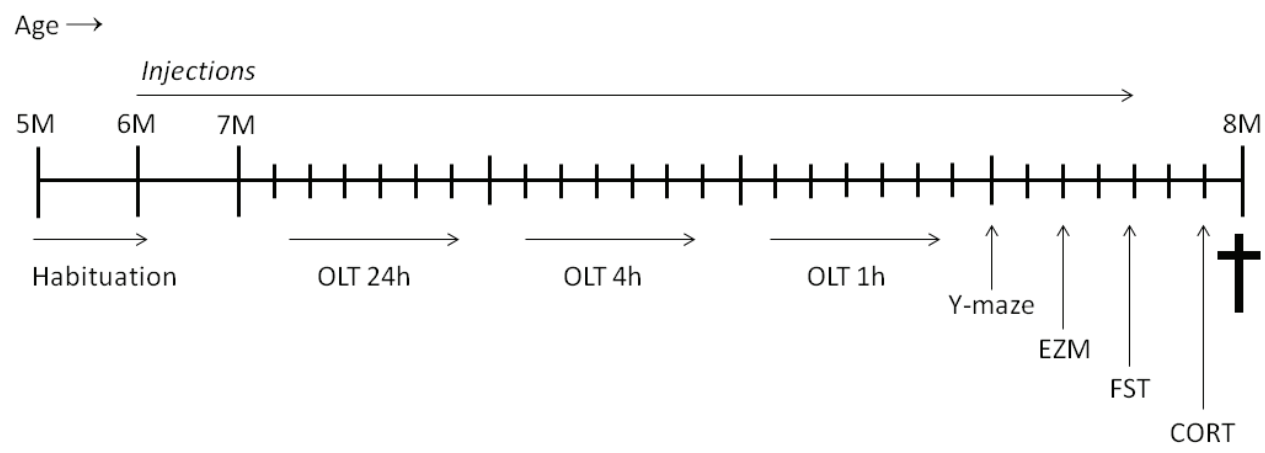

Figure 1: Timeline of the experiment expressed in months of age $(M)$ of the mice. Habituation of the mice entailed handling, weighing and habituation to the arena of the object location task (OLT). Injections, vehicle or PDE2-I, were given from 6-8M. After 4 weeks of injections, mice were subjected to the $24 \mathrm{~h}, 4 \mathrm{~h}$ and $1 \mathrm{~h}$ interval of the OLT, followed by the $\mathrm{Y}$-maze, elevated zero maze (EZM), forced swim test (FST), blood collection for corticosterone measurements (CORT), and sacrificed at 8M. The animals received their last injection at the end of the day on which the FST was performed, 3 days before their sacrifice.

\subsection{Drug administration}

Four experimental groups were generated by random group allocation: WT mice treated with vehicle (WT-veh, $n=15$ ), WT mice treated with the PDE2-I BAY60-7550 (WT-PDE, n=17), APPswe/PS1 $\triangle E 9$ mice treated with vehicle (AD-veh, $n=13$ ), and APPswe/PS1 $\triangle E 9$ mice treated with BAY60-7550 (AD-PDE, $n=13$ ). Vehicle treatment consisted of vehicle $(0.5 \%$ methylcellulose and 2\% Tween80) and BAY60-7550 was administered at $0.3 \mathrm{mg} / \mathrm{kg}$ suspended in vehicle. To prevent possible overdosing due to chronic treatment we selected the lowest effective acute dose of BAY60-7550 on object recognition memory in mice (Boess et al., 2004). All solutions were freshly prepared on a daily basis. Vehicle or BAY60-7550 were administered per oral gavage (p.o.) in a volume of $5 \mathrm{ml} / \mathrm{kg}$ at the end of each day (approximately $17.00 \mathrm{~h}$ ), for a period of 8 weeks. After 4 weeks of daily injections, behavioral testing commenced (see Figure 1 for a general timeline). The last injection was given 3 days 
before sacrificing the animals, on the same day as the forced swim test experiment. To prevent any acute effects of BAY60-7550 treatment on memory, mice did not receive a daily injection during the $24 \mathrm{~h}$ interval of the object location task (OLT) (see section 2.3.1.).

\subsection{Behavioral testing}

\subsubsection{Object location task}

The OLT was conducted as previously described [522, 523]. The apparatus consisted of a circular arena, $43 \mathrm{~cm}$ in diameter. The $40 \mathrm{~cm}$ high wall was divided into two halves, one made of white polyvinyl chloride, the other made of transparent polyvinyl chloride. Two objects were placed symmetrically in the centre of the arena. Four different sets of objects were used, and each object was available in triplicate. The different objects were: 1) a cone made of brass (maximal diameter $6 \mathrm{~cm}$ and total height $3.8 \mathrm{~cm}$ ), 2) a transparent $50 \mathrm{ml}$ tube (maximal diameter $3.5 \mathrm{~cm}$ and total height $11.5 \mathrm{~cm}$ ) filled with water, 3) a massive metal cube $(2.5 \times 5 \times 7.5 \mathrm{~cm})$ with two holes (diameter $1.5 \mathrm{~cm}$ ), and 4) a massive aluminum cube with a tapering top $(4.5 \times 4.5 \times 8.5 \mathrm{~cm})$. The objects could not be displaced by the mouse. A testing session consisted of two trials, with each trial lasting 4 minutes. During the first trial (T1) the arena contained two identical objects. A mouse was introduced into the arena by placing it with its nose to the middle of the transparent segment of the wall. After the first exploration period elapsed, the mouse was placed back in its home cage. Subsequently, after a predetermined time interval (e.g. $1 \mathrm{~h}, 4 \mathrm{~h}$, or $24 \mathrm{~h}$ ) the mouse was put back into the arena for the second trial (T2). However, this time one of the objects was relocated to a novel location. The time spent exploring the objects during T1 and T2 was recorded manually by an experienced observer using a computer. In order to determine whether a mouse has remembered the familiar location of the object, and can therefore distinguish the familiar from the novel location, several parameters are used. The amount of exploration time is recorded in T1 (named e1) and T2 (e2). The discrimination index d2, an indication of the learning process, calculates whether the mouse spends more time with the object in the novel location than with the object in the familiar location, while correcting for the total e2. Exploratory behavior was defined as follows: directing its nose to the object at a distance of no more than $1 \mathrm{~cm}$ and/or touching the object with its nose. Sitting on the object was not considered exploration. In order to avoid the presence of olfactory cues, all objects were cleaned thoroughly with ethanol and water between sessions. The order of the objects, which object was moved and to which position were all balanced out throughout the experiment and between groups, to reduce potential bias toward particular objects, sides or locations.

\subsubsection{Y-maze}

Spatial working memory was assessed by spontaneous alternation behavior in the Y-maze and was performed as previously described [284]. The symmetrical Y-maze made of acrylic 
consists of three arms, each arm being $40 \mathrm{~cm}$ long, $17 \mathrm{~cm}$ high, $4 \mathrm{~cm}$ wide at the bottom and $13 \mathrm{~cm}$ wide at the top. Each mouse was placed in the centre of the $Y$-maze and was free to explore the arena for 6 minutes. After each session the maze was thoroughly cleaned using ethanol and water and dried. The number of entries was recorded manually for each mouse, while observing the mouse via a camera; one entry meaning that both hind paws of the animal were placed completely inside the arm. A triad was scored whenever a mouse visited all three arms consecutively. The measure for working memory is the percentage of alternations, i.e. the number of triads divided by the maximum possible alternations (the total number of entries minus 2) $\times 100$. Alternation performance above chance level (i.e. $50 \%$ ) is indicative of functional spatial working memory [524].

\subsubsection{Elevated zero maze}

The elevated zero maze (EZM) is an effective tool to measure anxiety in rodents [527]. The apparatus consists of a circular runway (diameter $46 \mathrm{~cm}$, width $5.5 \mathrm{~cm}$ ) made from black plastic material that was transparent for infrared light and elevated $20 \mathrm{~cm}$ above the floor. The arena was divided into 4 quadrants: two opposing open quadrants with a low border (height $3 \mathrm{~mm}$ ) to prevent the mouse from stepping down, and 2 opposing closed quadrants with sidewalls (height $11 \mathrm{~cm}$ ). Each mouse was placed individually into one of the open arms and was allowed to explore the arena for 5 minutes under low-light conditions. After each session the maze was thoroughly cleaned using ethanol and water and dried. The movements of each mouse were scored automatically using an infrared camera and Ethovision Pro (Noldus Information Technology, Wageningen, The Netherlands). The parameter for anxious behavior was the percentage of time spent in the open quadrants of the maze, corrected for freezing behavior by subtracting the seconds it takes to move into a closed arm from the total time spent in the arena. In addition, total distance moved was taken as a measure of locomotor behavior.

\subsubsection{Forced swim test}

The forced swim test (FST) is the most commonly used behavioral task to study depressivelike behavior in rodents [529]. Under low light conditions, each mouse was individually placed into a Perspex cylinder (diameter $17 \mathrm{~cm}$, height $40 \mathrm{~cm}$ ) filled with $30^{\circ} \mathrm{C}$ water to a height of $15 \mathrm{~cm}$ for 6 minutes. Each session was scored automatically using a camera and Ethovision Pro (Noldus Information Technologies, Wageningen,The Netherlands). Total distance swum by the mouse was taken as a measure of depressive-like behavior [542].

\subsection{Blood sampling and corticosterone radioimmunoassay}

In order to determine the plasma levels of corticosterone in these mice, blood samples were taken as follows. First, the mouse was taken out of its home cage, and a 'basal' blood sample was drawn from the vena saphena using heparinized blood collection tubes (Microvette ${ }^{\circledR}$ CB300, Sarstedt, Germany). Subsequently, the mouse was restrained in a 
glass cylinder to induce a stress response. After 20 minutes of restraint stress another 'stress' blood sample was drawn from the vena saphena. All blood samples were kept on ice and subsequently centrifuged at $3000 \mathrm{~g}$ for 10 minutes at $4^{\circ} \mathrm{C}$ after which plasma was isolated and frozen down to $-80^{\circ} \mathrm{C}$ for subsequent determination (in duplicate). For the determination of plasma corticosterone levels, the ImmuChem ${ }^{\mathrm{TM}}$ Double Antibody Corticosterone ${ }^{125}$ I RadiolmmunoAssay Kit for rodents (MP Biomedicals, Orangeburg, NY, USA) was used. The assay was performed according to the manufacturer's instructions. Briefly, for each sample, $5 \mu \mathrm{l}$ serum was diluted in steroid diluents (1:100 for basal and recovery samples and 1:200 for stress samples). Per $50 \mu \mathrm{l}$ of diluted sample, $50 \mu \mathrm{l}$ of corticosterone ${ }^{-125} \mathrm{I}$ and $100 \mu \mathrm{l}$ anti-corticosterone was added, and samples were incubated at room temperature (RT) for $2 \mathrm{~h}$. Afterward, $250 \mu \mathrm{l}$ precipitant solution was added and tubes were centrifuged at $2300 \mathrm{rpm}$ for $15 \mathrm{~min}$. Supernatant was aspirated and the precipitate was counted in a Wizard Gamma Counter 2470 (Perkin Elmer, Waltham, MA, USA).

\subsection{Tissue preparation}

Mice were decapitated and their brains were carefully removed. All brains were halved down the mid-sagittal line. The left hemisphere was immersion fixated for 2 days using $4 \%$ buffered paraformaldehyde and cryo-protected by immersion in $30 \%$ sucrose in $0.1 \mathrm{M}$ phosphate buffer at $4^{\circ} \mathrm{C}$ overnight. Subsequently, the tissue was quickly frozen using $\mathrm{CO}_{2}$ and stored at $-80^{\circ} \mathrm{C}$ until further processing. The left hemisphere of 5-7 animals per group was cut into entire free floating series of $30 \mu \mathrm{m}$ thick coronal sections using a cryostat. Every tenth section was systematically assigned to one series. Accordingly, every series is representative for the whole selected brain area. After cutting, the material was stored at $80^{\circ} \mathrm{C}$ until further processing.

The right hemisphere was microdissected, isolating the hippocampus, frontal cortex and the rest of the brain. Samples were snap frozen in liquid nitrogen after which they were stored at $-80^{\circ} \mathrm{C}$ until further analysis. Hippocampal tissue was homogenized using a Bead Beater (Biospec products, OK, USA) for 2 times 30 seconds in $2 \mathrm{ml}$ ice-cold lysis buffer (137 $\mathrm{mM} \mathrm{NaCl}, 20 \mathrm{mM}$ Tris-HCl ( $\mathrm{pH} 8.0$ ), 1\% Igepal, 10\% glycerol and 1 complete protease inhibitor tablet; Roche, The Netherlands) and stored at $-80^{\circ} \mathrm{C}$ until further processing as described in section 2.8 .

\subsection{Immunohistochemistry}

All rinsing in between incubation and blocking steps was performed in Tris buffered saline with $0.2 \%$ Triton X-100 (TBS-T), TBS, TBS-T, for 3 times 10 minutes, unless otherwise specified.

\subsubsection{A immunohistochemistry}

To determine hippocampal plaque load, a triple-fluorescent immunohistochemical staining was performed, using the $6 \mathrm{E} 10$ antibody to stain all $A \beta$, Thioflavine $S$ to stain the highly 
fibrillar dense-core plaques and Hoechst labeling as a counterstain. Incubation with the primary anti-A $1-17$ antibody (6E10 clone, 1:1200, Sigma, Zwijndrecht, the Netherlands) occurred overnight in TBS-T at $4^{\circ} \mathrm{C}$. Subsequent incubation with Alexa 594 (donkey antimouse, 1:200, Breda, The Netherlands) was performed for $2 \mathrm{~h}$ in TBS-T at RT, followed by 10 min incubation with Thioflavine $S(0.0075 \%)$ in TBS-T at RT. Next, the sections were rinsed for $5 \mathrm{~min}$ in $70 \%$ ethanol and then $10 \mathrm{~min}$ in TBS, before incubating them for $30 \mathrm{~min}$ with Hoechst (1:500, Sigma, Zwijndrecht, the Netherlands) in TBS at RT, after which the sections were mounted and coverslipped in $80 \%$ glycerol in TBS.

\subsection{2. $p C R E B$ immunohistochemistry}

Antigen retrieval was performed by placing the sections overnight in TBS and incubating them for $20 \mathrm{~min}$ in $10 \mathrm{mM}$ citrate acid and a $95^{\circ} \mathrm{C}$ water bath. Afterward, the sections were left to cool down for $20 \mathrm{~min}$, rinsed (3x $10 \mathrm{~min}$ TBS) and blocked for endogenous peroxidases $1 \% \mathrm{H}_{2} \mathrm{O}_{2}$ for 60 minutes. To block aspecific binding, sections were incubated with $5 \%$ normal donkey serum in TBS-T for 30 minutes and subsequently incubated in the primary antibody (anti-pCREB, 1:200, Cell Signaling, Danvers, MA, USA) in 0.5\% normal donkey serum in TBS-T on a constant shaker for 3 nights at $4^{\circ} \mathrm{C}$. Incubation with biotinylated donkey anti-rabbit (1:400; Bio-connect BV, Huissen, the Netherlands) was performed for 90 minutes, after which the signal was enhanced with avedin-biotinperoxidase complex (1:400, Vector Laboratories, Burlingame, CA, USA) at RT for 90 minutes. Next, the sections were washed 3 times for 10 minutes in Tris- $\mathrm{HCl}$ and incubated in 3,3'-diaminobenzidine (DAB, Sigma, Uithoorn, the Netherlands) diluted 1:1 in Tris- $\mathrm{HCl}$ and also containing $0.3 \% \mathrm{H}_{2} \mathrm{O}_{2}$ and $8 \%$ nickel-chloride for 10 minutes at RT. Subsequently, the sections mounted on gelatin-coated glasses, dehydrated using Ultraclear and coverslipped with Pertex (HistolabProducts AB, Göteborg, Sweden).

\subsubsection{Synaptophysin immunohistochemistry}

The sections were mounted on glass the day before staining and dried overnight, after which they were post-fixated using Somogyi's fixation solution (4\% paraformaldehyde, $15 \%$ picric acid, $0.05 \%$ glutaraldehyde in $0.1 \mathrm{M}$ phosphate buffer) for 20 minutes. After incubation with $0.3 \% \mathrm{H}_{2} \mathrm{O}_{2}$ for 30 minutes at RT to block endogenous peroxidases, section with incubated with $5 \%$ normal donkey serum in TBS-T for 30 minutes at RT. Incubation with the primary mouse anti-synaptophysin antibody (1:2000, Millipore, Amsterdam, the Netherlands) in TBS$\mathrm{T}$ with $0.5 \%$ normal donkey serum at RT occurred over 3 nights in a humid box. Two hour incubation with biotinylated donkey anti-mouse antibody (1:200, Bio-connect BV, Huissen, the Netherlands) in TBS-T with $0.5 \%$ normal donkey serum was performed followed by 90 minutes incubation with the avedin-biotin-peroxidase complex (1: 400, Vector laboratories) at $\mathrm{RT}$, rinsed for 3 times $10 \mathrm{~min}$ in $\mathrm{Tris}-\mathrm{HCl}(0.05 \mathrm{M}, \mathrm{pH} 7.6)$ and incubated for 10 minutes with DAB-Tris- $\mathrm{HCl}$ and diluted $1: 1$ in Tris- $\mathrm{HCl}$ and $0.3 \% \mathrm{H}_{2} \mathrm{O}_{2}$. Sections were dried overnight, dehydrated and coverslipped with Pertex. 


\subsection{Analysis of immunoreactivity}

\subsubsection{Plaque load}

All plaque load measurements were performed on a stereology workstation consisting of a modified BX51WI bright field microscope (Olympus, Tokyo, Japan), Olympus UPlanApo objectives (Olympus, Tokyo, Japan), three-axis high-accuracy computer-controled stepping motor specimen stage for automatic sampling (4x4 Grid Encoded Stage; Ludl Electronics, Hawthorne, NY, USA), linear z-axis position encoder (Ludl Electronic Products Ltd, Hawthorne, NY, USA), MBF-CX9000 CCD color camera (1.200x1.800 pixels; CX9000; MBF Bioscience, Williston, VT, USA) and controlling software (MBF Bioscience, Williston, VT, USA). The plaque load in the hippocampus was analyzed using all sections between Bregma $-1.06 \mathrm{~mm}$ to $-3.88 \mathrm{~mm}$. Per animal, an average of 9 sections was analyzed, depending on the individual rostro-caudal extension of the hippocampus. Delineations of the total hippocampus were made using a $10 x$ objective (numerical aperture $(N A)=0.40$ ). Within the delineation, the plaque area was measured with the Area Fraction Fractionator probe of the Stereo Investigator software (MBF Bioscience, Williston, VT, USA) using a 60x objective (oil, $N A=1.35$ ). The plaque load was determined by dividing the total plaque area by the total hippocampal area of the same section.

\subsection{2. $p C R E B$}

For each animal 5 sections were analyzed, 2 in the dorsal hippocampus (Bregma -1.34 to $2.30 \mathrm{~mm}$ ), 1 in the intermediate part of the hippocampus (Bregma -2.30 to -2.92) and 2 in the ventral hippocampus (Bregma -2.92 to $-3.64 \mathrm{~mm}$ ), as adapted from [536]. In each section the DG, CA3 and CA1 were analyzed by randomly selecting sites for analysis. In the DG 5 sites were selected, 2 in the upper blade, 2 in the lower blade and one in the tip; while in the other two subregions 3 sites were randomly selected (see Figure 7A for a representation of where the photomicrographs are taken). In brief, a photomicrograph was taken at every selected site with with a digital camera (F-view; Olympus, Tokyo, Japan) attached to an Olympus AX-70 microscope (40x oil objective; Olympus UplanApo, NA=1.00). This resulted in approximately 55 photos per animal. In these photomicrographs pCREB immunoreactivity (IR) was analyzed by measuring the mean gray value of pCREB IR (reflecting the intensity) and the surface area of $\mathrm{pCREB} I R$ within the region of interest by using ImageJ software (version 1.42q, Wayne Rasband, National Institues of Health, Bethesda, Maryland, USA). In brief, within each photomicrograph the cell layer was delineated, thereby excluding hippocampal neuropil from the analysis. Background corrections were performed by setting the same minimum threshold value for every photomicrograph [535]. The mean gray value was defined as the sum of gray values of all pixels in the delineated area divided by the number of all pixels in this region. Surface area was defined as the percentage of the delineated area with a gray value above the background threshold. 


\subsubsection{Synaptophysin-immunopositive presynaptic boutons}

The sections stained for synaptophysin were analyzed ranging from Bregma $-1.34 \mathrm{~mm}$ to $3.64 \mathrm{~mm}$. Per animal, an average of 7 sections was analyzed, depending on the individual rostro-caudal extension of the hippocampus. Three subregions within the hippocampus were assessed, i.e. the stratum radiatum of CA1, the stratum lucidum of CA3 and the stratum moleculare of the dentate gyrus. Within these subregions, three sites were randomly selected for analysis (see Figure 8A for a representation of where the photomicrographs are taken). The density of synaptophysin immunoreactive presynaptic boutons (SIPBs) was estimated as previously described (Rutten et al. 2005 AmJPath). In brief, a photomicrograph was taken at every selected site with the same set up as described in section 2.7.2. and a $100 x$ oil objective (Olympus UplanApo, NA=1.35), resulting in approximately 63 photos per animal. In these photomicrographs SIPBs were detected by image analysis using the AnalySIS-pro software (Soft Imaging System, Munster, Germany). First, the area of interest was defined in every image. Next, shading error correction was performed to correct for irregularities in illumination of the microscopic fields and a differential contrast enhancement filter (DCE) was applied to selectively enhance weak differences in contrast. Lastly, the detection threshold was tested and kept at the same level for all samples. All measurements were performed on a single focal plane and cell bodies, blood vessels and artifacts, e.g. A $\beta$ plaques were excluded from the analysis. Particles smaller than $0.025 \mu^{2}$ and larger than $10 \mu \mathrm{m}^{2}$ were considered noise and not taken into account in the analysis. From these data the mean SIPB density per $\mu \mathrm{m}^{2}$ was calculated per subregion.

\subsection{Brain-derived neurotrophic factor ELISA}

Protein concentrations in hippocampal homogenates were determined using the Bio-Rad Lowry Protein Assay (Bio-Rad Laboratories Inc., Hercules, USA). Subsequently, the concentration of BDNF was determined using the BDNF $E_{\max }{ }^{\circledR}$ ImmunoAssay System according to manufacturer's protocol with samples being acid treated (Promega, Madison, WI, USA) as described in [554].

\subsection{Statistical analysis}

All data were expressed as mean \pm standard error of the mean (S.E.M.). Repeated Measures ANOVA was performed on the weight monitoring data, the OLT and the corticosterone secretion (LN-transformed), to assess the main and interactive effects of genotype and treatment progression over time, the different intervals or the different conditions (basal, stress), respectively. Two-way ANOVA was performed for comparison between groups accounting for the main and interactive effects of genotype (WT, APPswe/PS1 $\triangle E 9$ ) and treatment (veh, BAY60-7550) on the Y-maze, EZM, FST, plaque load, pCREB IR, BDNF concentrations and SIPB density. When an interaction effect or main effect for treatment was observed, a student's t-test was performed to assess whether treatment effects were specific for, or more pronounced in a certain genotype. To assess the 
level of memory function compared to chance level performance, one-sample t-tests were performed for the $\mathrm{d} 2$ and alternations measure in the Y-maze and OLT experiment, respectively, with values set at no memory performance, i.e. 50 and 0 , respectively. For all immunohistochemical data, a subdivision between the dorsal (Bregma -1.06 to $-2.30 \mathrm{~mm}$ ) and the ventral hippocampus (Bregma -2.92 to $-3.88 \mathrm{~mm}$ ) was made (adapted from [533]). Statistical significance was set at an a level of 0.05 and all tests were two-tailed. All statistical analyses were conducted using the Statistical Package for the Social Sciences (SPSS 15.0, SPSS Inc., Chicago, IL, USA). All graphs were constructed in GraphPad Prism (version 4, GraphPad Software, San Diego, CA, USA).

\section{Results}

\subsection{Weight monitoring}

Repeated Measures ANOVA revealed a significant time $(F(3.23,167.75)=16.40, p<0.001)$ and time* genotype effect $(F(3.23,167.75)=5.99, p<0.001)$, with APPswe/PS1 $\Delta E 9$ mice being significantly heavier, but no effects for time treatment $(F(3.23,167.75)=0.29$, n.s. $)$, or time* ${ }^{*}$ enotype*treatment $(F(3.23,167.75)=0.94$, n.s. $)$. In addition, neither chronic injections nor behavioral testing per se induced significant weight changes, as can be seen in Figure 2 .

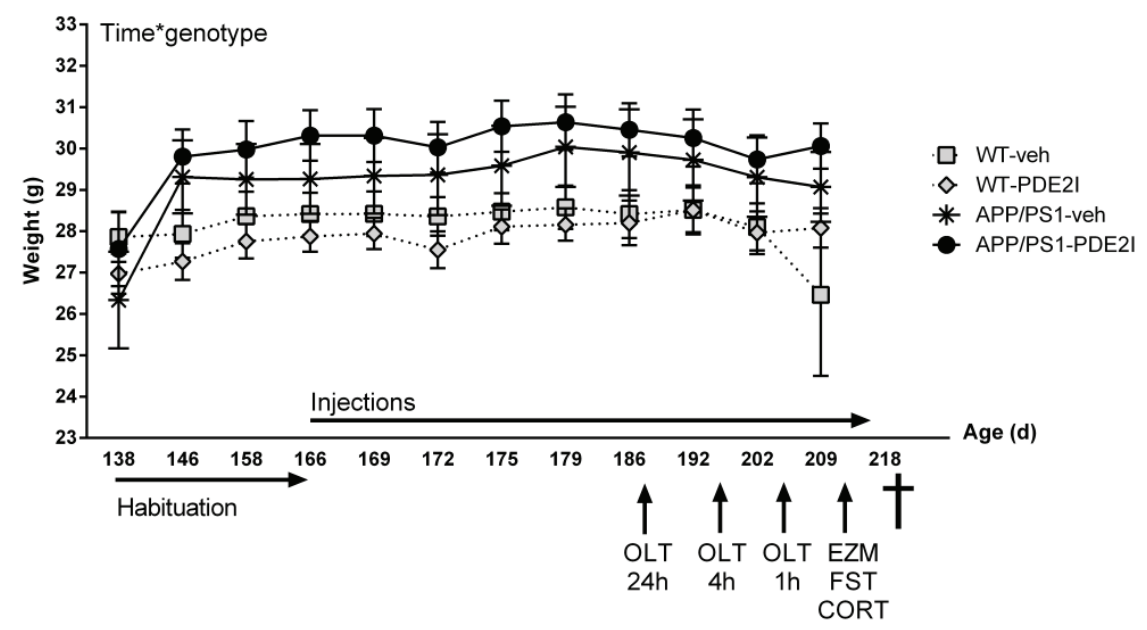

Figure 2: Weight alterations over the course of the experiment, with the age of the mice expressed in days (d). APPswe/PS1 $\triangle \mathrm{E} 9$ mice were significantly heavier than WT mice over the course of the experiment (time* genotype, $\mathrm{p}<0.001$ ). In addition, it can be seen that the chronic injections or behavioral experiments did not induce weight alterations. CORT: blood withdrawal for corticosterone measurements; EZM: elevated zero maze; FST: forced swim test; OLT: object location task. 


\subsection{Behavioral data}

\subsubsection{Object location task}

The results of the OLT are summarized in Table 1 and Figure 3. A Repeated Measures ANOVA on e1 and e2 indicated that genotype had an overall effect on exploration time (e1: $(F(1,52)=7.74, \quad p<0.01)$; e2: $(F(1,52)=3.56,0.1<p<0.05))$ with APPswe/PS1 $1 E 9$ mice spending more time exploring the objects (see Table 1). Treatment $(e 1:(F(1,52)=0.07$, n.s.); e2: $(F(1,52)=0.08$, n.s. $))$ or genotype*treatment $(e 1:(F(1,52)=0.19$, n.s. $)$; e2: $(F(1$, $52)=1.94$, n.s.)) did not affect exploration times. The repeated measures ANOVA on the relative d2 discrimination index, which corrects for differences in exploratory activity, indicated no effect of interval $(F(2,96)=2.01$, n.s. $)$, interval ${ }^{*}$ genotype $(F(2,96)=0.55$, n.s. $)$, interval ${ }^{*}$ treatment $(F(2,96)=0.20$, n.s. $)$ or interval ${ }^{*}$ genotype treatment $(F(2,96)=2.02$, n.s. $)$. However, the test of between-subject effects did indicate an overall trend toward an effect of genotype $(F(1,48)=2.97, p=0.091)$ on the $d 2$, with APPswe/PS1 $\triangle E 9$ mice having lower $d 2$ scores, while treatment $(F(1,48)=1.80$, n.s. $)$ and genotype treatment $(F(1,48)=0.40$, n.s. $)$ did not. To investigate which groups had functional spatial memory at which interval, a onesample t-test was performed. After a $1 \mathrm{~h}$ and $4 \mathrm{~h}$ interval, all groups were able to significantly identify the new location of the object $(\mathrm{t}(>11)>2.31, \mathrm{p}<0.05)$. However, after a $24 \mathrm{~h}$ interval the APPswe/PS1 $\triangle E$ 9-veh group could not distinguish the new location from the old location $(t(11)=1.83, p=0.09)$, while the other groups could $(t>12)>4.68, p<0.01)$. Interestingly, APPswe/PS1 1 E9 mice treated with BAY60-7550 showed an almost identical D2 score as the WT-veh animals during all intervals (see Figure 3).

\subsubsection{Y-maze}

WT-veh mice showed a statistical trend toward functional working memory $(t(1,14)=2.06$, $\mathrm{p}=0.058$ ), while APPswe/PS1 $\triangle \mathrm{E} 9$-veh mice did not perform significantly better than chance level $(t(1,12)=1.23$, n.s. $)$. Chronic BAY60-7550 treatment led to a performance significantly better than $50 \%$ in both the WT $(t(1,14)=3.60, p<0.01)$ and the APPswe/PS1 $\triangle E 9$ mice $(t(1$, $12)=3.37, p<0.01$ ) (see Figure 4). Two-way ANOVA could not detect effects of genotype $(F(1,55)=0.27$, n.s. $)$, treatment $(F(1,55)=0.30$, n.s. $)$ or genotype*treatment $(F(1,55)=0.32$, n.s.) on percentage of alternation between the groups. A significant genotype effect was found for the number of entries and triads made in the $Y$-maze $((F(1,52)=14.19, p<0.01)$, $(F(1,52)=9.49, p<0.01)$, respectively), with APPswe/PS1 $\triangle E 9$ mice making more of each (see Table 2). No significant effects were found of treatment (entries: $(F(1,55)=0.17$, n.s.), triads: $(F(1,55)=0.01$, n.s. $)$ or genotype treatment (entries: $(F(1,55)=0.71$, n.s. $)$, triads: $(F(1$, $55)=1.46$, n.s.). 


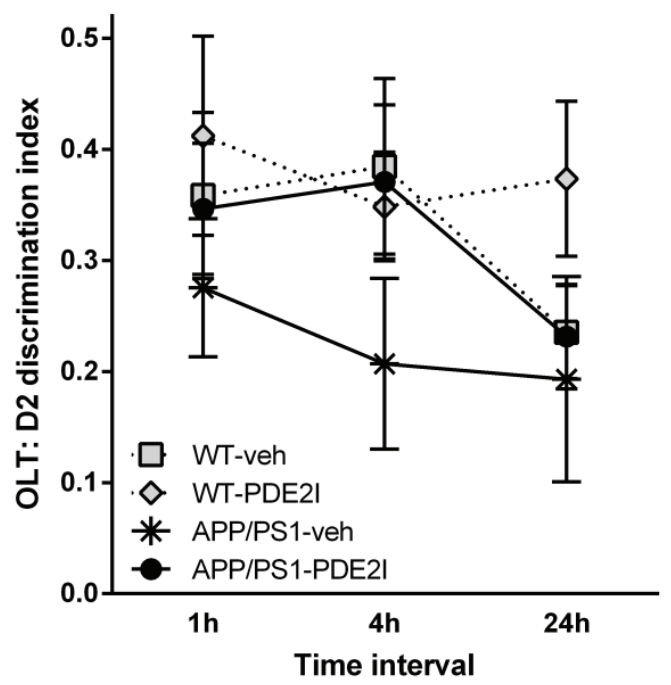

Figure 3: Performance in the object location task (OLT) over 3 intervals (1h, 4h, 24h). Values indicate mean and S.E.M per group. Although all animals were able to significantly distinguish between the novel and the familiar location at the $1 \mathrm{~h}$ and $4 \mathrm{~h}$ interval, i.e. D2 $>0, p<0.05$, it is interesting to note that the APPswe/PS1 $\triangle$ E9-PDE2-I group has almost the same absolute D2 scores as the WT-veh group in all intervals. At the $24 \mathrm{~h}$ interval APPswe/PS1 $\triangle \mathrm{E} 9$-veh group is not significantly able to distinguish between the novel and the familiar location, while the other groups are.

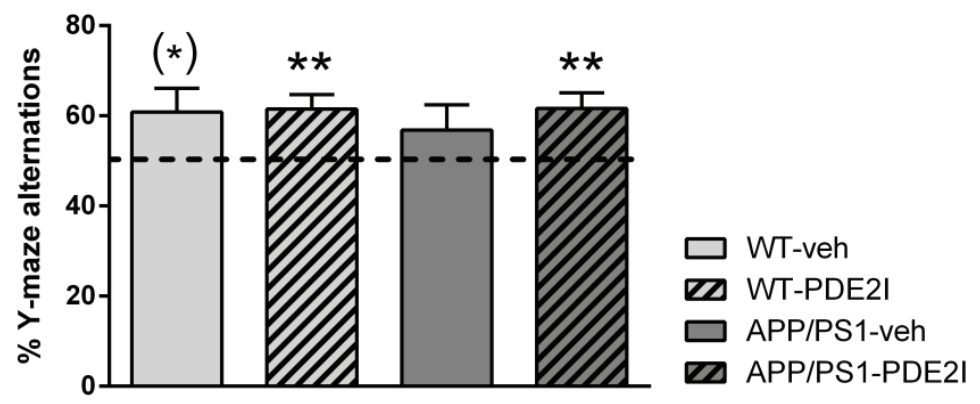

Figure 4: Working memory performance, as measured by the percentage of alternations in the Y-maze. Bars represent mean and S.E.M per group. Performance of each group was compared to $50 \%$, as this is the chance level for choosing the correct arm. Only mice chronically treated with the PDE2-I BAY607550 perform significantly better than chance level $(50 \%)$. WT-veh mice show a trend toward functional working memory, while APPswe/PS1 $\Delta$ E9-veh mice do not. ${ }^{* *}: p<0.01 ;\left(^{*}\right): 0.05<p<0.1$. 


\subsubsection{Elevated zero maze}

No difference could be observed between the groups in the time spent in the open arms of the $E Z M$, indicating no difference in anxious behavior (genotype: $(F(1,55)=0.81$, n.s.), treatment $(F(1,55)=2.36$, n.s. $)$, genotype*treatment $(F(1,55)=1.51$, n.s. $)$. In addition, the total distance moved in the arena was not different among groups (see Table 2) (genotype: $(F(1,55)=1.39$, n.s. $)$, treatment $(F(1,55)=1.47$, n.s. $)$, genotype treatment $(F(1,55)=0.85$, n.s.).

\subsubsection{Forced swim test}

Neither genotype $(F(1,55)=0.61$, n.s. $)$, treatment $(F(1,55)=.24$, n.s. $)$ nor the interaction of genotype*treatment $(F(1,55)=1.04$, n.s. $)$ had an effect on the total distance swum in the FST, indicating no differences in depressive-like behavior (see Table 2).

\subsection{Corticosterone measurement}

Repeated Measures ANOVA demonstrated a significant time effect $(F(1,41)=258.88$, $p<0.001)$, but no effects for time* genotype $(F(1,41)=0.49$, n.s. $)$, time*treatment $(F(1$, $41)=0.12$, n.s. $)$ or time ${ }^{*}$ genotype ${ }^{*}$ treatment $(F(1,41)=2.62$, n.s. $)$ on corticosterone concentrations. However, between-subject analysis indicated an overall genotype effect $(F(1,41)=6.27, \quad p<0.05)$, with APPswe/PS1 $\triangle E 9$ mice having higher corticosterone concentrations. Overall effects for treatment $(F(1,41)=0.01$, n.s. $)$ or genotype*treatment $(F(1,41)=1.07$, n.s.) were not observed. One-way ANOVAs on the individual time points, showed that genotype had a significant effect on both the basal corticosterone secretion $(F(1,53)=4.15, p<0.05)$ and the stress-induced secretion $(F(1,46)=5.25, p<0.05)$. Treatment did not influence corticosterone concentrations, neither in the basal $(F(1,53)=0.30$, n.s.) nor in the stressful condition $(F(1,46)=0.07$, n.s.). The interaction of genotype*treatment did tend to affect basal corticosterone secretion $(F(1,53)=3.13, p=0.08)$, with BAY60-7550 treatment lowering basal corticosterone levels in WT but increasing concentrations in APPswe/PS1 $\triangle \mathrm{E} 9$ mice. Post hoc analysis did not reveal any significant effects of treatment within each genotype. No interaction effect was observed in the stress-induced corticosterone concentrations $(F(1,46)=0.00$, n.s.) (see Figure 5). 


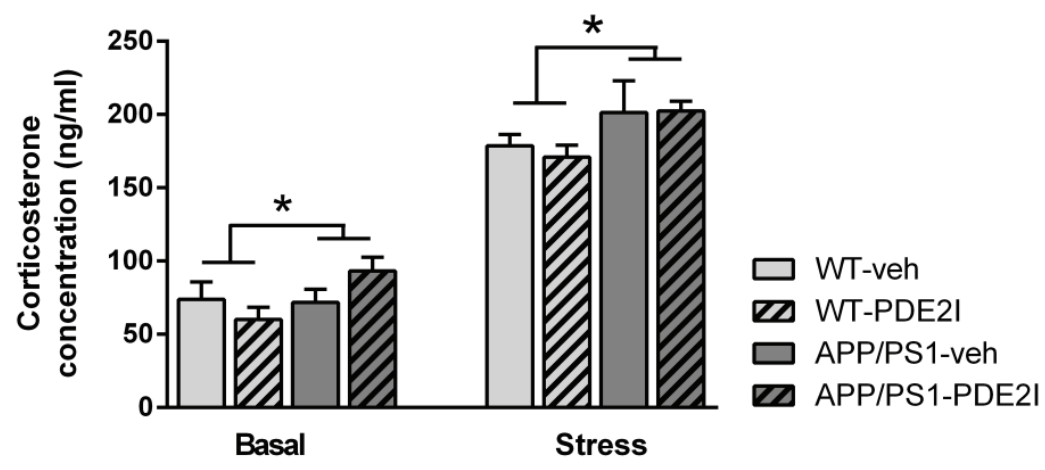

Figure 5: Corticosterone concentrations in blood plasma under basal and stressful conditions; bars indicate mean and S.E.M. APP/PS1 mice had significantly higher corticosterone concentrations at both timepoints. *: APPswe/PS1 $\triangle \mathrm{E} 9$ vs WT, $p<0.05$.

\subsection{Plaque load}

Chronic BAY60-7550 treatment did not affect plaque load in the dorsal $(F(1,9)=0.70$, n.s. $)$, ventral $(F(1,9)=1.31$, n.s. $)$ or the total hippocampus $(F(1,9)=0.88$, n.s. $)$ in APPswe/PS1 $\triangle E 9$ mice (see Figure 6).

\section{5. $p C R E B$ IR}

Neither genotype, treatment nor genotype treatment affected pCREB IR intensity in the DG (genotype $(F(1,22)=0.09$, n.s. $)$, treatment $(F(1,22)=0.00$, n.s. $)$, genotype treatment $(F(1$, $22)=0.01$, n.s.)), CA3 (genotype $(F(1,22)=0.01$, n.s.), treatment $(F(1,22)=0.02$, n.s.), genotype treatment $(F(1,22)=0.02$, n.s.)) or $C A 1$ subregion of the hippocampus (genotype $(F(1,22)=0.10$, n.s. $)$, treatment $(F(1,22)=0.23$, n.s. $)$, genotype ${ }^{*}$ treatment $(F(1,22)=0.27$, n.s.)) (see Figure 7). 

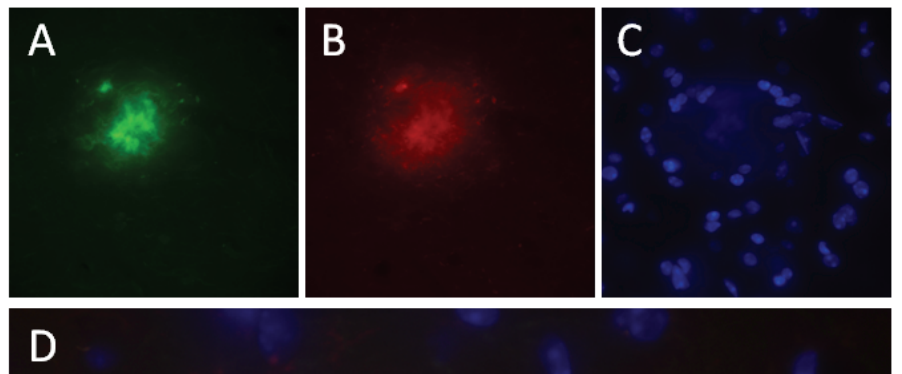

E

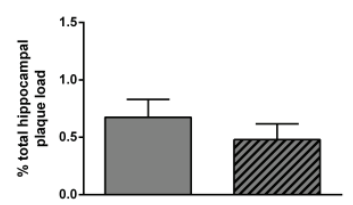

$\mathbf{F}$

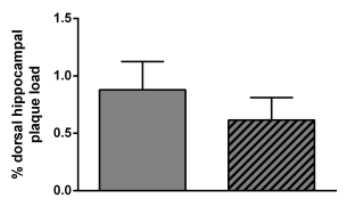

G

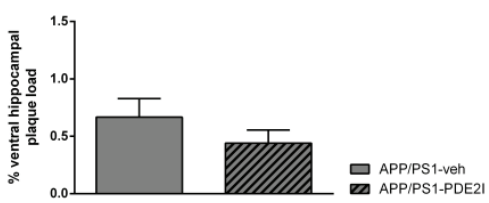

Figure 6: Hippocampal plaque load. A-D: representative images of a plaque in the hippocampus of an APPswe/PS1 $\triangle E 9$ mouse at $60 x$, using Thioflavine $S$ to visualize dense core plaques (A), $6 E 10$ for immunoreactivity against Abeta1-16 (B) and Hoechst as a counterstain (C), which are merged in D. Scale bar represents $20 \mu \mathrm{m}$. E-G: analysis of hippocampal plaque load in the total $(E)$, dorsal $(F)$ and ventral hippocampus $(G)$. Bars indicate mean and S.E.M. Chronic PDE2-I treatment did not affect plaque load in APPswe/PS1 $\triangle E 9$ mice. WT mice do not develop $A \beta$ plaques and were therefore omitted from analysis. 


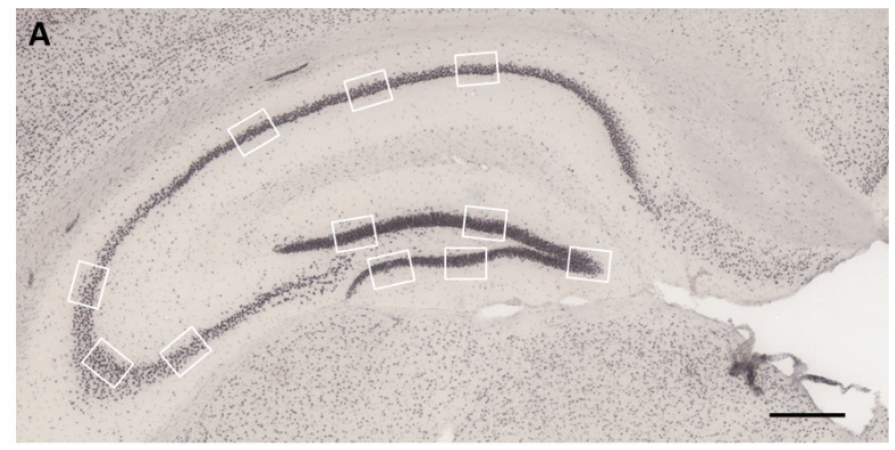

B

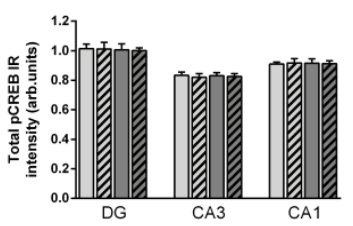

C

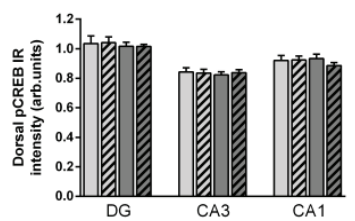

D

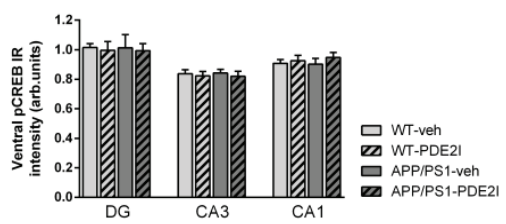

Figure 7: Phosphorylated CREB (pCREB) immunoreactivity (IR). A; Representative image of pCREB IR in the dorsal hippocampus. White rectangles represent sites at which high-magnification photomicrographs were taken for quantitative analysis of pCREB IR (per section, 5 sites for the dentate gyrus, 3 for the CA3, and 3 for the CA1 were analyzed. See Section 2.7.2. for more details). No differences could be found between the groups in pCREB IR in the total $(B)$, dorsal (C) or ventral hippocampus (D). Scale bar represents $500 \mu \mathrm{m}$.

\subsection{SIPB density}

A significant genotype effect was found in total stratum lucidum of the CA3 $(F(1,22)=4.51$, $p<0.05)$. Separate analysis of the dorsal and ventral part, revealed that genotype significantly affected the dorsal stratum lucidum $(F 1,22)=5.43, p<0.05)$, while a trend for a genotype effect was observed for the ventral stratum lucidum $(F(1,22)=3.51, p=0.076)$. Moreover, the ventral stratum moleculare of the DG was also significantly affected by genotype $(F(1,22)=6.03, p<0.05)$. In all these areas APPswe/PS1 $\triangle E 9$ mice had higher SIPB density compared to WT mice (see Figure 8 ). The other regions remained unaffected by genotype (stratum moleculare: total $(F(1,22)=2.42$, n.s.), dorsal $(F(1,22)=0.22$, n.s.); stratum radiatum: total $(F(1,22)=1.23$, n.s. $)$, dorsal $(F(1,22)=1.30$, n.s. $)$, ventral $(F(1$, $22)=0.09$, n.s.)). Treatment tended to affect the dorsal stratum lucidem of the CA3 as well $(F(1,22)=3.35, p=0.083)$, with chronic BAY60-7550 treatment lowering the SIPB density. However, no treatment effects could be observed in the total $(F(1,22)=1.38$, n.s.) or ventral stratum lucidum $(F(1,22)=1.84$, n.s. $)$, the stratum moleculare (total $(F(1,22)=0.18$, n.s.), dorsal $(F(1,22)=1.64$, n.s. $)$, ventral $(F(1,22)=0.21$, n.s. $))$, or the stratum radiatum (total $(F(1$, $22)=0.29$, n.s. $)$, dorsal $(F(1,22)=1.53$, n.s. $)$, ventral $(F(1,22)=0.32$, n.s. $))$. SIPB density also remained unaffected by genotype ${ }^{*}$ treatment in the stratum moleculare (total $(F(1,22)=0.03$, 
n.s.), dorsal $(F(1,22)=0.23$, n.s. $)$, ventral $(F(1,22)=0.19$, n.s. $))$, the stratum lucidum (total $(F(1,22)=0.05$, n.s. $)$, dorsal $(F(1,22)=0.01$, n.s. $)$, ventral $(F(1,22)=0.00$, n.s. $)$ and the stratum radiatum (total $(F(1,22)=0.05$, n.s.), dorsal $(F(1,22)=0.04$, n.s. $)$, ventral $(F(1$, 22) $=0.77$, n.s.)).
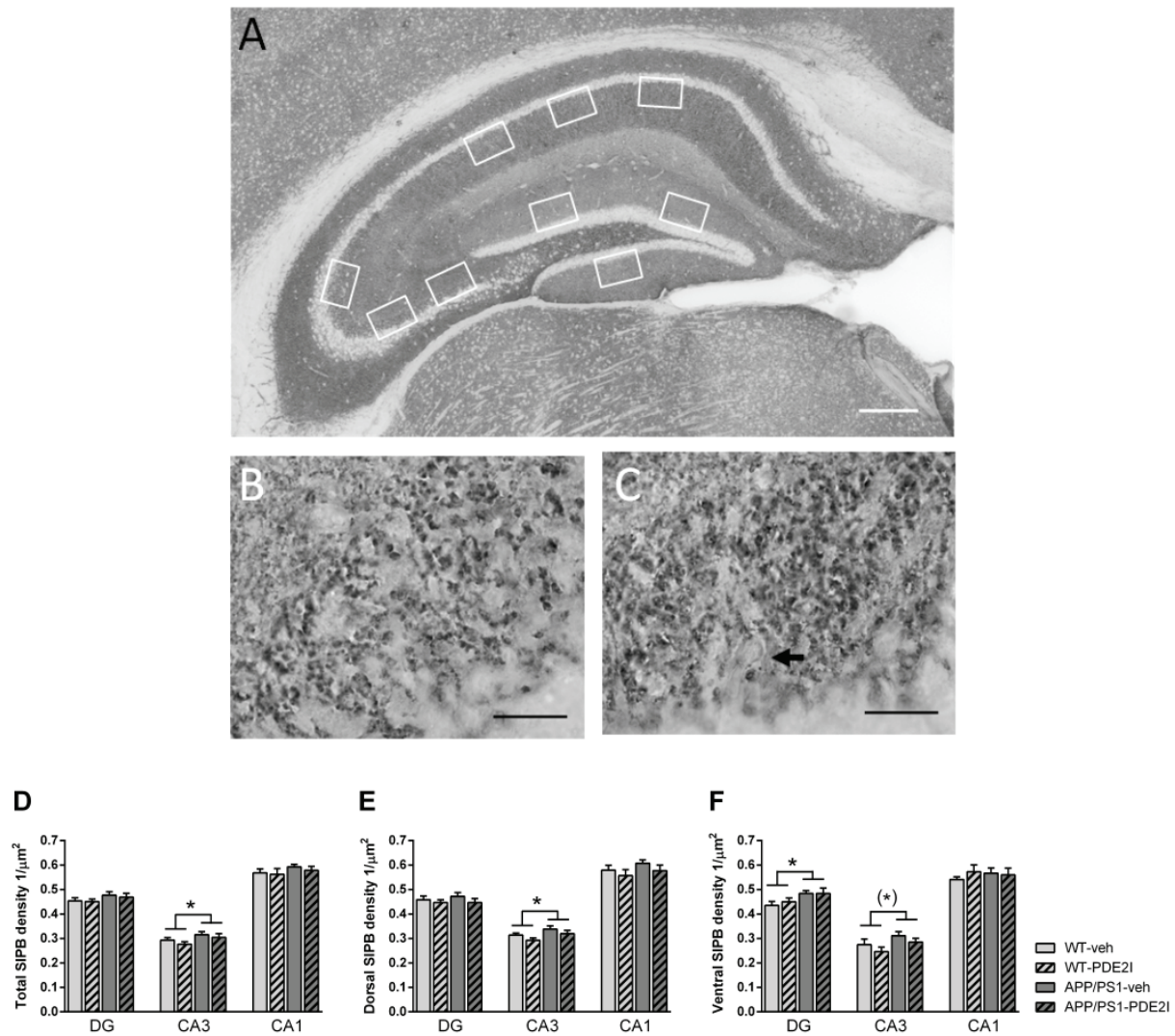

Figure 8: Synaptophysin-immunoreactive presynaptic boutons (SIPBs) in the hippocampus. A-C: representative image of the dorsal hippocampus at 10x (A) and the stratum lucidem of the CA3 subregion at 100x of a WT mouse (B) and an APPswe/PS1 $1 \mathrm{E} 9$ mouse (C). White rectangles in $A$ represent sites at which high-magnification photomicrographs were taken for quantitative analysis of SIPB density (per section, 3 sites for the stratum moleculare, 3 for the stratum lucidem, and 3 for the stratum radiatum were analyzed. See Section 2.7.3. for more details). Black arrow in C points to a blood vessel, which is omitted from analysis. D-F: Analysis of the SIPB density in the stratum moleculare of the DG, the stratum lucidem of the CA3 and the stratum radiatum of the CA1 in the total $(D)$, dorsal $(E)$ and ventral $(F)$ hippocampus revealed that APPswe/PS1 $\triangle E 9$ mice have a significantly higher SIPB density in the total, dorsal and ventral stratum lucidem and the ventral stratum moleculare than the WT mice. Scale bars represent $500 \mu \mathrm{m}$ in $A$ and $10 \mu \mathrm{m}$ in $B+C .{ }^{*}: p<0.05,\left(^{*}\right): 0.05<p<0.1$. 


\subsection{Hippocampal BDNF concentrations}

Hippocampal BDNF protein concentrations were not different among the groups (see Figure 9) (genotype $(F(1,45)=0.06$, n.s. $)$, treatment $(F(1,45)=0.03$, n.s. $)$, genotype treatment $(F(1$, $45)=1.04$, n.s.).

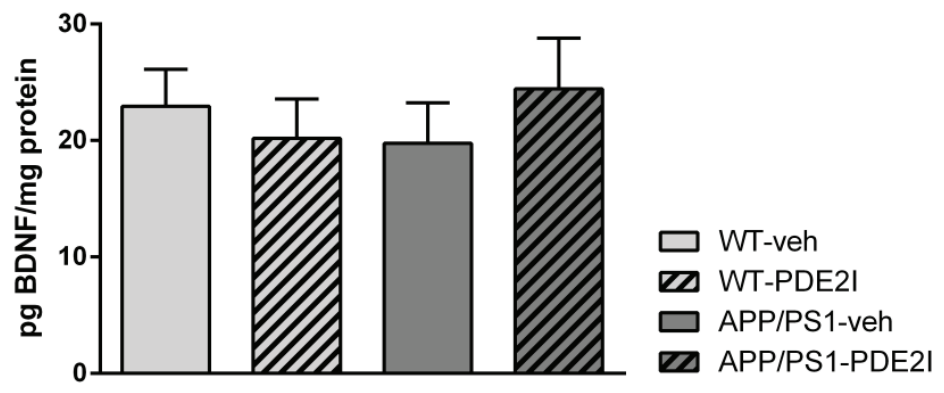

Figure 9: Hippocampal brain-derived neurotrophic factor (BDNF) concentrations. No significant differences were found between the groups.

\section{Discussion}

Our results demonstrate that chronic BAY60-7550 treatment could ameliorate memory function in APPswe/PS1 $1 \mathrm{E} 9$ mice. Remarkably, there were no significant effects on $A \beta$ deposition, CREB phosphorylation, BDNF levels or synaptic density in the hippocampus. Of note, regular weight monitoring revealed that APPswe/PS1 $1 \mathrm{E} 9$ mice were on average heavier than WT mice. Indeed, both weight gain and weigh loss is more often observed in $A D$ patients than control subjects $[618,619]$, and other $A D$ mouse models report similar findings $[620,621]$.

\subsection{Chronic BAY60-7550 treatment improves spatial memory, without affecting anxiety, depressive-like behavior or HPA axis regulation}

When investigating long-term spatial memory, OLT data demonstrated that, overall, APPswe/PS1 $1 \mathrm{E} 9$ animals perform worse than WT mice, particularly at the $24 \mathrm{~h}$ interval of the OLT. Yet, chronic BAY60-7550 treatment ameliorated APPswe/PS1 $\Delta$ E9 performance to a level comparable with the WT-veh animals, i.e. showing intact spatial memory performance. Our findings corroborate the memory-enhancing potential of BAY60-7550 in adult and aged rats and mice $[613,615]$. It should be noted that in our study the memory enhancing effects of BAY60-7550 could not be attributed to acute treatment effects, as the drug was always administered at the end of the day, after behavioral tests were completed. In addition, during the $24 \mathrm{~h}$ interval, animals did not receive an injection on the day of $\mathrm{t} 1$ to exclude any acute drug effect on memory consolidation. 
It is worth mentioning that APPswe/PS1 $\triangle \mathrm{E} 9$ animals explored the objects significantly more than WT animals, as demonstrated in Table 1. This elevated level of activity in APPswe/PS1 $1 \mathrm{E} 9$ animals was also apparent in the Y-maze by the increased number of entries and triads. Exploration in the OLT was defined as active exploration of the object, i.e. sniffing, licking or biting the object, and an increased exploration time could indicate a heightened interest in the object [622]. Conversely, higher exploration times would also enable the animal to remember the object better, as repetition is of crucial importance for memory consolidation [623]. Markedly, despite higher exploration time with the objects APPswe/PS1 $1 \mathrm{E} 9$ mice failed to discriminate the relocated object, indicative of spatial memory impairment. Accordingly, the first hippocampus-related deficits in this mouse model have indeed been reported from 7 months of age [52].

Spatial working memory was assessed by spontaneous alternation in the Y-maze. The data imply that only WT and APPswe/PS1 $\triangle E 9$ mice treated with BAY60-7550 were able to perform significantly above chance level, although these effects were modest. Previous work has demonstrated that acute BAY60-7550 treatment augmented working memory in WT mice in the T-maze [615]. Overall, combining previous work on the efficacy of BAY60-7550 with the results in our memory tests, we can conclude that BAY60-7550 can exert a beneficial effect on memory function.

Although PDE-ls have been proposed as possible anxiolytics and anti-depressants [624-628], the literature remains controversial. Depending on the family specificity of the PDE-I and the chronicity of use, PDE-I treatment can either be anxiolytic or anxiogenic [625, 628-630]. In the present study, we did not find any effects of chronic PDE2-I treatment on anxiety or depressive-like behavior, as measured by the EZM and FST, respectively, neither in WT nor APPswe/PS1 $\triangle \mathrm{E} 9$ mice at about 8 months of age. This indicates that the cognition-enhancing effects of BAY60-7550 are not mediated via alterations in affective processing. Chronic BAY60-7550 treatment also did not affect HPA-axis regulation, despite the widespread presence of PDE2 in the pituitary, hypothalamus and hippocampus $[69,616$, 617]. However, basal and stress-induced corticosterone secretion was upregulated in APPswe/PS1 $1 \mathrm{E} 9$ mice, independent of treatment, compared to WT mice. HPA axis dysfunction has previously been demonstrated in $A D$ patients [107] as well as in other $A D$ mouse models [620, 621, 631]. Together these results indicate that PDE2-I does not globally affect brain function, as neuroendocrine regulation and emotional processing remained unaltered, but is restricted to improvement of cognitive function.

\subsection{Chronic BAY60-7550 treatment does not alter plaque load, pCREB and BDNF levels, or synaptic density}

Our study indicated that chronic BAY60-7550 administration had memory-enhancing effects independent of alterations in $A \beta$ aggregation. Similar findings were also reported for chronic rolipram and sildenafil administration in different $A D$ mouse models, as neither of them altered cortical $A \beta$ levels or plaque load $[65,66,632]$. Yet, prophylactic sildenafil treatment 
of APP/PS1M146L mice was able to decrease cortical plaque load [67]. More recently, it was shown that the NO/cGMP pathway is capable of altering BACE1 and APP activity and expression, thereby influencing $A \beta$ production $[633,634]$, and providing a protective mechanism which could explain the data presented by Puzzo et al. [67].

Chronic PDE4I as well as PDE5I treatment has shown to increase CREB phosphorylation and induce higher BDNF protein levels in the hippocampus of different $A D$ mouse models $[628,632,635,636]$. To our knowledge, this is the first report to assess the effects of chronic PDE2 inhibition on PCREB and BDNF levels in the hippocampus. Although $A \beta$ peptides have shown to negatively affect CREB phosphorylation [610,637-640], and the APPswe/PS1 $1 \mathrm{E} 9$ mouse model does present abundant $A \beta$ production and deposition at 8 months of age $[50,590]$, no significant differences in pCREB or BDNF levels between APPswe/PS1 $1 \mathrm{E} 9$ and WT mice could be observed. Of note, although others do report lower pCREB and BDNF levels in this mouse model from 9 months of age [639, 641], equal and even higher levels of BDNF have also been reported for this mouse model [642-644]. It can also be noted that the reports demonstrating efficacy of PDE-I administration in restoring PCREB and BDNF levels, all used AD models which showed an initial deficit in these variables [66, 610, 611, 632]. Chronic treatment of PDE-Is in WT mouse models often does not further increase pCREB or BDNF levels [66, 67, 632]. Along similar lines, it could be speculated that as our mouse model does not show an initial deficit in the pCREB/BDNF pathway, chronic PDE2-I cannot further augment this transcription pathway.

Analysis of SIPB density in the stratum moleculare of the DG, stratum lucidum of the CA3 and stratum radiatum of the CA1 revealed only a tendency for BAY60-7550 to decrease SIPB density in the ventral stratum lucidum, whereas SIPB density in the other regions remained unchanged. It was hypothesized that chronic BAY60-7550 treatment would result in structural synaptic augmentation, as it was previously demonstrated that stimulation of the cAMP pathway could increase the number of active presynaptic boutons [645]. Additionally, 24-hour rolipram treatment was able to reverse $A \beta$ 's synaptotoxic effects in hippocampal slices as well as after in vivo administration in APPswe/PS1M146L mice for 3 weeks [646]. Interestingly, it was shown in the same study both ex vivo and in vivo, that high levels of $A \beta$ led to an increase in spine area in the hippocampus. Our results corroborate these findings by Smith et al. [646] as our 8-month-old APPswe/PS1 1 E9 mice demonstrate a higher presynaptic density in the total stratum lucidum of the CA3 subregion and in the ventral stratum moleculare of the DG. Increases in post-synaptic density in the stratum radiatum of the CA1 subregion has also been reported by West and colleagues [647] in 12-month-old APPswe/PS1 $1 \mathrm{E} 9$ mice. In early Braak stage AD patients, an initial rise in cortical synaptic markers has also been observed, followed by significant decreases in later stages [648]. This phenomenon has been attributed to compensatory mechanisms, such as synaptic sprouting or enlargement of the existing synaptic terminals [649, 650], possibly via the synaptotrophic properties of APP and PS1 [647, 651]. Similar to West et al. [647], our data suggest an increase in synaptic density in the CA3 region at a younger age. Given the trisynaptic circuit of the hippocampus, it could be speculated that the increase in synaptic 
density in the CA3 region precedes the synaptic density increase in the CA1 subregion in these mice. However, detailed investigation is required to further elucidate the age-related effects of APP and PS1 overexpression on alterations in synaptic density in different hippocampal subregions of this mouse model.

\subsection{Considerations}

Overall, chronic BAY60-7550 administration in APPswe/PS1 $\triangle E 9$ mice yielded interesting and complex findings. It appeared that chronic BAY60-7550 treatment induced cognitionenhancing effects in APPswe/PS1 $\triangle E 9$ mice without inducing affective or neuroendocrinological effects. Remarkably, no structural changes were observed in downstream targets, such as pCREB and BDNF, or at a morphological level, i.e. alterations in presynaptic density or plaque load. Other PDE-Is, known to target the same signaling pathways as BAY60-7550, have been proven successful in enhancing the expression of pCREB and BDNF, diminishing A production and improving LTP $[66,67]$, which raises the question: what could underlie these differences?

Research has indicated that the cyclic nucleotides cAMP and CGMP and their appropriate PDEs are confined to distinct cellular compartments where they interact with numerous other signaling molecules. The localization of specific PDEs within these compartments is of crucial importance in regulating cellular function [652]. Accordingly, it could be considered that cellular localization of PDE2, PDE4 and PDE5 to particular compartments is crucial for their ability to influence $\mathrm{PCREB}$ activation, $A \beta$ production and/or behavior, although more research is needed to elucidate differences between these PDE subtypes.

An alternative explanation for the observed differences in pCREB activation and BDNF signaling could be the age at which the treatment commenced, as well as the AD mouse model used. Gong et al. [66] started their 3 week rolipram treatment in APPswe/PS1M146L mice at a pre-plaque age. In the current study, APPswe/PS1 $\triangle \mathrm{E} 9$ mice received their first BAY60-7550 injection at 6 months of age at which plaques had already started to develop in the hippocampus, even though plaque burden was still very low and hippocampal function was not yet comprised at this age $[50,283]$. As oligomeric and fibrillar $A \beta$ have a differential effect on CREB phosphorylation [653], it should be considered that the efficacy of PDE-I treatment to induce persistent changes in CREB phosphorylation, is dependent on which type of $A \beta$ species predominates as a consequence of age or the mouse model used. The current study demonstrates that starting BAY60-7550 treatment after plaque pathology has commenced, can still induce cognition-enhancing effects. These are hopeful findings, as treatment of $A D$ patients currently only commences after cognitive deficits are evident and neuropathology is often widely prevalent.

In addition, one should consider the timing of last injection and sacrifice. Our last injection was given 3 days before sacrifice and it could be suggested that our PCREB and BDNF data reflect some type of washout or withdrawal effect. The intracellular signaling 
system has received a boost in CAMP and CGMP on a daily basis for almost 8 weeks consecutively. We chose to stop BAY60-7550 treatment 3 days before sacrifice, to ensure ample washout time and to be able to assess whether chronic BAY60-7550 treatment would induce structural changes in pCREB. However, the sudden stop in PDE-I treatment could have resulted in a system reset, thereby temporarily masking any possible beneficial effects of PDE-I treatment on pCREB levels.

The following considerations should be taken into account as well. PDE2 is expressed highly in brain regions involved in learning and memory, and BAY60-7550 has often been shown to enhance cognitive performance [615, 654-656], indicating BAY60-7550 has central effects. However, our chronic BAY60-7550 treatment was given at a relatively low dose (0.3 $\mathrm{mg} / \mathrm{kg}$ ) and perhaps a higher dose might have been more effective on biochemical and morphological levels [615]. In addition, PDEs are also well-known for their vasodilatory effects, and it cannot be ruled out that an enhanced cerebral blood flow and nutrient transport to the brain underlies the cognition-enhancing effects [657].

\subsection{Concluding remarks}

In conclusion, the present data demonstrates that chronic treatment with the PDE2-I BAY607550 can improve cognitive function in an $A D$ mouse model, even after onset of neuropathology, yet without inducing affective or neuroendocrinological alterations. Surprisingly, PDE2-I treatment did not alter hippocampal $A \beta, p C R E B$ or BDNF levels, although an increase in synaptic density in the stratum lucidum was reported for APPswe/PS1 1 E9 mice compared to WT mice. In conclusion, PDEs in general, and PDE2 specifically, could be considered as promising therapeutic targets for cognition enhancement in $A D$. Further research is needed to elucidate which mechanisms may underlie or contribute to the beneficial behavioral effects of chronic PDE2 inhibition in this mouse model of AD.

\section{Acknowledgements}

This study has been partly funded by the Internationale Stichting Alzheimer Onderzoek (ISAO), grant number 07553 to Dr. Kris Rutten. 


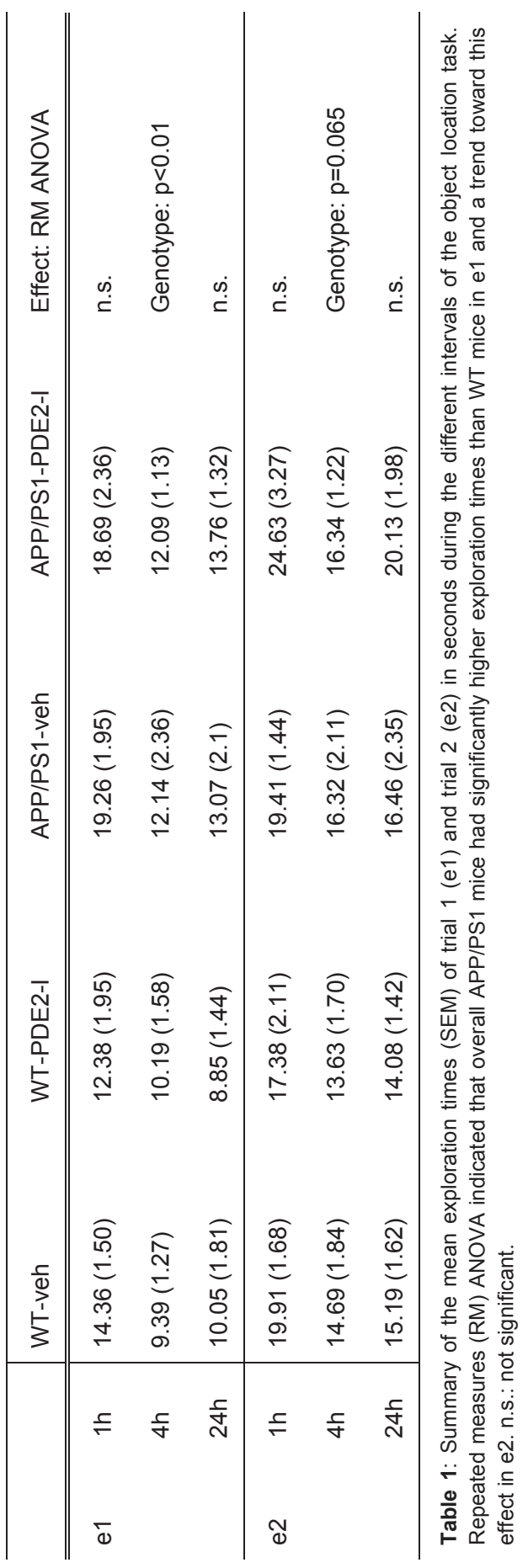




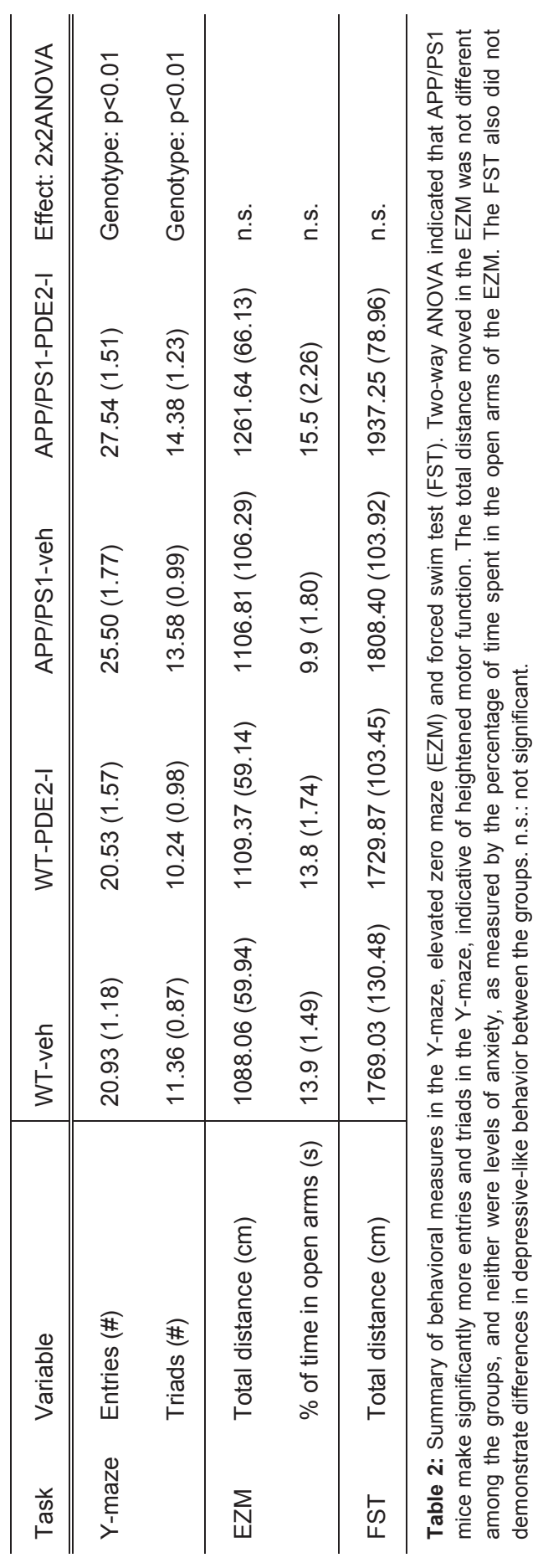




\section{Chapter 7}

Improvement of spatial memory function in APPswe/PS1 $\triangle \mathrm{E} 9$ mice after chronic inhibition of phosphodiesterase type 4D

Annerieke SR Sierksma, Daniel LA van den Hove, Tim Vanmierlo, Franziska Pfau, Marjan Philippens, Olga Bruno, Ernesto Fedele, Roberta Ricciarelli, Harry WM Steinbusch, Jos Prickaerts 


\section{Abstract}

Phosphodiesterase type 4 inhibitors (PDE4-Is) have received increasing attention as cognition-enhancers and could be considered as putative treatments for Alzheimer's disease (AD). By preventing the breakdown of cyclic adenosine monophosphate (cAMP), PDE4-Is can enhance intracellular signal transduction and increase the phosphorylation of cAMP response element-binding protein (CREB) and transcription of proteins related to synaptic plasticity and memory formation. Unfortunately, clinical development of PDE4-Is has been seriously hampered by emetic side effects. The newly developed isoform-specific PDE4D-I, GEBR-7b, has shown to have beneficial effects on memory at non-emetic doses. The aim of the current study was to investigate the cognition-enhancing effects of GEBR-7b in a mouse model of $\mathrm{AD}$. To this extent, 5-month-old APPswe/PS1 $\triangle \mathrm{E} 9$ mice received daily subcutaneous (s.c.) injections with GEBR-7b, vehicle or no injections (to control for injection stress) for a period of 3 weeks, and were tested on affective and cognitive behavior at 7 months of age. We demonstrated a cognition-enhancing potential in APPswe/PS1 $1 \Delta 9$ mice as their spatial memory function at 7 months of age in the object location test was significantly improved by prior GEBR-7b treatment. APPswe/PS1 1 E9 mice displayed lower levels of CREB phosphorylation in the hippocampus, which was not reversed by GEBR-7b treatment. Hippocampal brain-derived neurotrophic factor (BDNF) levels and postsynaptic densities were not different between experimental groups and no treatment effects were observed on hippocampal GSK3 $\beta$, tau phosphorylation or A $\beta$ levels. In conclusion, GEBR$7 \mathrm{~b}$ can enhance spatial memory function in the APPswe/PS1 $\triangle E 9$ mouse model of $A D$. Although the underlying mechanisms of its cognition-enhancing potential remain to be elucidated, PDE4D inhibition appears an interesting novel therapeutic option for cognitive deficits in $A D$. 


\section{Introduction}

Alzheimer's disease (AD) is a neurodegenerative disorder, clinically characterized by progressive memory loss and impairments in multiple cognitive domains, comprising activities of daily living. From a neuropathological perspective, AD hallmarks encompass extracellular amyloid- $\beta(A \beta)$ plaques and intracellular neurofibrillary tangles comprised of the protein tau. The loss of brain volume, known as atrophy, is often attributed to overt synapse loss. It has indeed been suggested that $A D$ is a synaptic disorder [658], as cognitive impairment has shown to be more robustly correlated with the degree of synapse loss than the other AD hallmarks [25]. Current treatment options have focused on enhancing cholinergic signaling, as acetylcholinesterase inhibitors (e.g. rivastigmine and donepezil) prevent the enzymatic breakdown of acetylcholine, or on decreasing glutamatergic excitotoxicity through antagonism of the N-methyl-D-aspartate (NMDA)-receptor (e.g. memantine) [53]. However, the efficacy of these drugs is limited and some induce gastrointestinal side effects [54]. Considering that $60-70 \%$ of the 35.6 million people worldwide suffering from dementia is caused by $A D$ [5], the need for novel therapeutic options is pending.

Phosphodiesterase inhibitors (PDE-Is) have received increasing attention as cognitive enhancers, by their ability to modulate levels of cyclic nucleotide, which play a key role in intracellular signal transduction cascades [657]. Of specific interest are PDE4-Is, as PDE4 is expressed abundantly in the brain, and is thought to play an important role in learning and memory processes [616, 659]. PDE4 can tightly regulate the level of cyclic adenosine monophosphate (CAMP), by selective hydrolysis of this cyclic nucleotide, and consequently also affect the activation of protein kinase $A$ (PKA) and phosphorylation of cAMP response element-binding (CREB) protein. As a transcription factor, phosphorylated CREB (pCREB) can regulate expression of many genes involved in signal transduction and cellular plasticity, e.g. brain-derived neurotrophic factor (BDNF). Consequently, inhibition of PDE4 can elevate the intracellular concentrations of cAMP, thereby enhancing this CAMP/PKA/CREB pathway, which has shown to play a pivotal role in the establishment of long-term potentiation (LTP); the proposed neurophysiological correlate of memory [59-61, 601, 602]. Indeed, the cognition-enhancing potential of PDE4-Is have been demonstrated in multiple behavioral tasks and across various species (for an extensive review, see [55]).

The efficacy of PDE4-Is in AD research has been established both in vitro as well as in vivo. By reducing PKA activity, the synaptotoxic $A \beta$ can inhibit formation of LTP $[61,660]$, which could be overcome by administrating the PDE4-I rolipram [61]. In vivo, both acute and chronic treatment of mouse models of $A D$ and intrahippocampal $A \beta$ injection rat models with rolipram could improve memory function by restoration of pCREB levels [65, 66, 610]. Additionally, 3-week rolipram administration could ameliorate AD-related alterations in dendrite and spine morphology [646]. Moreover, PDE-Is may regulate tau phosphorylation as tau contains many PKA phosphorylation sites [661]. Alternatively, PKA can phosphorylate 
and thereby activate or inhibit the kinase glycogen synthase kinase $3 \beta$ (GSK3 $\beta$ ), another key player in tau phosphorylation [662]. Indeed, a role for PDE4 has been established in the phosphorylation of tau in endothelial cells $[663,664]$, and may perform similar functions in neurons. Considering its wide array of intracellular effects both on signal transduction as well as AD-related pathology, PDE4-Is could be regarded as a suitable therapeutic strategy for the treatment of AD.

Unfortunately, most PDE4-Is, including rolipram, are aspecific and therefore target all 4 different gene products (PDE4A-D). However, the emetic side effects, probably through inhibition of PDE4D in particular [68, 612], have seriously hampered the clinical development of PDE4 inhibitors. In an attempt to solve these emetic side effects, small-molecule allosteric modulators of PDE4D were designed that do not completely inhibit enzymatic activity (Imax $\approx 80-90 \%$ ). Indeed, this approach reduced the potential to cause emesis [665]. Another interesting compound is GEBR-7b, which is a full inhibitor of PDE4D [523]. Compared to rolipram, GEBR-7b was 10 times more effective in improving memory performance in healthy rodents, yet its potential to induce emesis was reduced 100 times [523]. Neither these small-molecule allosteric modulating compounds nor GEBR-7b have so far been tested in an animal model of $A D$.

The aim of the current study was to investigate whether chronic treatment with GEBR$7 \mathrm{~b}$ could improve spatial, i.e. hippocampal, memory function in the APPswe/PS1 $\triangle E 9$ mouse model of $A D$, by restoring $C R E B$ phosphorylation, BDNF signaling and postsynaptic plasticity. Additionally, the effects of GEBR-7b treatment on AD-related neuropathology were assessed, including tau signaling pathways and $A \beta$ levels.

\section{Material and Methods}

\subsection{Animals}

All experimental animal research was executed according to protocols approved by the local Animal Ethical Committee of Maastricht University, Maastricht, the Netherlands (UM-DEC 2009-177) and met governmental guidelines. All animals were housed under a $12 \mathrm{~h}$ light/dark cycle (lights on at 7:00 A.M, unless specified otherwise) in standard Makrolon cages on sawdust bedding, placed in an individually ventilated cage (IVC) unit, regulating temperature (approximately $20^{\circ} \mathrm{C}$ ) and humidity (between $40-60 \%$ ). A radio, which was playing softly, provided background noise. Food and water was provided ad libitum throughout the study. To obtain offspring, virgin wild-type (WT) female C57BL/6NCr mice (Charles River, L'Arbresle, France) were mated with male APPswe/PS1 $\Delta \mathrm{E} 9$ mice (Jackson Laboratory, strain \#005864, Bar Harbor, ME, USA) at 8-12 weeks of age. The latter express mouse/human chimeric APP695, harboring the Swedish K694M/N595L mutation, as well as the PS1 gene with a deletion of exon 9 (PS1 $\triangle \mathrm{E} 9)$ under the mouse prion protein promoter [47], and were backcrossed to a C57BL/6 background for at least 8 generations. This mouse 
model displays $A \beta$ plaques from about 4 months of age and shows the first hippocampal functional deficits from 7 months of age onwards [50-52]. At postnatal day 8 (P8) male offspring was weighed and toe-clipped to provide permanent identification while harvesting tissue for genotyping. Genotyping was performed using PCR amplifications of genomic DNA. Two PCR reactions were performed: one for determining the presence of the APP mutation and the other for the presence of the PS1 mutation. Each reaction contained primers for the mouse prion protein promoter (sense: CCT CTT TGT GAC TAT GTG GAC TGA TGT; antisense: GTG GAT AAC CCC TCC CCC AGC CTA GAC C) and either primers for APP (sense: GAC TGA CCA CTC GAC CAG GTT CTG; antisense: CTT GTA AGT TGG ATT CTC ATA TCC) or PS1 (sense: AAT AGA GAA CGG CAG GAG CA; antisense: GCC ATG AGG GCA CTA ATC AT). The endogenous prion protein promoter produces a $750 \mathrm{bp}$ product, while transgene positive-samples produce bands at $450 \mathrm{bp}$ (APP) or $600 \mathrm{bp}$ (PS1). At P28 ( \pm 1 day), male pups were weaned and housed per 2 with same sex littermates. At approximately 3.5 months of age, mice were housed individually to avoid aggressive behavior among the mice. Weight of the offspring was monitored regularly throughout the experiment, serving as an indication of general wellbeing.

\subsection{Drug treatment}

At 19 weeks of age, APPswe/PS1 $1 \mathrm{E} 9$ and $\mathrm{WT}$ mice received a daily subcutaneous (s.c.) injection $(5 \mathrm{ml} / \mathrm{kg})$ for 3 weeks consecutively. For this purpose, a stock solution of the PDE4D-I GEBR-7b $(50 \mathrm{mg} / \mathrm{ml})$ was prepared by dissolving the compound in dimethylsulphoxide (DMSO) and was kept at $4^{\circ} \mathrm{C}$. This stock dilution was then further diluted in $0.5 \%$ methyl 2-hydroxyethyl cellulose, resulting in a dose of $0.001 \mathrm{mg} / \mathrm{kg}$, and keeping DMSO constant at $0.005 \%$. Vehicle injections consisted of a $0.5 \%$ methyl 2hydroxyethyl cellulose/0.005\% DMSO solution. Working solutions were freshly prepared on a daily basis. To assess any effects of the 3 week injection paradigm, a 'no injection' group was added. Subsequently, 6 experimental groups were formed: WT animals not receiving any injections (WT-no inj, $n=12$ ), WT animals receiving vehicle injections (WT-veh, $n=12$ ), WT animals receiving GEBR-7b (WT-GEBR-7b, $n=12$ ), APPswe/PS1 $\triangle E 9$ mice not receiving any injections (AD-no inj, $n=12$ ), APPswe/PS1 $\triangle E 9$ mice receiving vehicle injections (ADveh, $n=13$ ), and APPswe/PS1 $\triangle E 9$ mice receiving GEBR-7b (AD-GEBR-7b, n=14). To exclude stress of daily handling from injection stress, all animals within the no-injection group were also handled on a daily basis.

\subsection{Behavioral testing}

For a global timeline of the behavioral tests conducted in this experiment, see Figure 1. Experimental groups without daily injections were included to control for possible adverse effects of chronic injections stress $[666,667]$. One day after the last injection the mice were subjected to the sucrose intake test (SIT) and the elevated zero maze (EZM), to assess the effects on affective behavior. Afterwards, mice were subjected to the open field (OF) test to 
assess locomotor behavior and to the Y-maze spontaneous alternation test (Y-maze) to measure working memory function. Three weeks later, at 26 weeks of age, mice were habituated for the object location task (OLT) and subsequently tested to measure long-term spatial memory. Following the OLT, the forced swim test (FST) was conducted as a measure of depressive-like behavior.

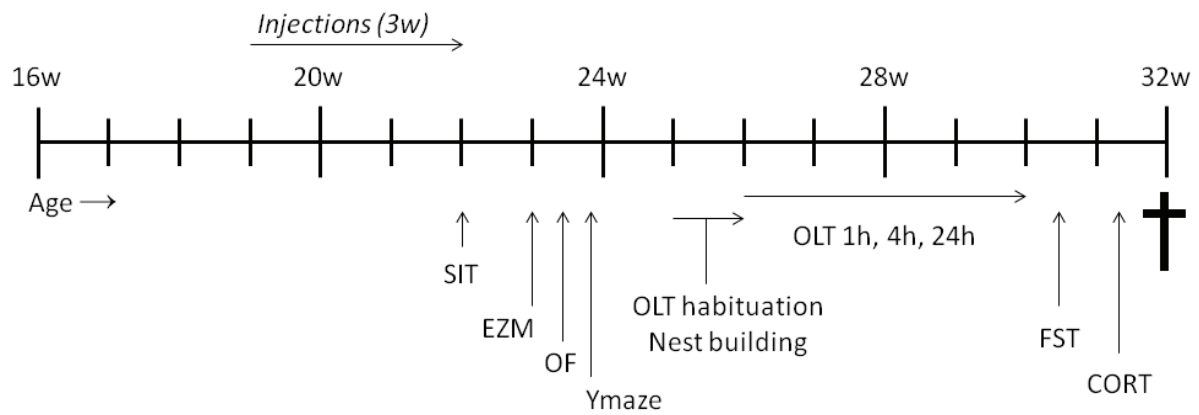

Figure 1: Global timeline of the experiment in weeks of age (w). Chronic treatment with the PDE4D-I GEBR-7b, vehicle or no injections started at $19 \mathrm{w}$, and continued for 3 weeks (daily, s.c.). The sucrose intake test (SIT) was performed one day after the last injection. In the week thereafter, the elevated zero maze (EZM), open field (OF) and Y-maze spontaneous alternation test were performed. At $25 \mathrm{w}$, mice were habituated for the object location task (OLT) and subsequently tested. Nest building behavior was assessed overnight. The forced swim test (FST) was performed at $30 \mathrm{w}$, followed by blood collection at $31 \mathrm{w}$ for corticosterone (CORT) determination, and mice were sacrificed at $32 \mathrm{w}$.

\subsection{1. $S I T$}

The SIT is often used to measure anhedonia, one of the core symptoms of depressive behavior $[668,669]$. Mice were first habituated to the $1 \%$ sucrose solution, by replacing their drinking water with the sucrose solution for 24 hours. On the day of the SIT, mice were deprived of food and water for 6 hours (starting at 7 AM), after which they received a bottle of $1 \%$ sucrose solution for 1 hour. Sucrose intake and general water and sucrose consumption were recorded by weighing the bottles before and after the intake. Sucrose intake was corrected for body weight.

\subsection{2. $E Z M$}

The EZM is an effective tool to measure anxiety in a rodent [527]. The apparatus consisted of a circular runway (diameter $46 \mathrm{~cm}$, width $5.5 \mathrm{~cm}$ ) made of black plastic material which was transparent for infrared light and elevated $20 \mathrm{~cm}$ above the floor [670]. The arena was divided into 4 quadrants: 2 opposing open quadrants with a border (height $3 \mathrm{~mm}$ ) to prevent the mouse from stepping down, and 2 opposing closed quadrants with side walls (height 20 $\mathrm{cm}$ ). Each mouse was placed individually into one of the open arms close to and facing a closed arm, and was allowed to explore the arena for 5 min under almost dark conditions. 
After each session the maze was thoroughly cleaned using a $70 \%$ ethanol solution. The movements of each mouse were tracked automatically using an infrared camera and Ethovision Pro (Noldus, The Netherlands) [528, 590]. Parameters for anxious behavior were the total distance traveled and percentage of time spent in the closed arms, corrected for possible freezing behavior at the start of the experiment (i.e. the latency to enter one of the closed arm).

\subsection{3. $O F$}

The open field arena is used to assess locomotor behavior [671]. The apparatus was made of acrylic and consisted of a grey square arena $(50 \times 50 \times 25 \mathrm{~cm})$, subdivided by grey walls (height: $25 \mathrm{~cm}$ ) into 4 separate arenas of $25 \times 25 \times 25 \mathrm{~cm}$, leaving the top open. One mouse was introduced per arena, thereby testing 4 mice simultaneously. After each session the arena was thoroughly cleaned using a $70 \%$ ethanol solution. The mouse was placed in the centre of the arena, being able to move around freely for $20 \mathrm{~min}$. The movements of each mouse were tracked automatically by using a video camera and Ethovision Pro (Noldus, The Netherlands). The total distance moved within 20 minutes was used as a measure of locomotor behavior.

\subsubsection{Y-maze}

Spatial working memory was assessed by using the Y-maze spontaneous alternation test, as previously described [524]. The symmetrical grey $\mathrm{Y}$-maze made of acrylic consisted of 3 arms, with all arms being apart from the others at a $120^{\circ}$ angle. Each arm was $40 \mathrm{~cm}$ long, $17 \mathrm{~cm}$ high, $4 \mathrm{~cm}$ wide at the bottom and $13 \mathrm{~cm}$ wide at the top. Each mouse was placed in the centre of the $Y$-maze and was free to explore the arena for $6 \mathrm{~min}$. The number of entries was counted per mouse: an entry required that both hind paws of the animal had to be placed completely inside the arm. A mouse would be making a triad when it visited all 3 arms consecutively. Between sessions, the maze was cleaned thoroughly with a $70 \%$ ethanol solution. As a measure for working memory, the percentage of alternations that the mouse made was calculated, being the number of triads divided by the maximum possible alternations (i.e. the total number of entries minus 2) x 100. If a mouse scored significantly above $50 \%$ alternations (the chance level for choosing the unfamiliar arm), this was indicative of functional working memory.

\subsubsection{OLT}

Long-term spatial memory was tested using the OLT, as described previously [522]. In brief, the setup consisted of a circular arena, $43 \mathrm{~cm}$ in diameter. The $40 \mathrm{~cm}$ high wall was divided into two halves, one made of white polyvinyl chloride, the other made of transparent polyvinyl chloride. Two objects were placed symmetrically in the middle of the arena. Four different sets of objects were used, and each object was available in triplicate. The different objects were: 1) a massive metal cube $(2.5 \times 5 \times 7.5 \mathrm{~cm})$ with 2 holes (diameter $1.5 \mathrm{~cm}), 2)$ a 
white plastic cube with rounded off edges placed on a small stand of the same material (maximal diameter $3.5 \mathrm{~cm}$ and total height $11.5 \mathrm{~cm}$ ), 3) a brass cylinder with a tapering top (maximal diameter $3 \mathrm{~cm}$ and total height $11.5 \mathrm{~cm}$ ) and 4) a massive aluminum cube with a tapering top $(4.5 \times 4.5 \times 8.5 \mathrm{~cm})$. The objects could not be displaced by the mouse. The task was conducted as previously described $[522,523]$. To habituate the animals to behavioral testing, all mice were first handled, weighed and were able to explore the empty arena of the OLT for 4 minutes. Subsequently, the animals were habituated to the $1 \mathrm{~h}$ and $4 \mathrm{~h}$ interval of the OLT. A testing session consisted of 2 trials, with each trial lasting 4 minutes. During the first trial (T1) the arena contained 2 identical objects at fixed locations. A mouse was introduced into the arena by placing it with its nose to the middle of the transparent segment of the wall. After the first exploration period had elapsed, the mouse was placed back in its home cage. Subsequently, after a predetermined time interval (e.g. $1 \mathrm{~h}$ or $4 \mathrm{~h}$ ) the mouse was put back into the arena for the second trial (T2). However, now, one of the objects had shifted location, which was randomized in a balanced manner over the groups and the different sessions to reduce potential bias due to preference for particular objects. The time spent exploring the objects during T1 and T2 was recorded manually using a computer. Exploring behavior was defined as follows: directing its nose to the object at a distance of no more than $1 \mathrm{~cm}$ and/or touching the object with its nose. Sitting on the object was not considered exploration. In order to avoid the presence of olfactory cues, all objects and the arena were cleaned thoroughly with ethanol between sessions. In order to determine whether a mouse had remembered the familiar location of the object, several measures were used. The amount of exploration time is recorded in T1 (named e1) and T2 (e2). The discrimination index $d 2$ calculates whether the mouse spends more time at the novel location while correcting for the total e2: (exploration time new location - exploration time old location)/e2. The latter will be an indication of recognition of the novel location of the objects, i.e. spatial memory. A minimum of 1.5 seconds exploration was set as an inclusion criterion to reliable measure memory performance [590].

\subsubsection{Nesting behavior}

Nesting behavior is dependent on hippocampal functioning [672, 673], and is part of the normal cage behavior in a mouse (activities of daily life [674]). Gross differences in nestbuilding were assessed as described previously [675]. In short, the mice were provided with equal pieces of paper towel. After $24 \mathrm{~h}$, the quality of the nest was assessed; scoring 0 when no nest was produced and 1 if a nest was constructed. A nest was defined as follows: the tissue was shredded and manipulated into a nest to provide the mouse with warmth and shelter.

\subsubsection{FST}

The FST is a widely used behavioral task to study depressive-like behavior in rodents [529]. Under low light conditions, each mouse was individually placed into a perspex cylinder 
(diameter $17 \mathrm{~cm}$, height $40 \mathrm{~cm}$ ) filled with $30^{\circ} \mathrm{C}\left(+/-2^{\circ} \mathrm{C}\right)$ water to a height of $15 \mathrm{~cm}$ for 6 min. The total distance swum was used as a measure of depressive-like behavior, i.e. a lesser distance swum being indicative of heightened despair [510]. Each session was scored automatically with Ethovision Pro (Noldus, The Netherlands) [590].

\subsection{Blood sampling and corticosterone radioimmunoassay}

In order to determine hypothalamo-pituitary-adrenal (HPA) axis responsiveness in the face of a stressor, blood samples were taken to determine corticosterone concentrations in these mice. First, the mice were taken out of their home cage, immediately after which a blood sample was drawn from the vena saphena (basal corticosterone concentration) using heparinized blood collection tubes (Microvette ${ }^{\circledR}$ CB300, Sarstedt, Germany). Subsequently, the mouse was restrained in a glass cylinder to induce a stress response. After 20 min of restraint stress, another blood sample was drawn from the vena saphena (stress-induced corticosterone concentration). Animals were then placed back in their home cage and housing room and allowed to recover for $60 \mathrm{~min}$ after which a third sample was taken ('recovery' corticosterone concentration). All blood samples were kept on ice and subsequently centrifuged at $3000 \mathrm{rpm}$ for $10 \mathrm{~min}$ at $4^{\circ} \mathrm{C}$, after which plasma was isolated, frozen down to $-80^{\circ} \mathrm{C}$ and stored until further processing. For the corticosterone radioimmunoassay, an ImmuChem ${ }^{\mathrm{TM}}$ Double Antibody Corticosterone ${ }^{125}$ I RIA Kit for rodents (MP Biomedicals, Orangeburg, NY, USA) was used. The assay was performed according to the manufacturer's instructions. Briefly, for each sample, $5 \mu$ l serum was diluted in steroid diluents (1:100 for basal and recovery samples and 1:200 for stress samples). Per $50 \mu \mathrm{l}$ of diluted sample, $50 \mu \mathrm{l}$ of corticosterone ${ }^{-125} \mathrm{I}$ and $100 \mu \mathrm{l}$ anti-corticosterone was added, and samples were incubated at room temperature (RT) for $2 \mathrm{~h}$. Afterwards, $250 \mu \mathrm{l}$ precipitant solution was added and tubes were centrifuged at $2300 \mathrm{rpm}$ for $15 \mathrm{~min}$. Supernatant was aspirated and the precipitate was counted in a Wizard Gamma Counter 2470 (Perkin Elmer, Waltham, MA, USA).

\subsection{Tissue preparation}

At 32 weeks of age, all mice were sacrificed by decapitation, and the whole hippocampus was isolated by microdissection. Hippocampal tissue was snap frozen in liquid nitrogen and homogenized using a Mini-Bead Beater (Biospec products, Bartlesville, USA), 3 times for 30 seconds in $0.5 \mathrm{ml}$ homogenisation buffer (100 mM Tris, $200 \mathrm{mM} \mathrm{NaCl}, 1 \mathrm{mM}$ EDTA, $2 \mathrm{mM}$ DTT, $0.05 \%$ Triton X-100, one complete protease inhibitor tablet (Roche \#11836153001, Almere, The Netherlands) per $20 \mathrm{ml}$ buffer, and 1 tablet of PhosSTOP phosphatase inhibitor cocktail tablets (Roche, \#04906845001, Almere, The Netherlands) per $10 \mathrm{ml}$ buffer), while storing the samples ice for 5 minutes in between each homogenization bout. Samples were centrifuged for $20 \mathrm{~min}$ at $4^{\circ} \mathrm{C}$ at $16000 \mathrm{RCF}$, and the supernatant was stored at $-80^{\circ} \mathrm{C}$. Protein concentrations were determined using the Bio-Rad Lowry Protein Assay (Bio-Rad Laboratories Inc., Hercules, USA). 


\subsection{Western Blotting}

Protein levels were assessed of CREB, pCREB, BDNF, the BDNF receptors tropomyosinrelated kinase $B\left(\right.$ TrkB) and $p 75$ neurotrophin receptor $\left(p 75^{N T R}\right), G S K 3 \beta, p G S K 3 \beta$ at serine 9 (pGSK-Ser9), total tau, pTau at serine 396 (pTau-Ser396), and postsynaptic density 95 (PDS95) as a marker for postsynaptic plasticity. Hippocampal homogenates in sample buffer were boiled for 5 minutes and then separated on a 14\% (BDNF), 12\% (TrkB) or 10\% (all

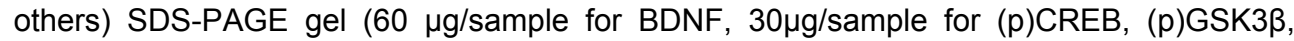
$\mathrm{p} 75^{\mathrm{NTR}}$ and TrkB, 25 $\mathrm{g} / \mathrm{sample}$ for pTau and PSD95, $10 \mu \mathrm{g} / \mathrm{sample}$ for Tau). Following electrophoresis, proteins were transferred to a nitrocellulose membrane (Bio-Rad Laboratories, Hercules, USA) which was previously blocked with blocking buffer $(50 \%$ Odyssey blocking buffer in PBS, Li-Cor, Lincoln, USA) for one hour at room temperature. Next, the membranes were incubated overnight at $4^{\circ} \mathrm{C}$ with the primary antibodies in blocking buffer: 1:3000 mouse anti-CREB (Cell Signaling Technology \#9104, Beverly, USA), 1:100 rabbit anti-pCREB (Cell Signaling Technology \#9198), 1:600 rabbit anti-BDNF $(\mathrm{H}-117$; Santa Cruz Biotechnology \#SC-20981, Heidelberg, Germany), 1:250 rabbit anti-TrkB (Cell Signaling Technology \#4606), 1:1000 rabbit anti-p75 NTR (Millipore \#07-476, Billerica, MA), 1:2000 mouse anti-PSD95 (QED Bioscience, San Diego, CA, USA), 1:500 rabbit monoclonal anti-pGSK3 $\beta$ Ser9 (Cell signalling technology \#9336S), 1:1000 rabbit monoclonal antiGSK3 $\beta$ (Cell signalling technology \#9315S), 1:10,000 mouse monoclonal anti-tau (Cell signalling technology \#4019), 1:1000 mouse monoclonal anti-ptau S396 (Cell signalling technology \#9632S), with 1:1000 mouse anti- $\beta$-actin (Santa Cruz Biotechnology \#SC-81178) for normalization. After washing with phosphate-buffered saline-0.1\%Tween (PBS-T), membranes were incubated for 1 hour at room temperature with secondary antibodies in blocking buffer: 1:5000 goat anti-rabbit IRDye 800 (\#926-32211, Li-Cor, ) and 1:5000 donkey anti-mouse IRDye 680 (\#926-32222, Li-Cor). Membranes were washed in PBS-T and fluorescent bands were visualized using an Odyssey Infrared Imaging System (Li-Cor). Intensities of specific bands were quantified using ImageJ (http://rsbweb.nih.gov/ij/), corrected for background signal and $\beta$-actin signal. Within the BDNF blot different BDNF domains were analyzed, distinguishing between mBDNF (14 kDa) and unglycosylated and glycosylated proBDNF (16 and $18 \mathrm{kDa}$, respectively), as previously described [676].

\subsection{ELISA $A \beta 40+42$}

Concentrations of hippocampal $A \beta 40$ and $A \beta 42$ were determined in the hippocampal homogenates using ELISA kits against human A 40 and Aß42 (Invitrogen, \#KHB3481 and \#KHB3544, Camarillo, USA), following the manufacturer's protocol. In addition, the $A \beta 42 / A \beta 40$ ratio was determined for each group.

\subsection{Statistical analysis}

All data are presented as mean and standard error of the mean (SEM). Two-way analysis of variance (ANOVA) was performed for comparison between groups accounting for the main 
and interactive effects of genotype (WT, APPswe/PS1 $\triangle E 9$ ) and treatment (no injection, vehicle, PDE4D-I). Repeated measures ANOVA were performed to assess OLT D2 scores over the different intervals, as well as for the corticosterone secretion (LN-transformed) over time. As indications for memory performance, a one-sample t-test was performed for the $Y$ maze spontaneous alternation test and the OLT, with testing values 50 and 0 , respectively. Statistical assessment of nest building behavior was performed with the Pearson $x^{2}$ test. Statistical significance was set at an $\alpha$ level of 0.05 and all tests were two-tailed. All statistical analyses were conducted using the Statistical Package for the Social Sciences (SPSS 15.0, SPSS Inc., Chicago, IL, USA). All graphs were constructed in GraphPad Prism (version 4, GraphPad Software, San Diego, CA, USA).

\section{Results}

\subsection{Behavioral results}

\subsubsection{Weight monitoring}

For an evaluation of general wellbeing, weights were monitored regularly throughout the experiment. Repeated measures ANOVA indicated a significant time effect $(F(2.8$, $166.4)=2599.70, p<0.001$ ), but no significant effects of time* genotype, time*treatment or time* genotype*treatment, indicating that animals gained weight over time but this weight gain was equal for all groups (data no shown).

\subsection{2. $S I T$}

Results of the $1 \mathrm{~h}$ sucrose intake showed no effects of genotype, treatment or genotype*treatment (see Figure 2). It was noted that APPswe/PS1 $\triangle \mathrm{E} 9$ mice lost significantly more weight during the $6 \mathrm{~h}$ fasting period prior to the actual SIT, as a significant genotype effect was found $(F(1,74)=12.06, p<0.01)$, but no effects for treatment or genotype*treatment (see Table 1).

\subsection{3. $E Z M$}

Significant genotype effects were observed for the percentage of time spent in the closed arms of the EZM $(F(1,74)=4.13, p<0.05)$ with APPswe/PS1 $\triangle E 9$ mice spending more time in the closed compartments than WT mice, indicative of heightened anxiety (see Figure 2). Moreover, significant genotype effects were found for the total distance moved in the EZM $(F(1,74)=11.58, p<0.05)$, with APPswe/PS1 $\triangle E 9$ mice covering significantly more distance (see Table 1). No significant effects were found for treatment or genotype ${ }^{\star}$ treatment in time spent in the closed arm or the total distance moved. 
A

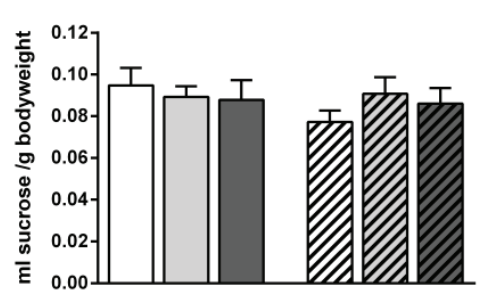

C

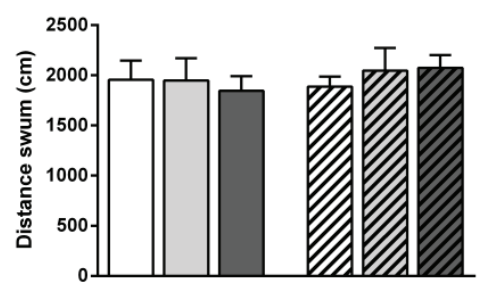

B

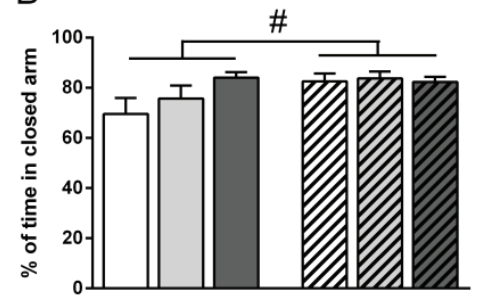

$\mathrm{D}$

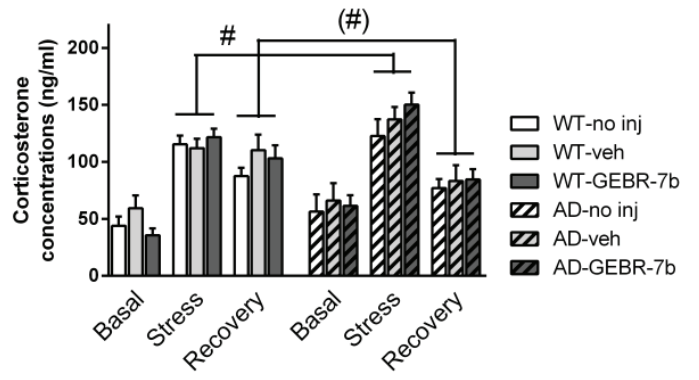

Figure 2: Assessment of affective behavior and HPA axis reactivity. Bars represent mean and S.E.M per group A: Amount of sucrose consumed in 1 hour, corrected for body weight in the sucrose intake test (SIT). No differences were found between the groups. B: Percentage of time spent in the closed arms of the elevated zero maze (EZM). APPswe/PS1 $\triangle E 9$ mice spent significantly more time in the closed arms, indicative of more anxiety. C: Distance swum in the forced swim test (FST). No differences were found in terms of depressive-like behavior between the groups. D: Plasma corticosterone concentrations during basal, stressful, and recovery conditions. APPswe/PS1 $\triangle \mathrm{E} 9$ mice secreted significantly more corticosterone after being stressed, while tended to have a lower plasma corticosterone concentration during the recovery condition. \#: significant genotype effect, $p<0.05$; (\#): trend for genotype effect, $p=0.078$.

\subsubsection{OF}

Locomotor assessment in the OF demonstrated a significant genotype effect $(F(1$, $74)=32.18, p<0.001)$, as APPswe/PS1 $\triangle E 9$ mice traveled a greater distance in 20 min when compared to WT mice. Treatment or genotype*treatment did not affect the total distance moved (see Table 1).

\subsubsection{Y-maze spontaneous alternation}

When comparing the percentage of alternations per group to the chance level of $50 \%$, it was shown that every group performed significantly better than chance level $(t(>11)>3.26$, $p<0.01$ ) (see Figure 3), indicative of functional working memory. No significant effects of genotype, treatment or genotype*treatment could be observed for the percentage of 
alternations. Genotype did, however, significantly affect the number of entries within the $Y$ maze $(F(1,74)=16.69, p<0.001)$, with APPswe/PS1 $\triangle E 9$ mice making more entries. Treatment and genotype*treatment did not affect the number of entries (see Table 1).

\subsection{6. $O L T$}

Repeated measures ANOVA on exploration times in the $1 \mathrm{~h}, 4 \mathrm{~h}$, and $24 \mathrm{~h}$ interval of the OLT showed an overall effect of genotype $(F(1,68)=13.88, p<0.01)$, with APPswe/PS1 $\triangle E 9$ mice having higher exploration times than WT mice. No effects of treatment or genotype*treatment on exploration times were observed (see Table 1).

Repeated measures ANOVA on the D2 scores within the $1 \mathrm{~h}, 4 \mathrm{~h}$, and $24 \mathrm{~h}$ interval of the OLT showed a significant effect of interval $(F(1.8,123.5)=8.31, p<0.001)$, but not of interval ${ }^{*}$ genotype, interval*treatment or interval* ${ }^{*}$ enotype*treatment (data not shown). Overall, a significant genotype effect was observed $(F(1,68)=11.13, p<0.01)$, as APPswe/PS1 1 E9 mice had lower D2 scores than WT mice. No effects were found for treatment or genotype*treatment. Analysis of the different intervals indicated that this overall genotype effect could be mainly explained by the $4 \mathrm{~h}$ interval, as no effects of genotype, treatment or genotype*treatment could be observed in the $1 \mathrm{~h}$ and $24 \mathrm{~h}$ interval. At the $4 \mathrm{~h}$ interval, a significant genotype effect was demonstrated $(F(1,73)=6.99, p<0.05)$, with APPswe/PS1 $\triangle E 9$ mice performing worse, although no overall effects treatment or genotype*treatment were observed. Finally, to examine in detail at the $4 \mathrm{~h}$ interval which experimental groups could significantly distinguish the familiar location of the object from the novel location [677], it was demonstrated that all WT groups could distinguish between the familiar and the novel location $(t(11)>3.68, p<0.01)$. The AD-no inj and AD-veh group were unable to distinguish between the familiar and novel location, whereas the AD-PDE4D group still remembered the familiar location $(t(13)=4.97, p<0.01)$ (see Figure 3 ).

\subsubsection{Nest building behavior}

The $x^{2}$ test demonstrated that the odds for building a nest when having the WT genotype tended to be higher than for the APP/PS1 genotype $\left(x^{2}(1)=3.65, p=0.056\right)$. Treatment did not significantly affect nest building behavior $\left(X^{2}(2)=2.22\right.$, n.s.) (see Figure 3 ).

\subsubsection{FST}

The total distance swum in the FST was not affected by genotype, treatment or genotype*treatment (see Figure 2). 
A

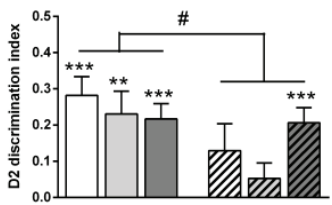

B

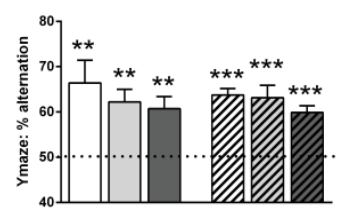

C

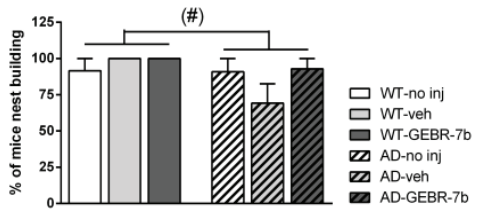

Figure 3: Hippocampus-dependent behavior as tested with the object location task (OLT) at the 4h interval (A), the $Y$-maze spontaneous alternation test (B) and nest building behavior (C). Bars represent mean and S.E.M per group. A: APPswe/PS1 $1 \mathrm{E} 9$ mice generally scored worse than WT mice. When comparing the D2 scores with 0 (no memory), it was shown that all WT mice could sufficiently distinguish the old from the novel location, while AD-no inj and AD-s.c. mice could not. However, when APPswe/PS1 $\triangle E 9$ mice were previously treated with the PDE4D-I, they were able to significantly distinguish the novel from the old location. B: all mice showed intact spatial working memory function, as they all performed significantly better than the $50 \%$ chance level. C: Nest building behavior tended to be decreased in APPswe/PS1 $\triangle E 9$ mice. \#: significant genotype effect, $p<0.05$; (\#): trend for genotype effect, $p=0.056$; asterisks: significantly different compared to $0(A)$ or $50 \%(B){ }^{* * *}: p<0.001,{ }^{* *}: p<0.01$.

\subsection{Plasma corticosterone concentrations}

Repeated measures ANOVA on plasma corticosterone concentrations during basal, stressful and recovery conditions revealed a significant condition $(F(1.49,93.85)=78.96$, $p<0.01)$ and a trend for a condition*genotype effect $(F(1.49,93.85)=2.94, p=0.056)$, but no effects of condition*treatment or condition*genotype*treatment. Two-way ANOVA of the different conditions revealed no effects of genotype, treatment or genotype*treatment on basal corticosterone secretion. Stress-induced corticosterone secretion was significantly affected by genotype $(F(1,70)=4.74, p<0.05)$, as APPswe/PS1 $\Delta E 9$ mice had higher corticosterone levels, but not by treatment or genotype*treatment. Recovery corticosterone concentrations tended to be affected by genotype $(F(1,69)=3.17, p=0.079)$, but not by treatment or genotype*treatment, with APPswe/PS1 $\mathrm{E} 9$ mice having higher corticosterone secretion (see Figure 2).

\subsection{Hippocampal protein assessment}

\subsection{1. (p)CREB}

Hippocampal pCREB concentrations were significantly affected by genotype $(F(1,39)=8.98$, $\mathrm{p}<0.01$ ), with APPswe/PS1 $\triangle \mathrm{E} 9$ mice having lower concentrations, but not by treatment or genotype*treatment. CREB concentrations were unaffected by genotype, treatment or genotype*treatment (see Figure 4). 

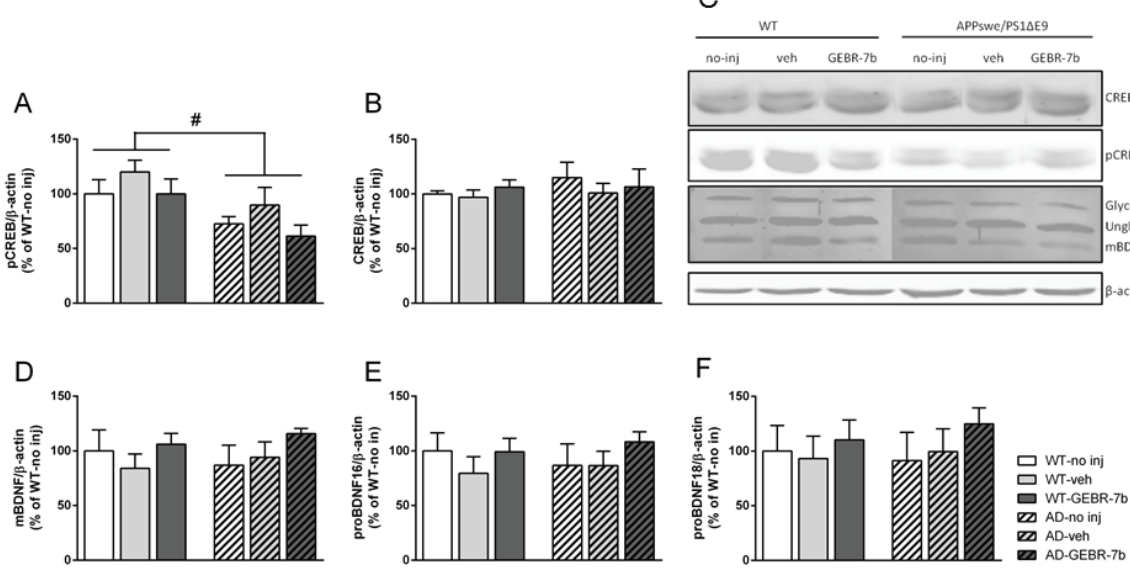

Figure 4: Levels of hippocampal cAMP regulated element-binding protein (CREB), phosphorylated CREB (pCREB), mature brain-derived neurotrophic factor (mBDNF) and proBDNF as measured by western blot. Bars represent mean and S.E.M per group ( $n=5-8)$. A: APPswe/PS1 $\triangle E 9$ mice show significantly lower concentrations of pCREB than WT mice. B: No differences in total CREB levels were observed between the groups. C: Representative western blots of the investigated proteins. D-F: Levels of mBDNF, unglycosylated proBDNF (16 kDa) and glycosylated proBDNF (18 kDa) were not different among the groups. \#: significant genotype effect, $p<0.05$.

\subsection{2. $B D N F$}

No differences between groups were observed in BDNF, unglycosylated proBDNF or glycosylated proBDNF as a consequence of genotype, treatment or genotype*treatment (see Figure 4).

\subsubsection{BDNF receptors}

Genotype, treatment or genotype*treatment did not affect concentrations of the BDNF receptor TrkB, its truncated isoforms, TrkB.T1, or the receptor $\mathrm{p} 75^{\mathrm{NTR}}$ (data not shown).

\subsubsection{PSD95}

PSD95 levels were not altered by genotype, treatment or genotype*treatment (data not shown).

\subsubsection{Tau signaling}

No significant differences were observed between the groups in the level of GSK3 $\beta$ or pGSK3 $\beta$-Ser9. Similarly, genotype, treatment or genotype*treatment did not alter levels of pTau-Ser396. Alternatively, total tau levels tended to be affected by genotype $(F(1$, $38)=3.64, p=0.065$ ), yet not by treatment or genotype*treatment, with APPswe/PS1 $\triangle E 9$ mice having significantly higher levels of total Tau (see Figure 5). 
A

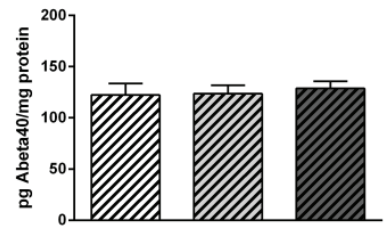

C

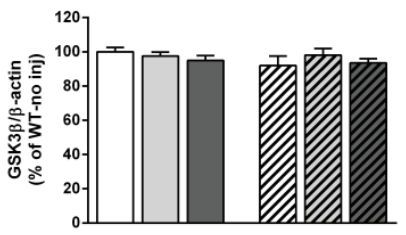

$\mathrm{E}$

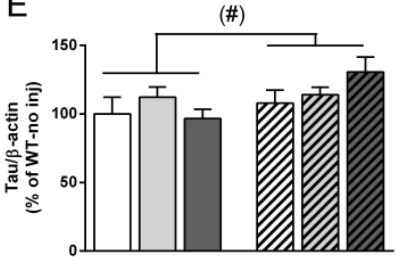

B

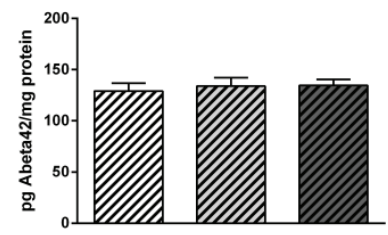

D

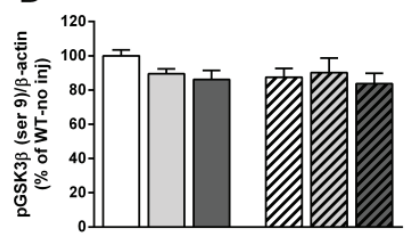

$\mathrm{F}$

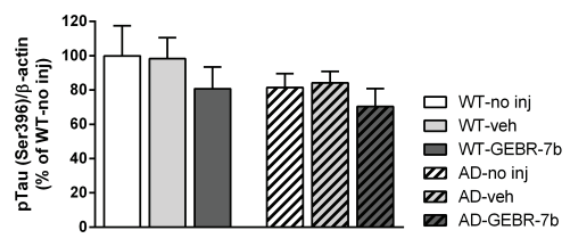

$G$

WT APPswe/PS1dE9
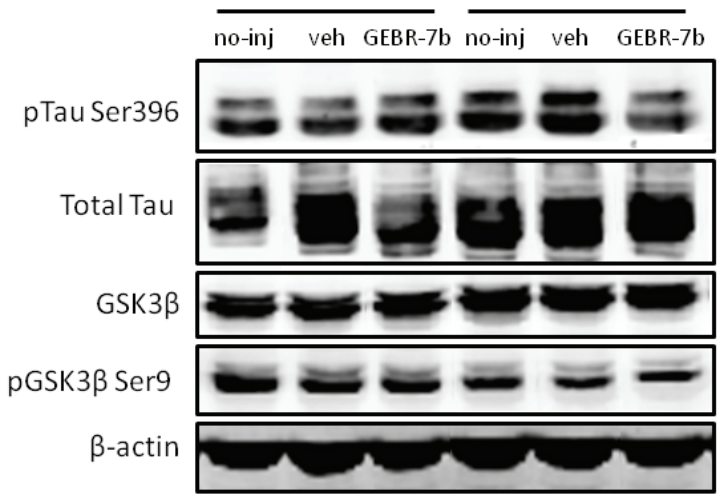

Figure 5: Assessment of soluble $A \beta$ and tau signaling. Bars represent mean and S.E.M per group $(n=5-$ 8). $A+B$ : As soluble $A \beta 40$ (A) and $A \beta 42$ (B) fractions cannot be detected in WT mice, only APPswe/PS1 $\triangle E 9$ mice were assessed. Treatment did not alter $A \beta$ concentrations in the hippocampus as measured with ELISA. C+D: levels of glycogen synthase kinase $3 \beta$ (GSK3 $\beta ; C)$ and phosphorylated GSK3 $\beta$ at serine 9 (pGSK3 $\beta$; D) were not different between the groups. E: APPswe/PS1 $\triangle E 9$ mice tend to have higher levels of tau protein than WT mice. F: No differences were observed in concentrations of phosphorylated tau at serine 396 (pTau). G: Representative western blots of the different proteins investigated. (\#): trend for genotype effect, $p=0.065$. 


\subsection{Soluble $A \beta$ analysis}

Concentrations of soluble $A \beta 40$ and $A \beta 42$ in the hippocampus of APPswe/PS1 $\triangle E 9$ mice remained unaltered by treatment (see Figure 5).

\section{Discussion}

The main aim of this study was to investigate the cognition-enhancing potential of GEBR-7b in a mouse model of $A D$. The results indicate that 3 weeks of GEBR-7b treatment enhanced long-term spatial memory function in 7-month-old APPswe/PS1 $\triangle \mathrm{E} 9$ mice, independently of structural upregulation of pCREB, BDNF or PSD95, as a marker for postsynaptic densities. Additionally, GEBR-7b treatment did not alter AD-related neuropathology.

\subsection{Chronic GEBR-7b treatment improves hippocampus-dependent memory function in APPswe/PS1 $1 E 9$ mice}

This study corroborates previous findings that, at 7 months of age, APPswe/PS1 $\triangle E 9$ mice display impairments in hippocampus-dependent behavior, as APPswe/PS1 $\triangle E 9$ mice performed significantly worse at the $4 \mathrm{~h}$ interval of the OLT when compared to WT mice [52, 678]. WT mice could all distinguish the familiar from the novel location at the $4 \mathrm{~h}$ interval, irrespective of treatment, indicating that chronic PDE-I treatment has no effect in WT animals, as was previously also shown in other studies [66, 67, 632]. Yet, the same 3-week GEBR-7b treatment at 5 months of age had a long-lasting prophylactic effect on AD-related spatial memory deficits at the $4 \mathrm{~h}$ interval of the OLT in APPswe/PS1 $\triangle E 9$ mice, even though hippocampal plaques are starting to deposit at that age [50]. Hippocampal silencing or knock-out of the PDE4D gene produced similar cognition-enhancing effects as GEBR-7b treatment [679]. However, as gene-therapy is currently not yet applicable, treatment with a PDE4-I may be a more feasible and convenient way to improve cognition in patient groups.

Nest building behavior is also dependent on intact hippocampal functioning [672, 673], and tended to be impaired in APPswe/PS1 $1 \mathrm{E} 9$ mice, as nests were built less frequently by APPswe/PS1 $1 \mathrm{E} 9$ compared to WT mice. Spatial working memory function, as assessed by the Y-maze spontaneous alternation test and known to be dependent on hippocampal as well as frontal cortex function [680], was not different between the APPswe/PS1 $\triangle E 9$ and WT mice, and performance was not further improved by chronic GEBR-7b treatment. Indeed, ameliorations in working memory performance by PDE-Is were only reported in animals otherwise presenting memory deficits $[66,67]$.

\subsection{APPswe/PS1 1 E9 mice display heightened levels of anxiety, locomotor activity and altered HPA axis response}

APPswe/PS1 $1 \mathrm{E} 9$ mice spent more time in the closed compartments of the EZM, which corroborates previous findings of heightened anxiety levels in APPswe/PS1 $1 \mathrm{E} 9$ mice compared to WT mice [681]. GEBR-7b treatment could not alter this phenotype, which is in 
line with findings by $\mathrm{Li}$ and colleagues, showing that knocking-out PDE4D does not affect levels of anxiety [679]. Of note, the total distance moved in the EZM was significantly higher in APPswe/PS1 $1 \mathrm{E} 9$ mice than in WT mice, suggestive of increased locomotor activity. Similarly, it was demonstrated in the OF that APPswe/PS1 $1 \mathrm{E} 9$ mice were hyperactive, as they traveled greater distances compared to WT mice, within the same timeframe. Heightened activity was also observed in the Y-maze and the OLT, with APPswe/PS1 $1 \mathrm{E} 9$ mice making more entries and having higher exploration times, respectively, which is in line our previous findings [678]. A possible explanation could lie in AD-related damage to hippocampal CA and DG subregions, as this has been associated with a hyperactive phenotype [682]. Hyperactivity is also observed in AD patients [683, 684], as well as in other mouse models of $A D[284,685,686]$. Depressive-like behavior, as measured by the degree of anhedonia and learned helplessness in the SIT and FST, respectively, was not significantly different between the groups. On the other hand, the current study substantiated our previous finding that the HPA axis is dysregulated in APPswe/PS1 $\Delta$ E9 mice [678], as higher secretion of corticosterone in response to an acute stressor, and a tendency to faster recovery was observed in APPswe/PS1 $\triangle E 9$ mice compared to WT mice. AD patients are also inflicted with HPA axis dysfunction [107] and this phenomenon is prevalent in other mouse models of $A D$ as well $[620,621,631]$.

Of note, by adding a no-injection group, we aimed to control for long-term effects of injection stress on affective behavior and neuroendocrine regulation. As no significant differences were found between the no-injection group and the vehicle-treated group, it can be concluded that 3 weeks of s.c. injections had no long-term impact on affective behavior or HPA axis function. Although PDE4D knock-out has been implicated in the regulation of antidepressant behavior [687], GEBR-7b by itself also did not have a long-term effect on affective behavior, indicating that as a therapeutic target, GEBR-7b does not stretch beyond enhancement of cognition.

\subsection{GEBR-7b treatment does not alter pCREB, BDNF levels or postsynaptic densities}

PDE-Is are thought to exert their cognition-enhancing effects via activation of pCREB and subsequent expression of downstream targets, like BDNF. Our data demonstrate that at 8 months of age APPswe/PS1 $\triangle E 9$ mice had significantly less PCREB than WT mice, which is in concordance with previous reports [639, 641], other mouse models of $A D[66,638,688]$ and with $A \beta$-injected rat models $[610,611]$. As $A \beta$ is known to interfere with CREB activity, possibly via inhibition of intracellular kinases such as PKA and ERK $[61,637,640,688]$, it is most likely that the age-related increase in A $\beta$ production in APPswe/PS1 $\triangle E 9$ mice [50] has led to this decrease in pCREB. Surprisingly, pCREB levels were not changed as a result of chronic GEBR-7b treatment, despite the observed improvements in cognitive performance. Accordingly, hippocampal BDNF levels also remained unaltered after GEBR-7b administration, yet were also not affected by genotype. Of not, controversy about BDNF levels in the APPswe/PS1 $1 \mathrm{E} 9$ mouse model does exist, with some studies reporting no 
changes compared to WT level [642, 678], while others report significant decreases in BDNF level at 8 months of age [641]. The BDNF receptors, TrkB, TrkB.T1 and $p 75^{\mathrm{NTR}}$, also were unaffected by genotype or chronic treatment. These results suggest that the relation between CREB-mediated transcription and BDNF protein synthesis is more complex than initially anticipated, and may be differentially regulated in WT and APPswe/PS1 $\mathrm{E} 9$ mice. Indeed, other factors are crucially involved in mediating pCREB-dependent transcription of BDNF. For example, the transcriptional coactivator CREB-binding protein (CBP) can facilitate transcription by activating CREB acetylation [689], and PS1 has shown to regulate CBP levels in vitro as well as in vivo [688, 690-692]. Moreover, alternative DNA methylation of the Bdnf gene [693] can also lead to a mismatch in pCREB activity and BDNF protein expression, and alterations in DNA methylation have been observed within this mouse model (Chouliaras et al., unpublished data).

As PDE4 inhibition can enhance LTP and promote dendritic growth [61, 646], alterations in PSD95 were assessed after chronic GEBR-7b treatment, as a marker for postsynaptic plasticity. The current study could not establish any structural alterations in postsynaptic densities as a result of genotype or chronic PDE4D inhibition. Hippocampal subregionspecific upregulation of both synaptophysin-immunoreactive boutons and postsynaptic densities (the latter being measured with electron microscopy) have been reported previously for this mouse model from 8 months of age [647, 678,694], although decreases in PSD95 compared to WT control have been observed as well for this mouse model, starting as early as 6 months of age $[695,696]$.

\subsection{GEBR-7b treatment does not alter tau signaling or $A \beta$ levels}

Activation of PKA by PDE4 inhibition can lead to a phosphorylation of GSK3 $\beta$, either at threonine 216 , leading to activation of GSK3 3 , or at serine 9, thereby inhibiting its activity. Whereas chronic PDE5 inhibition with sildenafil was able to restore the decrease of pGSK3 $\beta$-Ser9 observed in aged Tg2576 mice, our results did not show any differences in levels of GSK3 $\beta$ or pGSK3 $\beta$-Ser9 in APPswe/PS1 $1 \mathrm{E} 9$ mice compared to WT mice, nor as a result of GEBR-7b treatment. Apparently, decreases in levels of pGSK3 $\beta$-Ser9 are agerelated, as differences in the level of this protein between APPswe/PS1 $\triangle \mathrm{E} 9$ mice and WT mice could not be established at 12 months of age [697], but were present at 16 months of age [698]. To our surprise, 8-month-old APPswe/PS1 1 E9 mice displayed higher levels of total tau compared to WT mice, perhaps indicative of alternative total tau expression in this AD mouse model. Yet, others did not report such findings [697] and the exact regulation of total tau in this mouse model remains to be elucidated. No differences between APPswe/PS1 $1 \mathrm{E} 9$ and WT mice were observed in pTau-Ser396 levels, which confirms previous observations in this mouse model at 12 months of age [697].

Three weeks of GEBR-7b administration did not alter the concentration of hippocampal $A \beta 40$ and $A \beta 42$ in APPswe/PS1 $\triangle E 9$ mice. This indicates that the improvements in long-term spatial memory occurred independently of $A \beta$ production. Comparable results have been 
reported as both in vitro and in vivo administration of GEBR-7b can enhance cAMP levels, without altering $A \beta 40$ and $A \beta 42$ secretion in mouse Neuro-2a cells stably expressing WT human APP695 [523]. Similarly, rolipram treatment could elevate cAMP levels and improve spatial memory performance in AD mice, without affecting $A \beta$ production or deposition [61, 66]. Thus, it appears that PDE4 inhibition can circumvent the detrimental effect of $A \beta$ on synaptic transmission by augmenting intracellular signaling and, thereby, improving memory function.

\subsection{Considerations and concluding remarks}

Although it has been demonstrated that GEBR7b can be beneficial for memory performance when administered acutely in healthy rodents [523] and when administered chronically in a prophylactic manner in $A D$ mice (the current study), the underlying mechanisms explaining GEBR-7b's cognition-enhancing potential remain to be elucidated. Intrahippocampal injection of GEBR-7b has shown to locally upregulate cAMP [523], yet the current study could not establish long-term alterations in downstream pCREB levels. This discrepancy could perhaps be explained by the long time-window between GEBR-7b administration and pCREB read-out in the current study, although others, using a similar paradigm, were able to report structural alterations in hippocampal pCREB levels after PDE4 inhibition [66]. Moreover, as PDE-Is have a vasodilatory function, a central role for enhanced bloodflow or nutrient transport underlying the cognition-enhancing effects has not been unequivocally refuted [657], and further research will need to clarify this issue. In conclusion, it can be said that chronic GEBR-7b treatment can improve memory in an AD mouse model, even after onset of pathology, without altering emotional or neuroendocrine regulation. Altogether, PDE4D inhibition could be considered a novel therapeutic option in the treatment of $A D$ and related dementias.

\section{Acknowledgements}

This study has been funded in part by the Internationale Stichting Alzheimer Onderzoek (ISAO) grant numbers 07551 and 11532 to D.L.A. van den Hove. 


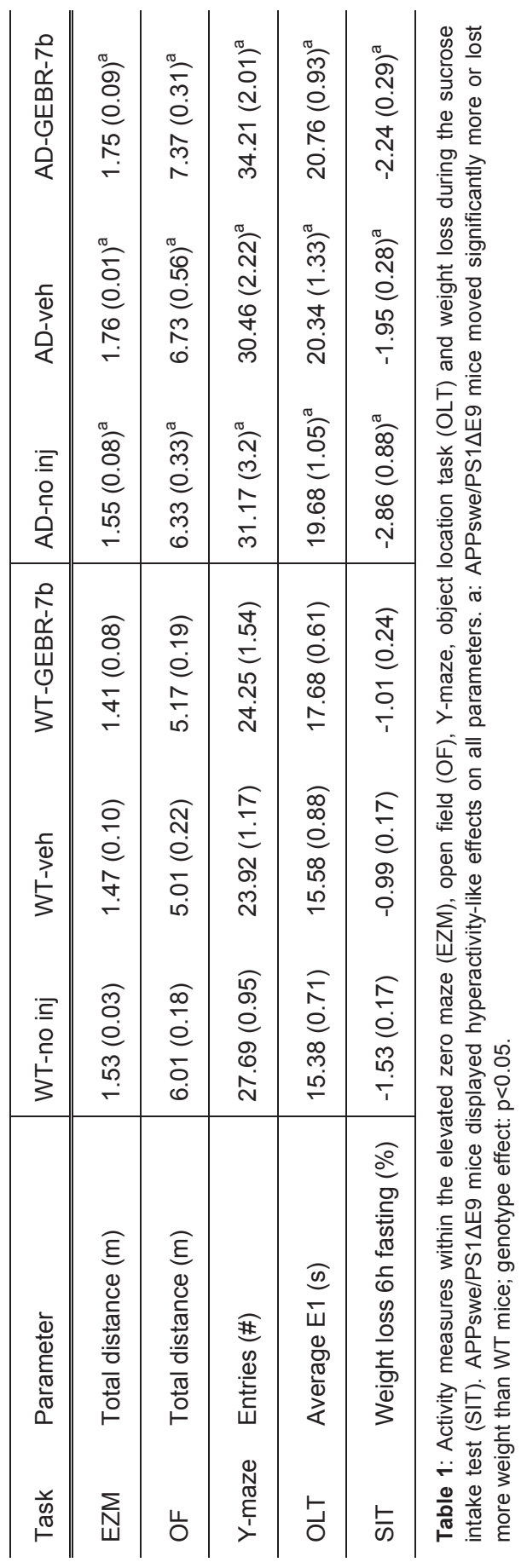




\section{Chapter 8}

Increased mortality in APPswe/PS1 $\triangle \mathrm{E} 9$ mice after chronic fluoxetine treatment: a role for epileptic seizures?

Annerieke SR Sierksma, Jos Prickaerts, Govert Hoogland, Tim Vanmierlo, Fred W van Leeuwen, Harry WM Steinbusch, Daniel LA van den Hove 


\section{Abstract}

Epidemiological research suggests that major depression (MD) is a risk factor for the development of Alzheimer's disease (AD). Conversely, a history of antidepressant drug use decreases the risk for developing $A D$ and is beneficial for cognitive function in both $M D$ and $A D$ patients. To investigate how antidepressants affect the development of $A D, 5$-month-old APPswe/PS1 $\triangle E 9$ mice were treated with the selective serotonin reuptake inhibitor (SSRI) fluoxetine $(15 \mathrm{mg} / \mathrm{kg})$. We surprisingly observed that several mice displayed seizure behavior, including bilateral forelimb clonus, head and forelimb extensions, wild running/jumping/bouncing, rearing and falling as well as generalized tonic-clonic seizures. Starting from day 4 of treatment, the number of mice displaying this behavior increased to a total of $45 \%$ of the animals at day 17 , after which it was decided to cease treatment. Concomitantly with the vast increase in seizures, unusually high mortality rates were observed within the fluoxetine-treated APPswe/PS1 $\triangle E 9$ group. Binary logistic regression analysis revealed that having the APPswe/PS1 $1 \mathrm{E} 9$ genotype and fluoxetine treatment were significant predictors for seizure development. Moreover, seizure development, the APPswe/PS1 $\triangle E 9$ genotype and fluoxetine treatment were significant predictors for premature death. Although spontaneous behavioral seizures and EEG-recorded spontaneous epileptic discharges have been observed before in this mouse model, the finding that this behavior can be triggered by an antidepressant is new. We postulate that the APPswe/PS1 $1 \mathrm{E} 9$ mouse model may be characterized by altered drug metabolism, which, together with the non-linear pharmacokinetics of fluoxetine, may result in sustainably high, and possibly accumulating, plasma (nor)fluoxetine levels. Fluoxetine has proconvulsive properties, yet is also regularly prescribed to $A D$ patients, who are already at increased risk for developing epilepsy. In our view, until the mechanisms of fluoxetine-induced seizures are further elucidated, its use in AD patients should be monitored carefully. 


\section{Introduction}

Alzheimer's disease (AD) is the most common cause of dementia and is characterized by progressive cognitive impairment, including memory loss. $A D$ is diagnosed at post mortem brain examination by the presence of two types of protein aggregates: extracellular amyloid$\beta(A \beta)$ plaques and intracellular neurofibrillary tau tangles. Its etiopathogenesis is currently still unknown, although several autosomal dominant mutations in the amyloid precursor protein (APP) and presenilin 1 (PS1) and 2 (PS2) genes have been described, accounting for the familial $A D(F A D)$ cases [699]. Among the sporadic cases, several genetic risk factors have been identified, including apolipoprotein 4 (APOE4) and clusterin, while in addition, a wide variety of environmental exposures have been proposed to heighten or lessen the risk of developing AD (for an overview, see [504]).

Among these environmental risk factors are chronic stress and stress-related disorders, such as major depression (MD), which elevate the chance of AD development. Indeed, meta-analysis has indicated that having a history of depression increased the likelihood of $A D$ by twofold [460], and can accelerate the timing of disease onset in the FAD cases [75]. Moreover, a lifetime history of MD leads to higher expression of hippocampal plaques and tangles and a more rapid cognitive decline when compared to AD patients without a history of MD [128]. Similarly, in mouse models of AD, chronic stress exposure has been shown to lead to an accelerated manifestation of cognitive deficits with concomitant increases in $A \beta$ and tau pathology and signs of neurodegeneration [76, 134, 505].

Conversely, repeated use of antidepressant medication can decrease the risk for $A D$ development [700] and slow down cognitive decline [701, 702]. Likewise, in vivo studies have demonstrated the efficacy of antidepressant treatment on cognitive performance in mouse models of $A D$ [78, 495, 703]. In fact, the beneficial effects of selective serotonin reuptake inhibitors (SSRIs), a class of antidepressants, are probably exerted via reductions in $A \beta$ production [495, 496, 703-705]. The initial aim of the current study was therefore to investigate whether chronic fluoxetine treatment in APPswe/PS1 $\Delta \mathrm{E} 9$ mice could alter expression of AD-related symptoms and neuropathology, possibly induced by chronic injection stress, and to elucidate via which mechanisms these effects would be achieved.

\section{Material \& Methods}

\subsection{Animals}

All experimental animal research was executed according to protocols approved by the local Animal Ethical Committee of Maastricht University, Maastricht, the Netherlands (UM-DEC 2009-177) and met governmental guidelines. All animals were housed under a $12 \mathrm{~h}$ light/dark cycle (lights on at 7:00 A.M, unless specified otherwise) in standard Makrolon cages on sawdust bedding, placed in an individually ventilated cage (IVC) unit, regulating temperature 
(approximately $20^{\circ} \mathrm{C}$ ) and humidity (between $40-60 \%$ ). A radio, which was playing softly, provided background noise in all rooms. Food and water was provided ad libitum throughout the study. Male C57BL/6 wild-type (WT) and APPswe/PS1 $\Delta$ E9 mice (on C57BL/6 background) were obtained as described in [706] and were the littermates of the mice described in that study. At approximately 3.5 months of age, mice were housed individually to avoid aggressive behavior among the APPswe/PS1 $\triangle \mathrm{E} 9$ mice. Weight of the offspring was monitored regularly to have a general indication of well-being.

\subsection{Drug treatment}

At 19 weeks of age, fluoxetine treatment was commenced for APPswe/PS1 $1 \mathrm{E} 9$ and WT mice by administering a daily per os (p.o.) injection $(5 \mathrm{ml} / \mathrm{kg})$ of vehicle solution $(0.5 \%$ methyl 2-hydroxyethyl cellulose and $0.005 \%$ dimethylsulphoxide) or fluoxetine $\mathrm{HCl}^{-}(15 \mathrm{mg} / \mathrm{kg})$ dissolved in vehicle. Fluoxetine $\mathrm{HCl}^{-}$was kindly provided by Solvay Pharmaceuticals (Weesp, The Netherlands). Solutions were prepared freshly each day ( $\mathrm{pH} \approx 6.0$ ). To assess any effects of the 3 week injection paradigm, a 'no-injection' group was added. To reduce the number of animals, this study was done in parallel to the study described in Sierksma et al. [706]. Therefore, the no-injection group described in both studies is comprised of the same animals. In total, 6 experimental groups were formed: WT animals not receiving any injections (WT-no inj, $n=12$ ), WT animals receiving vehicle injections (WT-veh, $n=12$ ), WT animals receiving fluoxetine (WT-fluox, $n=12$ ), APPswe/PS1 $\triangle E 9$ mice not receiving any injections (AD-no inj, $n=12$ ), APPswe/PS1 $\triangle E 9$ mice receiving vehicle injections (AD-veh, $\mathrm{n}=13$ ), and APPswe/PS1 $\triangle E 9$ mice receiving fluoxetine (AD-fluox, $n=13$ ). To dissociate between stress from frequent handling and injection stress, the no-injection group was also handled, but not restrained or injected.

\subsection{Behavioral assessment}

For a global timeline of the behavioral tests conducted in this experiment, see Figure 1. Experimental groups without daily injections were included to control for possible adverse effects of chronic injections stress $[666,667]$. One day after the last injection, the mice were subjected to the sucrose intake test (SIT) and the elevated zero maze (EZM), to assess the effects on affective behavior. Afterwards, mice were subjected to the open field (OF) test to assess locomotor behavior and to the $\mathrm{Y}$-maze spontaneous alternation test ( $\mathrm{Y}$-maze) to measure working memory function. Three weeks later, at 26 weeks of age, mice were habituated for the object location task (OLT) and subsequently tested to measure long-term spatial memory. Following the OLT, the forced swim test (FST) was conducted as a measure of depressive-like behavior. 


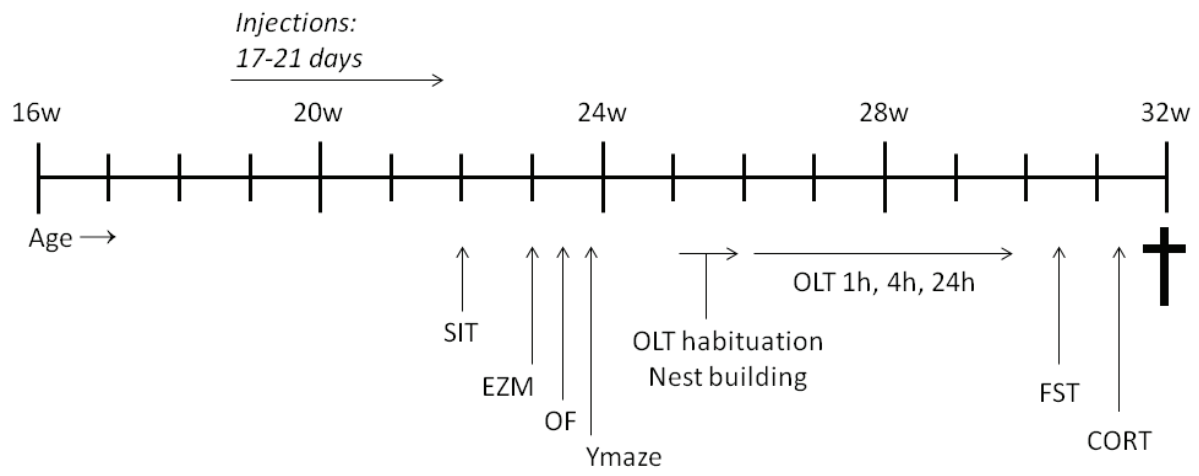

Figure 1: Experimental design. Fluoxetine (15 mg/kg bodyweight) or vehicle was injected per os, once daily to wild-type (WT, $n=12 /$ treatment) or APPswe/PS1 $\triangle E 9$ ( $n=13 /$ treatment) mice. A no-injection group was added to control for effects of injection stress ( $n=12 /$ genotype). Because $45 \%$ of fluoxetine-treated APPswe/PS1 $1 \mathrm{E} 9$ mice displayed signs of seizures after 17 days of treatment, it was decided to discontinue treatment in this group, whereas treatment was continued for all other experimental groups until day 21. Following treatment, mice were subjected to behavioral testing for locomotion, anxiety, depressive-like behavior and cognitive performance. Finally, blood samples were drawn from the vena saphena followed by corticosterone assessment, and death at 8 month of age. W: weeks of age; SIT: sucrose intake test; EZM: elevated zero maze; OF: open field; OLT: object location task; FST: forced swim test; CORT: blood withdrawal for corticosterone determination.

\subsection{1. $S I T$}

The SIT is often used to measure anhedonia, one of the core symptoms of depressive behavior $[668,669]$. Mice were first habituated to the $1 \%$ sucrose solution, by replacing their drinking water with the sucrose solution for 24 hours. On the day of the SIT, mice were deprived of food and water for 6 hours (starting at 7 AM), after which they received a bottle of $1 \%$ sucrose solution for 1 hour. Sucrose intake was recorded by weighing the bottles before and after the intake and corrected for body weight.

\subsection{2. $E Z M$}

The EZM is an effective tool to measure anxiety in a rodent [527]. The setup consisted of a black plastic circular runway (diameter $46 \mathrm{~cm}$, width $5.5 \mathrm{~cm}$ ) that was transparent for infrared light and elevated $20 \mathrm{~cm}$ above the floor [670]. The arena was divided into 4 quadrants: 2 opposing open quadrants with a border (height $3 \mathrm{~mm}$ ) to prevent the mouse from stepping down, and 2 opposing closed quadrants with side walls (height $20 \mathrm{~cm}$ ). Each mouse was placed individually into one of the open arms close to and facing a closed arm, and was allowed to explore the arena for 5 min under almost dark conditions. After each session the maze was thoroughly cleaned using a $70 \%$ ethanol solution. The movements of each mouse were tracked automatically using an infrared camera and Ethovision Pro (Noldus, The Netherlands) $[528,590]$. Parameters for anxious behavior were the total distance traveled 
and percentage of time spent in the closed arms, corrected for possible freezing behavior at the start of the experiment (i.e. the latency to enter one of the closed arm).

\subsection{3. $O F$}

The open field arena is used to assess locomotor behavior [671]. The apparatus was made of acrylic and consisted of a grey square arena $(50 \times 50 \times 25 \mathrm{~cm})$, subdivided by grey walls (height: $25 \mathrm{~cm}$ ) into 4 separate arenas of $25 \times 25 \times 25 \mathrm{~cm}$, leaving the top open. One mouse was introduced per arena, thereby testing 4 mice simultaneously. After each session the arena was thoroughly cleaned using a $70 \%$ ethanol solution. The mouse was placed in the centre of the arena, being able to move around freely for $20 \mathrm{~min}$. The movements of each mouse were tracked automatically by using a video camera and Ethovision Pro (Noldus, The Netherlands). The total distance moved within 20 minutes was used as a measure of locomotor behavior.

\subsubsection{Y-maze}

Spatial working memory was assessed by using the Y-maze spontaneous alternation test, as previously described [524]. The symmetrical grey $\mathrm{Y}$-maze made of acrylic consisted of 3 arms, with all arms being apart from the others at a $120^{\circ}$ angle. Each arm was $40 \mathrm{~cm}$ long, $17 \mathrm{~cm}$ high, $4 \mathrm{~cm}$ wide at the bottom and $13 \mathrm{~cm}$ wide at the top. Each mouse was placed in the centre of the $Y$-maze and was free to explore the arena for $6 \mathrm{~min}$. The number of entries was counted per mouse: an entry required that both hind paws of the animal had to be placed completely inside the arm. A mouse would be making a triad when it visited all 3 arms consecutively. As a measure for working memory, the percentage of alternations that the mouse made was calculated, being the number of triads divided by the maximum possible alternations (i.e. the total number of entries minus 2$) \times 100$. If a mouse scored significantly above $50 \%$ alternations (the chance level for choosing the unfamiliar arm), this was indicative of functional working memory. Between sessions, the maze was cleaned thoroughly with a $70 \%$ ethanol solution.

\subsubsection{OLT}

Long-term spatial memory was tested using the OLT, as described previously [522]. In brief, the setup consisted of a circular arena, $43 \mathrm{~cm}$ in diameter. The $40 \mathrm{~cm}$ high wall was divided into two halves, one made of white, the other made of transparent polyvinyl chloride. Two objects were placed symmetrically in the middle of the arena. Four different sets of objects were used, and each object was available in triplicate. The different objects were: 1) a massive metal cube $(2.5 \times 5 \times 7.5 \mathrm{~cm})$ with 2 holes (diameter $1.5 \mathrm{~cm}), 2)$ a white plastic cube with rounded off edges placed on a small stand of the same material (maximal diameter 3.5 $\mathrm{cm}$ and total height $11.5 \mathrm{~cm}$ ), 3) a brass cylinder with a tapering top (maximal diameter $3 \mathrm{~cm}$ and total height $11.5 \mathrm{~cm})$ and 4) a massive aluminum cube with a tapering top $(4.5 \times 4.5 \mathrm{x}$ $8.5 \mathrm{~cm}$ ). Mice could not displace the objects. The task was conducted as previously 
described [522, 523]. To habituate the animals to behavioral testing, all mice were first handled, weighed and were able to explore the empty arena of the OLT for 4 minutes. Subsequently, the animals were habituated to the $1 \mathrm{~h}$ and $4 \mathrm{~h}$ interval of the OLT. A testing session consisted of 2 trials, with each trial lasting 4 minutes. During the first trial (T1) the arena contained 2 identical objects at fixed locations. A mouse was introduced into the arena by placing it with its nose to the middle of the transparent segment of the wall. After the first exploration period had elapsed, the mouse was placed back in its home cage. Subsequently, after a predetermined time interval (e.g. $1 \mathrm{~h}$ or $4 \mathrm{~h}$ ) the mouse was returned to the arena for the second trial (T2). For T2, one object was relocated, which was randomized in a balanced manner over the groups and the different sessions (both location and object) to reduce potential bias due to preference for particular objects. The time spent exploring the objects during T1 and T2 was recorded manually using a computer. Exploring behavior was defined as follows: directing its nose to the object at a distance of no more than $1 \mathrm{~cm}$ and/or touching the object with its nose. Sitting on the object was not considered exploration. In order to avoid the presence of olfactory cues, all objects and the arena were cleaned thoroughly with ethanol between sessions. In order to determine whether a mouse had remembered the familiar location of the object, several measures were used. The amount of exploration time is recorded in T1 (named e1) and T2 (e2). The discrimination index d2 calculates whether the mouse spends more time at the novel location while correcting for the total e2: (exploration time new location - exploration time old location)/e2. The latter will be an indication of recognition of the novel location of the objects, i.e. spatial memory. A minimum of 1.5 seconds exploration was set as an inclusion criterion to reliable measure memory performance [590].

\subsubsection{Nesting behavior}

Nesting behavior is dependent on hippocampal functioning [672, 673], and is part of the normal cage behavior in a mouse (activities of daily life [674]). Gross differences in nestbuilding were assessed as described previously [675]. In short, the mice were provided with equal pieces of paper towel. After $24 \mathrm{~h}$, the quality of the nest was assessed, scoring 0 when no nest was produced and 1 if a nest was constructed. A nest was defined as follows: the tissue was shredded and manipulated into a nest to provide the mouse with warmth and shelter.

\subsubsection{FST}

The FST is a widely used behavioral task to study depressive-like behavior in rodents [529]. Each mouse was individually placed into a perspex cylinder (diameter $17 \mathrm{~cm}$, height $40 \mathrm{~cm}$ ) filled with $30^{\circ} \mathrm{C}\left(+/-2^{\circ} \mathrm{C}\right)$ water to a height of $15 \mathrm{~cm}$ for $6 \mathrm{~min}$. Each session was scored automatically with Ethovision Pro (Noldus, The Netherlands) [590]. The total distance swum was used as a measure of depressive-like behavior, i.e. a lesser distance swum being indicative of heightened despair [510]. 


\subsection{Blood sampling and corticosterone radioimmunoassay}

In order to assess hypothalamo-pituitary-adrenal (HPA) axis responsiveness to a stressor, plasma corticosterone was measured at baseline, during stress and after recovery. First, the mice were moved to another room and taken out of their home cage, immediately after which a $50 \mu \mathrm{l}$ blood sample was drawn from the vena saphena (basal corticosterone concentration) using heparinised blood collection tubes (Microvette ${ }^{\circledR}$ CB300, Sarstedt, Germany). Subsequently, the mouse was restrained for 20 minutes in a glass cylinder to induce a stress response, followed by a second $50 \mu \mathrm{l}$ blood sample collection (stressinduced corticosterone concentration). Finally, animals were returned to their home cage and housing room and allowed to recover for $60 \mathrm{~min}$ after which a third $50 \mu \mathrm{l}$ sample was taken ('recovery' corticosterone concentration). All blood samples were kept on ice and subsequently centrifuged at $3000 \mathrm{rpm}$ for $10 \mathrm{~min}$ at $4^{\circ} \mathrm{C}$, after which plasma was isolated, frozen down to $-80^{\circ} \mathrm{C}$ and stored until further processing. For the corticosterone radioimmunoassay, an ImmuChem ${ }^{\mathrm{TM}}$ Double Antibody Corticosterone ${ }^{125}$ I RIA Kit for rodents (MP Biomedicals, Orangeburg, NY, USA) was used. The assay was performed according to the manufacturer's instructions. Briefly, for each sample, $5 \mu \mathrm{l}$ serum was diluted in steroid diluents (1:100 for basal and recovery samples and 1:200 for stress samples). Per $50 \mu \mathrm{l}$ of diluted sample, $50 \mu \mathrm{l}$ of corticosterone ${ }^{-125} \mathrm{I}$ and $100 \mu \mathrm{l}$ anti-corticosterone was added, and samples were incubated at room temperature (RT) for $2 \mathrm{~h}$. Afterwards, $250 \mu \mathrm{l}$ precipitant solution was added and tubes were centrifuged at $2300 \mathrm{rpm}$ for $15 \mathrm{~min}$. Supernatant was aspirated and the precipitate was counted in a Wizard Gamma Counter 2470 (Perkin Elmer, Waltham, MA, USA).

\subsection{Statistical analysis}

All data are presented as mean and standard error of the mean (SEM). Two-way analysis of variance (ANOVA) was performed for comparison between groups accounting for the main and interactive effects of genotype (WT, APPswe/PS1 $1 \mathrm{E} 9$ ) and treatment (no injection, vehicle, fluoxetine). Repeated measures ANOVA were performed to assess OLT D2 scores over the different intervals, as well as for the corticosterone secretion (LN-transformed) over time. As indications for memory performance, a one-sample t-test was performed for the $Y$ maze spontaneous alternation test and the OLT, with testing values 50 and 0 , respectively. Statistical assessment of nest building behavior was performed with the Pearson $x^{2}$ test. Binary logistic regression was performed on the dichotomous dependent variables premature death $(0=$ no, $1=y e s)$ and the observed epileptic convulsions $(0=$ no, $1=$ yes) (see section 3.1.), with genotype ( $0=\mathrm{WT}, 1=\mathrm{APPswe} / \mathrm{PS} 1 \Delta \mathrm{E} 9)$, fluoxetine treatment $(0=\mathrm{no}$, $1=y e s)$, having received injections ( $0=$ no, $1=$ yes) and observed epileptic convulsions $(0=$ no, $1=y e s$, when applicable) as independent variables. Statistical significance was set an a level of 0.05 and all tests were two-tailed. All statistical analyses were conducted using the Statistical Package for the Social Sciences (SPSS 15.0, SPSS Inc., Chicago, IL, USA). All 
graphs were constructed in GraphPad Prism (version 4, GraphPad Software, San Diego, CA, USA).

\section{Results}

\subsection{Unexpected premature death}

Originally, we had planned a treatment period of 21 days consecutively. However, at day 4 , one of the fluoxetine-treated AD mice displayed seizure behavior, i.e. oral and facial automatisms and bilateral forelimb clonus, while being restrained. From that day onwards, an increasing number of mice displayed seizure behavior, including bilateral forelimb clonus, head and forelimb extensions, wild running/jumping/bouncing, rearing and falling, and/or generalized tonic-clonic seizures. This often occurred right before injection (while being fixated), or immediately following injections. In other cases, the observer would hear noise from the cages reminiscent of 2 animals fighting. Upon inspection, it became clear that one mouse was bouncing through the cage, followed by a generalized tonic-clonic seizure while lying on its side. The percentage of mice displaying seizure behavior is depicted in Figure $2 \mathrm{~A}$. Yet, these data are based on daily routine inspection of the cages, and may thus underestimate the actual number of mice showing seizure behavior.

Following these initial seizure observations, the number of premature deaths increased rapidly (see Figure 2B). For ethical reasons, it was decided to discontinue fluoxetine treatment of APPswe/PS1 $1 \mathrm{E} 9$ mice after 17 days. Performing binary logistic regression analysis revealed that having epileptic seizures could be significantly predicted by the APPswe/PS1 $\triangle E 9$ genotype $(\mathrm{OR}=15.40,95 \% \mathrm{Cl}=1.79-132.85, \mathrm{p}<0.05)$ and fluoxetine treatment $(\mathrm{OR}=7.09,95 \% \mathrm{Cl}=1.79-28.09, \mathrm{p}<0.01)$, but not by injections per se $(\mathrm{OR}<0.00$; see table 1). Moreover, binary logistic regression analysis indicated that premature death could be significantly predicted by having the APPswe/PS1 $\triangle E 9$ genotype (OR=11.17, 95\% confidence interval $(C l)=1.03-120.59, p<0.05)$, observed epileptic seizures $(O R=18.97$, $95 \% \mathrm{Cl}=3.37-106.89, \mathrm{p}<0.01)$, and fluoxetine treatment $(\mathrm{OR}=8.47,95 \% \mathrm{Cl}=1.50-47.92$, $\mathrm{p}<0.05)$. Receiving injections per se did not predict the likelihood of dying prematurely (OR=0.56, 95\% $\mathrm{Cl}=0.05-6.51$, n.s.; see table 2 ).

\subsection{Weight}

Weight of the offspring was monitored regularly throughout the experiment, serving as an indication of general wellbeing (see Figure $2 \mathrm{C}$ ). Body weight was not different between the groups before the start of injections, i.e. at 18 weeks of age. Repeated measures ANOVA demonstrated a significant effect of time $(F(2.03,103.57)=17.01, p<0.001)$ on body weight, indicating that weight changed over time. The test of between-subject effects indicated that overall significant effects were found for treatment $(F(1,51)=4.61, p<0.05)$ and 
genotype*treatment $(F(1,51)=3.64, p<0.05)$ on body weight. LSD post hoc analysis revealed that weights of the AD-fluox group were significant lower than the AD-no inj $(p<0.01)$ and the $A D$-veh group $(p<0.01)$, while no significant differences were found between the WT groups (see figure $1 \mathrm{C}$ ). Weight data of week 21, recorded the day after cessation of treatment, demonstrated a significant genotype treatment effect $(F(1,70)=3.17, p<0.05)$. LSD post hoc analysis revealed that the AD-fluox mice were significantly lighter than AD-veh mice, possibly reflecting a decrement in overall well-being (see Figure $2 \mathrm{C}$ ).

\begin{tabular}{l|lllll}
\hline Included & $\beta(\mathrm{SE})$ & Wald & $\mathrm{df}$ & $\mathrm{OR}$ & $95 \% \mathrm{Cl}$ \\
\hline \hline APPswe/PS1 $\triangle \mathrm{E9}$ genotype & $2.73(1.10)$ & 6.19 & 1 & $15.40^{*}$ & $1.79-132.85$ \\
Fluoxetine & $1.96(0.70)$ & 7.78 & 1 & $7.09^{* *}$ & $1.79-28.09$ \\
Injections & $18.42(7652.45)$ & 0.00 & 1 & $<0.00$ & $0.00-\mathrm{nc}$ \\
Constant & $-23.17(7652.45)$ & 0.00 & 1 & 0.00 & \\
\hline
\end{tabular}

Table 1: Results of the binary logistic regression model on seizures. Seizures were significantly predicted by the APPswe/PS1 $1 \mathrm{E} 9$ genotype and fluoxetine treatment, but not by receiving injections. R2 (Hosmer\&Lemeshow) $=.24$, Model X2 $(3)=23.28(p<0.001)$. SE: standard error; Wald: Wald statistic with a chi-square distribution; df: degrees of freedom; OR: odds ratio; $\mathrm{Cl}$ : confidence interval; nc: not calculated. ${ }^{* *}: p<0.01 ;{ }^{*}: p<0.05$.

\begin{tabular}{l|lllll}
\hline Included & $\beta(\mathrm{SE})$ & Wald & $\mathrm{df}$ & OR & $95 \% \mathrm{Cl}$ \\
\hline \hline APPswe/PS1 $\triangle$ E9 genotype & $2.41(1.21)$ & 3.95 & 1 & $11.17^{*}$ & $1.03-120.59$ \\
Observed seizures & $2.94(0.88)$ & 11.13 & 1 & $18.97^{* *}$ & $3.37-106.89$ \\
Fluoxetine & $2.14(0.88)$ & 5.83 & 1 & $8.47^{*}$ & $1.50-47.92$ \\
Injections & $-0.59(1.26)$ & 0.22 & 1 & 0.56 & $0.05-6.51$ \\
Constant & $-4.91(1.51)$ & 10.55 & 1 & $0.01^{*}$ & \\
\hline
\end{tabular}

Table 2: Results of the binary logistic regression model on premature death. Premature death was significantly predicted by APPswe/PS1 $\triangle \mathrm{E} 9$ genotype, observed seizures and fluoxetine, but not by receiving injections. R2 (Hosmer\&Lemeshow) =.39, Model X2 (4)=37.30 $(p<0.001)$. SE: standard error; Wald: Wald statistic with a chi-square distribution; df: degrees of freedom; OR: odds ratio; Cl: confidence interval. ${ }^{* *}$ : $p<0.01 ;{ }^{*}: p<0.05$. 


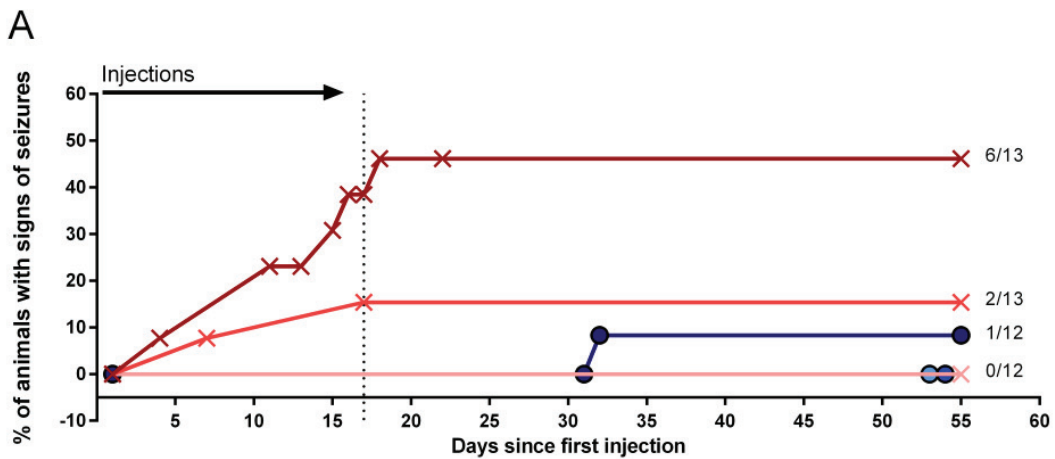

B

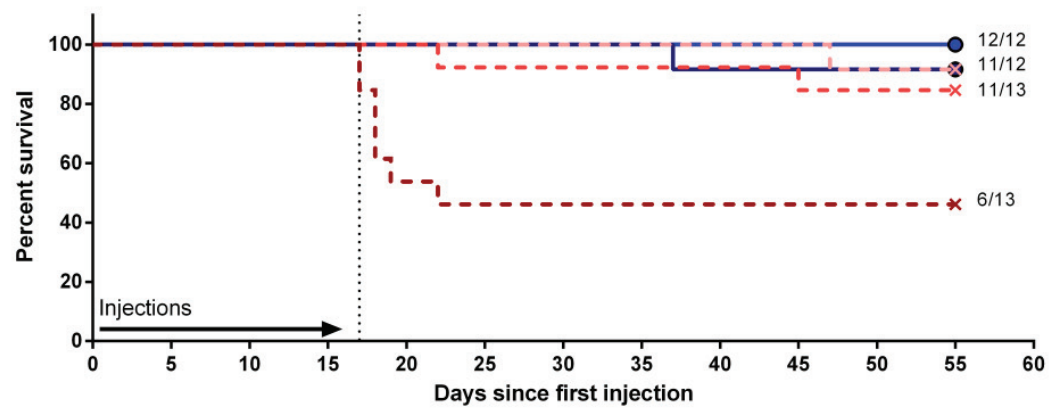

C

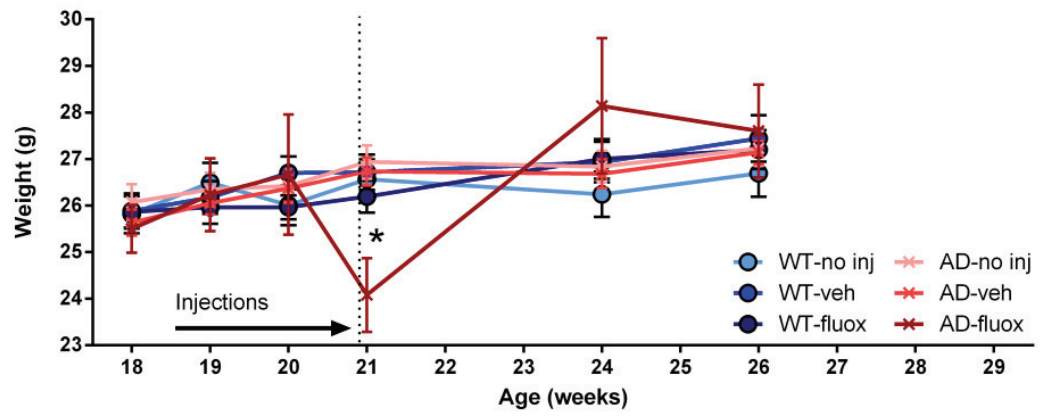

Figure 2: Cumulative incidence of mice displaying seizures (A), survival rate $(B)$ and body weight changes $(C)$ over time. A: Due to an increasing number of animals that displayed seizures, fluoxetine treatment of APPswe/PS1 $1 \mathrm{E} 9$ mice was discontinued after 17 days (indicated by a vertical dashed line). At the end of each line the number of animals with seizures/total number of animals is indicated. B: Six APPswe/PS1 $\triangle \mathrm{E} 9$ mice died within 1 week after cessation of fluoxetine treatment. At the end of each line the number of living animals at day $55 /$ total number of animals is indicated. C: The AD-fluox group demonstrated a striking weight drop during the second week of injections, indicative of poor well being. Surviving AD-fluox mice did however continue to develop normally. *: $p<0.05$, AD-Fluox compared to AD-no inj and AD-veh. 


\subsection{Behavioral assessment}

\subsubsection{SIT, EZM, FST and OF}

Results of the SIT and EZM indicated that there are no significant differences between the groups in terms of anhedonia and anxiety levels, respectively. The FST data revealed a significant genotype*treatment effect $(F(1,60)=3.59, p<0.05)$. LSD post hoc analysis showed that WT-fluox mice swim a significantly shorter distance than WT-no inj mice, indicative of increased depressive-like behavior (see Figure 3). No differences were observed in terms of locomotor behavior, as measured by the OF test (data not shown).

\subsubsection{Nest building behavior and Y-maze spontaneous alternation test}

Nest building behavior and spatial working memory performance was not significantly different between the groups (see Figure 4).

\subsection{3. $O L T$}

Repeated measures ANOVA on all 3 intervals demonstrated a significant interval effect $(F(1.76,100.06)=3.96, p<0.05)$, indicating that spatial memory performance changes over the intervals. Overall, however, a significant genotype effect was found $(F(1,57)=4.77$, $\mathrm{p}<0.05$ ), with APPswe/PS1 $\mathrm{E} 9$ mice performing significantly worse in the OLT than WT mice (see Figure 4). Nonetheless, at the different intervals, no significant difference in performance could be observed between the groups (data not shown).

\subsection{Corticosterone secretion}

Repeated measures ANOVA revealed a significant condition effect $(F(1.50,79.59)=55.50$, $\mathrm{p}<0.001)$ on plasma corticosterone concentrations, indicating that corticosterone concentrations differ across stress conditions. However, the groups did not differ in their HPA axis response after a stressor (see Figure 3D). 
A

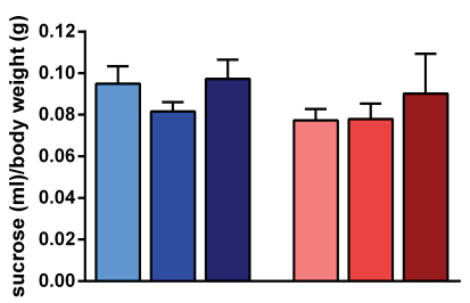

C

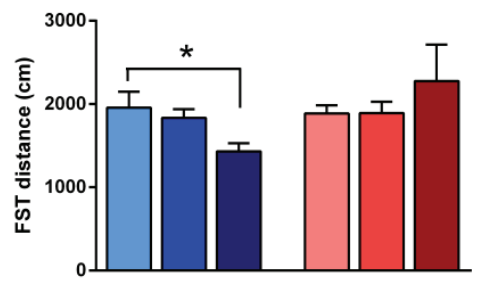

B

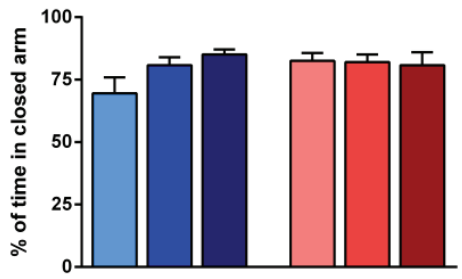

$\mathrm{D}$

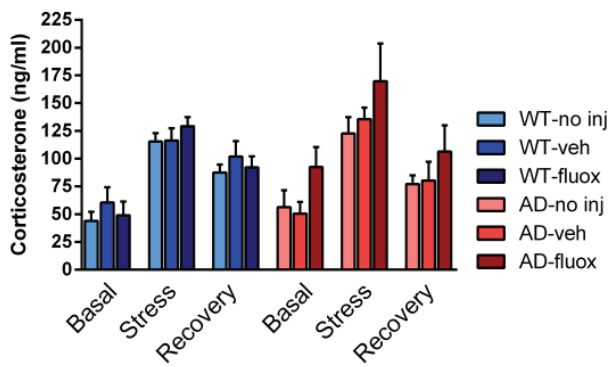

Figure 3: Results of the sucrose intake test (SIT; A), elevated zero maze (EZM; B), the forced swim test (FST; C) and hypothalamic-pituitary-adrenal (HPA) axis regulation (D). Bars represent mean and SEM per group of untreated wild-type (WT) mice (WT-no inj, $n=12$ ), vehicle-treated WT mice (WT-veh, $n=12$ ), fluoxetine-treated WT mice (WT-fluox, $n=11$ ), untreated APPswe/PS1 $\triangle E 9$ (AD) mice (AD-no inj, $n=11$ ), vehicle-treated $A D$ mice ( $A D$-veh, $n=11$ ), and fluoxetine-treated $A D$ mice ( $A D$-fluox, $n=6$ ). $A$ : Sucrose intake did not differ between the groups, indicating that chronic injections or fluoxetine treatment did not alter levels of anhedonia. B: Levels of anxiety were not different among the groups. C: In the FST, WT mice treated with fluoxetine displayed more depressive-like behavior than the WT-no inj group. D: Corticosterone secretion during basal, stressful and recovery condition were not significantly different between the groups. *: $p<0.05$, LSD post hoc. 

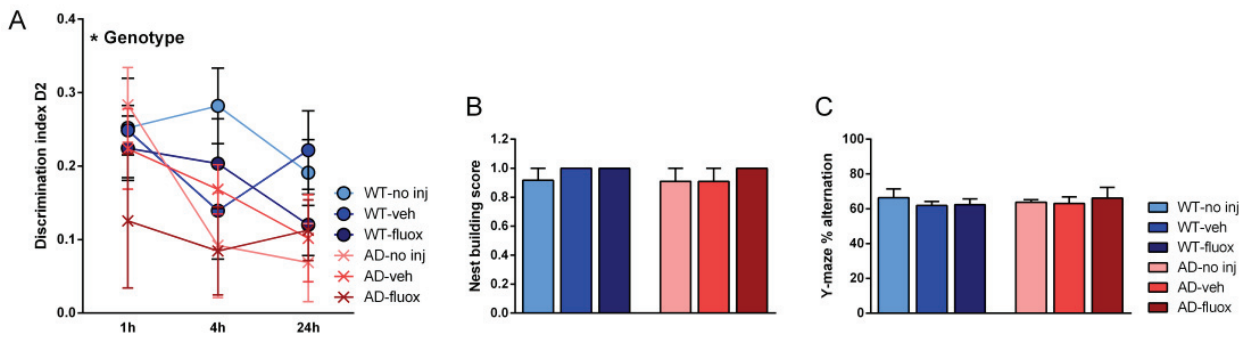

Figure 4: Hippocampus-dependent behavior as measured by the object location task (OLT, A), nest building behavior (B) and the Y-maze spontaneous alternations test (C). Bars represent mean and SEM per group of untreated wild-type (WT) mice (WT-no inj, n=12), vehicle-treated WT mice (WT-veh, $n=12$ ), fluoxetine-treated WT mice (WT-fluox, $n=11$ ), untreated APPswe/PS1 $\triangle E 9$ (AD) mice (AD-no inj, $n=11$ ), vehicle-treated $A D$ mice ( $A D-v e h, n=11$ ), and fluoxetine-treated $A D$ mice (AD-fluox, $n=6$ ). $A$ : Performance in the OLT is expressed as the D2 discrimination index ([exploration novel location exploration familiar location]/total exploration time), with higher scores being indicative of better memory function. Overall, APPswe/PS1 $1 \mathrm{E} 9$ mice had significantly worse long-term spatial memory than WT mice, as is observed by the significant genotype effect. No treatment effect was observed. B: Nest building behavior: score 1 was given when a nest was constructed $24 \mathrm{~h}$ after having received nesting material. No differences were found between the groups. C: Spatial working memory was assessed in the Y-maze, but no differences in alternating behavior were observed between the groups. ${ }^{*}$ Genotype: $p<0.05$.

\section{Discussion}

The aim of the present study was to explore whether a more depressive-like phenotype would accelerate AD-related symptoms and whether these alterations could be augmented by chronic fluoxetine treatment.

\subsection{Chronic oral injections do not induce alterations in affective phenotype}

Our results indicate that no significant differences could be found between the no-injection and the vehicle-treated groups in terms of anxious or depressive-like behavior, as measured with the EZM, SIT and FST. With respect to the FST it was shown that, when compared to the no-injection group, fluoxetine treatment induced a more depressive-like phenotype, specifically in WT mice. Similar results have been reported before in C57BL/6 mice after 16day treatment with fluoxetine, as treated mice spent more time immobile in the FST and also showed more anxiety in the elevated plus maze [707]. Although the increased depressivelike behavior after fluoxetine treatment could be indicative of withdrawal symptoms [708, 709], these effects are least commonly reported for fluoxetine (due to its long half-life) [710], and mostly cease after 3 weeks post-treatment in humans [708]. Chronic p.o. injections also did not alter HPA axis response to a stressor, as the corticosterone concentrations in plasma during basal, stressful and recovery conditions were not different between the groups. In 
rats, repeated injections appear sufficiently stressful as they can induce a depressive-like phenotype and are able to decrease hippocampal BDNF levels [667, 711], yet, we could not corroborate these findings in mice.

\subsection{Fluoxetine-treated APPswe/PS1 1 E9 mice display signs of epilepsy}

To our surprise, we observed that almost half of all fluoxetine-treated APPswe/PS1 $\triangle E 9$ mice in this study developed seizures after 17 days of treatment. This was accompanied by an unusually high mortality rate. Statistical analysis revealed that fluoxetine treatment and having the APPswe/PS1 $\triangle \mathrm{E} 9$ genotype significantly increased the likelihood of developing seizures, thereby increasing the risk of premature death. It has previously been reported that up to $65 \%$ of APPswe/PS1 $1 \mathrm{E} 9$ mice exhibit spontaneous behavioral seizures and that $100 \%$ of APPswe/PS1 $\triangle \mathrm{E} 9$ mice show EEG-recorded spontaneous epileptic discharges [712, 713]. On a cellular level, APPswe/PS1 $\triangle \mathrm{E} 9$ mice further exhibited decreased action potential thresholds, suggestive of neuron hyperexcitability [713]. Mice overexpressing WT or mutant human APP also exhibit tonic-clonic seizures [714], as well as non-convulsive epileptogenic activity in both the cortex and hippocampus [715].

Epidemiological studies have demonstrated that $A D$ patients are at an increased risk for epileptic seizures as well [716-719]. Particularly patients with a young age of dementia onset or during early stages of the disease can be up to 87 times more likely to suffer from seizures [716, 717]. Certain behaviors, such as wandering, disorientation and memory disturbances, can also be caused by forms of epilepsy, and can easily be misinterpreted as $A D$ [720-722]. It has therefore been suggested that non-convulsive epileptiform activity could be responsible for certain cognitive deficits manifested in AD [723]. Associations between epilepsy and $A D$ are particularly prominent in patients with FAD mutations. Interestingly, PS1 mutations have been linked most strongly to vulnerability of epileptic seizures, as $20 \%$ of all PS1 mutations are associated with an epileptic phenotype (see [724, 725]). In addition, epilepsy has also been reported in FAD mutations leading to duplications of APP [726]. Given these observations, it is not surprising that having the APPswe/PS1 $1 \mathrm{E} 9$ genotype significantly predicted the chance of developing epileptic seizures in our study. Yet, the observation that the occurrence of epileptic seizures in the APPswe/PS1 $\Delta \mathrm{E} 9$ mouse model can be triggered by exogenous factors, such as fluoxetine treatment, is of additional interest.

\subsection{A role for serotonin signaling in epilepsy?}

Certain factors may affect the seizure threshold. For example, a (family) history of seizures is a risk factor for antidepressant-induced seizures [727]. It can therefore be suggested that a decreased seizure threshold, e.g. due to genetic vulnerability, can be further diminished by antidepressants such as fluoxetine [728]. The current study supports this as the epilepticprone APPswe/PS1 $\triangle E 9$ mice had a much higher incidence of seizures after fluoxetine treatment compared to fluoxetine-treated WT mice. It has been suggested that only those with slow antidepressant metabolism are likely to develop seizures $[728,729]$. In this line, 
altered fluoxetine metabolism in APPswe/PS1 $\triangle \mathrm{E} 9$ could be considered as an underlying mechanism for their reduced seizure threshold. Although injections per se did not predict the onset of seizures, stress in general may have contributed to seizure development in these mice, particularly when combined with fluoxetine treatment. The observation that seizure behavior often initiated immediately prior to the fluoxetine injection, i.e. when the animal was fixated, supports this notion.

Markedly, antidepressants including SSRIs are at times prescribed to epilepsy patients for their anticonvulsant properties [730-732], particularly when the patient is suffering from comorbid depression [733]. Conversely, antidepressants can also lead to seizures [727, 734-736]. The relationship between antidepressants and seizure activity seems to be biphasic, where low levels of antidepressants are anticonvulsive and high levels are proconvulsive [727, 737]. Antiepileptic drugs show similar responses, as they are also able to induce seizures at high levels $[728,738]$. Paradoxically, fluoxetine is the SSRI with the lowest risk for seizure development, with a reported incidence rate of only $0.2 \%$ [727, 739].

It must be noted that fluoxetine is a highly promiscuous drug, not only binding the serotonin transporter (SERT), but various other receptors, including muscarinic, histaminic, dopaminergic receptors, and ion channels as well [740, 741]. As a matter of fact, fluoxetine has the highest affinity with SERT at low concentrations, while therapeutic concentrations are often 10-fold higher, i.e. within a range that preferentially targets the aforementioned receptors and ion channels $[740,742,743]$. Considering the observation that other druginduced seizures often arise from overdoses of antimuscarinic drugs or antihistamines [728, 741], one could speculate that the affinity of fluoxetine to bind to these receptors could underlie the observed induction of epileptic seizures in our mouse model.

Alternatively, several neurotransmitter systems, i.e. serotonin, noradrenalin, GABA and glutamate, have also been implicated in seizure susceptibility and are also compromised in the APPswe/PS1 $\triangle E 9$ mouse model. For example, serotonergic and/or noradrenergic deficits could contribute to seizure development $[744,745]$ and APPswe/PS1 $\triangle E 9$ mice show reduced monoamine levels, decreased $5 \mathrm{HT} 2 \mathrm{~A}$ receptor and SERT binding and global degeneration of monoaminergic afferents and neurons from 8 months of age [644, 746, 747]. Moreover, both AD patients and APPswe/PS1 1 E9 mice demonstrate that neurons in close proximity to plaques specifically lose their GABAergic perisomatic synapses [748], display hyperactive firing rates [749] and show calcium overload in their neurites, leading to neuritic degeneration [750]. As more plaques develop, more hyperactive neurons will form around these plaques [748], which could increase the chance to develop behavioral seizures. These neurotransmitter dysfunctions, specifically observed in APPswe/PS1 $\triangle E 9$ mice, could help explain their vulnerability to seizure development over WT mice.

\subsection{Considerations and recommendations}

To our knowledge this is the first report demonstrating epileptic seizures in a mouse model of $A D$ after chronic fluoxetine treatment. Others have performed 14 day fluoxetine treatment 
(intraperitoneally) of $\mathrm{Tg} 2576$ mice with $5 \mathrm{mg} / \mathrm{kg} / \mathrm{day}$, but reported no adverse events [78], possibly indicating that the PS1 mutation may indeed be a contributing factor in the current study. Alternatively, the difference in administered dose of fluoxetine may play a role as well in this respect. It should be noted that the dose of $15 \mathrm{mg} / \mathrm{kg}$ that we used, is well within the range that has been previously administered to mice, with chronic doses varying between 1 and $40 \mathrm{mg} / \mathrm{kg}$ [751-753]. However, fluoxetine has nonlinear pharmacokinetics and a long elimination half-life (1-4 days in humans, 13 hours in C57BL/6 mice) [754, 755], yet its active metabolite norfluoxetine has an ever greater elimination half-life and shows almost 2-fold higher concentrations at steady-state level than fluoxetine [754]. These pharmacokinetic characteristics may have resulted in sustainably high, and possibly accumulating, plasma (nor)fluoxetine levels in the APPswe/PS1 $\triangle \mathrm{E} 9$ mouse model particularly. Perhaps a lower dose of fluoxetine would have yielded more beneficial behavioral results, without inducing seizure activity. Furthermore, the high mortality rates significantly hindered assessing the behavioral effects of chronic fluoxetine treatment in APPswe/PS1 $\triangle E 9$ mice. These behavioral data should therefore be interpreted with care as they represent the survivors only and the low number of mice increased the variability in behavioral outcome.

Epidemiological data does underscore the beneficial effects of fluoxetine treatment for $A D$ patients. Depressed older $A D$ patients with a combined antidepressant and cholinergic inhibitor treatment had significantly less cognitive decline than patients treated with cholinergic inhibitors only [701]. Moreover, repeated use of antidepressants decreased the risk of developing $A D$ [700], and SSRI treatment improved memory and cognition in nondepressed mild cognitive impairment $(\mathrm{MCl})$ patients [756] and global cognitive function in $A D$ patients [702]. It was also shown that previous treatment with an SSRI reduced cortical binding of the Pittsburg compound, a PET radioligand selective for amyloid plaques, suggesting that serotonin signaling can regulate $A \beta$ production [703]. Indeed, in vivo studies verified that chronic treatment with the SSRI citalopram reduced $A \beta$ release into interstitial fluid in APPswe/PS1 $1 \mathrm{E} 9$ mice and decreased hippocampal plaque load [703]. These findings stress the importance of scrutinizing the mechanisms underlying fluoxetine's spcific ability to induce seizures.

Considering our current findings and the vast amount of literature on epileptic seizure susceptibility both in antidepressant medication as well as in AD patients, we believe one must be very careful when prescribing $A D$ patients with an antidepressant, particularly fluoxetine. $A D$ patients without clinical signs of depression are often treated with antidepressants, in order to manage behavioral and psychological symptoms of dementia (BPSD), such as agitation and aggression [757, 758]. Although many reports stress the benefits of antidepressant medication in $A D$ patients, and suggest these compounds are safe and effective options for the (complementary) treatment of $A D$ symptoms [701, 703, $705,759,760]$, we feel that these recommendations should be considered with care [760762]. In our view, it will be necessary to carefully monitor the plasma concentrations of fluoxetine in $A D$ patients, considering that antidepressant concentrations above therapeutical values have been associated with an increased risk for epilepsy [729], and AD 
patients have an increased susceptibility for epilepsy development [716-719]. Detailed evaluation of antidepressant metabolism may prevent unexpected occurrence of epileptic seizures in the AD population.

\subsection{Concluding remarks}

In conclusion, it can be said that fluoxetine treatment significantly increased the chance to develop seizures, specifically in APPswe/PS1 $\triangle \mathrm{E} 9$ mice. APPswe/PS1 $\Delta \mathrm{E} 9$ mice that have had seizures were also more likely to die prematurely. Importantly, epileptic seizure activity and high mortality rates have been observed previously for this mouse model, but never in conjunction with antidepressant treatment. Our observation that seizures can be induced by exogenous influences, such as drug treatment, is novel to the field. It would be interesting to investigate whether fluoxetine would also induce increased seizure activity in other $A D$ mouse models prone for seizure development, like the hAPPJ20 mouse [715]. Furthermore, clinical use of antidepressant medication in AD patients should be monitored with care, as these patients are at increased risk for developing epilepsy.

\section{Acknowledgements}

We thank Dr. Anja de Bruin (Solvay Pharmaceuticals, Weesp, the Netherlands) for kindly providing us with fluoxetine $\mathrm{HCl}^{-}$. 


\section{Chapter 9}

General Discussion 
Although genetic research over the past few decades has yielded great insights into the pathophysiology of Alzheimer's disease (AD), by now, it has become increasingly clear that genetic alterations alone cannot account for the vast majority of $A D$ cases. As such, it is likely that the environment plays a vital role in the onset and development of $A D$, either independently or synergistically with genetic factors. The aim of this thesis was to investigate the effects of stress exposure on the phenotypical expression of AD-related behavior and neuropathology, by using the APPswe/PS1 $1 \mathrm{E} 9$ mouse model of AD. Additionally, the ability of various pharmacological agents to alter disease progression was explored.

\section{Gene-environment interactions in $A D$ research}

Chapter 2 explored the concept of gene-environment interactions in helping to elucidate the etiopathogenesis of $A D$. It provided an extensive overview of several environmental exposures implicated in the onset or progression of $A D$, including chronic stress, environmental enrichment, exposure to metals and electromagnetic fields, traumatic brain injury, diet and nutrition. For every exposure, epidemiological data on the involvement of this factor in AD development was discussed (when available). However, as heterogeneity of the study population and interference from additional and uncontrollable environmental factors can confound the interpretation of epidemiological data, the effects of environmental factors in mouse models of $A D$ were also explored in depth. It was concluded that animal models, in particular mouse models, may be a useful tool to investigate the interactive as well as the independent effects of genes and the environment, attributable to the ability to strictly control environmental variables as well as the time- and region-specific expression of transgenes. Yet, the high level of variability in form, timing and onset of environmental exposure, as well as the age, sex and type of mice used, interfered with the ability to draw firm and general conclusions on the effects of the discussed environmental exposures. Although numerous challenges lie ahead in clarifying the precise role of gene-environment interactions in $A D$ onset, this concept could be considered a viable approach in identifying the exact biological underpinnings of this multifactorial disorder. Therefore, gene-environment interactions form the framework of the empirical research of this thesis.

\section{Part 1: Stress as a risk factor for $A D$}

In Part 1 of this thesis, the effects of chronic stress exposure on the onset and progression of AD-related cognitive decline and neuropathology were explored. As research had indicated that major depression (MD), a highly prevalent stress-related disorder, could be considered as a risk factor for $A D$ development, Chapter 3 reviewed the evidence for this disease interaction, the similarities between both illnesses in terms of neuromorphological alterations and the (interactive) role of the serotonergic system and hypothalamo-pituitaryadrenal (HPA) axis dysregulation in this respect. 


\section{Prenatal stress exposure induces sex-specific effects}

Although extensive research has been performed on the effects of chronic stress exposure, i.e. restraint, chronic isolation or chronic mild stress, during adolescence and adulthood, in various mouse models of $A D$, the long-lasting implications of gestational stress on $A D$ progression had not been explored. Perturbations during the prenatal period have shown to be critically involved in priming the offspring towards a psychopathological phenotype, including stress-related disorders like MD. As epigenetic regulation of gene expression (i.e. via modifications in DNA methylation and chromatin remodeling) strictly controls overall foetal development, and is also critically involved in mediating the effects of the developmental programming of adult health and disease, it could be speculated that perturbations during the prenatal period can have a long-lasting effect via epigenetic regulation on $A D$-related phenotypes later in life.

Chapters 4 and $\mathbf{5}$ explored these considerations, by mating C57BL/6 mothers with APPswe/PS1 $1 \mathrm{E} 9$ fathers and applying restraint stress to the pregnant dams during the first part of pregnancy. It was hypothesized that prenatal stress exposure would accelerate the onset of AD-related cognitive deficits and neuropathology in male and female APPswe/PS1 $1 \mathrm{E} 9$ offspring, possibly by altering global DNA methylation and hydroxymethylation patterns (Chapter 4). In addition, the role of brain-derived neurotrophic factor (BDNF) signaling was explored as altered expression of this neurotrophic factor is implicated both in MD and AD (Chapter 5). The data presented in these chapters indicated highly sex-specific effects of prenatal stress exposure. Male APPswe/PS1 $1 \mathrm{E} 9$ mice displayed more cognitive deficits after prenatal stress exposure and an altered HPA-axis response after an acute stressor, yet in terms of AD-related neuropathology or BDNF signaling, no effects of prenatal stress could be established. Alternatively, female APPswe/PS1 $1 \mathrm{E} 9$ mice showed relative protection from cognitive deficits after prenatal stress exposure, but also developed a more depressive-like phenotype. On the neurophysiological level, female APPswe/PS1 1 E9 mice exposed to prenatal stress had significant reductions in hippocampal plaque load compared to non-exposed females, which was rather unexpected, and might be linked to their improved cognitive performance. Moreover, prenatally stressed females displayed a decrease in mature BDNF (mBDNF) concentrations compared to control female mice. Low levels of mBDNF have been implicated in depressive-like behavior, a phenotype that is also displayed in the prenatallystressed females. Effects of prenatal stress on global DNA (hydroxy)methylation patterns could not established. On one hand, it could be hypothesized that alterations in epigenetic regulation may have a latency in their manifestation, as reported previously by Lahiri et al. [80], and will only become apparent at old age. Accordingly, epigenetic drift increases with aging [763], so perhaps read-out of our animals at a later age would have demonstrated prenatal stress-induced alterations in DNA (hydroxy)methylation patterns. Alternatively, it could be posed that the degree of (hydroxy)methylation has only changed at specific genes, while not affecting the global (hydroxy)methylation status in this mouse model. 


\section{Sex differences in APPswe/PS1 1 E9 mice}

Besides the sex-specific effects of prenatal stress exposure, the research described in Chapters 4 and 5 highlights prominent differences in the onset and progression of ADrelated phenotypes between males and females. Indeed, epidemiological studies imply that females may have a heightened risk towards $A D$ development $[5,43]$, although others state these results are confounded by the fact that women become older than men [764]. As age is the main risk factor for $A D$ development, women are statistically more inclined to develop this illness. Although further epidemiological research will be needed to clarify this issue, it remains an interesting observation that in many $A D$ mouse models, including APPswe/PS1 $1 \mathrm{E} 9$ mice, female mice display an earlier onset of cognitive deficits and neuropathological hallmarks $[289,579,580,765]$. The same findings were reported in Chapters 4 and 5 , as female APPswe/PS1 $1 \mathrm{E} 9$ mice had more pronounced cognitive deficits than male mice of the same age. Moreover, females displayed higher hippocampal plaque load and soluble $A \beta$ levels than males, yet displayed slightly lower levels of intraneuronal $A \beta$. This could indicate that female APPswe/PS1 $1 E 9$ mice generally produce more $A \beta$ and have higher $A \beta$ secretion into the extracellular space when compared to male mice. APPswe/PS1 $\triangle E 9$ females also showed significantly higher concentrations of hippocampal proBDNF than male mice, which in turn could increase activation of the pro-apoptotic p $75^{\text {NTR }}$. In addition, DNA methylation is differentially regulated in males and females, with female APPswe/PS1 $\triangle \mathrm{E} 9$ mice displaying lower DNA methyltransferase (DNMT) 3a levels in the hippocampal dentate gyrus (DG).

Sex hormones may play a crucial part in regulating several of these investigated variables. Indeed, estrogens may affect $A \beta$ production and clearance pathways [766] and play an important role in the regulation of BDNF concentrations [595], thereby possibly explaining the sex differences in $A \beta$ load and BDNF isoform expression. Additionally, regional expression of DNMT3a can be influenced by the absence or presence of specific sex hormones [767], yet may in turn also regulate expression of estrogen receptors [768].

\section{Part 2: Efficacy of drug treatment}

Part 2 of this thesis described the conducted research on the effects of pharmacological interventions in attenuating AD-related behavior and neuropathology, by using phosphodiesterase inhibitors (PDE-Is) and the antidepressant fluoxetine.

\section{Phosphodiesterase inhibitors as putative treatment for $A D$}

Acute and chronic treatment using PDE-Is can improve memory, as has been demonstrated previously in various species and across numerous cognitive tasks [657]. However, in those experiments the efficacy of PDE-ls was tested in models of natural forgetting or pharmacologically-induced memory impairments. As AD patients typically suffer from cognitive dysfunction, it was interesting to investigate the usefulness of these compounds in 
light of this disorder. To that extent, APPswe/PS1 $\triangle E 9$ mice received chronic PDE-I treatment, using the PDE2-I BAY60-7550 (Chapter 6) and the PDE4D-I GEBR-7b (Chapter 7). It can be concluded that both BAY60-7550 as well as GEBR-7b were effective in improving memory function in APPswe/PS1 $\triangle \mathrm{E} 9$ mice. Most importantly, the treatment was efficacious even after onset of neuropathology, making these compounds putative therapeutic options in clinical settings, as AD patients have normally accumulated extensive neuropathology before treatment is commenced. Moreover, the pharmacological profile of chronic PDE inhibition is limited to enhancing cognition, as no detrimental alterations were observed in weight, affective behavior or neuroendocrine regulation as a result of treatment.

Although others have pinpointed roles for elevations in CAMP responsive elementbinding (CREB) protein phosphorylation, increases in BDNF, reduction in $A \beta$ or altered tau phosphorylation as underlying mechanisms for the cognition-enhancing effects of PDE-ls $[66,67,632]$, we could not establish changes in these intra- and extracellular proteins after chronic BAY60-7550 or GEBR-7b treatment. It must be noted, however, that the expression of PDEs is highly region-specific within the brain [616], and even displays a high degree of compartmentalization within the cell [652]. Due to their isoform-specificity or cyclic nucleotide preference, different PDE-Is may exert divergent effects on cellular function and ultimately protein expression or behavior. In clinical settings, PDE-ls are mainly used for their vasodilatory function (e.g. the PDE5-I Viagra), and it could therefore be proposed that the cognition-enhancing effects of PDE-Is are purely a reflection of increased blood flow and nutrient transport. Attempts to elucidate this assumption by measuring cerebral blood flow and glucose utilization after rolipram (PDE4-I) and vardenafil (PDE5-I) treatment concluded that the improvements in memory function were independent from changes in cerebral perfusion [655]. More research will be needed to verify these findings for other PDE-Is as well, and to elucidate via which intracellular mechanisms chronic PDE2 and PDE4D inhibition can improve memory performance in APPswe/PS1 1 E9 mice.

\section{Use of fluoxetine in $A D$}

Antidepressant treatment is quite common in patients with $A D$, either to battle comorbid depression, or to manage the behavioral and psychological symptoms of dementia (BPSD) [758]. Indeed, approximately $30-40 \%$ of $A D$ patients will receive antidepressants during the course of their illness [769]. In Chapter 8, it was explored whether chronic treatment with the selective serotonin reuptake inhibitor (SSRI) fluoxetine could prevent memory decline -dependently or independently of changes in affective behavior- and whether it would reduce neuropathology in APPswe/PS1 $\triangle \mathrm{E} 9$ mice. Unfortunately, it was observed that almost half of all fluoxetine-treated APPswe/PS1 $\triangle \mathrm{E} 9$ mice developed behaviors reminiscent of epileptic seizures and treatment had to be ceased on ethical grounds. Moreover, within the same group, 7 out of 13 animals died. Although premature death has been reported previously for this mouse model [770], and spontaneous behavioral seizures can occur [712, 713], this is the first study to report induction of an epileptic phenotype in APPswe/PS1 $\triangle E 9$ 
mice in response to exogenous influences. Although fluoxetine is at times prescribed as an anticonvulsant [732], it can have pro-convulsive properties at high dosages [727]. Since AD patients are already more prone to develop epilepsy than the general population [716], these data suggest that caution should be used when prescribing fluoxetine to AD patients, by, for instance, strictly monitoring fluoxetine concentrations in blood plasma.

\section{Influence of chronic injections}

Part 1 of this thesis demonstrated that chronic stress exposure can alter AD-related behavior and neuropathology. In this respect, it must be noted that all mice in Chapter 6 received a daily injection, which has shown to be a stressful experience for rodents [771]. Moreover, recent rodent data from our group demonstrated that chronic injections can lead to marked reductions in hippocampal BDNF levels [667], and can even induce hippocampal cell death, as shown by others [772]. For this reason, it was questioned whether subtle differences in behavior or neuropathology due to BAY60-7550 treatment were obscured by effects of injection stress. To control for this possible confounder, it was decided to include a 'no injection' group in the pharmacological experiments described in Chapters 7 and 8, in order to assess whether receiving chronic injections would induce a more depressive-like phenotype or would prime the HPA axis towards an exaggerated response when faced with an acute stressor. Whereas Chapter 7 includes mice that received daily subcutaneous injections for 3 weeks, the mice described in chapter 8 were administered daily oral injections (through an oral gavage). To be able to assess the effects of chronic injections on affective behavior, the sucrose-intake test and the elevated zero maze, measuring depressive-like behavior and levels of anxiety, respectively, were performed shortly after the last injection. Interestingly, in both studies, receiving daily injections for 3 weeks had no significant effect on affective phenotype, cognitive performance or neuroendocrine regulation. Daily injections also did not alter hippocampal levels of $A \beta$, BDNF, BDNF receptors, $(p) C R E B,(p)$ tau or $(p) G S K 3 \beta$. We can therefore conclude that a 3-week injection paradigm in $\mathrm{C} 57 \mathrm{BL} / 6$ and $\mathrm{APPswe} / \mathrm{PS} 1 \Delta \mathrm{E} 9$ mice has no long-term consequences on behavior or intracellular processes in our chronic treatment studies.

\section{Synthesis and perspectives}

Part 1 of our research underscored the high degree of sex-specific variability in the APPswe/PS1 $1 \mathrm{E} 9$ mouse model of $A D$ in terms of cognitive decline and neuropathology. This sex-specific vulnerability could be explained either by an earlier age of onset or by a more progressive disease development. Based on the available literature, the first option seems most plausible [556], yet very few studies actually directly compare male and female APPswe/PS1 $1 \mathrm{E} 9$ mice in a longitudinal fashion, making it difficult to pinpoint the exact mechanistic difference. Further identification of underlying mechanisms for sex-specific differences in $A D$ manifestation is needed to ensure proper treatment of patient groups. 
It can be concluded that chronic (developmental) stress exposure can permanently impact on the onset and progression of AD-related behavior and pathology. Effects of prenatal stress exposure show a high degree of sexual differentiation, making it likely that similar patterns may be observed for other environmental exposures. Therefore, we recommend that the effects of variations in environmental exposure should be tested in both sexes before firm conclusions can be drawn and these findings are translated back to clinical situations.

For Part 2 of this thesis, it can be stated that certain pharmacological interventions may indeed postpone disease manifestation, as was inferred from Lahiri's two-hit latency model (see Chapter 1, page 18). By enhancing yet unidentified processes, chronic PDE inhibition may circumvent the detrimental properties of $A \beta$ species on memory, thereby ensuring a delay in the onset of symptoms. In other cases, such as fluoxetine treatment, the pharmacological intervention is not neuroprotective, but may in fact exacerbate expression of an otherwise dormant phenotype. PDE inhibition should be considered as a valid treatment strategy for $A D$, particularly when regarding that it has memory-enhancing efficacy even after onset of pathology, making the use of these drugs suitable for clinical application. Additionally, a recent genome-wide association study revealed that a SNP within the intron of PDE7A can affect the rate of cognitive decline [773], further substantiating exploration of PDE-ls as treatment for AD. However, more research must be performed before PDE-Is will become available as therapeutic options for $A D$. First of all, it must be determined which $\mathrm{PDE}(\mathrm{s})$ is (are) the best target to treat AD. Second, PDE-ls still need further screening for efficacy and possible side effects, also in females. Third, it will be important to elucidate the biological mechanisms underlying their therapeutic effects, not in the least to also gain more insight into the pathophysiology of dementia in general and $A D$ in particular. To our knowledge, three clinical phase II trials are currently ongoing to investigate the efficacy of PDE-ls in enhancing cognition in healthy controls as well as AD patients (clinicaltrials.gov; NCT01433666, NCT00362024 and NCT0930059), and their results are awaited with anticipation.

\section{Considerations}

Several considerations must be kept in mind. First of all, in Chapters 4 and 5 the effects of prenatal stress were not examined in wild-type (WT) animals, which could have aided in disentangling the synergistic participation of genes and environment in altering the behavioral phenotype. In these studies, however, we wanted to examine the additive effect of prenatal stress exposure on the progression of AD-related symptoms and neuropathology, by using a model in which alterations in amyloid processing or AD-related symptoms could be assessed. Addition of a WT group would not have provided us with additional information on these features. 
Second, although research on environmental exposures in mouse models of $A D$ can provide insights into factors advancing or delaying disease progression, the onset of ADrelated symptoms and pathology in these models is inevitably driven by the expression of transgenes. Therefore, to elucidate the role of environmental factors in initiating $A D$ manifestation, the generation of models of sporadic $A D$ is of vital importance, yet is complicated by the fact that no driving force behind sporadic AD has been identified to date, ending up in stalemate. Alternatives may include assessment of mammals naturally developing amyloidosis, like primates, dolphins or rabbits [774], or hibernating species temporarily forming tangle-like pathology, e.g. bears and hamsters [775]. Yet, assessment of these models may come with a new set of limitations, including availability of the model and use of validated cognitive tests [774].

Other considerations include the housing facilities of the animals. In all experiments, mice were housed individually, due to the aggressive behavior observed among male APPswe/PS1 $\triangle \mathrm{E} 9$ mice. Single housing is considered stressful to mice [771], as they are social animals, and this form of housing is also used as a chronic stress paradigm [78, 507]. Therefore, stress from solitary housing may have masked the effects of prenatal stress exposure or injection stress.

\section{Concluding remarks}

Overall, it can be concluded that alterations in the environment can impact upon AD-related cognitive decline and neuropathology. However, these effects have shown to be highly sexspecific, an observation that must be kept in mind when assessing environmental exposures in AD mouse models. In addition, it was shown that alterations in behavioral phenotype are not necessarily accompanied by changes in neuropathology, demonstrating the complexity between brain physiology and function in orchestrating behavioral outcome. Moreover, although more research will be needed to elucidate the underlying biochemical mechanisms, PDE-I treatment should be considered a suitable treatment option for AD. Conversely, the use of fluoxetine in $A D$ patients should, in the least, be carefully monitored.

More than 100 years after Alois Alzheimer's discovery, enormous insights into the pathophysiology of $A D$ have been gained. One can only wish it will not take another 100 years to answer the many unresolved questions, ultimately curing this enigmatic disease. 


\section{References}

1. Alzheimer, A., et al., An English translation of Alzheimer's 1907 paper, "Uber eine eigenartige Erkankung der Hirnrinde". Clin Anat, 1995. 8(6): p. 429-31.

2. Maurer, K., S. Volk, and H. Gerbaldo, Auguste $D$ and Alzheimer's disease. Lancet, 1997. 349(9064): p. 1546-9.

3. Dahm, R., Alzheimer: 100 years and beyond, ed. M. Jucker, et al. 2006: Springer Verlag.

4. Hippius, H. and G. Neundorfer, The discovery of Alzheimer's disease. Dialogues Clin Neurosci, 2003. 5(1): p. 101-8.

5. WHO, Dementia: a public health priority. 2012, World Health Organization.

6. Wimo, A., et al., The economic impact of dementia in Europe in 2008-cost estimates from the Eurocode project. Int J Geriatr Psychiatry, 2011. 26(8): p. 82532.

7. American Psychiatric Association. Task Force on DSM-IV. and American Psychiatric Association., Diagnostic and statistical manual of mental disorders : DSM-IV-TR. 4th ed. 2000, Washington, DC: American Psychiatric Association. xxxvii, $943 \mathrm{p}$.

8. Parnetti, L., et al., Pharmacological treatment of non-cognitive disturbances in dementia disorders. Mech Ageing Dev, 2001. 122(16): p. 2063-9.

9. Shinosaki, K., T. Nishikawa, and M. Takeda, Neurobiological basis of behavioral and psychological symptoms in dementia of the Alzheimer type. Psychiatry Clin Neurosci, 2000. 54(6): p. 611-20.

10. Fitzpatrick, A.L., et al., Survival following dementia onset: Alzheimer's disease and vascular dementia. J Neurol Sci, 2005. 229-230: p. 43-9.

11. Organization, W.H., The 10 leading causes of death by broad income group (2008). 2008.

12. Ganguli, M., et al., Alzheimer disease and mortality: a 15-year epidemiological study. Arch Neurol, 2005. 62(5): p. 779-84.

13. Kammoun, S., et al., Immediate causes of death of demented and non-demented elderly. Acta Neurologica Scandinavica, 2000. 102: p. 96-99.

14. Brunnstrom, H.R. and E.M. Englund, Cause of death in patients with dementia disorders. Eur J Neurol, 2009. 16(4): p. 488-92.

15. Ueki, A., et al., Factors associated with mortality in patients with early-onset Alzheimer's disease: a five-year longitudinal study. International Journal of Geriatric Psychiatry, 2001. 16(8): p. 810-815.

16. Forstl, H., et al., Brain atrophy in normal ageing and Alzheimer's disease. Volumetric discrimination and clinical correlations. Br J Psychiatry, 1995. 167(6): p. 739-46.

17. Tanabe, J.L., et al., Tissue segmentation of the brain in Alzheimer disease. AJNR Am J Neuroradiol, 1997. 18(1): p. 115-23.

18. Cullen, K.M., et al., Cell loss in the nucleus basalis is related to regional cortical atrophy in Alzheimer's disease. Neuroscience, 1997. 78(3): p. 641-52.

19. Regeur, L., et al., No global neocortical nerve cell loss in brains from patients with senile dementia of Alzheimer's type. Neurobiol Aging, 1994. 15(3): p. 347-52.

20. West, M.J., et al., Differences in the pattern of hippocampal neuronal loss in normal ageing and Alzheimer's disease. Lancet, 1994. 344(8925): p. 769-72. 
21. Simic, G., et al., Volume and number of neurons of the human hippocampal formation in normal aging and Alzheimer's disease. J Comp Neurol, 1997. 379(4): p. 482-94.

22. Morrison, J.H. and P.R. Hof, Life and death of neurons in the aging brain. Science, 1997. 278(5337): p. 412-9.

23. Lassmann, H., P. Fischer, and K. Jellinger, Synaptic pathology of Alzheimer's disease. Ann N Y Acad Sci, 1993. 695: p. 59-64.

24. Davies, C.A., et al., A quantitative morphometric analysis of the neuronal and synaptic content of the frontal and temporal cortex in patients with Alzheimer's disease. J Neurol Sci, 1987. 78(2): p. 151-64.

25. Terry, R.D., et al., Physical basis of cognitive alterations in Alzheimer's disease: synapse loss is the major correlate of cognitive impairment. Ann Neurol, 1991. 30(4): p. 572-80.

26. Spires-Jones, T.L., et al., Are tangles as toxic as they look? J Mol Neurosci, 2011. 45(3): p. 438-44.

27. Chasseigneaux, S. and B. Allinquant, Functions of Abeta, sAPPalpha and sAPPbeta : similarities and differences. J Neurochem, 2012. 120 Suppl 1: p. 99108.

28. Kandalepas, P.C. and R. Vassar, Identification and biology of beta-secretase. J Neurochem, 2012. 120 Suppl 1: p. 55-61.

29. De Strooper, B. and W. Annaert, Novel research horizons for presenilins and gamma-secretases in cell biology and disease. Annu Rev Cell Dev Biol, 2010. 26: p. 235-60.

30. Takami, M., et al., gamma-Secretase: successive tripeptide and tetrapeptide release from the transmembrane domain of beta-carboxyl terminal fragment. J Neurosci, 2009. 29(41): p. 13042-52.

31. Funamoto, S., et al., Truncated carboxyl-terminal fragments of beta-amyloid precursor protein are processed to amyloid beta-proteins 40 and 42 . Biochemistry, 2004. 43(42): p. 13532-40.

32. Hardy, J.A. and G.A. Higgins, Alzheimer's disease: the amyloid cascade hypothesis. Science, 1992. 256(5054): p. 184-5.

33. Dickson, D.W., The pathogenesis of senile plaques. J Neuropathol Exp Neurol, 1997. 56(4): p. 321-39.

34. Giannakopoulos, P., et al., Tangle and neuron numbers, but not amyloid load, predict cognitive status in Alzheimer's disease. Neurology, 2003. 60(9): p. 1495500 .

35. Maarouf, C.L., et al., Alzheimer's disease and non-demented high pathology control nonagenarians: comparing and contrasting the biochemistry of cognitively successful aging. PLoS One, 2011. 6(11): p. e27291.

36. Glabe, C.G., Structural classification of toxic amyloid oligomers. J Biol Chem, 2008. 283(44): p. 29639-43.

37. Wirths, O., G. Multhaup, and T.A. Bayer, A modified beta-amyloid hypothesis: intraneuronal accumulation of the beta-amyloid peptide--the first step of a fatal cascade. J Neurochem, 2004. 91(3): p. 513-20.

38. Winton, M.J., et al., Intraneuronal APP, not free Abeta peptides in 3xTg-AD mice: implications for tau versus Abeta-mediated Alzheimer neurodegeneration. J Neurosci, 2011. 31(21): p. 7691-9.

39. Benilova, I., E. Karran, and B. De Strooper, The toxic Abeta oligomer and Alzheimer's disease: an emperor in need of clothes. Nat Neurosci, 2012. 15(3): p. 349-57.

40. Ropper, A.H. and R.S. Williams, Relationship between plaques, tangles, and dementia in Down syndrome. Neurology, 1980. 30(6): p. 639-44. 
41. Selkoe, D.J., Alzheimer's disease: genes, proteins, and therapy. Physiol Rev, 2001. 81(2): p. 741-66.

42. Cruts, M. and C. Van Broeckhoven, Molecular genetics of Alzheimer's disease. Ann Med, 1998. 30(6): p. 560-5.

43. Gao, S., et al., The relationships between age, sex, and the incidence of dementia and Alzheimer disease: a meta-analysis. Arch Gen Psychiatry, 1998. 55(9): p. 80915.

44. Farrer, L.A., et al., Effects of age, sex, and ethnicity on the association between apolipoprotein E genotype and Alzheimer disease. A meta-analysis. APOE and Alzheimer Disease Meta Analysis Consortium. JAMA, 1997. 278(16): p. 1349-56.

45. Bertram, L., et al., Systematic meta-analyses of Alzheimer disease genetic association studies: the AlzGene database. Nat Genet, 2007. 39(1): p. 17-23.

46. Seshadri, S., et al., Genome-wide analysis of genetic loci associated with Alzheimer disease. JAMA, 2010. 303(18): p. 1832-40.

47. Jankowsky, J.L., et al., Co-expression of multiple transgenes in mouse CNS: a comparison of strategies. Biomol Eng, 2001. 17(6): p. 157-65.

48. Borchelt, D.R., et al., $A$ vector for expressing foreign genes in the brains and hearts of transgenic mice. Genet Anal, 1996. 13(6): p. 159-63.

49. Jankowsky, J.L., et al., Mutant presenilins specifically elevate the levels of the 42 residue beta-amyloid peptide in vivo: evidence for augmentation of a 42-specific gamma secretase. Hum Mol Genet, 2004. 13(2): p. 159-70.

50. Garcia-Alloza, M., et al., Characterization of amyloid deposition in the APPswe/PS1 $\triangle E 9$ mouse model of Alzheimer disease. Neurobiol Dis, 2006. 24(3): p. 516-24.

51. O'Leary, T.P. and R.E. Brown, Visuo-spatial learning and memory deficits on the Barnes maze in the 16-month-old APPswe/PS1 1 E9 mouse model of Alzheimer's disease. Behav Brain Res, 2009. 201(1): p. 120-7.

52. Reiserer, R.S., et al., Impaired spatial learning in the APPSwe + PSEN1DeltaE9 bigenic mouse model of Alzheimer's disease. Genes Brain Behav, 2007. 6(1): p. 54-65.

53. Scarpini, E., P. Scheltens, and H. Feldman, Treatment of Alzheimer's disease: current status and new perspectives. Lancet Neurol, 2003. 2(9): p. 539-47.

54. Cummings, J.L., Alzheimer's disease. N Engl J Med, 2004. 351(1): p. 56-67.

55. Reneerkens, O.A., et al., Selective phosphodiesterase inhibitors: a promising target for cognition enhancement. Psychopharmacology (Berl), 2009. 202(1-3): p. 419-43.

56. Bender, A.T. and J.A. Beavo, Cyclic nucleotide phosphodiesterases: molecular regulation to clinical use. Pharmacol Rev, 2006. 58(3): p. 488-520.

57. Scott Bitner, R., Cyclic AMP response element-binding protein (CREB) phosphorylation: A mechanistic marker in the development of memory enhancing Alzheimer's disease therapeutics. Biochem Pharmacol, 2011. 83(6): p. 705-14.

58. Lu, Y.F., E.R. Kandel, and R.D. Hawkins, Nitric oxide signaling contributes to latephase LTP and CREB phosphorylation in the hippocampus. J Neurosci, 1999. 19(23): p. 10250-61.

59. Bliss, T.V. and G.L. Collingridge, A synaptic model of memory: long-term potentiation in the hippocampus. Nature, 1993. 361(6407): p. 31-9.

60. Frey, U., Y.Y. Huang, and E.R. Kandel, Effects of cAMP simulate a late stage of LTP in hippocampal CA1 neurons. Science, 1993. 260(5114): p. 1661-4.

61. Vitolo, O.V., et al., Amyloid beta -peptide inhibition of the PKA/CREB pathway and long-term potentiation: reversibility by drugs that enhance cAMP signaling. Proc Natl Acad Sci U S A, 2002. 99(20): p. 13217-21.

62. Venturini, G., et al., Beta-amyloid inhibits NOS activity by subtracting NADPH availability. FASEB J, 2002. 16(14): p. 1970-2. 
63. Miller, T.W., et al., Amyloid-beta inhibits No-cGMP signaling in a CD36- and CD47dependent manner. PLoS One, 2011. 5(12): p. e15686.

64. Monfort, P. and V. Felipo, Amyloid-beta impairs, and ibuprofen restores, the cGMP pathway, synaptic expression of AMPA receptors and long-term potentiation in the hippocampus. J Alzheimers Dis, 2010. 22(3): p. 795-809.

65. Comery, T.A., et al., Acute gamma-secretase inhibition improves contextual fear conditioning in the Tg2576 mouse model of Alzheimer's disease. J Neurosci, 2005. 25(39): p. 8898-902.

66. Gong, B., et al., Persistent improvement in synaptic and cognitive functions in an Alzheimer mouse model after rolipram treatment. J Clin Invest, 2004. 114(11): p. 1624-34.

67. Puzzo, D., et al., Phosphodiesterase 5 inhibition improves synaptic function, memory, and amyloid-beta load in an Alzheimer's disease mouse model. J Neurosci, 2009. 29(25): p. 8075-86.

68. Robichaud, A., et al., Assessing the emetic potential of PDE4 inhibitors in rats. $\mathrm{Br} \mathrm{J}$ Pharmacol, 2002. 135(1): p. 113-8.

69. Reyes-Irisarri, E., et al., Expression of the cGMP-specific phosphodiesterases 2 and 9 in normal and Alzheimer's disease human brains. Eur J Neurosci, 2007. 25(11): p. 3332-8.

70. Puzzo, D., et al., Amyloid-beta peptide inhibits activation of the nitric oxide/CGMP/CAMP-responsive element-binding protein pathway during hippocampal synaptic plasticity. J Neurosci, 2005. 25(29): p. 6887-97.

71. Grunblatt, E., et al., Genetic risk factors and markers for Alzheimer's disease and/or depression in the VITA study. J Psychiatr Res, 2009. 43(3): p. 298-308.

72. Wilson, R.S., et al., Chronic psychological distress and risk of Alzheimer's disease in old age. Neuroepidemiology, 2006. 27(3): p. 143-53.

73. Wilson, R.S., et al., Proneness to psychological distress is associated with risk of Alzheimer's disease. Neurology, 2003. 61(11): p. 1479-85.

74. Geerlings, M.I., et al., History of depression, depressive symptoms, and medial temporal lobe atrophy and the risk of Alzheimer disease. Neurology, 2008. 70(15): p. 1258-64.

75. Mejia, S., et al., Nongenetic factors as modifiers of the age of onset of familial Alzheimer's disease. Int Psychogeriatr, 2003. 15(4): p. 337-49.

76. Carroll, J.C., et al., Chronic stress exacerbates tau pathology, neurodegeneration, and cognitive performance through a corticotropin-releasing factor receptordependent mechanism in a transgenic mouse model of tauopathy. J Neurosci, 2011. 31(40): p. 14436-49.

77. Rothman, S.M., et al., 3xTgAD mice exhibit altered behavior and elevated Abeta after chronic mild social stress. Neurobiol Aging, 2011.

78. Dong, $\mathrm{H}$., et al., Modulation of hippocampal cell proliferation, memory, and amyloid plaque deposition in APPsw (Tg2576) mutant mice by isolation stress. Neuroscience, 2004. 127(3): p. 601-9.

79. van Os, J., B.P. Rutten, and R. Poulton, Gene-environment interactions in schizophrenia: review of epidemiological findings and future directions. Schizophr Bull, 2008. 34(6): p. 1066-82.

80. Lahiri, D.K., B. Maloney, and N.H. Zawia, The LEARn model: an epigenetic explanation for idiopathic neurobiological diseases. Mol Psychiatry, 2009. 14(11): p. 992-1003.

81. Wu, J., et al., Alzheimer's disease (AD)-like pathology in aged monkeys after infantile exposure to environmental metal lead $(P b)$ : evidence for a developmental origin and environmental link for $A D$. J Neurosci, 2008. 28(1): p. 3-9. 
82. Basha, M.R., et al., The fetal basis of amyloidogenesis: exposure to lead and latent overexpression of amyloid precursor protein and beta-amyloid in the aging brain. $\mathrm{J}$ Neurosci, 2005. 25(4): p. 823-9.

83. Seshadri, S. and P.A. Wolf, Lifetime risk of stroke and dementia: current concepts, and estimates from the Framingham Study. Lancet Neurol, 2007. 6(12): p. 1106-14.

84. Querfurth, H.W. and F.M. LaFerla, Alzheimer's disease. N Engl J Med, 2010. 362(4): p. 329-44.

85. Pedersen, N.L., Reaching the limits of genome-wide significance in Alzheimer disease: back to the environment. J Am Med Assoc, 2010. 303(18): p. 1864-5.

86. EU-GEI, Schizophrenia aetiology: do gene-environment interactions hold the key? Schizophr Res, 2008. 102(1-3): p. 21-6.

87. van Os, J. and B.P. Rutten, Gene-environment-wide interaction studies in psychiatry. Am J Psychiatry, 2009. 166(9): p. 964-6.

88. Gatz, M., et al., Role of genes and environments for explaining Alzheimer disease. Arch Gen Psychiatry, 2006. 63(2): p. 168-74.

89. Raiha, I., et al., Alzheimer's disease in Finnish twins. Lancet, 1996. 347(9001): p. 573-8.

90. Raiha, I., et al., Environmental differences in twin pairs discordant for Alzheimer's disease. J Neurol Neurosurg Psychiatry, 1998. 65(5): p. 785-7.

91. Pedersen, N.L., et al., How heritable is Alzheimer's disease late in life? Findings from Swedish twins. Ann Neurol, 2004. 55(2): p. 180-5.

92. Harold, D., et al., Genome-wide association study identifies variants at CLU and PICALM associated with Alzheimer's disease. Nat Genet, 2009. 41(10): p. 1088-93.

93. Lambert, J.C., et al., Genome-wide association study identifies variants at CLU and CR1 associated with Alzheimer's disease. Nat Genet, 2009. 41(10): p. 1094-9.

94. Laumet, G., et al., Systematic analysis of candidate genes for Alzheimer's disease in a French, genome-wide association study. J Alzheimers Dis, 2010. 20(4): p. 1181-8.

95. Ganguli, M. and W.A. Kukull, Lost in translation: epidemiology, risk, and Alzheimer disease. Arch Neurol, 2010. 67(1): p. 107-11.

96. Migliore, L. and F. Coppede, Genetics, environmental factors and the emerging role of epigenetics in neurodegenerative diseases. Mutat Res, 2009. 667(1-2): p. 82-97.

97. Qu, C., D. De Ronchi, and L. Fratiglioni, The epidemiology of the dementias: an update. Curr Opin Psychiatry, 2007. 20(4): p. 380-5.

98. Chouliaras, L., et al., Epigenetic regulation in the pathophysiology of Alzheimer's disease. Prog Neurobiol, 2010. 90(4): p. 498-510.

99. Chandra, V. and R. Pandav, Gene-environment interaction in Alzheimer's disease: a potential role for cholesterol. Neuroepidemiology, 1998. 17(5): p. 225-32.

100. Jarvik, G.P., et al., Interactions of apolipoprotein E genotype, total cholesterol level, age, and sex in prediction of Alzheimer's disease: a case-control study. Neurology, 1995. 45(6): p. 1092-6.

101. Elbaz, A., C. Dufouil, and A. Alperovitch, Interaction between genes and environment in neurodegenerative diseases. C R Biol, 2007. 330(4): p. 318-28.

102. Dufouil, C., et al., Influence of apolipoprotein $E$ genotype on the risk of cognitive deterioration in moderate drinkers and smokers. Epidemiology, 2000. 11(3): p. 2804.

103. Hakansson, K., et al., Association between mid-life marital status and cognitive function in later life: population based cohort study. Br Med J, 2009. 339: p. b2462.

104. Basha, M.R., et al., Lead $(P b)$ exposure and its effect on APP proteolysis and Abeta aggregation, in FASEB J. 2005. p. 2083-4.

105. Carroll, B.J. and G.C. Curtis, Neuroendocrine identification of depressed patients. Aust N Z J Psychiatry, 1976. 10(1): p. 13-20. 
106. Csernansky, J.G., et al., Plasma cortisol and progression of dementia in subjects with Alzheimer-type dementia. Am J Psychiatry, 2006. 163(12): p. 2164-9.

107. Weiner, M.F., et al., Cortisol secretion and Alzheimer's disease progression. Biol Psychiatry, 1997. 42(11): p. 1030-8.

108. Joels, M., et al., Effects of chronic stress on structure and cell function in rat hippocampus and hypothalamus. Stress, 2004. 7(4): p. 221-31.

109. Magarinos, A.M., et al., Chronic psychosocial stress causes apical dendritic atrophy of hippocampal CA3 pyramidal neurons in subordinate tree shrews. J Neurosci, 1996. 16(10): p. 3534-40.

110. Sapolsky, R.M., A mechanism for glucocorticoid toxicity in the hippocampus: increased neuronal vulnerability to metabolic insults. J Neurosci, 1985. 5(5): p. 1228-32.

111. Sapolsky, R.M., L.C. Krey, and B.S. McEwen, Prolonged glucocorticoid exposure reduces hippocampal neuron number: implications for aging. J Neurosci, 1985. 5(5): p. 1222-7.

112. Alfarez, D.N., M. Joels, and H.J. Krugers, Chronic unpredictable stress impairs long-term potentiation in rat hippocampal CA1 area and dentate gyrus in vitro. Eur J Neurosci, 2003. 17(9): p. 1928-34.

113. Gould, E., et al., Neurogenesis in the dentate gyrus of the adult tree shrew is regulated by psychosocial stress and NMDA receptor activation. J Neurosci, 1997. 17(7): p. 2492-8.

114. Sierksma, A.S., et al., Major depression, cognitive dysfunction and Alzheimer's disease: is there a link? Eur J Pharmacol, 2010. 626(1): p. 72-82.

115. Caraci, F., et al., Depression and Alzheimer's disease: neurobiological links and common pharmacological targets. Eur J Pharmacol, 2010. 626(1): p. 64-71.

116. Rothman, S.M. and M.P. Mattson, Adverse stress, hippocampal networks, and Alzheimer's disease. Neuromolecular Med, 2010. 12(1): p. 56-70.

117. Geerlings, M.I., et al., Depression and risk of cognitive decline and Alzheimer's disease. Results of two prospective community-based studies in The Netherlands. Br J Psychiatry, 2000. 176: p. 568-75.

118. Green, R.C., et al., Depression as a risk factor for Alzheimer disease: the MIRAGE Study. Arch Neurol, 2003. 60(5): p. 753-9.

119. Grunblatt, E., et al., Tryptophan is a marker of human postmortem brain tissue quality. J Neurochem, 2009. 110(5): p. 1400-8.

120. Devanand, D.P., et al., Depressed mood and the incidence of Alzheimer's disease in the elderly living in the community. Arch Gen Psychiatry, 1996. 53(2): p. 175-82.

121. Dotson, V.M., M.A. Beydoun, and A.B. Zonderman, Recurrent depressive symptoms and the incidence of dementia and mild cognitive impairment. Neurology, 2010. 75(1): p. 27-34.

122. Saczynski, J.S., et al., Depressive symptoms and risk of dementia: the Framingham Heart Study. Neurology, 2010. 75(1): p. 35-41.

123. Wilson, R.S., et al., Temporal course of depressive symptoms during the development of Alzheimer disease. Neurology, 2010. 75(1): p. 21-6.

124. Ownby, D.R., C.C. Johnson, and E.L. Peterson, Incidence and prevalence of physician-diagnosed asthma in a suburban population of young adults. Ann Allergy Asthma Immunol, 1996. 77(4): p. 304-8.

125. Sun, X., et al., Depression and plasma amyloid beta peptides in the elderly with and without the apolipoprotein E4 allele. Alzheimer Dis Assoc Disord, 2009. 23(3): p. 238-44.

126. van Oijen, M., et al., Plasma Abeta(1-40) and Abeta(1-42) and the risk of dementia: a prospective case-cohort study. Lancet Neurol, 2006. 5(8): p. 655-60. 
127. Lavretsky, H., et al., Depression and anxiety symptoms are associated with cerebral FDDNP-PET binding in middle-aged and older nondemented adults. Am J Geriatr Psychiatry, 2009. 17(6): p. 493-502.

128. Rapp, M.A., et al., Increased hippocampal plaques and tangles in patients with Alzheimer disease with a lifetime history of major depression. Arch Gen Psychiatry, 2006. 63(2): p. 161-7.

129. Geerlings, M.I., et al., Depressive symptoms and risk of Alzheimer's disease in more highly educated older people. J Am Geriatr Soc, 2000. 48(9): p. 1092-7.

130. Swaab, D.F., A.M. Bao, and P.J. Lucassen, The stress system in the human brain in depression and neurodegeneration. Ageing Res Rev, 2005. 4(2): p. 141-94.

131. Dong, $\mathrm{H}$., et al., Corticosterone and related receptor expression are associated with increased beta-amyloid plaques in isolated Tg2576 mice. Neuroscience, 2008. 155(1): p. 154-63.

132. Kang, J.E., et al., Acute stress increases interstitial fluid amyloid-beta via corticotropin-releasing factor and neuronal activity. Proc Natl Acad Sci U S A, 2007. 104(25): p. 10673-8.

133. Pedersen, W.A., et al., Aberrant stress response associated with severe hypoglycemia in a transgenic mouse model of Alzheimer's disease. J Mol Neurosci, 1999. 13(1-2): p. 159-65.

134. Jeong, Y.H., et al., Chronic stress accelerates learning and memory impairments and increases amyloid deposition in APPV717I-CT100 transgenic mice, an Alzheimer's disease model. FASEB J, 2006. 20(6): p. 729-31.

135. Kunimoto, S., et al., Chronic stress-mutated presenilin 1 gene interaction perturbs neurogenesis and accelerates neurodegeneration. Exp Neurol, 2010. 221(1): p. 175-85.

136. Lee, K.W., et al., Behavioral stress accelerates plaque pathogenesis in the brain of Tg2576 mice via generation of metabolic oxidative stress. J Neurochem, 2009. 108(1): p. 165-75.

137. Green, K.N., et al., Glucocorticoids increase amyloid-beta and tau pathology in a mouse model of Alzheimer's disease. J Neurosci, 2006. 26(35): p. 9047-56.

138. Boyle, P.A., et al., Effect of a purpose in life on risk of incident Alzheimer disease and mild cognitive impairment in community-dwelling older persons. Arch Gen Psychiatry, 2010. 67(3): p. 304-10.

139. Laurin, D., et al., Physical activity and risk of cognitive impairment and dementia in elderly persons. Arch Neurol, 2001. 58(3): p. 498-504.

140. Krech, D., M.R. Rosenzweig, and E.L. Bennett, Effects of environmental complexity and training on brain chemistry. J Comp Physiol Psychol, 1960. 53: p. 509-19.

141. Nithianantharajah, J. and A.J. Hannan, The neurobiology of brain and cognitive reserve: mental and physical activity as modulators of brain disorders. Prog Neurobiol, 2009. 89(4): p. 369-82.

142. Adlard, P.A., et al., Voluntary exercise decreases amyloid load in a transgenic model of Alzheimer's disease. J Neurosci, 2005. 25(17): p. 4217-21.

143. Arendash, G.W., et al., Environmental enrichment improves cognition in aged Alzheimer's transgenic mice despite stable beta-amyloid deposition. Neuroreport, 2004. 15(11): p. 1751-4.

144. Herring, A., et al., Environmental enrichment counteracts Alzheimer's neurovascular dysfunction in TgCRND8 mice. Brain Pathol, 2008. 18(1): p. 32-9.

145. Herring, A., et al., Environmental enrichment enhances cellular plasticity in transgenic mice with Alzheimer-like pathology. Exp Neurol, 2009. 216(1): p. 18492.

146. Lewejohann, L., et al., Transgenic Alzheimer mice in a semi-naturalistic environment: more plaques, yet not compromised in daily life. Behav Brain Res, 2009. 201(1): p. 99-102. 
147. Gortz, N., et al., Effects of environmental enrichment on exploration, anxiety, and memory in female TgCRND8 Alzheimer mice. Behav Brain Res, 2008. 191(1): p. 43-8.

148. Wolf, S.A., et al., Cognitive and physical activity differently modulate disease progression in the amyloid precursor protein (APP)-23 model of Alzheimer's disease. Biol Psychiatry, 2006. 60(12): p. 1314-23.

149. Mirochnic, S., et al., Age effects on the regulation of adult hippocampal neurogenesis by physical activity and environmental enrichment in the APP23 mouse model of Alzheimer disease. Hippocampus, 2009. 19(10): p. 1008-18.

150. Cracchiolo, J.R., et al., Enhanced cognitive activity--over and above social or physical activity--is required to protect Alzheimer's mice against cognitive impairment, reduce Abeta deposition, and increase synaptic immunoreactivity. Neurobiol Learn Mem, 2007. 88(3): p. 277-94.

151. Lazarov, O., et al., Environmental enrichment reduces Abeta levels and amyloid deposition in transgenic mice. Cell, 2005. 120(5): p. 701-13.

152. Costa, D.A., et al., Enrichment improves cognition in $A D$ mice by amyloid-related and unrelated mechanisms. Neurobiol Aging, 2007. 28(6): p. 831-44.

153. Jankowsky, J.L., et al., Environmental enrichment exacerbates amyloid plaque formation in a transgenic mouse model of Alzheimer disease. J Neuropathol Exp Neurol, 2003. 62(12): p. 1220-7.

154. Jankowsky, J.L., et al., Environmental enrichment mitigates cognitive deficits in a mouse model of Alzheimer's disease. J Neurosci, 2005. 25(21): p. 5217-24.

155. Levi, O., et al., ApoE4 impairs hippocampal plasticity isoform-specifically and blocks the environmental stimulation of synaptogenesis and memory. Neurobiol Dis, 2003. 13(3): p. 273-82.

156. French, L.R., A.N. Williams, and R.G. Campbell, Lead toxicity. Minn Med, 1985. 68(12): p. 899-901.

157. Gun, R.T., et al., Occupational risk factors for Alzheimer disease: a case-control study. Alzheimer Dis Assoc Disord, 1997. 11(1): p. 21-7.

158. Santibanez, M., F. Bolumar, and A.M. Garcia, Occupational risk factors in Alzheimer's disease: a review assessing the quality of published epidemiological studies. Occup Environ Med, 2007. 64(11): p. 723-32.

159. Flaten, T.P., Aluminium as a risk factor in Alzheimer's disease, with emphasis on drinking water. Brain Res Bull, 2001. 55(2): p. 187-96.

160. Rogers, M.A. and D.G. Simon, A preliminary study of dietary aluminium intake and risk of Alzheimer's disease. Age Ageing, 1999. 28(2): p. 205-9.

161. Moore, P.B., et al., Absorption of aluminium-26 in Alzheimer's disease, measured using accelerator mass spectrometry. Dement Geriatr Cogn Disord, 2000. 11(2): $p$. 66-9.

162. Taylor, G.A., et al., Gastrointestinal absorption of aluminium in Alzheimer's disease: response to aluminium citrate. Age Ageing, 1992. 21(2): p. 81-90.

163. Pratico, D., et al., Aluminum modulates brain amyloidosis through oxidative stress in APP transgenic mice. FASEB J, 2002. 16(9): p. 1138-40.

164. Garcia, T., et al., Oxidative stress status and RNA expression in hippocampus of an animal model of Alzheimer's disease after chronic exposure to aluminum. Hippocampus, 2010. 20(1): p. 218-25.

165. Ribes, D., et al., Effects of oral aluminum exposure on behavior and neurogenesis in a transgenic mouse model of Alzheimer's disease. Exp Neurol, 2008. 214(2): p. 293-300.

166. Gomez, M., et al., Aluminum exposure through the diet: metal levels in AbetaPP transgenic mice, a model for Alzheimer's disease. Toxicology, 2008. 249(2-3): p. 214-9. 
167. Bush, A.I., et al., Modulation of $A$ beta adhesiveness and secretase site cleavage by zinc. J Biol Chem, 1994. 269(16): p. 12152-8.

168. Miura, T., et al., Metal binding modes of Alzheimer's amyloid beta-peptide in insoluble aggregates and soluble complexes. Biochemistry, 2000. 39(23): p. 702431.

169. Ricchelli, F., et al., Aluminum-triggered structural modifications and aggregation of beta-amyloids. Cell Mol Life Sci, 2005. 62(15): p. 1724-33.

170. Dwyer, B.E., et al., Getting the iron out: phlebotomy for Alzheimer's disease? Med Hypotheses, 2009. 72(5): p. 504-9.

171. Bartzokis, G., et al., In vivo evaluation of brain iron in Alzheimer disease using magnetic resonance imaging. Arch Gen Psychiatry, 2000. 57(1): p. 47-53.

172. Bartzokis, G., et al., Brain ferritin iron as a risk factor for age at onset in neurodegenerative diseases. Ann N Y Acad Sci, 2004. 1012: p. 224-36.

173. Deibel, M.A., W.D. Ehmann, and W.R. Markesbery, Copper, iron, and zinc imbalances in severely degenerated brain regions in Alzheimer's disease: possible relation to oxidative stress. J Neurol Sci, 1996. 143(1-2): p. 137-42.

174. Lovell, M.A., et al., Copper, iron and zinc in Alzheimer's disease senile plaques. J Neurol Sci, 1998. 158(1): p. 47-52.

175. Carlson, E.S., et al., Iron deficiency alters expression of genes implicated in Alzheimer disease pathogenesis. Brain Res, 2008. 1237: p. 75-83.

176. Jorgenson, L.A., et al., Fetal iron deficiency disrupts the maturation of synaptic function and efficacy in area CA1 of the developing rat hippocampus. Hippocampus, 2005. 15(8): p. 1094-102.

177. Jorgenson, L.A., J.D. Wobken, and M.K. Georgieff, Perinatal iron deficiency alters apical dendritic growth in hippocampal CA1 pyramidal neurons. Dev Neurosci, 2003. 25(6): p. 412-20.

178. Fernandez, L.L., et al., Effects of increased iron intake during the neonatal period on the brain of adult AbetaPP/PS1 transgenic mice. J Alzheimers Dis, 2010. 19(3): p. 1069-80.

179. Moreira, P., et al., Effect of zinc ions on the cytotoxicity induced by the amyloid beta-peptide. Antioxid Redox Signal, 2000. 2(2): p. 317-25.

180. Carson, J.A. and A.J. Turner, Beta-amyloid catabolism: roles for neprilysin (NEP) and other metallopeptidases? J Neurochem, 2002. 81(1): p. 1-8.

181. Lovell, M.A., C. Xie, and W.R. Markesbery, Protection against amyloid beta peptide toxicity by zinc. Brain Res, 1999. 823(1-2): p. 88-95.

182. Molina, J.A., et al., Cerebrospinal fluid levels of transition metals in patients with Alzheimer's disease. J Neural Transm, 1998. 105(4-5): p. 479-88.

183. Yang, D.S., et al., Examining the zinc binding site of the amyloid-beta peptide. Eur J Biochem, 2000. 267(22): p. 6692-8.

184. Stoltenberg, M., et al., Amyloid plaques arise from zinc-enriched cortical layers in APP/PS1 transgenic mice and are paradoxically enlarged with dietary zinc deficiency. Neuroscience, 2007. 150(2): p. 357-69.

185. Linkous, D.H., et al., The effects of enhanced zinc on spatial memory and plaque formation in transgenic mice. J Alzheimers Dis, 2009. 18(3): p. 565-79.

186. Maynard, C.J., et al., Chronic exposure to high levels of zinc or copper has little effect on brain metal homeostasis or Abeta accumulation in transgenic APP-C100 mice. Cell Mol Neurobiol, 2009. 29(5): p. 757-67.

187. Lee, J.Y., et al., Contribution by synaptic zinc to the gender-disparate plaque formation in human Swedish mutant APP transgenic mice. Proc Natl Acad Sci U S A, 2002. 99(11): p. 7705-10.

188. Kessler, H., et al., Effect of copper intake on CSF parameters in patients with mild Alzheimer's disease: a pilot phase 2 clinical trial. J Neural Transm, 2008. 115(12): p. 1651-9. 
189. Sparks, D.L. and B.G. Schreurs, Trace amounts of copper in water induce betaamyloid plaques and learning deficits in a rabbit model of Alzheimer's disease. Proc Natl Acad Sci U S A, 2003. 100(19): p. 11065-9.

190. Schafer, S., et al., Copper and clioquinol treatment in young APP transgenic and wild-type mice: effects on life expectancy, body weight, and metal-ion levels. J Mol Med, 2007. 85(4): p. 405-13.

191. Kitazawa, M., D. Cheng, and F.M. Laferla, Chronic copper exposure exacerbates both amyloid and tau pathology and selectively dysregulates cdk5 in a mouse model of AD. J Neurochem, 2009. 108(6): p. 1550-60.

192. Maynard, C.J., et al., Overexpression of Alzheimer's disease amyloid-beta opposes the age-dependent elevations of brain copper and iron. J Biol Chem, 2002. 277(47): p. 44670-6.

193. White, A.R., et al., Copper levels are increased in the cerebral cortex and liver of APP and APLP2 knockout mice. Brain Res, 1999. 842(2): p. 439-44.

194. Johnson, V.E., W. Stewart, and D.H. Smith, Traumatic brain injury and amyloid[beta] pathology: a link to Alzheimer's disease? Nat Rev Neurosci, 2010. 11(5): p. 361-370.

195. Johnson, V.E., et al., A neprilysin polymorphism and amyloid-beta plaques after traumatic brain injury. J Neurotrauma, 2009. 26(8): p. 1197-202.

196. Murai, H., et al., Twofold overexpression of human beta-amyloid precursor proteins in transgenic mice does not affect the neuromotor, cognitive, or neurodegenerative sequelae following experimental brain injury. J Comp Neurol, 1998. 392(4): p. 42838.

197. Smith, D.H., et al., Brain trauma induces massive hippocampal neuron death linked to a surge in beta-amyloid levels in mice overexpressing mutant amyloid precursor protein. Am J Pathol, 1998. 153(3): p. 1005-10.

198. Abrahamson, E.E., et al., Caspase inhibition therapy abolishes brain traumainduced increases in Abeta peptide: implications for clinical outcome. Exp Neurol, 2006. 197(2): p. 437-50.

199. Abrahamson, E.E., et al., Simvastatin therapy prevents brain trauma-induced increases in beta-amyloid peptide levels. Ann Neurol, 2009. 66(3): p. 407-14.

200. Crawford, F.C., et al., Genomic analysis of response to traumatic brain injury in a mouse model of Alzheimer's disease (APPsw). Brain Res, 2007. 1185: p. 45-58.

201. Uryu, K., et al., Repetitive mild brain trauma accelerates Abeta deposition, lipid peroxidation, and cognitive impairment in a transgenic mouse model of Alzheimer amyloidosis. J Neurosci, 2002. 22(2): p. 446-54.

202. Ferguson, S., et al., Apolipoprotein E genotype and oxidative stress response to traumatic brain injury. Neuroscience, 2010. 168(3): p. 811-9.

203. Arendash, G.W., et al., Electromagnetic field treatment protects against and reverses cognitive impairment in Alzheimer's disease mice. J Alzheimers Dis, 2010. 19(1): p. 191-210.

204. Soderqvist, F., et al., Radiofrequency fields, transthyretin, and Alzheimer's disease. J Alzheimers Dis, 2010. 20(2): p. 599-606.

205. Serot, J.M., et al., Cerebrospinal fluid transthyretin: aging and late onset Alzheimer's disease. J Neurol Neurosurg Psychiatry, 1997. 63(4): p. 506-8.

206. Galvin, J.E., Pass the grain; spare the brain. Neurology, 2007. 69(11): p. 1072-3.

207. Scarmeas, N., et al., Mediterranean diet, Alzheimer disease, and vascular mediation. Arch Neurol, 2006. 63(12): p. 1709-17.

208. Scarmeas, N., et al., Mediterranean diet and mild cognitive impairment. Arch Neurol, 2009. 66(2): p. 216-25.

209. Kivipelto, M., et al., Obesity and vascular risk factors at midlife and the risk of dementia and Alzheimer disease. Arch Neurol, 2005. 62(10): p. 1556-60. 
210. Luchsinger, J.A., et al., Caloric intake and the risk of Alzheimer disease. Arch Neurol, 2002. 59(8): p. 1258-63.

211. Pedrini, S., et al., Dietary composition modulates brain mass and solubilizable Abeta levels in a mouse model of aggressive Alzheimer's amyloid pathology. Mol Neurodegener, 2009. 4: p. 40.

212. Ho, L., et al., Diet-induced insulin resistance promotes amyloidosis in a transgenic mouse model of Alzheimer's disease. FASEB J, 2004. 18(7): p. 902-4.

213. Studzinski, C.M., et al., Effects of short-term Western diet on cerebral oxidative stress and diabetes related factors in APP x PS1 knock-in mice. J Neurochem, 2009. 108(4): p. 860-6.

214. Hooijmans, C.R., et al., Changes in cerebral blood volume and amyloid pathology in aged Alzheimer APP/PS1 mice on a docosahexaenoic acid (DHA) diet or cholesterol enriched Typical Western Diet (TWD). Neurobiol Dis, 2007. 28(1): p. 16-29.

215. Julien, C., et al., High-fat diet aggravates amyloid-beta and tau pathologies in the 3xTg-AD mouse model. Neurobiol Aging, 2010. 31(9): p. 1516-31.

216. Simons, M., et al., Cholesterol and Alzheimer's disease: is there a link? Neurology, 2001. 57(6): p. 1089-93.

217. Notkola, I.L., et al., Serum total cholesterol, apolipoprotein E epsilon 4 allele, and Alzheimer's disease. Neuroepidemiology, 1998. 17(1): p. 14-20.

218. Puglielli, L., R.E. Tanzi, and D.M. Kovacs, Alzheimer's disease: the cholesterol connection. Nat Neurosci, 2003. 6(4): p. 345-51.

219. Evans, R.M., et al., Cholesterol and APOE genotype interact to influence Alzheimer disease progression. Neurology, 2004. 62(10): p. 1869-71.

220. Kivipelto, M., et al., Apolipoprotein E epsilon4 allele, elevated midlife total cholesterol level, and high midlife systolic blood pressure are independent risk factors for late-life Alzheimer disease. Ann Intern Med, 2002. 137(3): p. 149-55.

221. Refolo, L.M., et al., Hypercholesterolemia accelerates the Alzheimer's amyloid pathology in a transgenic mouse model. Neurobiol Dis, 2000. 7(4): p. 321-31.

222. Refolo, L.M., et al., A cholesterol-lowering drug reduces beta-amyloid pathology in a transgenic mouse model of Alzheimer's disease. Neurobiol Dis, 2001. 8(5): p. 890-9.

223. Lim, G.P., et al., A diet enriched with the omega-3 fatty acid docosahexaenoic acid reduces amyloid burden in an aged Alzheimer mouse model. J Neurosci, 2005. 25(12): p. 3032-40.

224. Oksman, M., et al., Impact of different saturated fatty acid, polyunsaturated fatty acid and cholesterol containing diets on beta-amyloid accumulation in APP/PS1 transgenic mice. Neurobiol Dis, 2006. 23(3): p. 563-72.

225. Green, K.N., et al., Dietary docosahexaenoic acid and docosapentaenoic acid ameliorate amyloid-beta and tau pathology via a mechanism involving presenilin 1 levels. J Neurosci, 2007. 27(16): p. 4385-95.

226. Perez, S.E., et al., DHA diet reduces $A D$ pathology in young APPswe/PS1 Delta E9 transgenic mice: possible gender effects. J Neurosci Res, 2010. 88(5): p. 1026-40.

227. Morris, M.C., et al., Dietary fats and the risk of incident Alzheimer disease. Arch Neurol, 2003. 60(2): p. 194-200.

228. Morris, M.C., et al., Consumption of fish and $n-3$ fatty acids and risk of incident Alzheimer disease. Arch Neurol, 2003. 60(7): p. 940-6.

229. Morris, M.C., et al., Fish consumption and cognitive decline with age in a large community study. Arch Neurol, 2005. 62(12): p. 1849-53.

230. Hooijmans, C.R., et al., DHA and cholesterol containing diets influence Alzheimerlike pathology, cognition and cerebral vasculature in APPswe/PS1 $1 E 9$ mice. Neurobiol Dis, 2009. 33(3): p. 482-98. 
231. Arendash, G.W., et al., A diet high in omega-3 fatty acids does not improve or protect cognitive performance in Alzheimer's transgenic mice. Neuroscience, 2007. 149(2): p. 286-302.

232. Zhuo, J.M. and D. Pratico, Acceleration of brain amyloidosis in an Alzheimer's disease mouse model by a folate, vitamin B6 and B12-deficient diet. Exp Gerontol, 2010. 45(3): p. 195-201.

233. Fuso, A., et al., Changes in Presenilin 1 gene methylation pattern in diet-induced $B$ vitamin deficiency. Neurobiol Aging, 2009.

234. Nicolia, V., et al., B vitamin deficiency promotes tau phosphorylation through regulation of GSK3beta and PP2A. J Alzheimers Dis, 2010. 19(3): p. 895-907.

235. Kruman, II, et al., Folic acid deficiency and homocysteine impair DNA repair in hippocampal neurons and sensitize them to amyloid toxicity in experimental models of Alzheimer's disease. J Neurosci, 2002. 22(5): p. 1752-62.

236. Karuppagounder, S.S., et al., Thiamine deficiency induces oxidative stress and exacerbates the plaque pathology in Alzheimer's mouse model. Neurobiol Aging, 2009. 30(10): p. 1587-600.

237. Li, G., et al., Diet supplement CoQ10 delays brain atrophy in aged transgenic mice with mutations in the amyloid precursor protein: an in vivo volume MRI study. Biofactors, 2008. 32(1-4): p. 169-78.

238. Yang, X., et al., Coenzyme Q10 reduces beta-amyloid plaque in an APP/PS1 transgenic mouse model of Alzheimer's disease. J Mol Neurosci, 2010. 41(1): p. 110-3.

239. Ding, Y., et al., Retinoic acid attenuates beta-amyloid deposition and rescues memory deficits in an Alzheimer's disease transgenic mouse model. J Neurosci, 2008. 28(45): p. 11622-34.

240. Eskelinen, M.H. and M. Kivipelto, Caffeine as a protective factor in dementia and Alzheimer's disease. J Alzheimers Dis, 2010. 20 Suppl 1: p. S167-74.

241. Eskelinen, M.H., et al., Midlife coffee and tea drinking and the risk of late-life dementia: a population-based CAIDE study. J Alzheimers Dis, 2009. 16(1): p. 8591.

242. Santos, C., et al., Caffeine intake is associated with a lower risk of cognitive decline: a cohort study from Portugal. J Alzheimers Dis, 2010. 20 Suppl 1: p. S17585.

243. Santos, C., et al., Caffeine intake and dementia: systematic review and metaanalysis. J Alzheimers Dis, 2010. 20 Suppl 1: p. S187-204.

244. Arendash, G.W., et al., Caffeine reverses cognitive impairment and decreases brain amyloid-beta levels in aged Alzheimer's disease mice. J Alzheimers Dis, 2009. 17(3): p. 661-80.

245. Cao, C., et al., Caffeine suppresses amyloid-beta levels in plasma and brain of Alzheimer's disease transgenic mice. J Alzheimers Dis, 2009. 17(3): p. 681-97.

246. Obregon, D.F., et al., ADAM10 activation is required for green tea (-)epigallocatechin-3-gallate-induced alpha-secretase cleavage of amyloid precursor protein. J Biol Chem, 2006. 281(24): p. 16419-27.

247. Rezai-Zadeh, K., et al., Green tea epigallocatechin-3-gallate (EGCG) reduces betaamyloid mediated cognitive impairment and modulates tau pathology in Alzheimer transgenic mice. Brain Res, 2008. 1214: p. 177-87.

248. Rezai-Zadeh, K., et al., Green tea epigallocatechin-3-gallate (EGCG) modulates amyloid precursor protein cleavage and reduces cerebral amyloidosis in Alzheimer transgenic mice. J Neurosci, 2005. 25(38): p. 8807-14.

249. Rezai-Zadeh, K., et al., Flavonoid-mediated presenilin-1 phosphorylation reduces Alzheimer's disease beta-amyloid production. J Cell Mol Med, 2009. 13(3): p. 57488. 
250. Wang, J., et al., Moderate consumption of Cabernet Sauvignon attenuates Abeta neuropathology in a mouse model of Alzheimer's disease. FASEB J, 2006. 20(13): p. 2313-20.

251. Ho, L., et al., Heterogeneity in red wine polyphenolic contents differentially influences Alzheimer's disease-type neuropathology and cognitive deterioration. $\mathrm{J}$ Alzheimers Dis, 2009. 16(1): p. 59-72.

252. Almeida, O.P., et al., Smoking is associated with reduced cortical regional gray matter density in brain regions associated with incipient Alzheimer disease. Am J Geriatr Psychiatry, 2008. 16(1): p. 92-8.

253. Almeida, O.P., et al., Smoking as a risk factor for Alzheimer's disease: contrasting evidence from a systematic review of case-control and cohort studies. Addiction, 2002. 97(1): p. 15-28.

254. Cataldo, J.K., J.J. Prochaska, and S.A. Glantz, Cigarette smoking is a risk factor for Alzheimer's Disease: an analysis controlling for tobacco industry affiliation. J Alzheimers Dis, 2010. 19(2): p. 465-80.

255. Nordberg, A., et al., Chronic nicotine treatment reduces beta-amyloidosis in the brain of a mouse model of Alzheimer's disease (APPsw). J Neurochem, 2002. 81(3): p. 655-8.

256. Hellstrom-Lindahl, E., et al., Nicotine reduces $A$ beta in the brain and cerebral vessels of APPsw mice. Eur J Neurosci, 2004. 19(10): p. 2703-10.

257. Oddo, S., et al., Chronic nicotine administration exacerbates tau pathology in a transgenic model of Alzheimer's disease. Proc Natl Acad Sci U S A, 2005. 102(8): p. 3046-51.

258. Patel, N.V., et al., Caloric restriction attenuates Abeta-deposition in Alzheimer transgenic models. Neurobiol Aging, 2005. 26(7): p. 995-1000.

259. Colman, R.J., et al., Caloric restriction delays disease onset and mortality in rhesus monkeys. Science, 2009. 325(5937): p. 201-4.

260. Halagappa, V.K., et al., Intermittent fasting and caloric restriction ameliorate agerelated behavioral deficits in the triple-transgenic mouse model of Alzheimer's disease. Neurobiol Dis, 2007. 26(1): p. 212-20.

261. Lim, G.P., et al., Ibuprofen effects on Alzheimer pathology and open field activity in APPsw transgenic mice. Neurobiol Aging, 2001. 22(6): p. 983-91.

262. Lim, G.P., et al., The curry spice curcumin reduces oxidative damage and amyloid pathology in an Alzheimer transgenic mouse. J Neurosci, 2001. 21(21): p. 8370-7.

263. Joseph, J.A., et al., Blueberry supplementation enhances signaling and prevents behavioral deficits in an Alzheimer disease model. Nutr Neurosci, 2003. 6(3): p. 153-62.

264. Stackman, R.W., et al., Prevention of age-related spatial memory deficits in a transgenic mouse model of Alzheimer's disease by chronic Ginkgo biloba treatment. Exp Neurol, 2003. 184(1): p. 510-20.

265. Hardy, J., The amyloid hypothesis for Alzheimer's disease: a critical reappraisal. J Neurochem, 2009. 110(4): p. 1129-34.

266. Rutten, B.P., et al., Antioxidants and Alzheimer's disease: from bench to bedside (and back again). Curr Opin Clin Nutr Metab Care, 2002. 5(6): p. 645-51.

267. Rutten, B.P., et al., Age-related loss of synaptophysin immunoreactive presynaptic boutons within the hippocampus of APP751SL, PS1M146L, and APP751SL/PS1M146L transgenic mice. Am J Pathol, 2005. 167(1): p. 161-73.

268. Schmitz, C., et al., Hippocampal neuron loss exceeds amyloid plaque load in a transgenic mouse model of Alzheimer's disease. Am J Pathol, 2004. 164(4): p. 1495-502.

269. Dennissen, F.J., et al., Misframed proteins and neurodegeneration: a novel view on Alzheimer's and Parkinson's diseases. Neurodegener Dis, 2010. 7(1-3): p. 76-9. 
270. McNaull, B.B., et al., Inflammation and anti-inflammatory strategies for Alzheimer's disease--a mini-review. Gerontology, 2010. 56(1): p. 3-14.

271. van Leeuwen, F.W., et al., Frameshift mutants of beta amyloid precursor protein and ubiquitin-B in Alzheimer's and Down patients. Science, 1998. 279(5348): p. 242-7.

272. Brown, E.S., et al., The psychiatric sequelae of civilian trauma. Compr Psychiatry, 2000. 41(1): p. 19-23.

273. Rutten, B.P. and J. Mill, Epigenetic mediation of environmental influences in major psychotic disorders. Schizophr Bull, 2009. 35(6): p. 1045-56.

274. van Os, J. and J.P. Selten, Prenatal exposure to maternal stress and subsequent schizophrenia. The May 1940 invasion of The Netherlands. Br J Psychiatry, 1998. 172: p. 324-6.

275. Oddo, S., et al., Triple-transgenic model of Alzheimer's disease with plaques and tangles: intracellular Abeta and synaptic dysfunction. Neuron, 2003. 39(3): p. 40921.

276. Xu, P.T., et al., Human apolipoprotein E2, E3, and E4 isoform-specific transgenic mice: human-like pattern of glial and neuronal immunoreactivity in central nervous system not observed in wild-type mice. Neurobiol Dis, 1996. 3(3): p. 229-45.

277. Sturchler-Pierrat, C., et al., Two amyloid precursor protein transgenic mouse models with Alzheimer disease-like pathology. Proc Natl Acad Sci U S A, 1997. 94(24): p. 13287-92.

278. Blanchard, V., et al., Time sequence of maturation of dystrophic neurites associated with Abeta deposits in APP/PS1 transgenic mice. Exp Neurol, 2003. 184(1): p. 247-63.

279. Reaume, A.G., et al., Enhanced amyloidogenic processing of the beta-amyloid precursor protein in gene-targeted mice bearing the Swedish familial Alzheimer's disease mutations and a "humanized" Abeta sequence. J Biol Chem, 1996. 271(38): p. 23380-8.

280. Siman, R., et al., Presenilin-1 P264L knock-in mutation: differential effects on abeta production, amyloid deposition, and neuronal vulnerability. J Neurosci, 2000. 20(23): p. 8717-26.

281. Murphy, M.P., et al., Abeta solubility and deposition during AD progression and in APPxPS-1 knock-in mice. Neurobiol Dis, 2007. 27(3): p. 301-11.

282. Mucke, L., et al., High-level neuronal expression of abeta 1-42 in wild-type human amyloid protein precursor transgenic mice: synaptotoxicity without plaque formation. J Neurosci, 2000. 20(11): p. 4050-8.

283. Savonenko, A., et al., Episodic-like memory deficits in the APPswe/PS1 $E 9$ mouse model of Alzheimer's disease: relationships to beta-amyloid deposition and neurotransmitter abnormalities. Neurobiol Dis, 2005. 18(3): p. 602-17.

284. Holcomb, L., et al., Accelerated Alzheimer-type phenotype in transgenic mice carrying both mutant amyloid precursor protein and presenilin 1 transgenes. Nat Med, 1998. 4(1): p. 97-100.

285. Li, Q.X., et al., Intracellular accumulation of detergent-soluble amyloidogenic $A$ beta fragment of Alzheimer's disease precursor protein in the hippocampus of aged transgenic mice. J Neurochem, 1999. 72(6): p. 2479-87.

286. Buxbaum, J.D., et al., Expression of APP in brains of transgenic mice containing the entire human APP gene. Biochem Biophys Res Commun, 1993. 197(2): p. 63945.

287. Duyckaerts, C., M.C. Potier, and B. Delatour, Alzheimer disease models and human neuropathology: similarities and differences. Acta Neuropathol, 2008. 115(1): p. 5-38. 
288. Chui, D.H., et al., Transgenic mice with Alzheimer presenilin 1 mutations show accelerated neurodegeneration without amyloid plaque formation. Nat Med, 1999. 5(5): p. 560-4.

289. Howlett, D.R., et al., Cognitive correlates of Abeta deposition in male and female mice bearing amyloid precursor protein and presenilin-1 mutant transgenes. Brain Res, 2004. 1017(1-2): p. 130-6.

290. Gallant, M., et al., Focally elevated creatine detected in amyloid precursor protein (APP) transgenic mice and Alzheimer disease brain tissue. J Biol Chem, 2006. 281(1): p. 5-8.

291. Hsiao, K., et al., Correlative memory deficits, Abeta elevation, and amyloid plaques in transgenic mice. Science, 1996. 274(5284): p. 99-102.

292. Chishti, M.A., et al., Early-onset amyloid deposition and cognitive deficits in transgenic mice expressing a double mutant form of amyloid precursor protein 695. J Biol Chem, 2001. 276(24): p. 21562-70.

293. Tanemura, K., et al., Neurodegeneration with tau accumulation in a transgenic mouse expressing V337M human tau. J Neurosci, 2002. 22(1): p. 133-41.

294. Pardon, M.C., et al., Repeated novel cage exposure-induced improvement of early Alzheimer's-like cognitive and amyloid changes in TASTPM mice is unrelated to changes in brain endocannabinoids levels. Neurobiol Aging, 2009. 30(7): p. 1099113.

295. Levi, O., et al., Intraneuronal amyloid-beta plays a role in mediating the synergistic pathological effects of apoE4 and environmental stimulation. J Neurochem, 2007. 103(3): p. 1031-40.

296. Nichol, K., et al., Exercise improves cognition and hippocampal plasticity in APOE epsilon4 mice. Alzheimers Dement, 2009. 5(4): p. 287-94.

297. Mizoroki, T., et al., Aluminum induces tau aggregation in vitro but not in vivo. J Alzheimers Dis, 2007. 11(4): p. 419-27.

298. Nakagawa, Y., et al., Traumatic brain injury in young, amyloid-beta peptide overexpressing transgenic mice induces marked ipsilateral hippocampal atrophy and diminished Abeta deposition during aging. J Comp Neurol, 1999. 411(3): p. 390-8.

299. Nakagawa, Y., et al., Brain trauma in aged transgenic mice induces regression of established abeta deposits. Exp Neurol, 2000. 163(1): p. 244-52.

300. Arendash, G.W., et al., Caffeine protects Alzheimer's mice against cognitive impairment and reduces brain beta-amyloid production. Neuroscience, 2006. 142(4): p. 941-52.

301. Kessler, R.C., et al., The epidemiology of major depressive disorder: results from the National Comorbidity Survey Replication (NCS-R). JAMA, 2003. 289(23): p. 3095-105.

302. WHO-Europe. Mental health in the WHO European Region. 2003.

303. Austin, M.P., P. Mitchell, and G.M. Goodwin, Cognitive deficits in depression: possible implications for functional neuropathology. Br J Psychiatry, 2001. 178: $\mathrm{p}$. 200-6.

304. Castaneda, A.E., et al., A review on cognitive impairments in depressive and anxiety disorders with a focus on young adults. J Affect Disord, 2008. 106(1-2): p. $1-27$.

305. Hickie, I., et al., Reduced hippocampal volumes and memory loss in patients with early- and late-onset depression. Br J Psychiatry, 2005. 186: p. 197-202.

306. MacQueen, G.M., et al., Course of illness, hippocampal function, and hippocampal volume in major depression. Proc Natl Acad Sci U S A, 2003. 100(3): p. 1387-92.

307. Ravnkilde, B., et al., Cognitive deficits in major depression. Scand J Psychol, 2002. 43(3): p. 239-51. 
308. Sheline, Y.I., et al., Depression duration but not age predicts hippocampal volume loss in medically healthy women with recurrent major depression. J Neurosci, 1999. 19(12): p. 5034-43.

309. Bassuk, S.S., L.F. Berkman, and D. Wypij, Depressive symptomatology and incident cognitive decline in an elderly community sample. Arch Gen Psychiatry, 1998. 55(12): p. 1073-81.

310. Yaffe, K., et al., Depressive symptoms and cognitive decline in nondemented elderly women: a prospective study. Arch Gen Psychiatry, 1999. 56(5): p. 425-30.

311. Kelly, W.F., et al., Cushing's syndrome and depression--a prospective study of 26 patients. Br J Psychiatry, 1983. 142: p. 16-9.

312. Shibli-Rahhal, A., M. Van Beek, and J.A. Schlechte, Cushing's syndrome. Clin Dermatol, 2006. 24(4): p. 260-5.

313. Phelps, E.A., Human emotion and memory: interactions of the amygdala and hippocampal complex. Curr Opin Neurobiol, 2004. 14(2): p. 198-202.

314. Roozendaal, B., B.S. McEwen, and S. Chattarji, Stress, memory and the amygdala. Nat Rev Neurosci, 2009. 10(6): p. 423-33.

315. Arnsten, A.F., Stress signalling pathways that impair prefrontal cortex structure and function. Nat Rev Neurosci, 2009. 10(6): p. 410-22.

316. Hamilton, J.P., M. Siemer, and I.H. Gotlib, Amygdala volume in major depressive disorder: a meta-analysis of magnetic resonance imaging studies. Mol Psychiatry, 2008. 13(11): p. 993-1000.

317. Konarski, J.Z., et al., Volumetric neuroimaging investigations in mood disorders: bipolar disorder versus major depressive disorder. Bipolar Disord, 2008. 10(1): p. 137.

318. Aihara, M., et al., HPA axis dysfunction in unmedicated major depressive disorder and its normalization by pharmacotherapy correlates with alteration of neural activity in prefrontal cortex and limbic/paralimbic regions. Psychiatry Res, 2007. 155(3): p. 245-56.

319. Bell-McGinty, S., et al., Brain morphometric abnormalities in geriatric depression: long-term neurobiological effects of illness duration. Am J Psychiatry, 2002. 159(8): p. 1424-7.

320. Bremner, J.D., et al., Hippocampal volume reduction in major depression. Am J Psychiatry, 2000. 157(1): p. 115-8.

321. Janssen, J., et al., Hippocampal changes and white matter lesions in early-onset depression. Biol Psychiatry, 2004. 56(11): p. 825-31.

322. Sheline, Y.I., M.H. Gado, and H.C. Kraemer, Untreated depression and hippocampal volume loss. Am J Psychiatry, 2003. 160(8): p. 1516-8.

323. Sheline, Y.I., et al., Hippocampal atrophy in recurrent major depression. Proc Natl Acad Sci U S A, 1996. 93(9): p. 3908-13.

324. Colla, M., et al., Hippocampal volume reduction and HPA-system activity in major depression. J Psychiatr Res, 2007. 41(7): p. 553-60.

325. Frodl, T., et al., Hippocampal changes in patients with a first episode of major depression. Am J Psychiatry, 2002. 159(7): p. 1112-8.

326. Stockmeier, C.A., et al., Cellular changes in the postmortem hippocampus in major depression. Biol Psychiatry, 2004. 56(9): p. 640-50.

327. Aggleton, J.P., A description of the amygdalo-hippocampal interconnections in the macaque monkey. Exp Brain Res, 1986. 64(3): p. 515-26.

328. Pikkarainen, M., et al., Projections from the lateral, basal, and accessory basal nuclei of the amygdala to the hippocampal formation in rat. J Comp Neurol, 1999. 403(2): p. 229-60.

329. Richter-Levin, G., The amygdala, the hippocampus, and emotional modulation of memory. Neuroscientist, 2004. 10(1): p. 31-9. 
330. Kronenberg, G., et al., Reduced amygdala volume in newly admitted psychiatric inpatients with unipolar major depression. J Psychiatr Res, 2009.

331. van Eijndhoven, P., et al., Amygdala volume marks the acute state in the early course of depression. Biol Psychiatry, 2009. 65(9): p. 812-8.

332. Abercrombie, H.C., et al., Metabolic rate in the right amygdala predicts negative affect in depressed patients. Neuroreport, 1998. 9(14): p. 3301-7.

333. Drevets, W.C., et al., Glucose metabolism in the amygdala in depression: relationship to diagnostic subtype and plasma cortisol levels. Pharmacol Biochem Behav, 2002. 71(3): p. 431-47.

334. Drevets, W.C., et al., A functional anatomical study of unipolar depression. J Neurosci, 1992. 12(9): p. 3628-41.

335. Perico, C.A., et al., Relationship between regional cerebral blood flow and separate symptom clusters of major depression: a single photon emission computed tomography study using statistical parametric mapping. Neurosci Lett, 2005. 384(3): p. 265-70.

336. Maletic, V., et al., Neurobiology of depression: an integrated view of key findings. Int J Clin Pract, 2007. 61(12): p. 2030-40.

337. Zald, D.H., Orbital versus dorsolateral prefrontal cortex: anatomical insights into content versus process differentiation models of the prefrontal cortex. Ann $\mathrm{N} \mathrm{Y}$ Acad Sci, 2007. 1121: p. 395-406.

338. Koenigs, M. and J. Grafman, The functional neuroanatomy of depression: distinct roles for ventromedial and dorsolateral prefrontal cortex. Behav Brain Res, 2009. 201(2): p. 239-43.

339. Wall, P.M. and C. Messier, The hippocampal formation--orbitomedial prefrontal cortex circuit in the attentional control of active memory. Behav Brain Res, 2001. 127(1-2): p. 99-117.

340. Biver, F., et al., Frontal and parietal metabolic disturbances in unipolar depression. Biol Psychiatry, 1994. 36(6): p. 381-8.

341. Galynker, II, et al., Hypofrontality and negative symptoms in major depressive disorder. J Nucl Med, 1998. 39(4): p. 608-12.

342. Bremner, J.D., et al., Reduced volume of orbitofrontal cortex in major depression. Biol Psychiatry, 2002. 51(4): p. 273-9.

343. Praag, H.M.v., J.v. Os, and E.R.d. Kloet, Stress, the brain and depression. 2004, Cambridge, N.Y.: Cambridge University Press. XII, $283 \mathrm{s.}$

344. Joels, M., Corticosteroid effects in the brain: U-shape it. Trends Pharmacol Sci, 2006. 27(5): p. 244-50.

345. Harfstrand, A., et al., Glucocorticoid receptor immunoreactivity in monoaminergic neurons of rat brain. Proc Natl Acad Sci U S A, 1986. 83(24): p. 9779-83.

346. Reul, J.M. and E.R. de Kloet, Anatomical resolution of two types of corticosterone receptor sites in rat brain with in vitro autoradiography and computerized image analysis. J Steroid Biochem, 1986. 24(1): p. 269-72.

347. Carroll, B.J., et al., Urinary free cortisol excretion in depression. Psychol Med, 1976. 6(1): p. 43-50.

348. Carroll, B.J., G.C. Curtis, and J. Mendels, Cerebrospinal fluid and plasma free cortisol concentrations in depression. Psychol Med, 1976. 6(2): p. 235-44.

349. Gold, P.W., et al., Abnormal ACTH and cortisol responses to ovine corticotropin releasing factor in patients with primary affective disorder. Prog Neuropsychopharmacol Biol Psychiatry, 1986. 10(1): p. 57-65.

350. Juruena, M.F., et al., Different responses to dexamethasone and prednisolone in the same depressed patients. Psychopharmacology (Berl), 2006. 189(2): p. 225-35.

351. Pfohl, B., et al., Differences in plasma ACTH and cortisol between depressed patients and normal controls. Biol Psychiatry, 1985. 20(10): p. 1055-72. 
352. Kunugi, $\mathrm{H}$., et al., Assessment of the dexamethasone/CRH test as a statedependent marker for hypothalamic-pituitary-adrenal (HPA) axis abnormalities in major depressive episode: a Multicenter Study. Neuropsychopharmacology, 2006. 31(1): p. 212-20.

353. Kalin, N.H., S.J. Weiler, and S.E. Shelton, Plasma ACTH and cortisol concentrations before and after dexamethasone. Psychiatry Res, 1982. 7(1): p. 8792.

354. Roy, A., et al., Pre- and post-dexamethasone plasma ACTH levels in depressed patients and normal controls. J Affect Disord, 1986. 10(2): p. 95-9.

355. Posener, J.A., et al., 24-Hour monitoring of cortisol and corticotropin secretion in psychotic and nonpsychotic major depression. Arch Gen Psychiatry, 2000. 57(8): p. 755-60.

356. Heuser, I., A. Yassouridis, and F. Holsboer, The combined dexamethasone/CRH test: a refined laboratory test for psychiatric disorders. J Psychiatr Res, 1994. 28(4): p. 341-56.

357. Bardeleben, U. and F. Holsboer, Cortisol response to a combined dexamethasonehuman corticotrophin-releasing hormone challenge in patients with depression. J Neuroendocrinol, 1989. 1(6): p. 485-8.

358. Holsboer-Trachsler, E., R. Stohler, and M. Hatzinger, Repeated administration of the combined dexamethasone-human corticotropin releasing hormone stimulation test during treatment of depression. Psychiatry Res, 1991. 38(2): p. 163-71.

359. Banki, C.M., et al., CSF corticotropin-releasing factor-like immunoreactivity in depression and schizophrenia. Am J Psychiatry, 1987. 144(7): p. 873-7.

360. Nemeroff, C.B., et al., Elevated concentrations of CSF corticotropin-releasing factor-like immunoreactivity in depressed patients. Science, 1984. 226(4680): p. 1342-4.

361. Risch, S.C., et al., Limbic-hypothalamic-pituitary-adrenal axis activity and ventricular-to-brain ratio studies in affective illness and schizophrenia. Neuropsychopharmacology, 1992. 6(2): p. 95-100.

362. Raadsheer, F.C., et al., Corticotropin-releasing hormone mRNA levels in the paraventricular nucleus of patients with Alzheimer's disease and depression. Am J Psychiatry, 1995. 152(9): p. 1372-6.

363. Nemeroff, C.B., et al., Reduced corticotropin releasing factor binding sites in the frontal cortex of suicide victims. Arch Gen Psychiatry, 1988. 45(6): p. 577-9.

364. Sapolsky, R.M., L.C. Krey, and B.S. McEwen, The neuroendocrinology of stress and aging: the glucocorticoid cascade hypothesis. Endocr Rev, 1986. 7(3): p. 284301.

365. Pariante, C.M., Glucocorticoid receptor function in vitro in patients with major depression. Stress, 2004. 7(4): p. 209-19.

366. Gerges, N.Z., J.L. Stringer, and K.A. Alkadhi, Combination of hypothyroidism and stress abolishes early LTP in the CA1 but not dentate gyrus of hippocampus of adult rats. Brain Res, 2001. 922(2): p. 250-60.

367. Pavlides, C., L.G. Nivon, and B.S. McEwen, Effects of chronic stress on hippocampal long-term potentiation. Hippocampus, 2002. 12(2): p. 245-57.

368. Van Londen, L., et al., Neuropsychological performance and plasma cortisol, arginine vasopressin and oxytocin in patients with major depression. Psychol Med, 1998. 28(2): p. 275-84.

369. Kirschbaum, C., et al., Stress- and treatment-induced elevations of cortisol levels associated with impaired declarative memory in healthy adults. Life Sci, 1996. 58(17): p. 1475-83.

370. Lupien, S., et al., Basal cortisol levels and cognitive deficits in human aging. J Neurosci, 1994. 14(5 Pt 1): p. 2893-903. 
371. Lupien, S.J., et al., Stress hormones and human memory function across the lifespan. Psychoneuroendocrinology, 2005. 30(3): p. 225-42.

372. Honkaniemi, J., et al., Colocalization of peptide and glucocorticoid receptor immunoreactivities in rat central amygdaloid nucleus. Neuroendocrinology, 1992. 55(4): p. 451-9.

373. Meaney, M.J. and D.H. Aitken, [3H]Dexamethasone binding in rat frontal cortex. Brain Res, 1985. 328(1): p. 176-80.

374. Young, A.H., et al., The effects of chronic administration of hydrocortisone on cognitive function in normal male volunteers. Psychopharmacology (Berl), 1999. 145(3): p. 260-6.

375. Prickaerts, J. and T. Steckler, Effects of glucocorticoids on emotion and cognitive processes in animals, in Handbook of Stress and the Brain, N.H.K.a.J.M.H.M.R. T. Steckler, Editor. 2005, Elsevier B.V. p. 359-385.

376. Buchanan, T.W. and W.R. Lovallo, Enhanced memory for emotional material following stress-level cortisol treatment in humans. Psychoneuroendocrinology, 2001. 26(3): p. 307-17.

377. Rimmele, U., et al., Cortisol has different effects on human memory for emotional and neutral stimuli. Neuroreport, 2003. 14(18): p. 2485-8.

378. Maheu, F.S., R. Joober, and S.J. Lupien, Declarative memory after stress in humans: differential involvement of the beta-adrenergic and corticosteroid systems. $\mathrm{J}$ Clin Endocrinol Metab, 2005. 90(3): p. 1697-704.

379. Jacobs, B.L. and E.C. Azmitia, Structure and function of the brain serotonin system. Physiol Rev, 1992. 72(1): p. 165-229.

380. Steinbusch, H., Distribution of serotonin-immunoreactive neurons and their projections in the central nervous system of the rat, in Classical Transmitters and Transmitter Receptors in the CNS, part II, The Handbook of Chemical Neuroanatomy, A. Bjorklund, T. Hokfelt, and M.J. Kuhar, Editors. 1984, Elsevier: Amsterdam. p. 68-125.

381. Steinbusch, H.W., Distribution of serotonin-immunoreactivity in the central nervous system of the rat-cell bodies and terminals. Neuroscience, 1981. 6(4): p. 557-618.

382. Lanfumey, L., et al., Corticosteroid-serotonin interactions in the neurobiological mechanisms of stress-related disorders. Neurosci Biobehav Rev, 2008. 32(6): p. 1174-84.

383. Meltzer, C.C., et al., Serotonin in aging, late-life depression, and Alzheimer's disease: the emerging role of functional imaging. Neuropsychopharmacology, 1998. 18(6): p. 407-30.

384. Gaspar, P., O. Cases, and L. Maroteaux, The developmental role of serotonin: news from mouse molecular genetics. Nat Rev Neurosci, 2003. 4(12): p. 1002-12.

385. Burnet, P.W., et al., The distribution of 5-HT1A and 5-HT2A receptor mRNA in human brain. Brain Res, 1995. 676(1): p. 157-68.

386. DeFelipe, J., et al., Pyramidal cell axons show a local specialization for GABA and 5-HT inputs in monkey and human cerebral cortex. J Comp Neurol, 2001. 433(1): p. $148-55$.

387. Martin, G.R. and P.P. Humphrey, Receptors for 5-hydroxytryptamine: current perspectives on classification and nomenclature. Neuropharmacology, 1994. 33(34): p. 261-73.

388. Buhot, M.C., S. Martin, and L. Segu, Role of serotonin in memory impairment. Ann Med, 2000. 32(3): p. 210-21.

389. Drevets, W.C., et al., PET imaging of serotonin $1 \mathrm{~A}$ receptor binding in depression. Biol Psychiatry, 1999. 46(10): p. 1375-87.

390. Sargent, P.A., et al., Brain serotonin1A receptor binding measured by positron emission tomography with [11C]WAY-100635: effects of depression and antidepressant treatment. Arch Gen Psychiatry, 2000. 57(2): p. 174-80. 
391. Lopez-Figueroa, A.L., et al., Serotonin 5-HT1A, 5-HT1B, and 5-HT2A receptor mRNA expression in subjects with major depression, bipolar disorder, and schizophrenia. Biol Psychiatry, 2004. 55(3): p. 225-33.

392. Arango, V., et al., Serotonin $1 A$ receptors, serotonin transporter binding and serotonin transporter mRNA expression in the brainstem of depressed suicide victims. Neuropsychopharmacology, 2001. 25(6): p. 892-903.

393. Boldrini, M., et al., Serotonin-1A autoreceptor binding in the dorsal raphe nucleus of depressed suicides. J Psychiatr Res, 2008. 42(6): p. 433-42.

394. Lopez, J.F., et al., A.E. Bennett Research Award. Regulation of serotonin1A, glucocorticoid, and mineralocorticoid receptor in rat and human hippocampus: implications for the neurobiology of depression. Biol Psychiatry, 1998. 43(8): p. 547-73.

395. Arango, V., et al., Localized alterations in pre- and postsynaptic serotonin binding sites in the ventrolateral prefrontal cortex of suicide victims. Brain Res, 1995. 688(1-2): p. 121-33.

396. Stockmeier, C.A., et al., Increase in serotonin-1A autoreceptors in the midbrain of suicide victims with major depression-postmortem evidence for decreased serotonin activity. J Neurosci, 1998. 18(18): p. 7394-401.

397. Hensler, J.G. and K.A. Truett, Effect of chronic serotonin-2 receptor agonist or antagonist administration on serotonin-1A receptor sensitivity. Neuropsychopharmacology, 1998. 19(5): p. 354-64.

398. Tecott, L.H., et al., Perturbed dentate gyrus function in serotonin 5-HT2C receptor mutant mice. Proc Natl Acad Sci U S A, 1998. 95(25): p. 15026-31.

399. Arora, R.C. and H.Y. Meltzer, Increased serotonin2 (5-HT2) receptor binding as measured by $3 H$-lysergic acid diethylamide (3H-LSD) in the blood platelets of depressed patients. Life Sci, 1989. 44(11): p. 725-34.

400. Arora, R.C. and H.Y. Meltzer, Serotonergic measures in the brains of suicide victims: 5-HT2 binding sites in the frontal cortex of suicide victims and control subjects. Am J Psychiatry, 1989. 146(6): p. 730-6.

401. Mann, J.J., et al., Increased serotonin2 and beta-adrenergic receptor binding in the frontal cortices of suicide victims. Arch Gen Psychiatry, 1986. 43(10): p. 954-9.

402. Stanley, M. and J.J. Mann, Increased serotonin-2 binding sites in frontal cortex of suicide victims. Lancet, 1983. 1(8318): p. 214-6.

403. Yates, M., et al., 5HT2 receptor changes in major depression. Biol Psychiatry, 1990. 27(5): p. 489-96.

404. Yatham, L.N., et al., Brain serotonin2 receptors in major depression: a positron emission tomography study. Arch Gen Psychiatry, 2000. 57(9): p. 850-8.

405. Santarelli, L., et al., Requirement of hippocampal neurogenesis for the behavioral effects of antidepressants. Science, 2003. 301(5634): p. 805-9.

406. Duman, R.S. and L.M. Monteggia, A neurotrophic model for stress-related mood disorders. Biol Psychiatry, 2006. 59(12): p. 1116-27.

407. Mattson, M.P., S. Maudsley, and B. Martin, BDNF and 5-HT: a dynamic duo in agerelated neuronal plasticity and neurodegenerative disorders. Trends Neurosci, 2004. 27(10): p. 589-94.

408. Mamounas, L.A., et al., Brain-derived neurotrophic factor promotes the survival and sprouting of serotonergic axons in rat brain. J Neurosci, 1995. 15(12): p. 7929-39.

409. Karege, F., et al., Neurotrophin levels in postmortem brains of suicide victims and the effects of antemortem diagnosis and psychotropic drugs. Brain Res Mol Brain Res, 2005. 136(1-2): p. 29-37.

410. Smith, M.A., et al., Stress and glucocorticoids affect the expression of brain-derived neurotrophic factor and neurotrophin-3 mRNAs in the hippocampus. J Neurosci, 1995. 15(3 Pt 1): p. 1768-77. 
411. $\mathrm{Xu}, \mathrm{H}$., et al., Synergetic effects of quetiapine and venlafaxine in preventing the chronic restraint stress-induced decrease in cell proliferation and BDNF expression in rat hippocampus. Hippocampus, 2006. 16(6): p. 551-9.

412. Frodl, T., et al., Association of the brain-derived neurotrophic factor Val66Met polymorphism with reduced hippocampal volumes in major depression. Arch Gen Psychiatry, 2007. 64(4): p. 410-6.

413. Hwang, J.P., et al., The Val66Met polymorphism of the brain-derived neurotrophicfactor gene is associated with geriatric depression. Neurobiol Aging, 2006. 27(12): p. 1834-7.

414. Lesch, K.P., et al., Association of anxiety-related traits with a polymorphism in the serotonin transporter gene regulatory region. Science, 1996. 274(5292): p. 152731.

415. Lesch, K.P., et al., Mosaicism for a serotonin transporter gene promoter-associated deletion: decreased recombination in depression. J Neural Transm, 1999. 106(1112): p. 1223-30.

416. Caspi, A., et al., Influence of life stress on depression: moderation by a polymorphism in the 5-HTT gene. Science, 2003. 301(5631): p. 386-9.

417. O'Hara, R., et al., Serotonin transporter polymorphism, memory and hippocampal volume in the elderly: association and interaction with cortisol. Mol Psychiatry, 2007. 12(6): p. 544-55.

418. Frodl, T., et al., Reduced hippocampal volumes associated with the long variant of the serotonin transporter polymorphism in major depression. Arch Gen Psychiatry, 2004. 61(2): p. 177-83.

419. Hariri, A.R., et al., Serotonin transporter genetic variation and the response of the human amygdala. Science, 2002. 297(5580): p. 400-3.

420. Canli, T., et al., Beyond affect: a role for genetic variation of the serotonin transporter in neural activation during a cognitive attention task. Proc Natl Acad Sci U S A, 2005. 102(34): p. 12224-9.

421. Sakanaka, M., T. Shibasaki, and K. Lederis, Corticotropin releasing factor-like immunoreactivity in the rat brain as revealed by a modified cobalt-glucose oxidasediaminobenzidine method. J Comp Neurol, 1987. 260(2): p. 256-98.

422. Valentino, R.J., L. Liouterman, and E.J. Van Bockstaele, Evidence for regional heterogeneity in corticotropin-releasing factor interactions in the dorsal raphe nucleus. J Comp Neurol, 2001. 435(4): p. 450-63.

423. Austin, M.C., J.E. Janosky, and H.A. Murphy, Increased corticotropin-releasing hormone immunoreactivity in monoamine-containing pontine nuclei of depressed suicide men. Mol Psychiatry, 2003. 8(3): p. 324-32.

424. Lowry, C.A., et al., Corticotropin-releasing factor increases in vitro firing rates of serotonergic neurons in the rat dorsal raphe nucleus: evidence for activation of a topographically organized mesolimbocortical serotonergic system. J Neurosci, 2000. 20(20): p. 7728-36.

425. Mo, B., et al., Restraint stress increases serotonin release in the central nucleus of the amygdala via activation of corticotropin-releasing factor receptors. Brain Res Bull, 2008. 76(5): p. 493-8.

426. Bagdy, G., et al., Long-term cortisol treatment impairs behavioral and neuroendocrine responses to 5-HT1 agonists in the rat. Neuroendocrinology, 1989. 50(3): p. 241-7.

427. Morley-Fletcher, S., et al., Chronic treatment with imipramine reverses immobility behaviour, hippocampal corticosteroid receptors and cortical 5-HT(1A) receptor $m R N A$ in prenatally stressed rats. Neuropharmacology, 2004. 47(6): p. 841-7.

428. De Kloet, E.R., H. Sybesma, and H.M. Reul, Selective control by corticosterone of serotonin1 receptor capacity in raphe-hippocampal system. Neuroendocrinology, 1986. 42(6): p. 513-21. 
429. Mendelson, S.D. and B.S. McEwen, Autoradiographic analyses of the effects of adrenalectomy and corticosterone on 5-HT1A and 5-HT1B receptors in the dorsal hippocampus and cortex of the rat. Neuroendocrinology, 1992. 55(4): p. 444-50.

430. Zhong, P. and R.D. Ciaranello, Transcriptional regulation of hippocampal 5-HT1a receptors by corticosteroid hormones. Brain Res Mol Brain Res, 1995. 29(1): p. 2334.

431. De Kloet, E.R., et al., Decreased serotonin turnover in the dorsal hippocampus of rat brain shortly after adrenalectomy: selective normalization after corticosterone substitution. Brain Res, 1982. 239(2): p. 659-63.

432. Van Loon, G.R., A. Shum, and M.J. Sole, Decreased brain serotonin turnover after short term (two-hour) adrenalectomy in rats: a comparison of four turnover methods. Endocrinology, 1981. 108(4): p. 1392-402.

433. Maes, M., et al., Suppressant effects of dexamethasone on the availability of plasma L-tryptophan and tyrosine in healthy controls and in depressed patients. Acta Psychiatr Scand, 1990. 81(1): p. 19-23.

434. Maes, M., et al., Effects of dexamethasone on the availability of L-tryptophan and on the insulin and FFA concentrations in unipolar depressed patients. Biol Psychiatry, 1990. 27(8): p. 854-62.

435. Singh, V.B., et al., Increase in cortical and midbrain tryptophan hydroxylase activity by intracerebroventricular administration of corticotropin releasing factor: block by adrenalectomy, by RU 38486 and by bilateral lesions to the central nucleus of the amygdala. Neurochem Int, 1992. 20(1): p. 81-92.

436. Fuller, R.W. and H.D. Snoddy, Serotonin receptor subtypes involved in the elevation of serum corticosterone concentration in rats by direct- and indirect-acting serotonin agonists. Neuroendocrinology, 1990. 52(2): p. 206-11.

437. Lesch, K.P., et al., 5-HT1A receptor responsivity in unipolar depression. Evaluation of ipsapirone-induced ACTH and cortisol secretion in patients and controls. Biol Psychiatry, 1990. 28(7): p. 620-8.

438. Feldman, S. and J. Weidenfeld, Depletion of hypothalamic norepinephrine and serotonin enhances the dexamethasone negative feedback effect on adrenocortical secretion. Psychoneuroendocrinology, 1991. 16(5): p. 397-405.

439. Michelsen, K.A., J. Prickaerts, and H.W. Steinbusch, The dorsal raphe nucleus and serotonin: implications for neuroplasticity linked to major depression and Alzheimer's disease. Prog Brain Res, 2008. 172: p. 233-64.

440. Michelsen, K.A., C. Schmitz, and H.W. Steinbusch, The dorsal raphe nucleus--from silver stainings to a role in depression. Brain Res Rev, 2007. 55(2): p. 329-42.

441. Liposits, Z., C. Phelix, and W.K. Paull, Synaptic interaction of serotonergic axons and corticotropin releasing factor (CRF) synthesizing neurons in the hypothalamic paraventricular nucleus of the rat. $A$ light and electron microscopic immunocytochemical study. Histochemistry, 1987. 86(6): p. 541-9.

442. Zhang, Y., et al., Desensitization of 5-HT1A receptors by 5-HT2A receptors in neuroendocrine neurons in vivo. J Pharmacol Exp Ther, 2004. 310(1): p. 59-66.

443. Osei-Owusu, P., et al., 5-Hydroxytryptamine 1A receptors in the paraventricular nucleus of the hypothalamus mediate oxytocin and adrenocorticotropin hormone release and some behavioral components of the serotonin syndrome. J Pharmacol Exp Ther, 2005. 313(3): p. 1324-30.

444. Seckl, J.R. and G. Fink, Use of in situ hybridization to investigate the regulation of hippocampal corticosteroid receptors by monoamines. J Steroid Biochem Mol Biol, 1991. 40(4-6): p. 685-8.

445. Andrade, R. and R.A. Nicoll, Pharmacologically distinct actions of serotonin on single pyramidal neurones of the rat hippocampus recorded in vitro. J Physiol, 1987. 394: p. 99-124. 
446. Beck, S.G., et al., Corticosterone alters 5-HT1A receptor-mediated hyperpolarization in area CA1 hippocampal pyramidal neurons. Neuropsychopharmacology, 1996. 14(1): p. 27-33.

447. Goodyer, I.M., et al., Serotonin transporter genotype, morning cortisol and subsequent depression in adolescents. Br J Psychiatry, 2009. 195(1): p. 39-45.

448. Alexander, N., et al., Gene-environment interactions predict cortisol responses after acute stress: Implications for the etiology of depression. Psychoneuroendocrinology, 2009.

449. Gotlib, I.H., et al., HPA axis reactivity: a mechanism underlying the associations among 5-HTTLPR, stress, and depression. Biol Psychiatry, 2008. 63(9): p. 847-51.

450. Derijk, R.H. and E.R. de Kloet, Corticosteroid receptor polymorphisms: determinants of vulnerability and resilience. Eur J Pharmacol, 2008. 583(2-3): p. 303-11.

451. Bet, P.M., et al., Glucocorticoid receptor gene polymorphisms and childhood adversity are associated with depression: New evidence for a gene-environment interaction. Am J Med Genet B Neuropsychiatr Genet, 2009. 150B(5): p. 660-9.

452. Bierman, E.J., et al., Effects of anxiety versus depression on cognition in later life. Am J Geriatr Psychiatry, 2005. 13(8): p. 686-93.

453. Chodosh, J., et al., Depressive symptoms as a predictor of cognitive decline: MacArthur Studies of Successful Aging. Am J Geriatr Psychiatry, 2007. 15(5): p. 406-15.

454. Raji, M.A., et al., Depressive symptoms and cognitive change in older Mexican Americans. J Geriatr Psychiatry Neurol, 2007. 20(3): p. 145-52.

455. Wilson, R.S., et al., Depressive symptoms and cognitive decline in a community population of older persons. J Neurol Neurosurg Psychiatry, 2004. 75(1): p. 126-9.

456. Fernandez Martinez, M., et al., Risk factors for dementia in the epidemiological study of Munguialde County (Basque Country-Spain). BMC Neurol, 2008. 8: p. 39.

457. Kessing, L.V. and P.K. Andersen, Does the risk of developing dementia increase with the number of episodes in patients with depressive disorder and in patients with bipolar disorder? J Neurol Neurosurg Psychiatry, 2004. 75(12): p. 1662-6.

458. Modrego, P.J. and J. Ferrandez, Depression in patients with mild cognitive impairment increases the risk of developing dementia of Alzheimer type: a prospective cohort study. Arch Neurol, 2004. 61(8): p. 1290-3.

459. Morgan, M.D., et al., Rates of depression in individuals with pathologic but not clinical Alzheimer disease are lower than those in individuals without the disease: findings from the Baltimore Longitudinal Study on Aging (BLSA). Alzheimer Dis Assoc Disord, 2007. 21(3): p. 199-204.

460. Ownby, R.L., et al., Depression and risk for Alzheimer disease: systematic review, meta-analysis, and metaregression analysis. Arch Gen Psychiatry, 2006. 63(5): p. 530-8.

461. Kessing, L.V. and F.M. Nilsson, Increased risk of developing dementia in patients with major affective disorders compared to patients with other medical illnesses. J Affect Disord, 2003. 73(3): p. 261-9.

462. Laske, C., et al., Inverse association of cortisol serum levels with T-tau, P-tau 181 and P-tau 231 peptide levels and T-tau/Abeta 1-42 ratios in CSF in patients with mild Alzheimer's disease dementia. Eur Arch Psychiatry Clin Neurosci, 2009. 259(2): p. 80-5.

463. Elgh, E., et al., Cognitive dysfunction, hippocampal atrophy and glucocorticoid feedback in Alzheimer's disease. Biol Psychiatry, 2006. 59(2): p. 155-61.

464. Catania, C., et al., The amyloidogenic potential and behavioral correlates of stress. Mol Psychiatry, 2009. 14(1): p. 95-105. 
465. Sotiropoulos, I., et al., Glucocorticoids trigger Alzheimer disease-like pathobiochemistry in rat neuronal cells expressing human tau. J Neurochem, 2008. 107(2): p. 385-97.

466. Abraham, I., et al., Chronic corticosterone administration dose-dependently modulates Abeta(1-42)- and NMDA-induced neurodegeneration in rat magnocellular nucleus basalis. J Neuroendocrinol, 2000. 12(6): p. 486-94.

467. Yao, Y.Y., et al., Memory and learning impairment induced by dexamethasone in senescent but not young mice. Eur J Pharmacol, 2007. 574(1): p. 20-8.

468. Rissman, R.A., et al., Corticotropin-releasing factor receptors differentially regulate stress-induced tau phosphorylation. J Neurosci, 2007. 27(24): p. 6552-62.

469. Curcio, C.A. and T. Kemper, Nucleus raphe dorsalis in dementia of the Alzheimer type: neurofibrillary changes and neuronal packing density. J Neuropathol Exp Neurol, 1984. 43(4): p. 359-68.

470. Hendricksen, M., et al., Neuropathological study of the dorsal raphe nuclei in latelife depression and Alzheimer's disease with and without depression. Am J Psychiatry, 2004. 161(6): p. 1096-102.

471. Lyness, S.A., C. Zarow, and H.C. Chui, Neuron loss in key cholinergic and aminergic nuclei in Alzheimer disease: a meta-analysis. Neurobiol Aging, 2003. 24(1): p. 1-23.

472. Yamamoto, T. and A. Hirano, Nucleus raphe dorsalis in Alzheimer's disease: neurofibrillary tangles and loss of large neurons. Ann Neurol, 1985. 17(6): p. 573-7.

473. Palmer, A.M., et al., Presynaptic serotonergic dysfunction in patients with Alzheimer's disease. J Neurochem, 1987. 48(1): p. 8-15.

474. $\mathrm{Li}, \mathrm{T}$., et al., Allelic functional variation of serotonin transporter expression is a susceptibility factor for late onset Alzheimer's disease. Neuroreport, 1997. 8(3): p. 683-6.

475. Mossner, R., et al., The serotonin transporter in Alzheimer's and Parkinson's disease. J Neural Transm Suppl, 2000(60): p. 345-50.

476. Arai, H., K. Kosaka, and R. lizuka, Changes of biogenic amines and their metabolites in postmortem brains from patients with Alzheimer-type dementia. J Neurochem, 1984. 43(2): p. 388-93.

477. Bowen, D.M., et al., Biochemical assessment of serotonergic and cholinergic dysfunction and cerebral atrophy in Alzheimer's disease. J Neurochem, 1983. 41(1): p. 266-72.

478. Cross, A.J., et al., Serotonin receptor changes in dementia of the Alzheimer type. J Neurochem, 1984. 43(6): p. 1574-81.

479. Crow, T.J., et al., Neurotransmitter receptors and monoamine metabolites in the brains of patients with Alzheimer-type dementia and depression, and suicides. Neuropharmacology, 1984. 23(12B): p. 1561-9.

480. Jansen, K.L., et al., Alzheimer's disease: changes in hippocampal N-methyl-Daspartate, quisqualate, neurotensin, adenosine, benzodiazepine, serotonin and opioid receptors--an autoradiographic study. Neuroscience, 1990. 39(3): p. 613-27.

481. Middlemiss, D.N., et al., Binding of the novel serotonin agonist 8-hydroxy-2-(di-npropylamino) tetralin in normal and Alzheimer brain. J Neurochem, 1986. 46(3): p. 993-6.

482. Palmer, A.M., et al., Possible neurotransmitter basis of behavioral changes in Alzheimer's disease. Ann Neurol, 1988. 23(6): p. 616-20.

483. Perry, E.K., et al., Cortical serotonin-S2 receptor binding abnormalities in patients with Alzheimer's disease: comparisons with Parkinson's disease. Neurosci Lett, 1984. 51(3): p. 353-7.

484. Reynolds, G.P., et al., Reduced binding of [3H]ketanserin to cortical 5-ht2 receptors in senile dementia of the Alzheimer type. Neurosci Lett, 1984. 44(1): p. 47-51. 
485. Truchot, L., et al., A distinct [18F]MPPF PET profile in amnestic mild cognitive impairment compared to mild Alzheimer's disease. Neuroimage, 2008. 40(3): p. 1251-6.

486. Chen, C.P., et al., Presynaptic serotonergic markers in community-acquired cases of Alzheimer's disease: correlations with depression and neuroleptic medication. J Neurochem, 1996. 66(4): p. 1592-8.

487. D'Amato, R.J., et al., Aminergic systems in Alzheimer's disease and Parkinson's disease. Ann Neurol, 1987. 22(2): p. 229-36.

488. Ouchi, Y., et al., Altered Brain Serotonin Transporter and Associated Glucose Metabolism in Alzheimer Disease. J Nucl Med, 2009.

489. Tejani-Butt, S.M., J. Yang, and A.C. Pawlyk, Altered serotonin transporter sites in Alzheimer's disease raphe and hippocampus. Neuroreport, 1995. 6(8): p. 1207-10.

490. Thomas, A.J., et al., A study of the serotonin transporter in the prefrontal cortex in late-life depression and Alzheimer's disease with and without depression. Neuropathol Appl Neurobiol, 2006. 32(3): p. 296-303.

491. Kepe, V., et al., Serotonin $1 A$ receptors in the living brain of Alzheimer's disease patients. Proc Natl Acad Sci U S A, 2006. 103(3): p. 702-7.

492. Nitsch, R.M., et al., Serotonin 5-HT2a and 5-HT2c receptors stimulate amyloid precursor protein ectodomain secretion. J Biol Chem, 1996. 271(8): p. 4188-94.

493. Lezoualc'h, F. and S.J. Robert, The serotonin 5-HT4 receptor and the amyloid precursor protein processing. Exp Gerontol, 2003. 38(1-2): p. 159-66.

494. Eglen, R.M., et al., Central 5-HT4 receptors. Trends Pharmacol Sci, 1995. 16(11): p. 391-8.

495. Nelson, R.L., et al., Prophylactic treatment with paroxetine ameliorates behavioral deficits and retards the development of amyloid and tau pathologies in 3xTgAD mice. Exp Neurol, 2007. 205(1): p. 166-76.

496. Pakaski, M., et al., Imipramine and citalopram facilitate amyloid precursor protein secretion in vitro. Neurochem Int, 2005. 47(3): p. 190-5.

497. Katzman, R., Education and the prevalence of dementia and Alzheimer's disease. Neurology, 1993. 43(1): p. 13-20.

498. Stern, Y., What is cognitive reserve? Theory and research application of the reserve concept. J Int Neuropsychol Soc, 2002. 8(3): p. 448-60.

499. Olin, J.T., et al., Provisional diagnostic criteria for depression of Alzheimer disease: rationale and background. Am J Geriatr Psychiatry, 2002. 10(2): p. 129-41.

500. Phillips, H.S., et al., BDNF mRNA is decreased in the hippocampus of individuals with Alzheimer's disease. Neuron, 1991. 7(5): p. 695-702.

501. Wang, R., et al., Presenilin 1 familial Alzheimer's disease mutation leads to defective associative learning and impaired adult neurogenesis. Neuroscience, 2004. 126(2): p. 305-12.

502. Nagahara, A.H., et al., Neuroprotective effects of brain-derived neurotrophic factor in rodent and primate models of Alzheimer's disease. Nat Med, 2009. 15(3): p. 3317.

503. Tanzi, R.E. and L. Bertram, Twenty years of the Alzheimer's disease amyloid hypothesis: a genetic perspective. Cell, 2005. 120(4): p. 545-55.

504. Chouliaras, L., et al., Gene-environment interaction research and transgenic mouse models of Alzheimer's disease. Int J Alzheimers Dis, 2010. 2010.

505. Cuadrado-Tejedor, M., et al., Chronic Mild Stress Accelerates the Onset and Progression of the Alzheimer's Disease Phenotype in Tg2576 Mice. J Alzheimers Dis, 2011.

506. Jeong, Y.H., et al., Environmental enrichment compensates for the effects of stress on disease progression in Tg2576 mice, an Alzheimer's disease model. J Neurochem, 2011. 119(6): p. 1282-93. 
507. Huang, H.J., et al., Long-term social isolation exacerbates the impairment of spatial working memory in APP/PS1 transgenic mice. Brain Res, 2011. 1371: p. 150-60.

508. Devi, L., et al., Sex- and brain region-specific acceleration of beta-amyloidogenesis following behavioral stress in a mouse model of Alzheimer's disease. Mol Brain, 2010. 3: p. 34.

509. Fumagalli, F., et al., Stress during development: Impact on neuroplasticity and relevance to psychopathology. Prog Neurobiol, 2007. 81(4): p. 197-217.

510. Behan, A.T., et al., Evidence of female-specific glial deficits in the hippocampus in a mouse model of prenatal stress. Eur Neuropsychopharmacol, 2011. 21(1): p. 719.

511. Mueller, B.R. and T.L. Bale, Early prenatal stress impact on coping strategies and learning performance is sex dependent. Physiol Behav, 2007. 91(1): p. 55-65.

512. Bustamante, C., et al., Effects of prenatal stress and exercise on dentate granule cells maturation and spatial memory in adolescent mice. Int J Dev Neurosci, 2011. 28(7): p. 605-9.

513. Son, G.H., et al., Maternal stress produces learning deficits associated with impairment of NMDA receptor-mediated synaptic plasticity. J Neurosci, 2006. 26(12): p. 3309-18.

514. Mueller, B.R. and T.L. Bale, Sex-specific programming of offspring emotionality after stress early in pregnancy. J Neurosci, 2008. 28(36): p. 9055-65.

515. Mastroeni, D., et al., Epigenetic mechanisms in Alzheimer's disease. Neurobiol Aging, 2011. 32(7): p. 1161-80.

516. Kaminsky, Z., S.C. Wang, and A. Petronis, Complex disease, gender and epigenetics. Ann Med, 2006. 38(8): p. 530-44.

517. Goldberg, A.D., C.D. Allis, and E. Bernstein, Epigenetics: a landscape takes shape. Cell, 2007. 128(4): p. 635-8.

518. Lahiri, D.K., et al., Early-life events may trigger biochemical pathways for Alzheimer's disease: the "LEARn" model. Biogerontology, 2008. 9(6): p. 375-9.

519. Landrigan, P.J., et al., Early environmental origins of neurodegenerative disease in later life. Environ Health Perspect, 2005. 113(9): p. 1230-3.

520. Waterland, R.A. and K.B. Michels, Epigenetic epidemiology of the developmental origins hypothesis. Annu Rev Nutr, 2007. 27: p. 363-88.

521. Dolinoy, D.C., et al., Metastable epialleles, imprinting, and the fetal origins of adult diseases. Pediatr Res, 2007. 61(5 Pt 2): p. 30R-37R.

522. Vanmierlo, T., et al., Liver $X$ receptor activation restores memory in aged $A D$ mice without reducing amyloid. Neurobiol Aging, 2011. 32(7): p. 1262-72.

523. Bruno, O., et al., GEBR-7b, a novel PDE4D selective inhibitor that improves memory in rodents at non-emetic doses. Br J Pharmacol, 2011. 164(8): p. 20542063.

524. Hooper, N., C. Fraser, and T.W. Stone, Effects of purine analogues on spontaneous alternation in mice. Psychopharmacology (Berl), 1996. 123(3): p. 2507.

525. Conrad, C.D., et al., Chronic stress impairs rat spatial memory on the Y maze, and this effect is blocked by tianeptine pretreatment. Behav Neurosci, 1996. 110(6): p. 1321-34.

526. Melnikova, T., et al., Cycloxygenase-2 activity promotes cognitive deficits but not increased amyloid burden in a model of Alzheimer's disease in a sex-dimorphic pattern. Neuroscience, 2006. 141(3): p. 1149-62.

527. Shepherd, J.K., et al., Behavioural and pharmacological characterisation of the elevated "zero-maze" as an animal model of anxiety. Psychopharmacology (Berl), 1994. 116(1): p. 56-64. 
528. van Donkelaar, E.L., et al., Acute tryptophan depletion in C57BL/6 mice does not induce central serotonin reduction or affective behavioural changes. Neurochem Int, 2010. 56(1): p. 21-34.

529. Porsolt, R.D., A. Bertin, and M. Jalfre, "Behavioural despair" in rats and mice: strain differences and the effects of imipramine. Eur J Pharmacol, 1978. 51(3): p. 291-4.

530. Ferretti, M.T., et al., Intracellular Abeta-oligomers and early inflammation in a model of Alzheimer's disease. Neurobiol Aging, 2011.

531. Grant, S.M., et al., Abeta immunoreactive material is present in several intracellular compartments in transfected, neuronally differentiated, P19 cells expressing the human amyloid beta-protein precursor. J Alzheimers Dis, 2000. 2(3-4): p. 207-22.

532. Franklin, K.B.J. and G. Paxinos, The mouse brain in stereotaxic coordinates. 3rd ed. 2008, Amsterdam ; Boston: Elsevier Academic Press. 1 v. (unpaged).

533. Mahar, I., et al., Subchronic peripheral neuregulin-1 increases ventral hippocampal neurogenesis and induces antidepressant-like effects. PLoS One, 2011. 6(10): p. e26610.

534. Mulder, M., et al., LDL receptor deficiency results in decreased cell proliferation and presynaptic bouton density in the murine hippocampus. Neurosci Res, 2007. 59(3): p. 251-6.

535. Strackx, E., et al., A combined behavioral and morphological study on the effects of fetal asphyxia on the nigrostriatal dopaminergic system in adult rats. Exp Neurol, 2008. 211(2): p. 413-22.

536. Chouliaras, L., et al., Caloric restriction attenuates age-related changes of DNA methyltransferase 3a in mouse hippocampus. Brain Behav Immun, 2011. 25(4): p. 616-23.

537. Gundersen, H.J., et al., The new stereological tools: disector, fractionator, nucleator and point sampled intercepts and their use in pathological research and diagnosis. APMIS, 1988. 96(10): p. 857-81.

538. Schmitz, C. and P.R. Hof, Recommendations for straightforward and rigorous methods of counting neurons based on a computer simulation approach. J Chem Neuroanat, 2000. 20(1): p. 93-114.

539. Kreczmanski, P., et al., Volume, neuron density and total neuron number in five subcortical regions in schizophrenia. Brain, 2007. 130(Pt 3): p. 678-92.

540. Kravitz, D.J., et al., A new neural framework for visuospatial processing. Nat Rev Neurosci, 2011. 12(4): p. 217-30.

541. Lalonde, R., H.D. Kim, and K. Fukuchi, Exploratory activity, anxiety, and motor coordination in bigenic APPswe + PS1/DeltaE9 mice. Neurosci Lett, 2004. 369(2): p. $156-61$.

542. Van den Hove, D., et al., Differential effects of prenatal stress in 5-Htt deficient mice: towards molecular mechanisms of gene $x$ environment interactions. PLoS One, 2011. 6(8): p. e22715.

543. Leblhuber, F., et al., Age and sex differences of dehydroepiandrosterone sulfate (DHEAS) and cortisol (CRT) plasma levels in normal controls and Alzheimer's disease (AD). Psychopharmacology (Berl), 1993. 111(1): p. 23-6.

544. Clinton, L.K., et al., Age-dependent sexual dimorphism in cognition and stress response in the $3 x T g-A D$ mice. Neurobiol Dis, 2007. 28(1): p. 76-82.

545. Gimenez-Llort, L., et al., Gender-Specific Neuroimmunoendocrine Response to Treadmill Exercise in 3xTg-AD Mice. Int J Alzheimers Dis, 2010. 2010: p. 128354.

546. Richardson, H.N., et al., Exposure to repetitive versus varied stress during prenatal development generates two distinct anxiogenic and neuroendocrine profiles in adulthood. Endocrinology, 2006. 147(5): p. 2506-17.

547. Van den Hove, D.L., et al., Prenatal restraint stress and long-term affective consequences. Dev Neurosci, 2005. 27(5): p. 313-20. 
548. van den Hove, D.L., et al., Maternal stress-induced reduction in birth weight as a marker for adult affective state. Front Biosci (Elite Ed), 2010. 2: p. 43-6.

549. Darnaudery, M. and S. Maccari, Epigenetic programming of the stress response in male and female rats by prenatal restraint stress. Brain Res Rev, 2008. 57(2): p. 571-85.

550. Bowman, R.E., et al., Sexually dimorphic effects of prenatal stress on cognition, hormonal responses, and central neurotransmitters. Endocrinology, 2004. 145(8): p. 3778-87.

551. Maccari, S. and S. Morley-Fletcher, Effects of prenatal restraint stress on the hypothalamus-pituitary-adrenal axis and related behavioural and neurobiological alterations. Psychoneuroendocrinology, 2007. 32 Suppl 1: p. S10-5.

552. McCormick, C.M., et al., Sex-specific effects of prenatal stress on hypothalamicpituitary-adrenal responses to stress and brain glucocorticoid receptor density in adult rats. Brain Res Dev Brain Res, 1995. 84(1): p. 55-61.

553. Zuena, A.R., et al., Prenatal restraint stress generates two distinct behavioral and neurochemical profiles in male and female rats. PLoS One, 2008. 3(5): p. e2170.

554. Van den Hove, D.L., et al., Prenatal stress and neonatal rat brain development. Neuroscience, 2006. 137(1): p. 145-55.

555. Harris, A. and J. Seckl, Glucocorticoids, prenatal stress and the programming of disease. Horm Behav, 2010. 59(3): p. 279-89.

556. Pistell, P.J., M. Zhu, and D.K. Ingram, Acquisition of conditioned taste aversion is impaired in the amyloid precursor protein/presenilin 1 mouse model of Alzheimer's disease. Neuroscience, 2008. 152(3): p. 594-600.

557. Weinstock, M., Sex-dependent changes induced by prenatal stress in cortical and hippocampal morphology and behaviour in rats: an update. Stress, 2011. 14(6): p. 604-13.

558. Butkevich, I.P. and E.A. Vershinina, Maternal stress differently alters nociceptive behaviors in the formalin test in adult female and male rats. Brain Res, 2003. 961(1): p. 159-65.

559. Muhammad, A. and B. Kolb, Mild prenatal stress-modulated behavior and neuronal spine density without affecting amphetamine sensitization. Dev Neurosci, 2011. 33(2): p. 85-98.

560. Schmitz, C., et al., Depression: reduced number of granule cells in the hippocampus of female, but not male, rats due to prenatal restraint stress. Mol Psychiatry, 2002. 7(7): p. 810-3.

561. Ferretti, M.T., et al., Transgenic mice as a model of pre-clinical Alzheimer's disease. Curr Alzheimer Res, 2011. 8(1): p. 4-23.

562. Leon, W.C., et al., A novel transgenic rat model with a full Alzheimer's-like amyloid pathology displays pre-plaque intracellular amyloid-beta-associated cognitive impairment. J Alzheimers Dis, 2010. 20(1): p. 113-26.

563. Ke, X., et al., Uteroplacental insufficiency affects epigenetic determinants of chromatin structure in brains of neonatal and juvenile IUGR rats. Physiol Genomics, 2006. 25(1): p. 16-28.

564. Feng, J., et al., Dynamic expression of de novo DNA methyltransferases Dnmt3a and Dnmt3b in the central nervous system. J Neurosci Res, 2005. 79(6): p. 734-46.

565. Kriaucionis, S. and N. Heintz, The nuclear DNA base 5-hydroxymethylcytosine is present in Purkinje neurons and the brain. Science, 2009. 324(5929): p. 929-30.

566. Brown, S.E., et al., Regional-specific global cytosine methylation and DNA methyltransferase expression in the adult rat hippocampus. Neurosci Lett, 2008. 440(1): p. 49-53.

567. Chouliaras, L., et al., Prevention of age-related changes in hippocampal levels of 5methylcytidine by caloric restriction. Neurobiol Aging, 2012. 33(8): p. 1672-81. 
568. Tahiliani, M., et al., Conversion of 5-methylcytosine to 5-hydroxymethylcytosine in mammalian DNA by MLL partner TET1. Science, 2009. 324(5929): p. 930-5.

569. Morgan, A.R., et al., Association analysis of 528 intra-genic SNPs in a region of chromosome 10 linked to late onset Alzheimer's disease. Am J Med Genet B Neuropsychiatr Genet, 2008. 147B(6): p. 727-31.

570. Jin, S.G., S. Kadam, and G.P. Pfeifer, Examination of the specificity of DNA methylation profiling techniques towards 5-methylcytosine and 5hydroxymethylcytosine. Nucleic Acids Res, 2010. 38(11): p. e125.

571. van den Hove, D.L., L. Chouliaras, and B.P. Rutten, The role of 5hydroxymethylcytosine in aging and Alzheimer's disease: current status and prospects for future studies. Curr Alzheimer Res, 2012. 9(5): p. 545-9.

572. Mastroeni, D., et al., Epigenetic changes in Alzheimer's disease: decrements in DNA methylation. Neurobiol Aging, 2010. 31(12): p. 2025-37.

573. Mastroeni, D., et al., Epigenetic differences in cortical neurons from a pair of monozygotic twins discordant for Alzheimer's disease. PLoS One, 2009. 4(8): p. e6617.

574. West, R.L., J.M. Lee, and L.E. Maroun, Hypomethylation of the amyloid precursor protein gene in the brain of an Alzheimer's disease patient. J Mol Neurosci, 1995. 6(2): p. 141-6.

575. Baek, S.H., et al., Exchange of $\mathrm{N}-\mathrm{CoR}$ corepressor and Tip60 coactivator complexes links gene expression by NF-kappaB and beta-amyloid precursor protein. Cell, 2002. 110(1): p. 55-67.

576. Cao, X. and T.C. Sudhof, A transcriptionally [correction of transcriptively] active complex of APP with Fe65 and histone acetyltransferase Tip60. Science, 2001. 293(5527): p. 115-20.

577. von Rotz, R.C., et al., The APP intracellular domain forms nuclear multiprotein complexes and regulates the transcription of its own precursor. J Cell Sci, 2004. 117(Pt 19): p. 4435-48.

578. Letenneur, L., et al., Education and the risk for Alzheimer's disease: sex makes a difference. EURODEM pooled analyses. EURODEM Incidence Research Group. Am J Epidemiol, 2000. 151(11): p. 1064-71.

579. Hirata-Fukae, C., et al., Females exhibit more extensive amyloid, but not tau, pathology in an Alzheimer transgenic model. Brain Res, 2008. 1216: p. 92-103.

580. Wang, J., et al., Gender differences in the amount and deposition of amyloidbeta in APPswe and PS1 double transgenic mice. Neurobiol Dis, 2003. 14(3): p. 318-27.

581. Cunha, C., R. Brambilla, and K.L. Thomas, A simple role for BDNF in learning and memory? Front Mol Neurosci, 2010. 3: p. 1.

582. Coulson, E.J., Does the p75 neurotrophin receptor mediate Abeta-induced toxicity in Alzheimer's disease? J Neurochem, 2006. 98(3): p. 654-60.

583. Fukumoto, N., et al., Sexually dimorphic effect of the Val66Met polymorphism of $B D N F$ on susceptibility to Alzheimer's disease: New data and meta-analysis. Am J Med Genet B Neuropsychiatr Genet, 2010. 153B(1): p. 235-42.

584. Ruiz-Leon, Y. and A. Pascual, Regulation of beta-amyloid precursor protein expression by brain-derived neurotrophic factor involves activation of both the Ras and phosphatidylinositide 3-kinase signalling pathways. Journal of Neurochemistry, 2004. 88(4): p. 1010-1018.

585. Devi, L. and M. Ohno, 7,8-Dihydroxyflavone, a Small-Molecule TrkB Agonist, Reverses Memory Deficits and BACE1 Elevation in a Mouse Model of Alzheimer's Disease. Neuropsychopharmacology, 2011. 37(2): p. 434-44.

586. Tapia-Arancibia, L., et al., New insights into brain BDNF function in normal aging and Alzheimer disease. Brain Res Rev, 2008. 59(1): p. 201-20.

587. Franklin, T.B. and T.S. Perrot-Sinal, Sex and ovarian steroids modulate brainderived neurotrophic factor (BDNF) protein levels in rat hippocampus under 
stressful and non-stressful conditions. Psychoneuroendocrinology, 2006. 31(1): p. 38-48.

588. Lin, Y., et al., Sex differences in the effects of acute and chronic stress and recovery after long-term stress on stress-related brain regions of rats. Cereb Cortex, 2009. 19(9): p. 1978-89.

589. Lopez-Gallardo, M., et al., Maternal deprivation and adolescent cannabinoid exposure impact hippocampal astrocytes, CB1 receptors and brain-derived neurotrophic factor in a sexually dimorphic fashion. Neuroscience, 2012. 204: p. 90-103.

590. Sierksma, A.S., et al., Behavioral and neurobiological effects of prenatal stress exposure in male and female APPswe/PS1 $\triangle E 9$ mice. Neurobiol Aging, 2012.

591. Lessmann, V., K. Gottmann, and M. Malcangio, Neurotrophin secretion: current facts and future prospects. Prog Neurobiol, 2003. 69(5): p. 341-74.

592. Pang, P.T., et al., Cleavage of proBDNF by tPA/plasmin is essential for long-term hippocampal plasticity. Science, 2004. 306(5695): p. 487-91.

593. Teng, H.K., et al., ProBDNF induces neuronal apoptosis via activation of a receptor complex of p75NTR and sortilin. J Neurosci, 2005. 25(22): p. 5455-63.

594. Sartori, C.R., et al., The antidepressive effect of the physical exercise correlates with increased levels of mature BDNF, and proBDNF proteolytic cleavage-related genes, $p 11$ and tPA. Neuroscience, 2011. 180: p. 9-18.

595. Scharfman, H.E. and N.J. MacLusky, Estrogen and brain-derived neurotrophic factor (BDNF) in hippocampus: complexity of steroid hormone-growth factor interactions in the adult CNS. Front Neuroendocrinol, 2006. 27(4): p. 415-35.

596. Monteggia, L.M., et al., Essential role of brain-derived neurotrophic factor in adult hippocampal function. Proc Natl Acad Sci U S A, 2004. 101(29): p. 10827-32.

597. Chakravarthy, B., et al., Amyloid-beta peptides stimulate the expression of the p75(NTR) neurotrophin receptor in SHSY5Y human neuroblastoma cells and $A D$ transgenic mice. J Alzheimers Dis, 2010. 19(3): p. 915-25.

598. Kemppainen, S., et al., Impaired TrkB receptor signaling contributes to memory impairment in APP/PS1 mice. Neurobiol Aging, 2012.

599. Machova, E., et al., Impairment of muscarinic transmission in transgenic APPswe/PS1 $1 E 9$ mice. Neurobiol Aging, 2008. 29(3): p. 368-78.

600. Rodefer, J.S., S.K. Saland, and S.J. Eckrich, Selective phosphodiesterase inhibitors improve performance on the ED/ID cognitive task in rats. Neuropharmacology, 2012. 62(3): p. 1182-90.

601. Barad, M., et al., Rolipram, a type IV-specific phosphodiesterase inhibitor, facilitates the establishment of long-lasting long-term potentiation and improves memory. Proc Natl Acad Sci U S A, 1998. 95(25): p. 15020-5.

602. Navakkode, S., S. Sajikumar, and J.U. Frey, The type IV-specific phosphodiesterase inhibitor rolipram and its effect on hippocampal long-term potentiation and synaptic tagging. J Neurosci, 2004. 24(35): p. 7740-4.

603. Navakkode, S., S. Sajikumar, and J.U. Frey, Mitogen-activated protein kinasemediated reinforcement of hippocampal early long-term depression by the type IVspecific phosphodiesterase inhibitor rolipram and its effect on synaptic tagging. $\mathrm{J}$ Neurosci, 2005. 25(46): p. 10664-70.

604. Walsh, D.M., et al., Naturally secreted oligomers of amyloid beta protein potently inhibit hippocampal long-term potentiation in vivo. Nature, 2002. 416(6880): p. 5359.

605. Yamamoto, M., et al., Hippocampal level of neural specific adenylyl cyclase type I is decreased in Alzheimer's disease. Biochim Biophys Acta, 2000. 1535(1): p. 60-8.

606. Yamamoto-Sasaki, M., et al., Impaired phosphorylation of cyclic AMP response element binding protein in the hippocampus of dementia of the Alzheimer type. Brain Res, 1999. 824(2): p. 300-3. 
607. Bonkale, W.L., et al., Reduced nitric oxide responsive soluble guanylyl cyclase activity in the superior temporal cortex of patients with Alzheimer's disease. Neurosci Lett, 1995. 187(1): p. 5-8.

608. Cowburn, R.F., et al., Adenylyl cyclase activity in postmortem human brain: evidence of altered $G$ protein mediation in Alzheimer's disease. J Neurochem, 1992. 58(4): p. 1409-19.

609. Sabayan, B., N. Zamiri, and S. Farshchizarabi, Phosphodiesterase-5 inhibitors: novel weapons against Alzheimer's disease? Int J Neurosci, 2010. 120(12): p. 74651.

610. Cheng, Y.F., et al., Inhibition of phosphodiesterase-4 reverses memory deficits produced by Abeta25-35 or Abeta1-40 peptide in rats. Psychopharmacology (Berl), 2010. 212(2): p. 181-91.

611. Wang, C., et al., The phosphodiesterase-4 inhibitor rolipram reverses Abetainduced cognitive impairment and neuroinflammatory and apoptotic responses in rats. Int J Neuropsychopharmacol, 2011: p. 1-18.

612. Robichaud, A., et al., Emesis induced by inhibitors of type IV cyclic nucleotide phosphodiesterase (PDE IV) in the ferret. Neuropharmacology, 1999. 38(2): p. 28997.

613. Domek-Lopacinska, K. and J.B. Strosznajder, The effect of selective inhibition of cyclic GMP hydrolyzing phosphodiesterases 2 and 5 on learning and memory processes and nitric oxide synthase activity in brain during aging. Brain Res, 2008. 1216: p. 68-77.

614. Yamada, K., et al., Reduction in the number of NADPH-diaphorase-positive cells in the cerebral cortex and striatum in aged rats. Neurosci Res, 1996. 24(4): p. 393402.

615. Boess, F.G., et al., Inhibition of phosphodiesterase 2 increases neuronal cGMP, synaptic plasticity and memory performance. Neuropharmacology, 2004. 47(7): p. 1081-92.

616. Lakics, V., E.H. Karran, and F.G. Boess, Quantitative comparison of phosphodiesterase mRNA distribution in human brain and peripheral tissues. Neuropharmacology, 2010. 59(6): p. 367-74.

617. Velardez, M.O., et al., Role of phosphodiesterase and protein kinase $G$ on nitric oxide-induced inhibition of prolactin release from the rat anterior pituitary. Eur $\mathrm{J}$ Endocrinol, 2000. 143(2): p. 279-84.

618. White, H., et al., Weight change in Alzheimer's disease. J Am Geriatr Soc, 1996. 44(3): p. 265-72.

619. Gustafson, D., et al., An 18-year follow-up of overweight and risk of Alzheimer disease. Arch Intern Med, 2003. 163(13): p. 1524-8.

620. Ambree, O., et al., Activity changes and marked stereotypic behavior precede Abeta pathology in TgCRND8 Alzheimer mice. Neurobiol Aging, 2006. 27(7): p. 955-64.

621. Touma, C., et al., Age- and sex-dependent development of adrenocortical hyperactivity in a transgenic mouse model of Alzheimer's disease. Neurobiol Aging, 2004. 25(7): p. 893-904.

622. Ennaceur, A. and J. Delacour, A new one-trial test for neurobiological studies of memory in rats. 1: Behavioral data. Behav Brain Res, 1988. 31(1): p. 47-59.

623. Verfaellie, M., et al., Not all repetition is alike: different benefits of repetition in amnesia and normal memory. J Int Neuropsychol Soc, 2008. 14(3): p. 365-72.

624. Liebenberg, N., et al., Antidepressant-like properties of phosphodiesterase type 5 inhibitors and cholinergic dependency in a genetic rat model of depression. Behav Pharmacol, 2010. 21(5-6): p. 540-7. 
625. Liebenberg, $\mathrm{N}$., et al., Chronic treatment with the phosphodiesterase type 5 inhibitors sildenafil and tadalafil display anxiolytic effects in Flinders Sensitive Line rats. Metab Brain Dis, 2012.

626. Li, S., E. Chung, and R.M. Quock, Role of cyclic GMP in nitrous-oxide-induced anxiolytic-like behavior in the mouse light-dark exploration test. Behav Neurosci, 2004. 118(3): p. 648-52.

627. Berton, O. and E.J. Nestler, New approaches to antidepressant drug discovery: beyond monoamines. Nat Rev Neurosci, 2006. 7(2): p. 137-51.

628. Li, Y.F., et al., Antidepressant- and anxiolytic-like effects of the phosphodiesterase4 inhibitor rolipram on behavior depend on cyclic AMP response element binding protein-mediated neurogenesis in the hippocampus. Neuropsychopharmacology, 2009. 34(11): p. 2404-19.

629. Kurt, M., et al., Effect of sildenafil on anxiety in the plus-maze test in mice. Pol J Pharmacol, 2004. 56(3): p. 353-7.

630. Masood, A., et al., Anxiolytic effects of phosphodiesterase-2 inhibitors associated with increased cGMP signaling. J Pharmacol Exp Ther, 2009. 331(2): p. 690-9.

631. Pedersen, W.A. and E.R. Flynn, Insulin resistance contributes to aberrant stress responses in the Tg2576 mouse model of Alzheimer's disease. Neurobiol Dis, 2004. 17(3): p. 500-6.

632. Cuadrado-Tejedor, M., et al., Sildenafil restores cognitive function without affecting beta-amyloid burden in a mouse model of Alzheimer's disease. $\mathrm{Br} \mathrm{J}$ Pharmacol, 2011. 164(8): p. 2029-41.

633. Kwak, Y.D., et al., Differential regulation of BACE1 expression by oxidative and nitrosative signals. Mol Neurodegener, 2011. 6: p. 17.

634. Austin, S.A., A.V. Santhanam, and Z.S. Katusic, Endothelial nitric oxide modulates expression and processing of amyloid precursor protein. Circ Res, 2010. 107(12): p. $1498-502$.

635. Nibuya, M., E.J. Nestler, and R.S. Duman, Chronic antidepressant administration increases the expression of CAMP response element binding protein (CREB) in rat hippocampus. J Neurosci, 1996. 16(7): p. 2365-72.

636. Puerta, E., et al., Sildenafil protects against 3-nitropropionic acid neurotoxicity through the modulation of calpain, CREB, and BDNF. Neurobiol Dis, 2010. 38(2): p. 237-45.

637. Tong, L.Q., et al., beta-Amyloid-(1-42) impairs activity-dependent cAMP-response element-binding protein signaling in neurons at concentrations in which cell survival is not compromised. Journal of Biological Chemistry, 2001. 276(20): p. 1730117306.

638. Reese, L.C., et al., Selective induction of calcineurin activity and signaling by oligomeric amyloid beta. Aging Cell, 2008. 7(6): p. 824-35.

639. Li, N. and G.T. Liu, The novel squamosamide derivative FLZ enhances $B D N F / T r k B / C R E B$ signaling and inhibits neuronal apoptosis in APP/PS1 mice. Acta Pharmacol Sin, 2010. 31(3): p. 265-72.

640. Arvanitis, D.N., et al., High intracellular concentrations of amyloid-beta block nuclear translocation of phosphorylated CREB. J Neurochem, 2007. 103(1): p. 21628.

641. Hou, Y., et al., Anti-depressant natural flavonols modulate BDNF and beta amyloid in neurons and hippocampus of double TgAD mice. Neuropharmacology, 2010. 58(6): p. 911-20.

642. Chen, Q., et al., A novel neurotrophic drug for cognitive enhancement and Alzheimer's disease. PLoS One, 2011. 6(12): p. e27865.

643. Liu, H.L., et al., Treadmill exercise prevents decline in spatial learning and memory in APP/PS1 transgenic mice through improvement of hippocampal long-term potentiation. Behav Brain Res, 2011. 218(2): p. 308-14. 
644. Szapacs, M.E., A.L. Numis, and A.M. Andrews, Late onset loss of hippocampal 5$H T$ and NE is accompanied by increases in BDNF protein expression in mice coexpressing mutant APP and PS1. Neurobiol Dis, 2004. 16(3): p. 572-80.

645. Ma, L., et al., Cyclic AMP induces functional presynaptic boutons in hippocampal CA3-CA1 neuronal cultures. Nat Neurosci, 1999. 2(1): p. 24-30.

646. Smith, D.L., et al., Reversal of long-term dendritic spine alterations in Alzheimer disease models. Proc Natl Acad Sci U S A, 2009. 106(39): p. 16877-82.

647. West, M.J., et al., Synaptic contact number and size in stratum radiatum CA1 of APP/PS1DeltaE9 transgenic mice. Neurobiol Aging, 2009. 30(11): p. 1756-76.

648. Mukaetova-Ladinska, E.B., et al., Staging of cytoskeletal and beta-amyloid changes in human isocortex reveals biphasic synaptic protein response during progression of Alzheimer's disease. Am J Pathol, 2000. 157(2): p. 623-36.

649. Boncristiano, S., et al., Neocortical synaptic bouton number is maintained despite robust amyloid deposition in APP23 transgenic mice. Neurobiol Aging, 2005. 26(5): p. 607-13.

650. Scheff, S.W. and D.A. Price, Synaptic pathology in Alzheimer's disease: a review of ultrastructural studies. Neurobiol Aging, 2003. 24(8): p. 1029-46.

651. Mucke, L., et al., Synaptotrophic effects of human amyloid beta protein precursors in the cortex of transgenic mice. Brain Res, 1994. 666(2): p. 151-67.

652. Francis, S.H., M.A. Blount, and J.D. Corbin, Mammalian cyclic nucleotide phosphodiesterases: molecular mechanisms and physiological functions. Physiol Rev, 2011. 91(2): p. 651-90.

653. Garzon, D.J. and M. Fahnestock, Oligomeric amyloid decreases basal levels of brain-derived neurotrophic factor (BDNF) mRNA via specific downregulation of $B D N F$ transcripts IV and $V$ in differentiated human neuroblastoma cells. J Neurosci, 2007. 27(10): p. 2628-35.

654. Rutten, K., et al., Time-dependent involvement of cAMP and cGMP in consolidation of object memory: studies using selective phosphodiesterase type 2, 4 and 5 inhibitors. Eur J Pharmacol, 2007. 558(1-3): p. 107-12.

655. Rutten, K., et al., Phosphodiesterase inhibitors enhance object memory independent of cerebral blood flow and glucose utilization in rats. Neuropsychopharmacology, 2009. 34(8): p. 1914-25.

656. van Donkelaar, E.L., et al., Phosphodiesterase 2 and 5 inhibition attenuates the object memory deficit induced by acute tryptophan depletion. Eur J Pharmacol, 2008. 600(1-3): p. 98-104.

657. Reneerkens, O.A., et al., Phosphodiesterase type 5 (PDE5) inhibition improves object recognition memory: Indications for central and peripheral mechanisms. Neurobiol Learn Mem, 2012.

658. Selkoe, D.J., Alzheimer's disease is a synaptic failure. Science, 2002. 298(5594): p. 789-91.

659. Blokland, A., R. Schreiber, and J. Prickaerts, Improving memory: a role for phosphodiesterases. Curr Pharm Des, 2006. 12(20): p. 2511-23.

660. Wilcox, K.C., et al., Abeta oligomer-induced synapse degeneration in Alzheimer's disease. Cell Mol Neurobiol, 2011. 31(6): p. 939-48.

661. Hanger, D.P., B.H. Anderton, and W. Noble, Tau phosphorylation: the therapeutic challenge for neurodegenerative disease. Trends Mol Med, 2009. 15(3): p. 112-9.

662. Grimes, C.A. and R.S. Jope, The multifaceted roles of glycogen synthase kinase 3beta in cellular signaling. Prog Neurobiol, 2001. 65(4): p. 391-426.

663. Creighton, J., et al., Spectrin-anchored phosphodiesterase 4D4 restricts CAMP from disrupting microtubules and inducing endothelial cell gap formation. J Cell Sci, 2008. 121(Pt 1): p. 110-9. 
664. Zhu, B., et al., Protein kinase A phosphorylation of tau-serine 214 reorganizes microtubules and disrupts the endothelial cell barrier. Am J Physiol Lung Cell Mol Physiol, 2010. 299(4): p. L493-501.

665. Burgin, A.B., et al., Design of phosphodiesterase 4D (PDE4D) allosteric modulators for enhancing cognition with improved safety. Nat Biotechnol, 2009. 28(1): p. 63-70.

666. Van der Heyden, J.A., T.J. Zethof, and B. Olivier, Stress-induced hyperthermia in singly housed mice. Physiol Behav, 1997. 62(3): p. 463-70.

667. Prickaerts, J., et al., Differential BDNF Responses of Triple Versus Dual Reuptake Inhibition in Neuronal and Astrocytoma Cells as well as in Rat Hippocampus and Prefrontal Cortex. Journal of Molecular Neuroscience, 2012: p. 1-9.

668. Cryan, J.F. and A. Holmes, The ascent of mouse: advances in modelling human depression and anxiety. Nat Rev Drug Discov, 2005. 4(9): p. 775-90.

669. Willner, P., et al., Reduction of sucrose preference by chronic unpredictable mild stress, and its restoration by a tricyclic antidepressant. Psychopharmacology (Berl), 1987. 93(3): p. 358-64.

670. Vanmierlo, T., et al., Cerebral accumulation of dietary derivable plant sterols does not interfere with memory and anxiety related behavior in Abcg5-/- mice. Plant Foods Hum Nutr, 2011. 66(2): p. 149-56.

671. Prickaerts, J., et al., Transgenic mice overexpressing glycogen synthase kinase 3beta: a putative model of hyperactivity and mania. J Neurosci, 2006. 26(35): p. 9022-9.

672. Antonawich, F.J., et al., Nesting and shredding behavior as an indicator of hippocampal ischemic damage. Brain Res, 1997. 764(1-2): p. 249-52.

673. Deacon, R.M., A. Croucher, and J.N. Rawlins, Hippocampal cytotoxic lesion effects on species-typical behaviours in mice. Behav Brain Res, 2002. 132(2): p. 203-13.

674. Deacon, R., Assessing burrowing, nest construction, and hoarding in mice. J Vis Exp, 2012(59): p. e2607.

675. Ballard, T.M., et al., Severe impairment of NMDA receptor function in mice carrying targeted point mutations in the glycine binding site results in drug-resistant nonhabituating hyperactivity. J Neurosci, 2002. 22(15): p. 6713-23.

676. Sierksma, A.S., et al., Effects of prenatal stress exposure on soluble Abeta and brain-derived neurotrophic factor signaling in male and female APPswe/PS1 $\triangle E 9$ mice. Neurochem Int, 2012.

677. Akkerman, S., et al., Object recognition testing: Methodological considerations on exploration and discrimination measures. Behav Brain Res, 2012.

678. Sierksma, A.S., et al., Chronic phosphodiesterase type 2 inhibition improves memory in the APPswe/PS1 $\triangle E 9$ mouse model of Alzheimer's disease. Neuropharmacology, 2012.

679. Li, Y.F., et al., Phosphodiesterase-4D knock-out and RNA interference-mediated knock-down enhance memory and increase hippocampal neurogenesis via increased cAMP signaling. J Neurosci, 2011. 31(1): p. 172-83.

680. Courtney, S.M., et al., An area specialized for spatial working memory in human frontal cortex. Science, 1998. 279(5355): p. 1347-1351.

681. Frye, C.A. and A.A. Walf, Progesterone reduces depression-like behavior in a murine model of Alzheimer's Disease. Age (Dordr), 2009. 31(2): p. 143-53.

682. Good, M. and R.C. Honey, Dissociable effects of selective lesions to hippocampal subsystems on exploratory behavior, contextual learning, and spatial learning. Behav Neurosci, 1997. 111(3): p. 487-93.

683. Aalten, P., et al., Consistency of neuropsychiatric syndromes across dementias: results from the European Alzheimer Disease Consortium. Part II. Dement Geriatr Cogn Disord, 2008. 25(1): p. 1-8. 
684. Kang, H.S., et al., Neuropsychiatric symptoms in korean patients with Alzheimer's disease: exploratory factor analysis and confirmatory factor analysis of the neuropsychiatric inventory. Dement Geriatr Cogn Disord, 2010. 29(1): p. 82-7.

685. Van Dam, D., et al., Age-dependent cognitive decline in the APP23 model precedes amyloid deposition. European Journal of Neuroscience, 2003. 17(2): p. 388-396.

686. Lalonde, R., et al., Transgenic mice expressing the betaAPP695SWE mutation: effects on exploratory activity, anxiety, and motor coordination. Brain Res, 2003. 977(1): p. 38-45.

687. Zhang, H.T., et al., Antidepressant-like profile and reduced sensitivity to rolipram in mice deficient in the PDE4D phosphodiesterase enzyme. Neuropsychopharmacology, 2002. 27(4): p. 587-95.

688. Caccamo, A., et al., CBP gene transfer increases BDNF levels and ameliorates learning and memory deficits in a mouse model of Alzheimer's disease. Proc Natl Acad Sci U S A, 2010. 107(52): p. 22687-92.

689. Vo, N. and R.H. Goodman, CREB-binding protein and p300 in transcriptional regulation. J Biol Chem, 2001. 276(17): p. 13505-8.

690. Saura, C.A., et al., Loss of presenilin function causes impairments of memory and synaptic plasticity followed by age-dependent neurodegeneration. Neuron, 2004. 42(1): p. 23-36.

691. Boyles, R.S., et al., Presenilin controls CBP levels in the adult Drosophila central nervous system. PLoS One, 2010. 5(12): p. e14332.

692. Francis, Y.I., A. Stephanou, and D.S. Latchman, CREB-binding protein activation by presenilin 1 but not by its M146L mutant. Neuroreport, 2006. 17(9): p. 917-21.

693. Walker, M.P., et al., Reversible epigenetic histone modifications and Bdnf expression in neurons with aging and from a mouse model of Alzheimer's disease. Age (Dordr), 2012.

694. Jansen, D., et al., Cholesterol and Synaptic Compensatory Mechanisms in Alzheimer's Disease Mice Brain During Aging. J Alzheimers Dis, 2012.

695. Inestrosa, N., et al., Tetrahydrohyperforin prevents cognitive deficit, $A \hat{l}^{2}$ deposition, tau phosphorylation and synaptotoxicity in the APPswe/PSEN1 l'"E9 model of Alzheimer's disease: a possible effect on APP processing. Translational Psychiatry, 2010. 1(7): p. e20.

696. Lee, H.G., et al., Microglial P2X(7) receptor expression is accompanied by neuronal damage in the cerebral cortex of the APPswe/PS1 $\triangle E 9$ mouse model of Alzheimer's disease. Exp Mol Med, 2011. 43(1): p. 7-14.

697. Hiltunen, M., et al., Contribution of genetic and dietary insulin resistance to Alzheimer phenotype in APP/PS1 transgenic mice. J Cell Mol Med, 2012. 16(6): p. 1206-22.

698. Malm, T.M., et al., Pyrrolidine dithiocarbamate activates Akt and improves spatial learning in APP/PS1 mice without affecting beta-amyloid burden. J Neurosci, 2007. 27(14): p. 3712-21.

699. Schaeffer, E.L., M. Figueiro, and W.F. Gattaz, Insights into Alzheimer disease pathogenesis from studies in transgenic animal models. Clinics (Sao Paulo), 2011. 66 Suppl 1: p. 45-54.

700. Kessing, L.V., J.L. Forman, and P.K. Andersen, Do continued antidepressants protect against dementia in patients with severe depressive disorder? Int Clin Psychopharmacol, 2011. 26(6): p. 316-22.

701. Mossello, E., et al., Is antidepressant treatment associated with reduced cognitive decline in Alzheimer's disease? Dement Geriatr Cogn Disord, 2008. 25(4): p. 3729. 
702. Mowla, A., et al., Does serotonin augmentation have any effect on cognition and activities of daily living in Alzheimer's dementia? A double-blind, placebo-controlled clinical trial. J Clin Psychopharmacol, 2007. 27(5): p. 484-7.

703. Cirrito, J.R., et al., Serotonin signaling is associated with lower amyloid-beta levels and plaques in transgenic mice and humans. Proc Natl Acad Sci U S A, 2011. 108(36): p. 14968-73.

704. Aboukhatwa, M. and Y. Luo, Antidepressants modulate intracellular amyloid peptide species in N2a neuroblastoma cells. J Alzheimers Dis, 2011. 24(2): p. 22134.

705. Keowkase, R., M. Aboukhatwa, and Y. Luo, Fluoxetine protects against amyloidbeta toxicity, in part via daf-16 mediated cell signaling pathway, in Caenorhabditis elegans. Neuropharmacology, 2010. 59(4-5): p. 358-65.

706. Sierksma, A.S., et al., Improvement of spatial memory function in APPswe/PS1 $1 E 9$ mice after chronic inhibition of phosphodiesterase type 4D. In preparation, 2012.

707. Kobayashi, K., Y. Ikeda, and H. Suzuki, Behavioral destabilization induced by the selective serotonin reuptake inhibitor fluoxetine. Mol Brain, 2011. 4: p. 12.

708. Coupland, N.J., C.J. Bell, and J.P. Potokar, Serotonin reuptake inhibitor withdrawal. J Clin Psychopharmacol, 1996. 16(5): p. 356-62.

709. Haddad, P., The SSRI discontinuation syndrome. Journal of Psychopharmacology, 1998. 12(3): p. 305-313.

710. Price, J.S., et al., A comparison of the post-marketing safety of four selective serotonin re-uptake inhibitors including the investigation of symptoms occurring on withdrawal. British Journal of Clinical Pharmacology, 1996. 42(6): p. 757-763.

711. Izumi, J., et al., Evidence for a depressive-like state induced by repeated saline injections in Fischer 344 rats. Pharmacol Biochem Behav, 1997. 57(4): p. 883-8.

712. Minkeviciene, R., et al., Amyloid beta-induced neuronal hyperexcitability triggers progressive epilepsy. J Neurosci, 2009. 29(11): p. 3453-62.

713. Ziyatdinova, S., et al., Spontaneous epileptiform discharges in a mouse model of Alzheimer's disease are suppressed by antiepileptic drugs that block sodium channels. Epilepsy Res, 2011. 94(1-2): p. 75-85.

714. Hsiao, K.K., et al., Age-related CNS disorder and early death in transgenic FVB/N mice overexpressing Alzheimer amyloid precursor proteins. Neuron, 1995. 15(5): $p$. 1203-18.

715. Palop, J.J., et al., Aberrant excitatory neuronal activity and compensatory remodeling of inhibitory hippocampal circuits in mouse models of Alzheimer's disease. Neuron, 2007. 55(5): p. 697-711.

716. Mendez, M.F., et al., Seizures in Alzheimer's disease: clinicopathologic study. J Geriatr Psychiatry Neurol, 1994. 7(4): p. 230-3.

717. Amatniek, J.C., et al., Incidence and predictors of seizures in patients with Alzheimer's disease. Epilepsia, 2006. 47(5): p. 867-72.

718. Hauser, W.A., et al., Seizures and myoclonus in patients with Alzheimer's disease. Neurology, 1986. 36(9): p. 1226-30.

719. Lozsadi, D.A. and A.J. Larner, Prevalence and causes of seizures at the time of diagnosis of probable Alzheimer's disease. Dement Geriatr Cogn Disord, 2006. 22(2): p. 121-4.

720. Rabinowicz, A.L., et al., Transient epileptic amnesia in dementia: a treatable unrecognized cause of episodic amnestic wandering. Alzheimer Dis Assoc Disord, 2000. 14(4): p. 231-3.

721. Sinforiani, E., et al., Memory disturbances and temporal lobe epilepsy simulating Alzheimer's disease: a case report. Funct Neurol, 2003. 18(1): p. 39-41.

722. Hogh, P., et al., Epilepsy presenting as $A D$ : neuroimaging, electroclinical features, and response to treatment. Neurology, 2002. 58(2): p. 298-301. 
723. Palop, J.J. and L. Mucke, Epilepsy and cognitive impairments in Alzheimer disease. Arch Neurol, 2009. 66(4): p. 435-40.

724. Larner, A.J., Presenilin-1 mutation Alzheimer's disease: a genetic epilepsy syndrome? Epilepsy Behav, 2011. 21(1): p. 20-2.

725. Menendez, M., Pathological and clinical heterogeneity of presenilin 1 gene mutations. J Alzheimers Dis, 2004. 6(5): p. 475-82.

726. Cabrejo, L., et al., Phenotype associated with APP duplication in five families. Brain, 2006. 129(Pt 11): p. 2966-76.

727. Montgomery, S.A., Antidepressants and seizures: emphasis on newer agents and clinical implications. Int J Clin Pract, 2005. 59(12): p. 1435-40.

728. Dailey, J.W. and D.K. Naritoku, Antidepressants and seizures: clinical anecdotes overshadow neuroscience. Biochem Pharmacol, 1996. 52(9): p. 1323-9.

729. Preskorn, S.H. and G.A. Fast, Tricyclic antidepressant-induced seizures and plasma drug concentration. J Clin Psychiatry, 1992. 53(5): p. 160-2.

730. Igelstrom, K.M., Preclinical antiepileptic actions of selective serotonin reuptake inhibitors--implications for clinical trial design. Epilepsia, 2012. 53(4): p. 596-605.

731. Kondziella, D. and F. Asztely, Don't be afraid to treat depression in patients with epilepsy! Acta Neurol Scand, 2009. 119(2): p. 75-80.

732. Favale, E., et al., Anticonvulsant effect of fluoxetine in humans. Neurology, 1995. 45(10): p. 1926-7.

733. Specchio, L.M., et al., Citalopram as treatment of depression in patients with epilepsy. Clin Neuropharmacol, 2004. 27(3): p. 133-6.

734. Oke, A., et al., Dose-dependent seizure activity associated with fluoxetine therapy. QJM, 2001. 94(2): p. 113-4.

735. Neely, J.L., Tonic clonic seizures and tachycardia induced by fluoxetine (Prozac) overdose. W V Med J, 1998. 94(5): p. 283-5.

736. Gross, R., et al., Generalized seizures caused by fluoxetine overdose. Am J Emerg Med, 1998. 16(3): p. 328-9.

737. Lange, S.C., R.M. Julien, and G.W. Fowler, Biphasic effects of imipramine in experimental models of epilepsy. Epilepsia, 1976. 17(2): p. 183-95.

738. Perucca, E., et al., Antiepileptic drugs as a cause of worsening seizures. Epilepsia, 1998. 39(1): p. 5-17.

739. Pisani, F., E. Spina, and G. Oteri, Antidepressant drugs and seizure susceptibility: from in vitro data to clinical practice. Epilepsia, 1999. 40 Suppl 10: p. S48-56.

740. Bianchi, M.T. and E.J. Botzolakis, Targeting ligand-gated ion channels in neurology and psychiatry: is pharmacological promiscuity an obstacle or an opportunity? BMC Pharmacol, 2010. 10: p. 3.

741. Velasco, A., et al., Effects of fluoxetine hydrochloride and fluvoxamine maleate on different preparations of isolated guinea pig and rat organ tissues. Gen Pharmacol, 1997. 28(4): p. 509-12.

742. Bolo, N.R., et al., Brain pharmacokinetics and tissue distribution in vivo of fluvoxamine and fluoxetine by fluorine magnetic resonance spectroscopy. Neuropsychopharmacology, 2000. 23(4): p. 428-38.

743. Karson, C.N., et al., Human brain fluoxetine concentrations. J Neuropsychiatry Clin Neurosci, 1993. 5(3): p. 322-9.

744. Browning, R.A., W.E. Hoffmann, and R.L. Simonton, Changes in seizure susceptibility after intracerebral treatment with 5,7-dihydroxytryptamine: role of serotonergic neurons. Ann N Y Acad Sci, 1978. 305: p. 437-56.

745. Jobe, P.C. and H.E. Laird, Neurotransmitter abnormalities as determinants of seizure susceptibility and intensity in the genetic models of epilepsy. Biochem Pharmacol, 1981. 30(23): p. 3137-44. 
746. Holm, P., et al., Plaque deposition dependent decrease in 5-HT2A serotonin receptor in AbetaPPswe/PS1 $\triangle E 9$ amyloid overexpressing mice. J Alzheimers Dis, 2010. 20(4): p. 1201-13.

747. Liu, Y., et al., Amyloid pathology is associated with progressive monoaminergic neurodegeneration in a transgenic mouse model of Alzheimer's disease. J Neurosci, 2008. 28(51): p. 13805-14.

748. Garcia-Marin, V., et al., Diminished perisomatic GABAergic terminals on cortical neurons adjacent to amyloid plaques. Front Neuroanat, 2009. 3: p. 28.

749. Busche, M.A., et al., Clusters of hyperactive neurons near amyloid plaques in a mouse model of Alzheimer's disease. Science, 2008. 321(5896): p. 1686-9.

750. Kuchibhotla, K.V., et al., Abeta plaques lead to aberrant regulation of calcium homeostasis in vivo resulting in structural and functional disruption of neuronal networks. Neuron, 2008. 59(2): p. 214-25.

751. Bendele, R.A., et al., Carcinogenicity studies of fluoxetine hydrochloride in rats and mice. Cancer Res, 1992. 52(24): p. 6931-5.

752. Erenmemisoglu, A., et al., Effect of some antidepressants on glycaemia and insulin levels of normoglycaemic and alloxan-induced hyperglycaemic mice. J Pharm Pharmacol, 1999. 51(6): p. 741-3.

753. Gomes, K.S., et al., Contrasting effects of acute and chronic treatment with imipramine and fluoxetine on inhibitory avoidance and escape responses in mice exposed to the elevated T-maze. Brain Res Bull, 2009. 78(6): p. 323-7.

754. Holladay, J.W., M.J. Dewey, and S.D. Yoo, Pharmacokinetics and antidepressant activity of fluoxetine in transgenic mice with elevated serum alpha-1-acid glycoprotein levels. Drug Metab Dispos, 1998. 26(1): p. 20-4.

755. Hiemke, C. and S. Hartter, Pharmacokinetics of selective serotonin reuptake inhibitors. Pharmacol Ther, 2000. 85(1): p. 11-28.

756. Mowla, A., M. Mosavinasab, and A. Pani, Does fluoxetine have any effect on the cognition of patients with mild cognitive impairment? A double-blind, placebocontrolled, clinical trial. J Clin Psychopharmacol, 2007. 27(1): p. 67-70.

757. Arbus, C., et al., Antidepressant use in Alzheimer's disease patients: results of the REAL.FR cohort. Int Psychogeriatr, 2010. 22(1): p. 120-8.

758. Lyketsos, C.G. and J. Olin, Depression in Alzheimer's disease: overview and treatment. Biol Psychiatry, 2002. 52(3): p. 243-52.

759. Aboukhatwa, M., L. Dosanjh, and Y. Luo, Antidepressants are a rational complementary therapy for the treatment of Alzheimer's disease. Mol Neurodegener, 2010. 5: p. 10.

760. Thompson, S., et al., Efficacy and safety of antidepressants for treatment of depression in Alzheimer's disease: a metaanalysis. Can J Psychiatry, 2007. 52(4): p. 248-55.

761. Banerjee, S., et al., Sertraline or mirtazapine for depression in dementia (HTA$S A D D)$ : a randomised, multicentre, double-blind, placebo-controlled trial. Lancet, 2011. 378(9789): p. 403-11.

762. Weintraub, D., et al., Sertraline for the treatment of depression in Alzheimer disease: week-24 outcomes. Am J Geriatr Psychiatry, 2010. 18(4): p. 332-40.

763. Fraga, M.F., et al., Epigenetic differences arise during the lifetime of monozygotic twins. Proc Natl Acad Sci U S A, 2005. 102(30): p. 10604-9.

764. Hebert, L.E., et al., Is the Risk of Developing Alzheimer's Disease Greater for Women than for Men? American Journal of Epidemiology, 2001. 153(2): p. 132136.

765. Turner, R.S., Alzheimer's disease in man and transgenic mice: females at higher risk. Am J Pathol, 2001. 158(3): p. 797-801.

766. Pike, C.J., et al., Protective actions of sex steroid hormones in Alzheimer's disease. Front Neuroendocrinol, 2009. 30(2): p. 239-58. 
767. Kolodkin, M.H. and A.P. Auger, Sex difference in the expression of DNA methyltransferase $3 a$ in the rat amygdala during development. J Neuroendocrinol, 2011. 23(7): p. 577-83.

768. Westberry, J.M., A.L. Trout, and M.E. Wilson, Epigenetic regulation of estrogen receptor alpha gene expression in the mouse cortex during early postnatal development. Endocrinology, 2011. 151(2): p. 731-40.

769. Zhu, C.W., et al., Utilization of antihypertensives, antidepressants, antipsychotics, and hormones in Alzheimer disease. Alzheimer Dis Assoc Disord, 2011. 25(2): p. 144-8.

770. Xiong, $\mathrm{H}_{\text {., et }}$ al., Biochemical and behavioral characterization of the double transgenic mouse model (APPswe/PS1 $\triangle E 9$ ) of Alzheimer's disease. Neurosci Bull, 2011. 27(4): p. 221-32.

771. Sharp, J., et al., Stress-like responses to common procedures in individually and group-housed female rats. Contemp Top Lab Anim Sci, 2003. 42(1): p. 9-18.

772. Haynes, L.E., D. Barber, and I.J. Mitchell, Chronic antidepressant medication attenuates dexamethasone-induced neuronal death and sublethal neuronal damage in the hippocampus and striatum. Brain Res, 2004. 1026(2): p. 157-67.

773. De Jager, P.L., et al., A genome-wide scan for common variants affecting the rate of age-related cognitive decline. Neurobiol Aging, 2012. 33(5): p. 1017 e1-15.

774. Braidy, N., et al., Recent rodent models for Alzheimer's disease: clinical implications and basic research. J Neural Transm, 2011. 119(2): p. 173-95.

775. Stieler, J.T., et al., The physiological link between metabolic rate depression and tau phosphorylation in mammalian hibernation. PLoS One, 2011. 6(1): p. e14530. 
Summary 
The neurodegenerative disorder Alzheimer's disease (AD) is the most common cause of dementia, yet its etiology remains to be elucidated. Although genetic research has greatly aided the search for the etiopathogenesis of this disorder, genetic factors alone cannot account for the vast majority of $A D$ patients. It has become increasingly clear that other factors, such as the environment, may play a crucial role in driving the onset and/or progression of $A D$. Within this thesis it was explored how exogenous factors, such as stress exposure or treatment with pharmacological agents, can affect the phenotypical expression of AD-related symptoms and neuropathology by using the APPswe/PS1 $1 \mathrm{E} 9$ mouse model of $A D$.

Chapter 1: A general introduction was provided on AD, including a historical overview on its discovery, its symptoms and neuropathology, and the current treatment options. In addition, the mechanism of action of phosphodiesterase inhibitors (PDE-Is) were discussed and their use as potential therapeutic agents for $A D$. Moreover, the concept of gene-environment interactions (GxE) was explored, which could provide novel insights into AD's pathophysiology. Finally, this chapter provided the aims and outline of this thesis.

Chapter 2: This chapter reviewed the existing literature on GxE research in $A D$, by exploring the impact of environmental exposure on the onset and progression of $A D$, both in epidemiological settings as well as in mouse models of $A D$. It was concluded that studying the effects of environmental exposures in humans is troubled by the long time-window between exposure and onset of $A D$ symptoms and the heterogeneity of the study population. These factors could be (partially) eliminated in mouse models of $A D$ as their controlled experimental setting allows us to study both the interactive and independent effects of genes and environment. However, the high degree of variability in research set-up in animal research made it challenging to draw firm and general conclusions about the impact of different environmental exposures on $A D$ onset and progression. Despite these methodological challenges, this chapter suggested that moving towards a GxE approach in $A D$ research may provide novel insights into the biological underpinnings of this disorder.

Chapter 3: As major depression has shown to be an independent risk factor for developing $A D$ later in life, this chapter explored the interaction between $A D$ and major depression. Its focus lied on the similarities between both illnesses in neuromorphological alterations and the (interactive) role of the serotonergic system and hypothalamic-pituitary-adrenal axis dysfunction in this respect.

Chapter 4: Here, the long-term effects of prenatal stress were explored on the progression of AD-related behavior and neuropathology in both male and female APPswe/PS1 $\triangle E 9$ offspring at 6 months of age. The results indicated highly sex-specific effects, with prenatally stressed males showing spatial memory deficits, while prenatally stressed female mice exhibit a relative improvement of spatial memory, but also more depressive-like behavior. In 
terms of neuropathological alterations it was demonstrated that female mice had a decreased hippocampal plaque load after prenatal stress, while male neuropathology remained unaltered. As prenatal perturbations are likely to mediate their effects via alterations in epigenetic regulation, the levels of various DNA methylation markers, such as DNA methyltransferase 3a, 5-methylcytosine and 5-hydroxymethylcytosine were also assessed, but appeared not to be alternatively regulated after prenatal stress exposure. In addition, several sex differences were observed independent of prenatal stress exposure, with female APPswe/PS1 $1 \mathrm{E} 9$ mice showing more spatial memory deficits and higher hippocampal plaque load than male mice of the same age. It was concluded that prenatal stress exposure impacted on the behavioral phenotype and neuropathology of APPswe/PS1 $1 \mathrm{E} 9$ mice in a sex-specific manner. Furthermore, it was suggested not to overlook the impact of sex-specific responses to environmental exposures when investigating gene-environment interactions in $A D$.

Chapter 5: Brain-derived neurotrophic factor (BDNF) has been postulated as a neurophysiological link between $A D$ and major depression. It was reported in chapter 4 that female APPswe/PS1 $\triangle E 9$ mice exposed to prenatal stress exhibited more depressive-like behavior. Considering the role of BDNF in depressive-like behavior and its interactions with amyloid- $\beta$, chapter 5 explored the effect of prenatal stress on the BDNF signaling pathway, including mature BDNF (mBDNF) proBDNF and their respective receptors, and the levels of soluble amyloid- $\beta$. Our research indicated that prenatal stress tended to lower mBDNF protein levels in the hippocampus, but only in female mice, who also demonstrated more depressive-like behavior. Moreover, APPswe/PS1 $1 \mathrm{E} 9$ female mice in general had higher levels of proBDNF and soluble amyloid- $\beta$ than their male counterparts. These findings suggested that BDNF signaling and amyloid production are sex-dependent, which may shed a new light on the selective vulnerability of women to develop both major depression and AD.

Chapter 6: In this chapter the efficacy of phosphodiesterase type 2 inhibitor (PDE2-I) BAY60-7550 was explored on the cognitive function and neuropathology of APPswe/PS1 $\triangle E 9$ mice. BAY60-7550 can prevent the break-down of second messengers cyclic AMP (cAMP) and cyclic GMP (cGMP), thereby stimulating intracellular signaling, the phosphorylation of cAMP response element-binding (CREB) protein and ultimately the formation of long-term potentiation; the proposed neurophysiological correlate of memory. The memory-enhancing effects of PDE2-I had already been demonstrated in adult and aged rodents, yet not in mouse models of $A D$. Chronic treatment of male APPswe/PS1 $\triangle E 9$ mice with BAY60-7550 resulted in improvements of spatial memory performance, without altering affective behavior. The underlying neurophysiological mechanisms of this memory improvement remain to be elucidated as no role for alterations in plaque load, levels of phosphorylated CREB, density of synaptophysin-immunoreactive presynaptic boutons (SIPBs) or BDNF levels in the hippocampus could be demonstrated. It was concluded that 
BAY60-7550 could be considered a promising therapeutic agent for the enhancement of memory, considering its ability to improve memory performance even after onset of pathology.

Chapter 7: The effects of chronic treatment of APPswe/PS1 $\triangle \mathrm{E} 9$ mice with the novel PDE4D-I GEBR-7b were investigated in this chapter. The results indicated that 3 weeks of GEBR-7b treatment at 5 months of age, could enhance long-term spatial memory performance in 7-month-old APPswe/PS1 $1 \mathrm{E} 9$ mice. To explore the underlying biochemical mechanisms of this memory improvement, concentrations of hippocampal CREB phosphorylation, BDNF, amyloid- $\beta$, tau, GSK3 $\beta$ and postsynaptic densities were investigated. No structural alterations in any of these markers could be demonstrated after 3 weeks of GEBR-7b treatment, despite the observed behavioral changes. It was concluded that PDE4D inhibition could be considered a novel therapeutic options in the treatment of $A D$ and other dementias, although the precise mechanisms of action remain to be defined.

Chapter 8: A history of antidepressant drug use can decrease the risk of developing AD and has shown to be beneficial for cognitive function in both major depression as well as $A D$ patients. To investigate how antidepressants can affect the development of $A D, 5$-month-old APPswe/PS1 $1 \mathrm{E} 9$ mice were treated with the selective serotonin reuptake inhibitor fluoxetine. Surprisingly, it was discovered that $45 \%$ of APPswe/PS1 1 E9 mice treated with fluoxetine displayed seizure behavior and that these mice were more likely to die prematurely. Although spontaneous behavioral seizures and EEG-recorded spontaneous epileptic discharges had been described before in this mouse model, the observation that this behavior could be induced by exogenous factors, such as SSRI treatment, was new. Fluoxetine has proconvulsive properties, yet is regularly prescribed to AD patients, who already have an increased susceptibility to develop epilepsy. This chapter suggested that one should be cautious when prescribing fluoxetine to AD patients and that its use should be monitored carefully.

Chapter 9: In the final chapter, all the findings of this thesis were summarized and discussed. It was concluded that chronic (developmental) stress exposure can permanently impact on the manifestation of AD-related symptoms and neuropathology. In addition, given the high degree of sex-specific effects, it was recommended that the effects of environmental exposure should be tested in both sexes as the impact may be profoundly different. Pharmacological intervention can have, depending on the agent, both protective as well as detrimental effects on AD progression. PDE inhibition could be considered a novel treatment strategy for $A D$, although more research will be needed to specify its mechanism of action and to elucidate its efficacy in the human population. Treatment with fluoxetine, however, should be considered with caution for AD patients. All in all, it was concluded that although many advances have been made in understanding the biological mechanisms underlying $A D$, many questions remain unanswered. 
Vereenvoudigde samenvatting 


\section{Achtergrond en doelstelling}

De ziekte van Alzheimer (AD) is een neurodegeneratieve ziekte, die momenteel 4.2 miljoen Europeanen treft en er wordt geschat dat dit aantal over de komende 20 jaar met $40 \%$ zal toenemen. Echter, de oorzaak van deze ziekte is nog altijd onbekend, wat de ontwikkeling van nieuwe medicijnen ernstig stagneert.

De symptomen van patienten met $A D$ zijn onder meer geheugenverlies, taalproblematiek, het onvermogen complexe handelingen uit te voeren, het onvermogen personen of voorwerpen te herkennen en problemen in zogeheten executieve functies (bijvoorbeeld plannen en organiseren). Om te komen tot een waarschijnlijkheidsdiagnose $A D$ moeten deze symptomen dusdanig ernstig zijn dat ze het sociale en/of professionele leven van de patient beperken. De ziekte wordt echter bovenal gekenmerkt door bepaalde veranderingen in de hersenen. De diagnose $A D$ kan dan ook pas met volledige zekerheid gesteld worden nadat er autopsie is verricht op de hersenen na overlijden en de aanwezigheid van deze hersenveranderingen, of neuropathologie, wordt vastgesteld.

De neuropathologie van $A D$ behelst een inkrimping van het hersenvolume als gevolg van het verlies van hersencellen, ofwel neuronen, en het verlies van contactpunten tussen neuronen, genaamd synapsen. Daarnaast wordt het gekenmerkt door de aanwezigheid van eiwitophopingen in en tussen de cellen, die de signaaloverdracht tussen neuronen bemoeilijken. Er zijn twee soorten eiwitophopingen: intracellulaire tangles bestaande uit het eiwit tau en extracellulaire plaques bestaande uit het eiwit amyloid- $\beta$ (A $\beta)$. Tau is normaal verantwoordelijk voor het stabiliseren van het celskelet, maar verandert bij $A D$ door nog onbekende redenen van vorm en kan daardoor niet langer deze functie uitoefenen. Het veranderde tau eiwit stapelt zich op en vormt kluwen binnen de cel, wat uiteindelijk resulteert in het afsterven van de cel. Het eiwit $A \beta$ wordt gevormd vanuit een groter eiwit genaamd amyloid precursor protein (APP), dat als gevolg van bepaalde enzymen (o.a. $\beta$-secretase en $Y$-secretase) wordt opgeknipt tot een kleiner fragment, genaamd $A \beta$. Hoewel dit proces bij gezonde mensen ook plaatsvindt, is er bij $A D$ patienten een overmaat in $A \beta$ productie en een tekort aan opruiming van dit eiwit, waardoor er grote hoeveelheden $A \beta$ aanwezig zijn in de hersenen. Zodra $A \beta$ zich buiten de cel bevindt, heeft het de neiging om zich op te hopen en dit leidt tot de aanwezigheid van $A \beta$ plaques in de hersenen van $A D$ patienten.

Genetisch onderzoek heeft ons geleerd welke genen betrokken zijn bij deze processen en dat mutaties in deze genen bij mensen kan leiden tot het onherroepelijk ontwikkelen van dementie. Bij AD zijn er mutaties gevonden in de genen voor APP, preseniline 1 (PS1) en preseniline 2 (PS2), allemaal betrokken bij productie van $A \beta$. Mensen met deze mutaties hebben de familiaire vorm van $A D$, maar vormen minder dan $2 \%$ van de $A D$ populatie. De overige $A D$ patienten hebben de zogenaamde sporadische vorm, waarbij de oorzaak meestal niet bekend is. Onderzoek naar de functie van deze AD genen heeft voor veel nieuwe inzichten in het ziekteproces gezorgd, maar kan het ontstaan van de sporadische gevallen helaas niet verklaren. Hierin is wellicht een rol voor de omgeving weggelegd. 
Het is bekend dat de omgeving een grote invloed kan hebben op het ontwikkelen van $A D$. Zo kan hoge blootstelling aan bepaalde metalen of pesticides en het oplopen van hersentrauma de kans op $A D$ vergroten, en hebben een mediterraans dieet en fysieke activiteit juist een risicoverminderend effect. Voor dit proefschrift is het feit dat stress en stress-gerelateerde stoornissen, zoals depressie, ook de kans op het ontwikkelen van AD kunnen verhogen, van bijzonder belang. Zo kan een geschiedenis van depressieve episodes de kans op $A D$ ontwikkeling tot twee keer toe verhogen, wat kan leiden tot een versnelde cognitieve achteruitgang en meer aanwezigheid van AD-gerelateerde pathologie in de hersenen, vergeleken met patienten zonder deze geschiedenis. In Hoofdstuk 2 van het proefschrift wordt uitgebreid aandacht besteedt aan de grote verscheidenheid aan omgevingsfactoren die bijdragen aan de kans op het ontwikkelen van AD.

Daarnaast wordt er in Hoofdstuk 2 ook aandacht geschonken aan een nieuw concept binnen het psychiatrisch onderzoek, namelijk gen-omgevingsinteracties. Het is duidelijk dat zowel genen als de omgeving individueel de kans op het ontwikkelen van AD kunnen beïnvloeden. Echter blijkt ook dat genen en omgeving kunnen interacteren om zo synergistisch te kunnen bijdragen aan de ziekteontwikkeling. Het bestuderen van deze genomgevingsinteracties (GxE), waarbij het effect van het een afhankelijk is van de aanwezigheid van het ander, zou nieuwe inzichten kunnen vergaren over het ontstaan van AD. Dit GxE concept vormt de basis van het onderzoek binnen dit proefschrift.

Om $A D$ te bestuderen maakt men gebruik van diermodellen, met name van muismodellen. Deze muismodellen zijn veelal gebaseerd op de mutaties in APP, PS1 en PS2 die bij mensen met familiaire AD zijn gevonden. Door het genoom van de muis te veranderen, en bijvoorbeeld deze menselijke $A D$ mutaties in te voegen, kunnen we een model voor $A D$ genereren waarbij de muis ook $A \beta$ plaques ontwikkelt en geheugenproblematiek laat zien. In dit proefschrift hebben wij gebruik gemaakt van het APPswe/PS1 1 E9 muis model, dat dus zowel een mutatie op APP als op PS1 bevat, waardoor het op jongere leeftijd (vanaf ongeveer 6 maanden oud) neuropathologie en geheugenverlies ontwikkelt.

Het hoofddoel van dit proefschrift was te bestuderen hoe veranderingen in de omgeving kunnen bijdragen aan het ontstaan en de ontwikkeling van AD. Specifieker nog, wij hebben onderzocht hoe externe factoren, zoals blootstelling aan chronische stress en farmacologische middelen, de manifestatie van de ziekte zou beïnvloeden. Als uitkomstmaten hebben wij gekeken naar veranderingen in gedrag en de neuropathologie.

Dit proefschrift is opgedeeld in twee delen, waarbij het eerste deel zich richt op stress als risicofactor voor $A D$ en in het tweede deel de effecten van farmacologische middelen op de progressie van $A D$ worden beschreven. 


\section{Deel 1: Stress als risicofactor voor AD}

Deel 1 van dit proefschrift behandelt welke effecten de chronische blootstelling aan stress heeft op het ontstaan en de ontwikkeling van AD-gerelateerde cognitieve achteruitgang en neuropathologie. Aangezien onderzoek heeft aangetoond dat depressie, een veelvoorkomend stress-gerelateerd ziektebeeld, een risicofactor kan zijn voor het ontwikkelen van $A D$, wordt deze ziekte-interactie verder uitgediept in Hoofdstuk 3. Hierin worden de gelijkenissen beschreven tussen de twee ziektebeelden op het gebied van hersenveranderingen en welke (interactieve) rol het serotonerge systeem (een neurotransmitter, of signaalmolecuul in de hersenen) en de dysfunctie van de hypothalamus-hypofyse-bijnier-as (betrokken bij de regulatie van stresshormoonafgifte in het lijf) hierin spelen.

In Hoofdstuk 4 en $\mathbf{5}$ worden de empirische experimenten van dit proefschrift beschreven waarin is onderzocht hoe stress tijdens de zwangerschap kan bijdragen aan het ontwikkelen van $A D$ op de lange termijn. Het is bekend dat verstoringen tijdens de prenatale periode een kritieke invloed kunnen hebben het ontwikkelen van psychiatrische ziektebeelden bij het nageslacht, waaronder depressie. Dit wordt waarschijnlijk bewerkstelligd door veranderingen in epigenetische controle van gen-expressie. Bij het vertalen van genen naar functionele eiwitten kan de mate van deze vertaling beïnvloed worden door epigenetische processen als DNA methylering (het toevoegen van een methylgroep aan DNA moleculen, waardoor het DNA minder goed valt af te lezen). Epigenetische processen zijn van essentieel belang in de normale ontwikkeling van de foetus maar waarschijnlijk ook in het mediëren van het ontwikkelen van ziekte of gezondheid op volwassen leeftijd. Er kan daarom verondersteld worden dat verstoringen tijdens de zwangerschap via epigenetische regulatie langdurige effecten kunnen hebben op de ontwikkeling van AD-gerelateerd gedrag en neuropathologie op latere leeftijd.

Vanuit dit concept werd er stress toegebracht aan zwangere muizen, door hen op meerdere tijdstippen per dag in hun bewegingsruimte te beperken gedurende de eerste week van de zwangerschap. Op basis van eerdere studies met chronische stress tijdens de puberteit en de volwassenheid werd er gehypothetiseerd dat ook deze vorm van stress zou leiden tot een versnelling van de cognitieve achteruitgang en verhoogde expressie van ADgerelateerde neuropathologie en dat dit gemediëerd zou worden via veranderingen in epigenetische regulatie. Deze effecten werden bekeken in zowel mannelijk als vrouwelijk APPswe/PS1 $1 \triangle$ E nageslacht.

Onze data toonde aan dat prenatale stress (PS) sterke geslachtsafhankelijke effecten veroorzaakt. Mannelijke APPswe/PS1 $\triangle E 9$ muizen die PS hebben gehad vertoonden met name cognitieve problematiek, zoals verminderd ruimtelijk geheugen, en een verstoorde hypothalamus-hypofyse-bijnier-as functie. Echter, de neuropathologie en de regulatie van brain-derived neurotrophic factor (BDNF, een zenuwstelstimulerend eiwit dat zowel cognitieve als emotionele processen kan beïnvloeden) bleef onveranderd na PS in mannelijke APPswe/PS1 $\triangle E 9$ muizen. Daarentegen duidden onze resultaten aan dat in 
vrouwelijke APPswe/PS1 $\triangle \mathrm{E} 9$ muizen PS juist leidde tot meer depressief-achtig gedrag en een relatieve bescherming tegen cognitieve achteruitgang. In lijn met dit laatste vertoonden PS vrouwtjes een onverwachte vermindering in de hoeveelheid $A \beta$ plaques in de hippocampus, een structuur die betrokken is bij leer- en geheugenprocess. Daarnaast hadden deze vrouwtjes een verstoring in hun BDNF signalering, een bevinding die ook gezien wordt bij patienten met depressie. De hypothese dat hervormingen in epigenetische regulatie ten grondslag zouden liggen aan deze gedragsveranderingen kon niet bevestigd worden door onze resultaten.

Naast deze sekse-afhankelijke effecten van PS lieten onze data ook zien dat er in het algemeen al grote sekse-verschillen bestaan in dit muismodel. Vrouwelijke APPswe/PS1 $1 \mathrm{E} 9$ muizen hebben in vergelijk met mannelijke muizen van dezelfde leeftijd meer cognitieve problemen en meer neuropathologie. Geslachtshormonen spelen mogelijk een rol bij deze verschillen.

\section{Deel 2: Effecten van farmacologische interventie}

In deel 2 zijn de effecten op gedrag en neuropathologie onderzocht van twee typen farmacologische middelen, namelijk phosphodiesterase (PDE) remmers en het antidepressivum fluoxetine.

\section{PDE remmers}

Zowel acute als chronische behandeling met PDE remmers heeft in het verleden aangetoond dat deze middelen geheugenverbeterende effecten hebben. Maar wat is nu de werking van deze middelen? Zodra een neuron input krijgt, leidt dit tot een aaneenschakeling van intracellulaire signalen door activering van signaalmoleculen. De nucleotiden cAMP en cGMP zijn hier twee voorbeelden van die aan het begin van deze signaalcascade staan. Hun activatie kan uiteindelijke leiden tot het activeren van genexpressie en de afgifte van nieuw gevormde eiwitten, waarmee vele intracellulaire processes beïnvloed kunnen worden. Een van die processes is het verbeteren van de signaaloverdracht tussen twee hersencellen, iets wat lange-termijn potentiatie wordt genoemd en waarvan gedacht wordt dat dit het cellulaire substraat is van geheugen. Onder normale omstandigheden worden de nucleotiden CAMP en CGMP snel afgebroken door PDE's, een proces dat door de PDE remmers tegengegaan kan worden. Toediening van PDE remmers kan dus uiteindelijke leiden tot een verhoogde activatie van de genoemde intracellulaire signaalcascades en een grotere kans op het totstandkomen van het cellulaire geheugen.

Er zijn vele types en subtypes PDE's, waarvan een aantal specifiek tot expressie komen in die hersengebieden die betrokken zijn bij leer- en geheugenprocessen. Door het gebruik van twee specifieke PDE remmers, namelijk een PDE2 en een PDE4D remmer, hebben wij onderzocht in hoeverre deze farmacologische middelen ook in staat zouden zijn tot 
geheugenverbetering in een model voor $A D$. De resulten hiervan staan beschreven in Hoofdstuk 6 en 7.

Ons onderzoek toonde aan dat chronische behandeling met zowel de PDE2 remmer als de PDE4D remmer kan leiden tot geheugenverbetering in APPswe/PS1 $\triangle E 9$ muizen. De bevinding dat deze effecten ook bereikt kunnen worden na het intreden van neuropathologie in de muizen, toonde aan dat deze middelen ook geschikt zouden zijn voor AD patienten. Patienten worden immers ook pas behandeld nadat de diagnose AD wordt gesteld, terwijl de neuropathologie dan al in grote mate aanwezig is. Via welke mechanismes de geheugenverbeterende effecten worden bewerkstelligd, dient in toekomstig onderzoek verder te worden onderzocht, aangezien onze resultaten daar op dit moment geen uitsluitsel over geven.

\section{Fluoxetine}

Veel AD patienten ontvangen naast hun cognitieverbeterende medicijnen ook antidepressiva om het hoofd te bieden aan de gedragsmatige en psychologische symptomen geassocieerd met dementie, waaronder aggressie, apathie en depressie. In Hoofdstuk 8 hebben wij onderzocht in hoeverre de chronische toediening (21 dagen) van het antidepressivum fluoxetine, een selective serotonine heropname remmer (SSRI), een positief effect zou hebben op AD-gerelateerde geheugenverslechtering en neuropathologie in APPswe/PS1 $\triangle \mathrm{E} 9$ muizen. Verrassend genoeg bleek dat bijna de helft van de APPswe/PS1 $\triangle E 9$ dieren behandeld met fluoxetine gedrag ontwikkelde dat sterk leek op epileptische aanvallen. Daarnaast werd er binnen dezelfde groep een bovengemiddelde sterftegraad geobserveerd. Na 17 dagen werd omwille van ethische redenen besloten de behandeling van deze dieren vroegtijdig te staken. Statistische analyse toonde aan dat zowel het APPswe/PS1 $1 \mathrm{E} 9$ genotype als fluoxetine behandeling significante voorspellers waren voor het ontwikkelen van epileptische aanvallen. Daarnaast bleek het hebben van deze aanvallen, het APPswe/PS1 $\triangle \mathrm{E} 9$ genotype en de fluoxetine behandeling het vroegtijdig overlijden van de muizen significant te kunnen voorspellen.

Uit de literatuur bleken veel aanwijzingen te komen die deze data kunnen verklaren. Zo zijn epileptisch gedrag en epileptische hersenactiviteit al eerder gerapporteerd voor dit muismodel, maar bleek onze bevinding dat het geïnduceerd kon worden door een externe factor de eerste in zijn soort te zijn. Daarnaast duidde de literatuur aan dat fluoxetine zowel anti- als pro-convulsief kan werken, afhankelijk van de dosering. Ook werd duidelijk dat fluoxetine zich makkelijk in het lichaam kan opstapelen waardoor er, wellicht in combinatie met een veranderd metabolisme in APPswe/PS1 $\triangle E 9$ muizen, hoge concentraties zouden hebben kunnen ontstaan die mogelijk de epileptische aanval hebben opgewekt.

Men moet zich realiseren dat $A D$ patienten een verhoogd risico hebben op het ontwikkelen van epilepsie. Naar onze mening is voorzichtigheid dan ook geboden wanneer men fluoxetine voorschrijft aan $A D$ patienten, omdat de proconvulsieve eigenschappen van 
dit middel in combinatie met de epileptische gevoeligheid van deze patientgroep de ontwikkeling van epileptische insulten zouden kunnen opwekken.

\section{Algemene conclusie}

In het algemeen kan worden geconcludeerd dat veranderingen in de omgeving daadwerkelijk de expressie van AD-gerelateerde cognitieve achteruitgang en neuropathologie kunnen beïnvloeden. Echter heeft ons onderzoek aangetoond dat deze effecten sterk geslachtsafhankelijk kunnen zijn. Men dient met deze bevinden rekenschap te houden wanneer men onderzoek doet naar effecten van omgevingsfactoren in muismodellen van $A D$, aangezien dit van grote invloed kan zijn op de uitkomstmaten. Daarnaast laat ons onderzoek zien dat veranderingen in het gedrag niet altijd gepaard gaan met veranderingen in neuropathologie, wat nogmaals de complexiteit benadrukt van de hersenen en de manier waarop ze hun functies uitoefenen. Ondanks de noodzaak voor aanvullend onderzoek om de onderliggende mechanismes te ontdekken, kan er gesteld worden dat PDE remmers een nieuw middel zouden kunnen worden in de behandeling van $A D$. Daarentegen zou het gebruik van fluoxetine door $A D$ patienten op zijn minst goed gemonitord moeten worden.

In 1906 werd door Alois Alzheimer AD voor het eerst herkend als een afzonderlijke psychiatrische ziekte met interessante hersenveranderingen. $\mathrm{Nu}$, meer dan 100 jaar na zijn eerste ontdekking, hebben wij vele nieuwe inzichten vergaard in de ziekteprocessen die een rol spelen bij het onstaan van deze ziekte. Laten wij er naar streven dat het niet nog eens 100 jaar zal duren voordat de vele onopgeloste vragen, die uiteindelijk zullen leiden tot het vinden van een geneesmiddel voor dit fascinerende ziektebeeld, beantwoord kunnen worden. 


\section{Appendices}

About the Author

List of Publications

Affiliations of co-authors 


\section{About the author}

Susanne Rianneke (Annerieke) Sierksma was born on February $10^{\text {th }}, 1984$ in Zwolle (the Netherlands). She attended the Marnix College in Ede, where she received a bilingual Dutch-English education ('tweetalig VWO') and completed the International Baccalaureate English A2 program. At the age of 16, she attended the American high school Cape Henry Private Collegiate at Virginia Beach, VG, USA, for 2 weeks and the year after she participated in the Global Young Leaders Conference in Washington and New York.

In 2002, she relocated to Maastricht to start the Bachelor Biological Psychology at Maastricht University. She was given the opportunity to take her elective courses at Bond University in Robina, Queensland, Australia in 2005, which resulted in an 8-month visit to study and travel. In 2005, she commenced her Master Neuropsychology at Maastricht University, while receiving her Bachelor of Science degree in July 2006. The mandatory research internship of her Master's degree was performed at the School for Mental Health and Neuroscience (MHeNS) of Maastricht University, under supervision of Christoph Schmitz. During this internship, Annerieke investigated neuron and interneuron numbers in the frontal cortex of mouse models of Alzheimer's disease (AD). Subsequently, she was offered a PhD position within the same group, which started in September 2007. After 1 year of research on morphological alterations in mouse models of $A D$, her initial PhD supervisor, Christoph Schmitz, left Maastricht University and the project was terminated.

Annerieke was offered a new project within MHeNS under supervision of Dr. Jos Prickaerts, Dr. Daniel van den Hove and Prof. Dr. Harry Steinbusch, the work of which culminated into this doctoral thesis. During her time in Maastricht she was PhDrepresentative of division 3 at MHeNS and was co-founder and chair of the choir 'Musicalkoor Maastricht'. Annerieke continues to research Alzheimer's disease as a postdoctoral fellow at the Laboratory for the Research of Neurodegenerative Diseases at the KU Leuven (Belgium) under supervision of Prof. Bart De Strooper and is currently living in Brussels. 


\section{List of publications}

\section{Peer-reviewed international publications:}

ASR Sierksma, K Rutten, S Sydlik, S Rostamian, HWM Steinbusch, DLA van den Hove, J Prickaerts (2012). Chronic phosphodiesterase type 2 inhibition improves memory in the APPswe/PS1 1 E9 mouse model of Alzheimer's disease. Neuropharmacology, 64(1), p.12436.

ASR Sierksma, T Vanmierlo, J De Vry, ME Raijmakers, HWM Steinbusch, DLA van den Hove, J Prickaerts (2012). Effects of prenatal stress exposure on soluble $A \beta$ and brainderived neurotrophic factor signaling in male and female APPswe/PS1 $\triangle E 9$ mice. Neurochemistry International, 61(5), p. 697-701.

ASR Sierksma, J Prickaerts, L Chouliaras, S Rostamian, L Delbroek, BPF Rutten, HWM Steinbusch, DLA van den Hove (2012). Behavioral and neurobiological effects of prenatal stress exposure in male and female APPswe/PS1 $1 E 9$ mice. Neurobiology of Aging, 34(1), p. 319-37.

MAM Lemmens, ASR Sierksma, BPF Rutten, F Denissen, HWM Steinbusch, PJ Lucassen, C Schmitz (2011). Age-related changes of neuron numbers in the frontal cortex of a transgenic mouse model of Alzheimer's disease. Brain Structure and Function, 216(3), p. 227-37.

ASR Sierksma*, L Chouliaras*, G Kenis, J Prickaerts, MAM Lemmens, I Brasnjevic, EL van Donkelaar, P Martinez-Martinez, M Losen, MH De Baets, N Kholod, FW van Leeuwen, PR Hof, J van Os, HWM Steinbusch, DLA van den Hove, BPF Rutten (2010; * joint first authors). Gene-environment interaction research and transgenic mouse models of Alzheimer's disease. International Journal of Alzheimer's disease (epub ahead of print). DOI: $10.4061 / 2010 / 859101$

ASR Sierksma, DLA van den Hove, HWM Steinbusch, J Prickaerts (2010). Major depression, cognitive dysfunction and Alzheimer's disease: Is there a link? European Journal of Pharmacology, 626(1), p. 72-82. 


\section{Manuscripts in preparation:}

MAM Lemmens, ASR Sierksma, K Heck, HWM Steinbusch, BPF Rutten, C Schmitz (2012). Age-related changes synaptophysin immunoreactive presynaptic boutons in the frontal cortex of a transgenic mouse model of Alzheimer's disease. In preparation

ASR Sierksma, DLA van den Hove, T Vanmierlo, F Pfau, M Philippens, O Bruno, E Fedele, R Ricciarelli, HWM Steinbusch, J Prickaerts. Improvement of spatial memory function in $A P P s w e / P S 1 \triangle E 9$ mice after chronic inhibition of phosphodiesterase type $4 D$. In preparation

ASR Sierksma, J Prickaerts, G Hoogland, T Vanmierlo, FW van Leeuwen, HWM Steinbusch, DLA van den Hove. Increased mortality in APPswe/PS1 $1 E 9$ mice after chronic fluoxetine treatment: a role for epileptic seizures? In preparation

\section{Book Chapter (in Dutch):}

HMW Steinbusch, ASR Sierksma, C Schmitz (2007). Neurobiologie van hersenveroudering. In: WG Van Aken, KR Ridderinkhof, FRJ Verhey, M Everblij (Eds.), 'Vergrijzend Brein - wat ouder wordende hersenen met cognitieve vermogens doen' (p.18-23). Kwartaalcahier van de Stichting Bio-Wetenschappen en Maatschappij. Leiden, Nederland, Drukkerij Groen BV.

\section{Abstracts:}

ASR Sierksma, MAM Lemmens, P van Tijn, SRM Philtjens, HWM Steinbusch, DF Fischer, C Schmitz, FW van Leeuwen (2008). $U B B^{+1}$ could be protective for APPswe/PS1 $\triangle E 9$ induced striatal neurodegeneration in a novel mouse model for Alzheimer's disease.

- MHeNS Research Day 2008, Maastricht, the Netherlands (poster presentation)

ASR Sierksma, J Prickaerts, L Chouliaras, K Rutten, HWM Steinbusch, DLA van den Hove (2009). Prenatal maternal stress accelerates cognitive decline in the APPswe/PS1 1 E9 mouse model of Alzheimer's disease.

- Euron PhD Days 2009, Nijmegen, the Netherlands (poster presentation)

- MHeNS Research Day 2009, Maastricht, the Netherlands (poster presentation)

ASR Sierksma, DLA van den Hove, GRL Kenis, HWM Steinbusch, J Prickaerts, K Rutten (2009). Phosphodiesterase type 2 inhibitor BAY 60-7550 ameliorates hippocampusdependent memory in the APPswe/PS1 $\triangle E 9$ mouse model of Alzheimer's disease.

- 8th Endo-Neuro-Psycho Meeting, Doorwerth, the Netherlands (poster presentation) 
ASR Sierksma, J Prickaerts, L Delbroek, L Chouliaras, K Rutten, HWM Steinbusch, DLA van den Hove (2010). Prenatal maternal stress sex-dependently alters memory and affect in the APPswe/PS1 1 E9 mouse model of Alzheimer's disease.

- MHeNS Research Day, Maastricht, Netherlands (poster presentation)

- Euron Workshop 'Drugs and the brain: an update on psychopharmacology', Braga, Portugal (poster presentation)

- ISAO/AHAF Workshop 'Update on Alzheimer research', San Diego, CA, USA (poster presentation)

- Society for Neuroscience Annual Meeting, San Diego, CA, USA (poster presentation)

ASR Sierksma, J Prickaerts, L Chouliaras, L Delbroek, S Rostamian, K Rutten, HWM Steinbusch, DLA van den Hove (2011). Prenatal maternal stress sex-dependently alters spatial memory, depressive-like behaviour and plaque load in the APPswe/PS1 model of Alzheimer's disease.

- 9th Endo-Neuro-Psycho Meeting, Lunteren, the Netherlands (poster presentation)

- MHeNS Research Day, Maastricht, The Netherlands (poster presentation)

- Euron PhD Days, Bonn, Germany (oral presentation)

ASR Sierksma, DLA van den Hove, K Rutten, L Chouliaras, S Rostamian, HWM Steinbusch, J Prickaerts (2011). Chronic phosphodiesterase type 2 inhibition improves spatial memory and alters synaptic density in the hippocampus in the APPswe/PS1 1 E9 mouse model of Alzheimer's disease

- Society for Neuroscience Annual Meeting, Washington DC, USA (poster presentation) 


\section{Affiliations co-authors}

Brasnjevic, I

Bruno, O

Chouliaras, L

Delbroek, L

De Baets, $\mathrm{MH}$

De Vry, J

Fedele, $\mathrm{E}$

Hof, PR
Department of Psychiatry and Neuropsychology, Faculty of Health, Medicine and Life Science, School for Mental Health and Neuroscience, Maastricht University, Maastricht, the Netherlands

Department of Pharmaceutical Sciences, Section of Pharmacology and Toxicology, University of Genoa, Genoa, Italy

Department of Psychiatry and Neuropsychology, Faculty of Health, Medicine and Life Science, School for Mental Health and Neuroscience, Maastricht University, Maastricht, the Netherlands

Department of Psychiatry and Neuropsychology, Faculty of Health, Medicine and Life Science, School for Mental Health and Neuroscience, Maastricht University, Maastricht, the Netherlands

Department of Psychiatry and Neuropsychology, Faculty of Health, Medicine and Life Science, School for Mental Health and Neuroscience, Maastricht University, Maastricht, the Netherlands

School of Life Sciences, Biomedical Research Institute and Translationale Universiteit Limburg, Hasselt University, Belgium

Department of Psychiatry and Neuropsychology, Faculty of Health, Medicine and Life Science, School for Mental Health and Neuroscience, Maastricht University, Maastricht, the Netherlands

Department of Experimental Medicine, Section of Pharmacology and Toxicology, University of Genoa, Genoa, Italy

Department of Neuroscience, Mount Sinai School of Medicine, New York, NY, USA 
Hoogland, G

Kenis, G

Kholod, N

Lemmens, MAM

Losen, M

Lucassen, $\mathrm{P}$

Martinez-Martinez, $\mathrm{P}$

Pfau, F

Philippens, M
Department of Psychiatry and Neuropsychology, Faculty of Health, Medicine and Life Science, School for Mental Health and Neuroscience, Maastricht University, Maastricht, the Netherlands

Department of Neurosurgery, Maastricht University Medical Center, Maastricht, the Netherlands

Department of Psychiatry and Neuropsychology, Faculty of Health, Medicine and Life Science, School for Mental Health and Neuroscience, Maastricht University, Maastricht, the Netherlands

Department of Psychiatry and Neuropsychology, Faculty of Health, Medicine and Life Science, School for Mental Health and Neuroscience, Maastricht University, Maastricht, the Netherlands

Department of Psychiatry and Neuropsychology, Faculty of Health, Medicine and Life Science, School for Mental Health and Neuroscience, Maastricht University, Maastricht, the Netherlands

Department of Psychiatry and Neuropsychology, Faculty of Health, Medicine and Life Science, School for Mental Health and Neuroscience, Maastricht University, Maastricht, the Netherlands

Center for Neuroscience, Swammerdam Institute for Life Sciences, University of Amsterdam, Amsterdam, the Netherlands

Department of Psychiatry and Neuropsychology, Faculty of Health, Medicine and Life Science, School for Mental Health and Neuroscience, Maastricht University, Maastricht, the Netherlands

Department of Psychiatry and Neuropsychology, Faculty of Health, Medicine and Life Science, School for Mental Health and Neuroscience, Maastricht University, Maastricht, the Netherlands

Department of Psychiatry and Neuropsychology, Faculty of Health, Medicine and Life Science, School for Mental Health and Neuroscience, Maastricht University, Maastricht, the Netherlands 
Prickaerts, $\mathrm{J}$

Raijmakers, MEA

Ricciarelli, $\mathrm{R}$

Rostamian, S

Rutten, BPF

Rutten, K

Steinbusch, HWM

Sydlik, S

Vanmierlo, $\mathrm{T}$

Van den Hove, DLA
Department of Psychiatry and Neuropsychology, Faculty of Health, Medicine and Life Science, School for Mental Health and Neuroscience, Maastricht University, Maastricht, the Netherlands

Department of Psychiatry and Neuropsychology, Faculty of Health, Medicine and Life Science, School for Mental Health and Neuroscience, Maastricht University, Maastricht, the Netherlands

Department of Experimental Medicine, Section of General Pathology, University of Genoa, Genoa, Italy

Department of Psychiatry and Neuropsychology, Faculty of Health, Medicine and Life Science, School for Mental Health and Neuroscience, Maastricht University, Maastricht, the Netherlands

Department of Psychiatry and Neuropsychology, Faculty of Health, Medicine and Life Science, School for Mental Health and Neuroscience, Maastricht University, Maastricht, the Netherlands

Department of Psychiatry and Neuropsychology, Faculty of Health, Medicine and Life Science, School for Mental Health and Neuroscience, Maastricht University, Maastricht, the Netherlands

Department of Psychiatry and Neuropsychology, Faculty of Health, Medicine and Life Science, School for Mental Health and Neuroscience, Maastricht University, Maastricht, the Netherlands

Department of Psychiatry and Neuropsychology, Faculty of Health, Medicine and Life Science, School for Mental Health and Neuroscience, Maastricht University, Maastricht, the Netherlands

Department of Psychiatry and Neuropsychology, Faculty of Health, Medicine and Life Science, School for Mental Health and Neuroscience, Maastricht University, Maastricht, the Netherlands

Department of Psychiatry and Neuropsychology, Faculty of Health, Medicine and Life Science, School for Mental 
Van Donkelaar, EL

Van Leeuwen, FW

Van Os, J
Health and Neuroscience, Maastricht University, Maastricht, the Netherlands

Department of Psychiatry, Psychosomatics and Psychotherapy, University of Würzburg, Würzburg, Germany

Department of Psychiatry and Neuropsychology, Faculty of Health, Medicine and Life Science, School for Mental Health and Neuroscience, Maastricht University, Maastricht, the Netherlands

Department of Psychiatry and Neuropsychology, Faculty of Health, Medicine and Life Science, School for Mental Health and Neuroscience, Maastricht University, Maastricht, the Netherlands

Department of Psychiatry and Neuropsychology, Faculty of Health, Medicine and Life Science, School for Mental Health and Neuroscience, Maastricht University, Maastricht, the Netherlands 
Waarschijnlijk het best gelezen onderdeel van elk proefschrift. In dit stuk zou ik graag de gelegenheid nemen om iedereen te bedanken, die op een of andere wijze heeft bijgedragen aan de totstandkoming van dit proefschrift.

Allereerst wil ik mijn promoter Prof. Dr. Harry Steinbusch bedanken voor de mogelijkheid om binnen de School for Mental Health and Neurosicence te promoveren en voor de interesse in mijn voortgang en verdere wetenschappelijke carriere. Ook hartelijk dank voor al uw hulp en inzet om zaken als de MHeNS Mingle Day en de Pizzameeting te realiseren.

Daarnaast wil ik graag mijn 2 copromotoren, Dr. Jos Prickaerts en Dr. Daniel van den Hove, bedanken voor de dagelijkse begeleiding. Ik heb het zeer gewaardeerd dat jullie mij na het vertrek van Christoph in jullie groep(en) hebben opgenomen, en mij het vertrouwen gaven om aan jullie project te werken. Wat heb ik veel van jullie geleerd!

Beste Jos: jouw loyaliteit naar de leden binnen onze groep is bewonderingswaardig, en ik heb zeer veel waardering voor alle inzet die je aan mijn project hebt gewijd, zowel in tijd, energie als op het financiele vlak. Bedankt voor het overdragen van je kennis aan psychopharmacology en gedrag. Meten is weten!

Beste Daniel: jij natuurlijk ook heel hartelijk bedankt voor alle toewijding waarmee je mij hebt begeleid de afgelopen jaren. Je bent een didact in hart en nieren, en je gevleugelde uitspraken als, 'It goes from a slate roof', 'Science is fun' en mijn persoonlijk favoriet 'Zonder wrijving geen glans', zullen me nog lang bij blijven.

I would also like to express my gratitude to my thesis evaluation committee for reading yet another $\mathrm{PhD}$ thesis, despite their busy schedules. Also many thanks in advance to the members of the corona for their time to attend my defense and read my thesis.

Mijn paranimfen Sven Akkerman en Eva Bollen: ik vind het heel bijzonder dat jullie de 'grote dag' samen met mij meemaken, zoals jullie ook de rest van mijn PhD-tijd hebben meegemaakt: up close and personal :-) Bedankt voor jullie al steun, hulp en interesse tijdens dit traject, maar met name bedankt voor alle lol en gezelligheid op onze kamer en op congressen (ENP!!)! Ik wens jullie het allerbeste voor jullie eigen promotie!

Of course, I would also like to thank the plethora of fellow PhD-students (or young doctors) at MHeNS for making my time at Maastricht University so wonderful. There were so many over the past years (or was I just there for a long time?), so I'm bound to forget a few names. Some of them I would like to thank in person.

De 'In vivo groep' olv Jos: Eva Bollen, Sven Akkerman, Jochen De Vry, Tim Vanmierlo, Nick van Goethem, Olga Reneerkens, Marlies van Duinen, en ons 'adoptiekind' Rianne Opstelten. Bedankt voor alle gezellige dagen op kantoor met een overvloed aan bakjes Senseo, practical jokes, klaagsessies, barbecues/thuis-bioscoop-avonden, in-vivo-uitjes en avondjes in de Fallstaff/Alla. Maar natuurlijk ook voor de wetenschappelijke discussies en 
alle hulp op het lab en met de dieren. Jullie zijn allemaal goud waard en ik wens jullie al het beste voor de toekomst!

Leonidas Chouliaras: thanks for introducing me into the fascinating world of epigenetics and the good times at conferences and workshops. I'm sure you'll be a great psychiatrist some day and wish you all the best for the future.

De 'meisjes' van de afdeling (back in the day): Marijke Lemmens, Eveline Strackx, Imke van Kooten, Evi Lemmens, Kim Rijkers en Rinske Vlamings. Bedankt voor jullie warme ontvangst toen ik net bij MHeNS kwam werken (en aan Marijke en Imke voor de PhD-plek ;-) ) en de gezellige etentjes die volgden, ook nadat iedereen al gepromoveerd was. ledereen het allerbeste gewenst met de gezinsuitbreidingen, huizen die gebouwd moeten worden, en overige carriere-stappen! In het bijzonder wil ik Marijke bedanken voor alle uren die we samen hebben doorgebracht. Ik heb veel van je mogen leren, zowel op het gebied van onderzoek als universitaire politiek, maar heb met name mogen genieten van je Vlaamse gezelligheid en je toewijding aan je dierbaren. Ik heb veel bewondering voor hoe je je overal door heen hebt geworsteld en wens je alle geluk voor de toekomst.

Many of the other PhD students: Mark Janssen, Dagmar Zeef, Youssef Yakkiou, Sonny Tan, Thibault Sesia, Bart Biallosterski, Pablo Andrade, Evi Vlassaks, Kathleen Vrolix, Caroline Hammels, Ali Jahanshahianvar, Ehsan Pishva, Fabien Boullé, Marisela Martinez-Claros, and all the ones I am forgetting: we really had a great group of like-minded PhD students in the lab. Thanks for everything, particularly all the great Euron PhD days (or should I say nights?)!

Ik wilde ook nog de eerste lichting MHeNS-vertegenwoordigers (of 'AIO-meisjes') bedanken: Rinske, Deliane, Rosa, Margreet, Rebecca, Gisela. Ik heb ontzettend genoten van onze bijeenkomsten om zaken als de MHeNS Mingle Day en de Pizzameetings te organiseren, en de gezellige etentjes die daar meestal aan voorafgingen. Bedankt voor deze verrijking van mijn AIO-tijd!

Natuurlijk ook hartelijk dank aan de overige collega's, waarvan ik er wederom een paar bij naam zal noemen:

Gunther Kenis: bedankt voor je al je wetenschappelijk hulp en inzicht, maar ook voor je virtuositeit op de piano! Stille wateren, hebben diepe gronden...

Kris Rutten: bedankt voor de mogelijkheid om de PDE2 studie te kunnen uitvoeren, en voor je hulp en kritische blik bij de uitvoering en afronding daarvan. Al het beste voor jou en je gezin!

Eva van Donkelaar: wat een mazzel dat ik jou nog heb mogen ontdekken in het laatste jaar van jouw PhD en je post-doc-tijd op onze afdeling! Je humor en Hollandse nuchterheid zorgden voor een gouden randje aan mijn tijd op de afdeling en ik heb met veel plezier met je samengewerkt op die ver-\#\$\%* APPswe/PS1 $\triangle E 9$ muizen die altijd met bosjes neervielen. 
De analisten: Hellen Steinbusch, Denise Hermes, Marjan Philippens en Marjanne Markerink. Heel hartelijk dank voor jullie technische ondersteuning in het lab en de lab-vaardigheden die ik van jullie heb mogen leren!

Ik zou ook graag de voorgaande en huidige secretaresses willen bedanken, in het bijzonder Marie-Thérèse Moers en Lisa Pizzuto, voor alle administratieve ondersteuning. Daarnaast ook dank aan Anja de Bruin en Nicole Senden voor de fantastische organisatie van de MHeNS PhD dagen, Euron Workshops, en al het werk achter de schermen waarvan wij niet op de hoogte zijn.

Daarnaast verdienen ook de mensen van het CPV mijn dank, met in het bijzonder Richard Frijns, Inger van Rijn en Rik Tinnemans, voor al hun ondersteuning bij mijn experimenten.

I would also like to thank my first $\mathrm{PhD}$ supervisor, Christoph Schmitz. Even though our collaboration was brief, I can say I became richer from the experience. Thank you for offering me the initial opportunity to start my $\mathrm{PhD}$ at MHeNS.

I could not have accomplished all this work, without the help of many Bachelor and Master students: Lore Delbroek, Somayeh Rostamian, Sebastian Sydlik, Mathias Leinders, Marjolein Raijmakers, Franziska Pfau. I have learned a great deal from supervising you. I hope you can say the same ;-)

Daarnaast wil ik graag de leden van Musicalkoor Maastricht bedanken voor de vaste gezelligheid op de woensdagavond, met in het bijzonder Florence, Jess, Corien, Maarten, Manuela, Jacqueline en Octave. Wie had ooit gedacht dat zo'n samengeraapt zooitje musicalliefhebbers het zo fantastisch zou hebben samen?

Natuurlijk kan hier ook niet Jaar 2002 ontbreken: Anneloes, Meike, Melisse, Tessa, Marieke, Hannah en Yvette. Onze fantastische weekendjes weg, ski-tripjes en dagjes Amsterdam zorgden geregeld voor de broodnodige afleiding en oplading om weer door te ploeteren. Bedankt voor jullie steun van een afstand: jullie zijn me erg dierbaar!

Mijn vriendinnetjes van thuis, Alicia, Amber en Stefanie: ondanks dat onze levens steeds meer veranderen, kan ik me altijd verbazen over hoe makkelijk we de draad weer oppakken, en het weer vanouds gezellig is. Bedankt voor alles!

Ook wil ik graag mijn familie bedanken voor hun interesse, ellenlange geduld met mijn 'muizenverhalen' en steun als het even tegenzat. Bij jullie voel ik me altijd 'thuis', iets waarvan ik heb geleerd dat het niet vanzelfsprekend is. Jullie warmte en liefde waardeer ik enorm en ik hoop op een voorspoedige en gelukkige toekomst voor ons allemaal.

In bijzonder wil ik mijn moeder bedanken, die er altijd voor me is geweest door dik en dun, op persoonlijk alswel op professioneel vlak. Bedankt voor al je advies en je gezelschap. Jouw kracht is onevenaarbaar. 
Mijn liefste Patrick, de laatste regels zijn voor jou. 'Absence makes the heart grow fonder', en ik ben blij dat we elkaar de ruimte hebben kunnen geven om onszelf te ontwikkelen. Bedankt voor al je geduld, je luisterend oor, je schouders voor troost, maar met name voor je onvoorwaardelijke liefde, je humor, je gedrevenheid en je doorzettingsvermogen. Je bent mijn wortels en mijn vleugels.

Annerieke Sierksma

Oktober 2012 
